\title{
Reproductive strategies of alpine apomictic plants under different ecological conditions
}

\author{
Dissertation to obtain the degree \\ „Doctor rerum naturalium" \\ Ph.D. Division of Mathematics and Natural Sciences \\ of the University of Goettingen \\ within the doctoral program Biology \\ of the Georg-August University School of Science (GAUSS)
}

Submitted by

Christoph Carl-Friedrich Schinkel

born in Gießen

Göttingen, 2019 



\section{Thesis Committee}

Prof. Dr. Elvira Hörandl

Department of Systematics, Biodiversity and Evolution of Plants

Albrecht-von-Haller Institute for Plant Sciences

University of Goettingen, Germany

Prof. Dr. Stefan Scheu

Johann-Friedrich-Blumenbach Institute of Zoology and Anthropology

University of Goettingen, Germany

\section{Members of the Examination Board}

$1^{\text {st }}$ Reviewer Prof. Dr. Elvira Hörandl

Department of Systematics, Biodiversity and Evolution of Plants

Albrecht-von-Haller Institute for Plant Sciences

University of Goettingen, Germany

$2^{\text {nd }}$ Reviewer Prof. Dr. Stefan Scheu

Johann-Friedrich-Blumenbach Institute of Zoology and Anthropology

University of Goettingen, Germany

\section{Further members of the Examination Board}

Prof. Dr. Christiane Gatz

Department of Plant Molecular Biology and Physiology

Schwann-Schleiden-Research-Center for Molecular Cellbiology

University of Goettingen, Germany

PD Dr. Franz Hadacek

Department of Plant Biochemistry

University of Goettingen, Germany

Prof. Dr. Holger Kreft

Department of Biodiversity, Macroecology \& Biogeography

Faculty of Forest Sciences and Forest Ecology

University of Goettingen, Germany

Prof. Dr. Mark Maraun

Johann-Friedrich-Blumenbach Institute of Zoology and Anthropology

University of Goettingen, Germany 



\section{Acknowledgements}

First of all, I would like to thank Prof. Dr. Elvira Hörandl for the trust she placed in me to perform this research, her help and advice throughout the project and the expert support of my work, as well as Prof. Dr. Stefan Scheu for taking over the second review of my dissertation. I further thank Simone Klatt and Eleni Syngelaki for their invaluable encouragement and the countless discussions that improved the quality of my work.

Field exploration and sampling would have been impossible without the support from Bernhard Kirchheimer, Stefan Dullinger, Manuela Winkler, Agnes Dellinger, Siegrun Ertl, Christian Gilli, Franz Hadacek, Karl Hülber, and I thank Sabine Schmidt and Silvia Friedrichs for taking affectionate care of the plants in the garden.

The work was largely supported by the German Research Foundation DFG (Deutsche Forschungsgemeinschaft) [Grant number HO 4395/1-1] and the Austrian Science Fund FWF (Fonds zur Förderung der wissenschaftlichen Forschung) [Grant number I 1189].

Special thanks to Prof. Danny Geelen and Dr. Nico de Storme for allowing me to use their laboratory facilities at the Faculty of Bioscience Engineering, University of Gent, and providing me with insightful thoughts.

Finally, I would like to thank all the others, who have helped me in many ways to complete this work. 



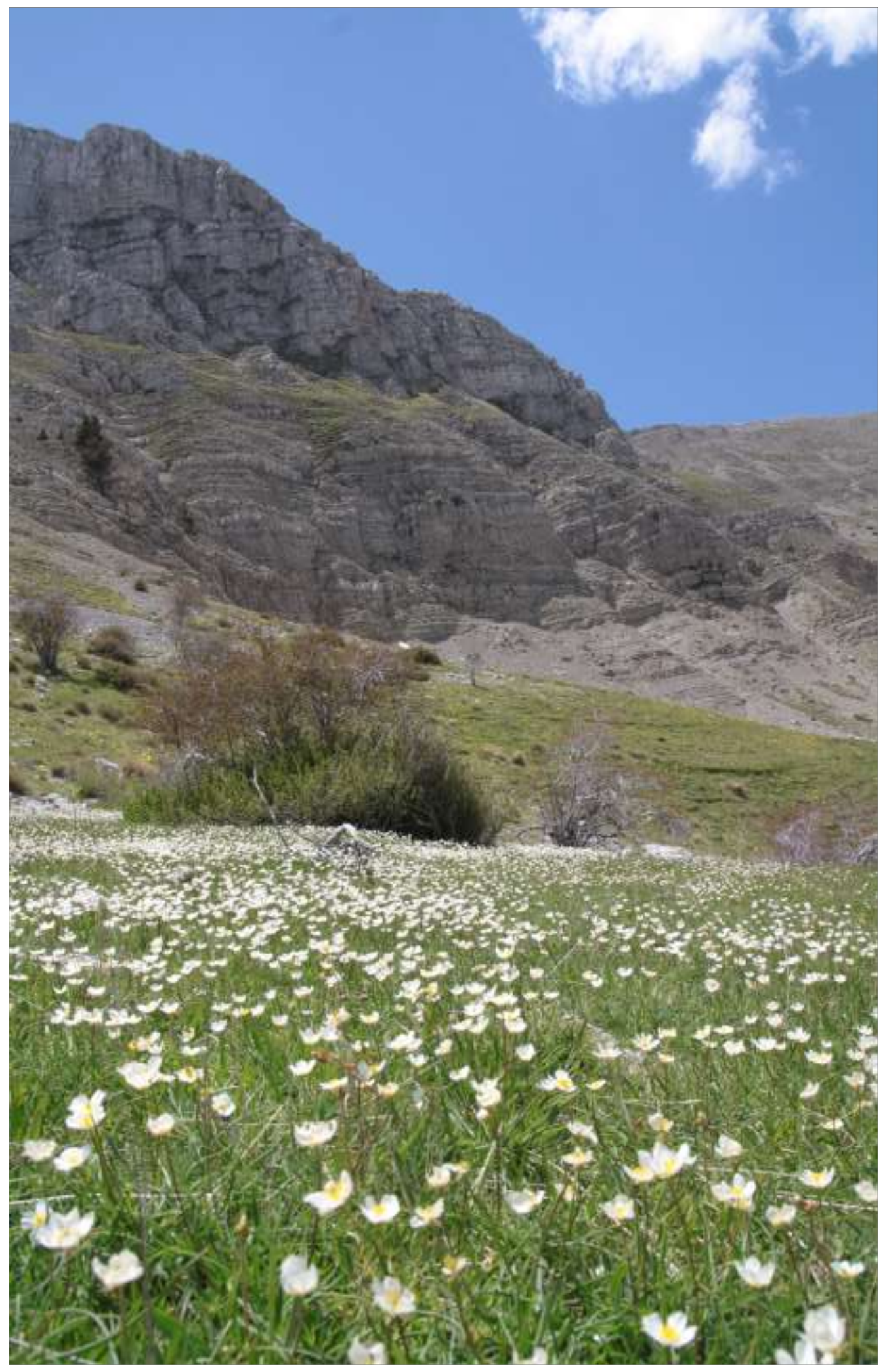

A population of diploid Ranunculus kuepferi in their natural habitat on a plateau in the French Alps. Picture taken by Christoph C. F. Schinkel. 



\section{Table of Contents}

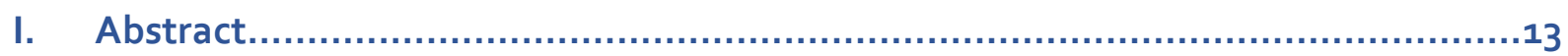

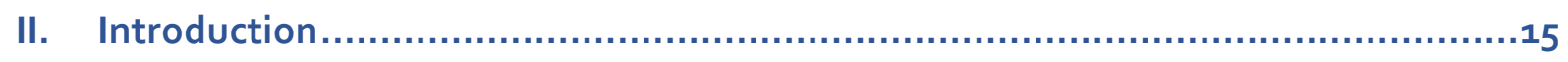

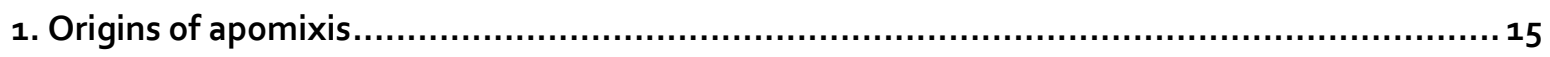

2. Geographical Parthenogenesis and possible explanations ...........................................16

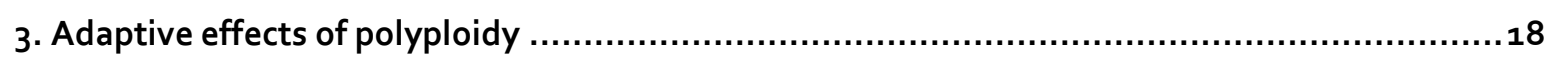

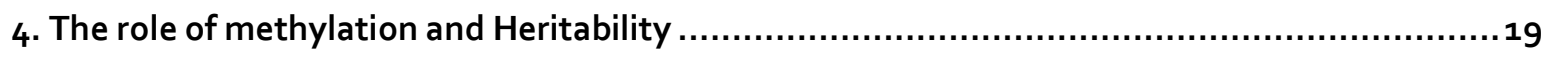

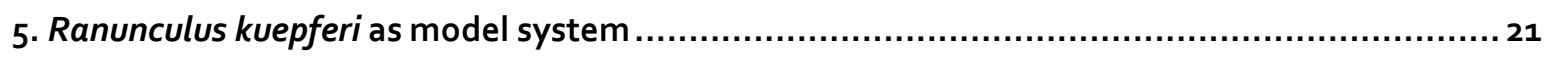

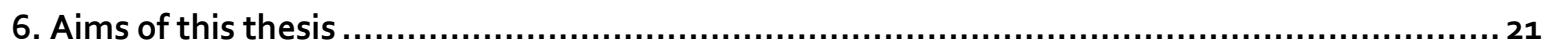

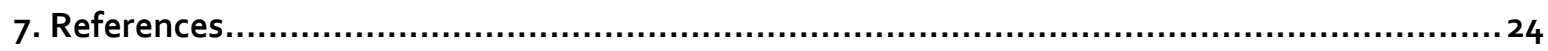

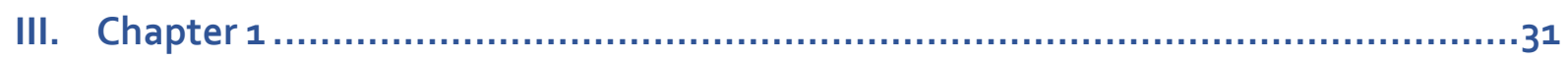

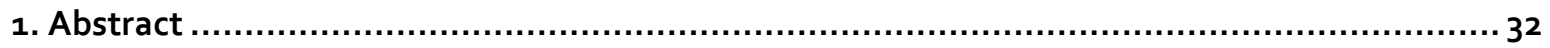

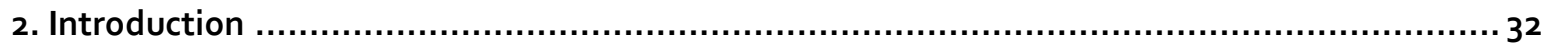

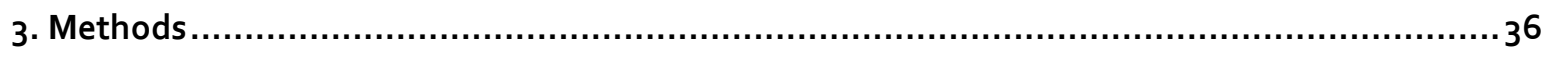

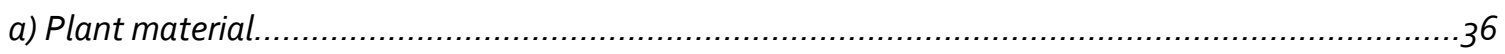

b) Flow cytometric seed screen (FCSS) and ploidy determination .............................................

c) Seed set and morphological fitness parameters ................................................................. 41

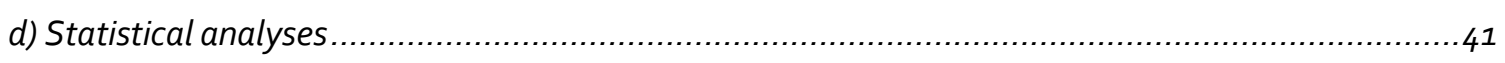

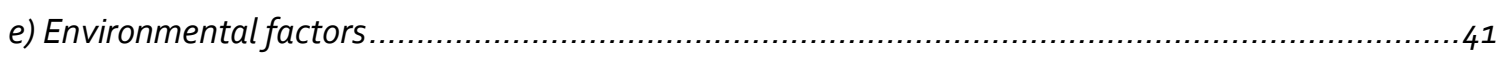

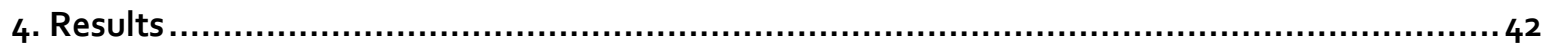

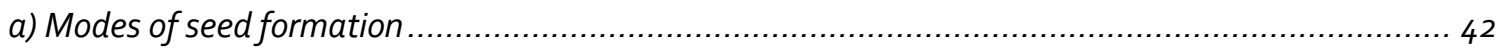

b) Variance and geographical pattern of reproduction mode of populations ..................................... 44

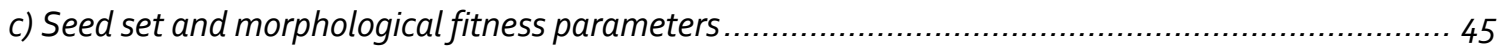

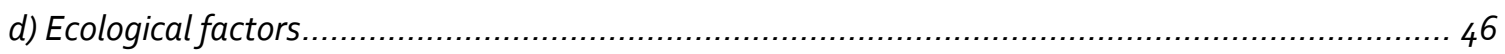

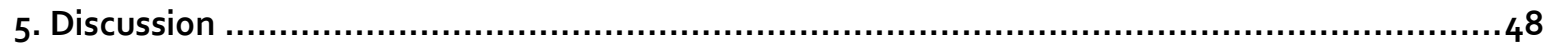

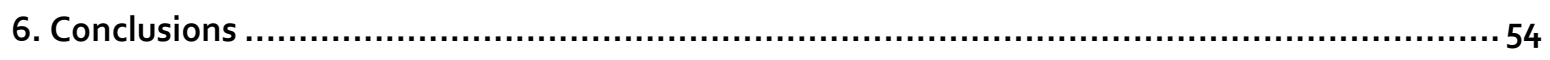

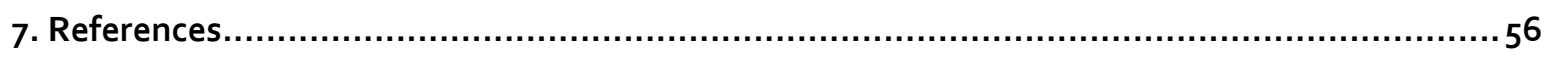

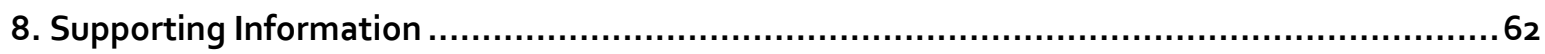




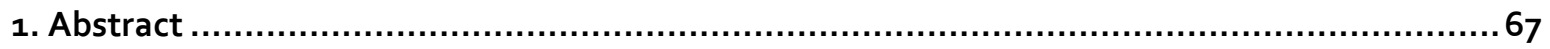

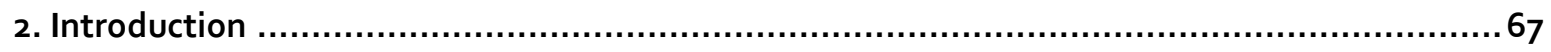

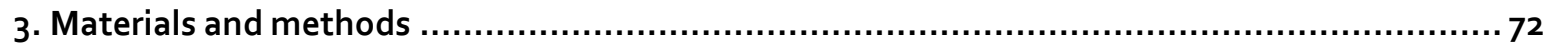

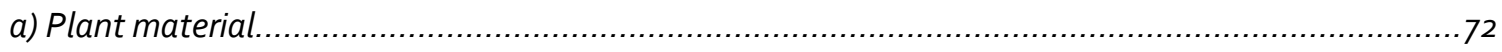

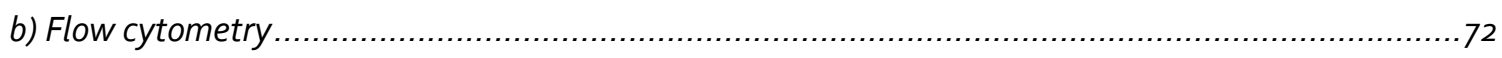

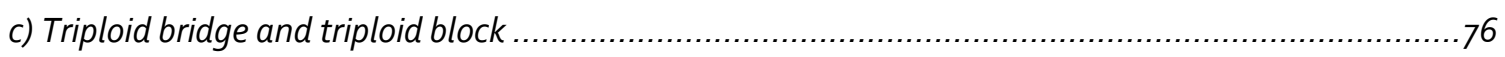

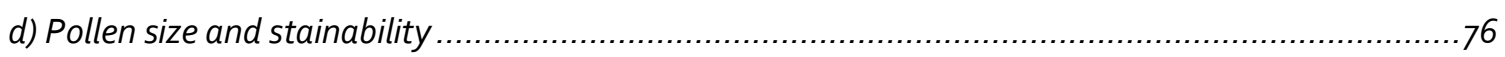

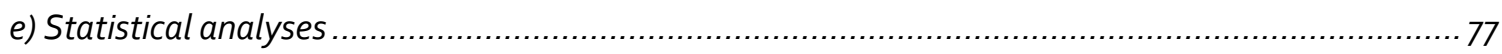

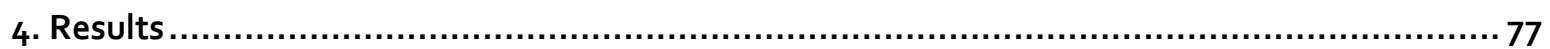

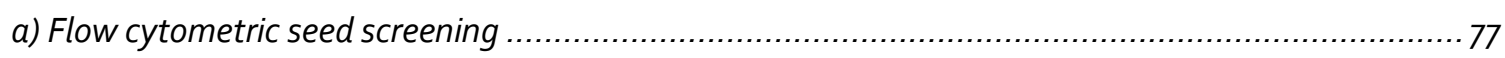

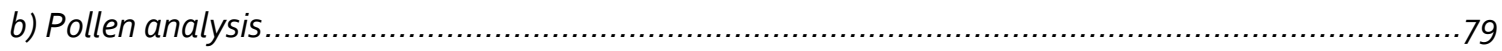

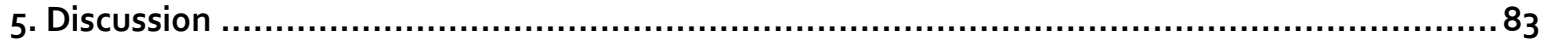

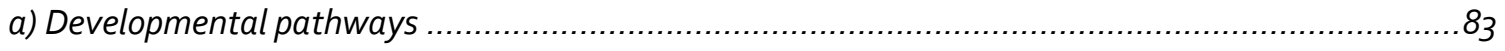

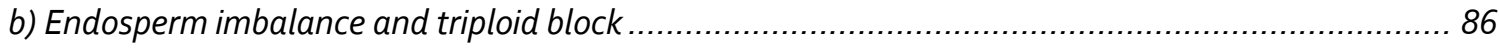

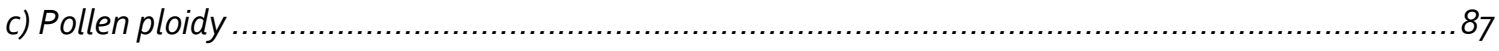

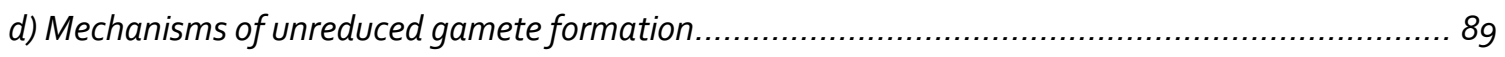

e) Implications for the evolution of polyploidy and apomixis .................................................... 89

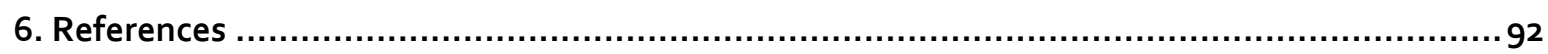

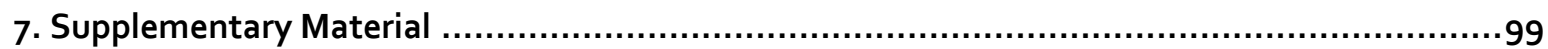

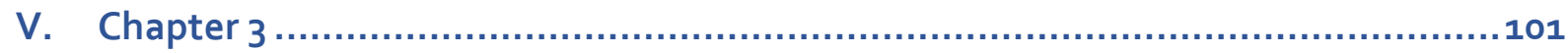

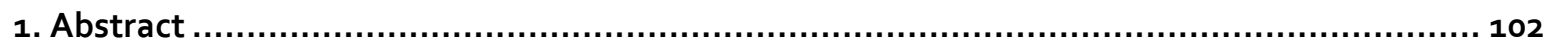

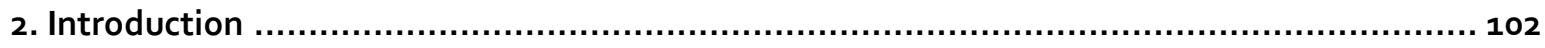

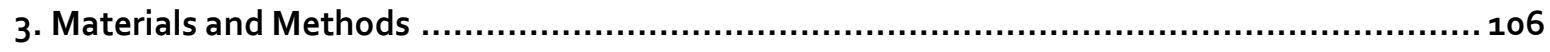

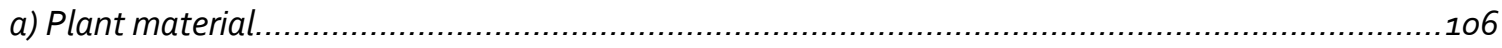

b) Methylation-Sensitive Amplified Fragment-Length Polymorphisms......................................... 107

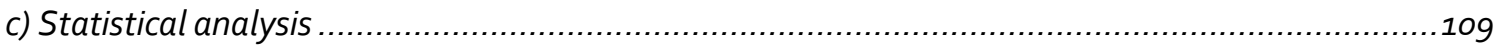

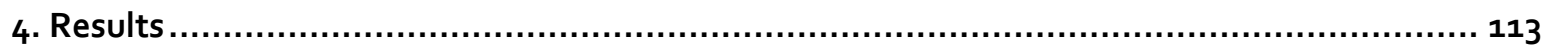

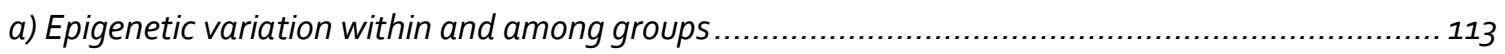

b) Correlations of MSAPs to ploidy level versus reproduction mode ............................................. 117

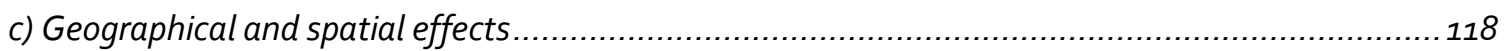

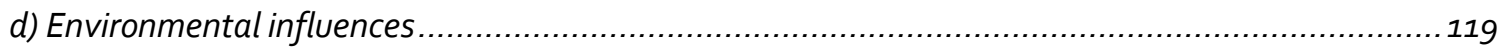


5. Discussion

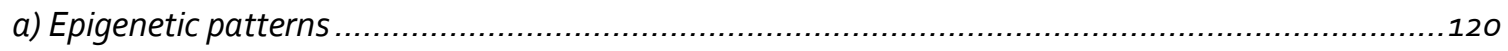

b) Geographical patterns and environmental correlations .............................................124

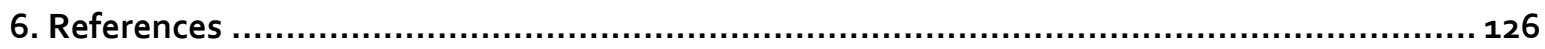

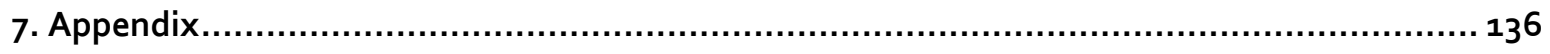

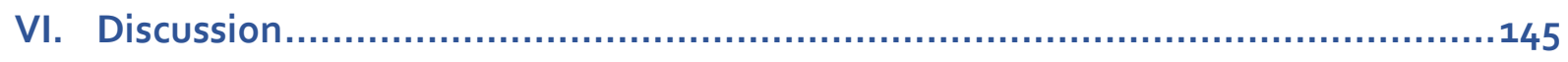

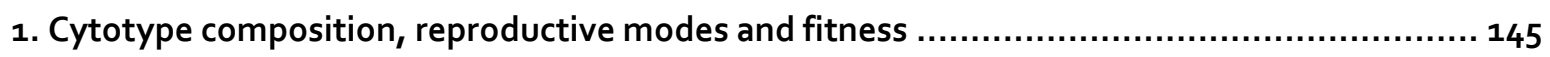

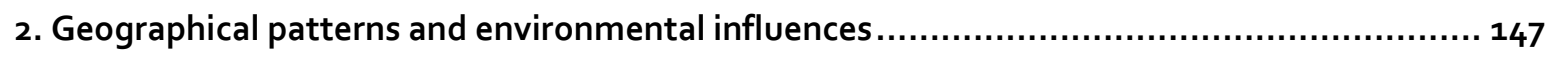

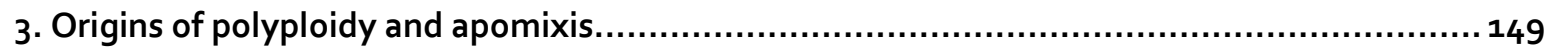

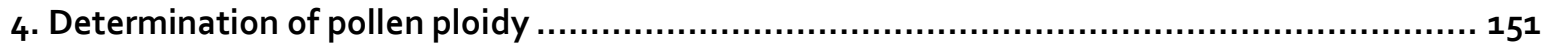

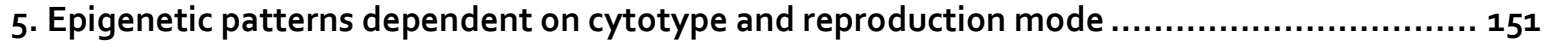

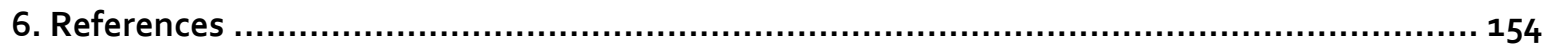

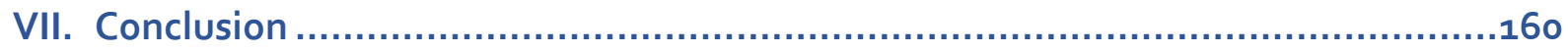





\section{Abstract}

The origin of apomixis in natural populations is still not well understood. Apomictic plants tend to expand their distribution range more to higher latitudes and altitudes compared to their sexual progenitors, a phenomenon known as Geographical Parthenogenesis. Apomixis provides an increased colonizing ability due to uniparental reproduction, as no mating partners and pollinators are needed (Baker's Law), but whether Geographical Parthenogenesis is directly related to the reproduction itself or rather a consequence of polyploidization was so far unknown. Polyploidy is one of the most important evolutionary processes in plants and thought to infer fitness advantages (higher vigor) that would enable plants to adjust better to more extreme climatic conditions. Indeed, occurrences of apomixis are often associated with colder climates. It is hypothesized that low temperatures have a direct effect on the origin of apomixis by triggering unreduced egg cell formation. Although it is assumed that apomixis is strictly connected to polyploidy, it has been shown that apomixis can also appear in diploids. This implies the possibility for polyploidization process via partial apomixis ( $B_{\text {III }}$ hybrids). Polyploids usually emerge from unreduced gametes which either fuse with reduced ones, resulting in triploid offspring (triploid bridge), or with other unreduced gametes, resulting in tetraploid embryos. Both pathways are largely unexplored regarding their frequencies and male versus female gamete contributions.

In this thesis I focused on the complex causalities of Geographical Parthenogenesis in the alpine diploid-autotetraploid system Ranunculus kuepferi among natural populations in the Alps. I conducted studies on the variation and distribution of reproductive modes, environmental influences and the genetic/epigenetic background, trying to disentangle some of the various syndetic issues in connection with polyploidy and apomixis, so that the presented outcome may contribute to a broader understanding of the dynamics and reasons behind this enigmatic phenomenon of evolution.

Results showed that apomixis emerges spontaneously among random diploid individuals of R. kuepferi in geographically isolated populations. Although frequencies of apomixis in diploids are proportionally low, unreduced female gamete formation can lead to polyploidization if subsequent fertilization takes place. Resulting $B_{\text {III }}$ hybrids were discovered in surprisingly large numbers and consisted of mostly triploid individuals. Absence of both, triploids arising from unreduced pollen and obligate sexual tetraploids, suggest a female triploid bridge as major pathway of polyploidization in $R$. kuepferi. Only one seed originated from 
biparental polyploidization. But sexual neopolyploids that develop in surrounding diploid populations are prone to fertilization with haploid pollen that would lower reproductive fitness due to endosperm imbalances and cause Minority Cytotype Exclusion. Coupling of unreduced egg cell formation (apomeiosis) to parthenogenesis result in fully functional apomixis, which reliefs disadvantageous influence of putative $F_{1}$ sterility and aids the establishment of polyploid complexes. Autopolyploidy resulting from whole genome duplication probably helped to establish respective complexes in colder climates, due to an increased physiological tolerance by adaptive features like dwarf growth. This would also explain significant ecological niche shifts towards lower temperatures among tetraploid populations. Additional low genetic divergence between di- and tetraploids indicate the involvement of epigenetic control mechanisms, which are known to play a crucial role in the response of plants to environmental conditions. Cytosine-methylation is heritable and supposedly has an important transgenerational effect on both spontaneous shifts in the mode of reproduction among same genotypes, as well as for rapid adaptations to environmental conditions. Apomixis may supported polyploid establishment and extension of occurrences to higher altitudes. Subsequent changes in the methylation pattern caused by environmental cold shocks then led to a separation between facultative and obligate tetraploid apomicts of $R$. kuepferi. 


\section{Introduction}

The study of evolutionary characteristics in apomictic plants represents one of the greatest challenges of evolutionary biology. Apomixis in the sense of agamospermy is an asexual form of reproduction among plants with clonally formed seeds (Nogler 1984, Asker and Jerling 1992, Koltunow and Grossniklaus 2003). It is widespread and found in at least 78 families and more than 290 genera of angiosperms (Hojsgaard et al. 2014a). Although the incidence among plant families is scattered across lineages, the considerable success of apomicts is of great interest in ecological and evolutionary studies (Kearny 2005). A high abundance, diversity and the geographical dispersion of some groups question the general advantage of sexvality (Vrijenhoek 1984, 1994, Van Dijk 2003, Haag and Ebert 2004, Hörandl 2009). However, processes underlying the origin of apomixis in natural populations are still not fully understood.

\section{Origins of apomixis}

Apomixis essentially consists of the two main components apomeiosis, which describes the development of unreduced female gametophytes and parthenogenesis, i.e. the independent development of unreduced oocytes. Both steps are under different genetic or respectively epigenetic control (Nogler 1984, Ozias-Akins and Van Dijk 2007). It is assumed that apomeiosis is facilitated by a spatial or temporal genomic deregulation, which destabilizes the control of sexual meiotic signaling pathways (Carman 1997, Koltunow and Grossniklaus 2003, Bicknell and Koltunow 2004, Sharbel et al. 2010, Grimanelli 2012). In the majority of cases meiosis is thus not completely switched off, but during female sporogenesis either two unreduced megaspores are formed by restitutional meiosis of which only one develops into a functional unreduced megaspore (diplospory), or female meiosis is bypassed, and an unreduced embryo sac develops from a somatic nucellus cell in parallel (apospory). Meiotic restitution itself is caused by a non-reductional cell division during meiosis resulting in dyads instead of tetrads, which contain megaspores with a somatic chromosome set. It has been recognized in numerous plant taxa and seems to be the predominant mechanism of apomeiosis (Bretagnolle and Thompson 1995, Bicknell and Koltunow 2004, Pecrix et al. 2011, De Storme and Geelen 2013, Mirzaghaderi and Hörandl 2016). Grimanelli (2012) found that an imbalanced expression of AGO protein family genes, which influence cell fate in the reproductive 
tissues, may provoke either di- or apospory. However, exact genetic components driving apomictic seed development and interactions of environmental influences are not yet fully understood.

As most apomicts are also polyploid and/or of hybridigenous origin, it is thought that the profound genomic changes and rearrangements following polyploidization/hybridization may trigger the emergence of apomixis (Carman 1997, Koltunow and Grossniklaus 2003). Polyploidy is a naturally occurring and frequent condition and as that an evident and important force of evolution (Masterson 1994, Ramsey and Schemske 1998, Soltis and Soltis 2000, Wendel 2000, Wood et al. 2009). Fossil records indicate that at least $70 \%$ of angiosperms are of polyploid origin. Some recent genomic analyses suggest even higher proportions up to the conclusion of some authors, that all angiosperms are paleopolyploids (Blanc et al. 2003, Jiao et al. 2011). Thus, polyploidy alone cannot explain the occurrence of apomixis.

Indeed, several recent studies showed apomixis to arise spontaneously among diploids. For instance, apomictic reproduction is described among diploid hybrids in the genus Boechera (Kantama et al. 2007, Aliyuh et al. 2010). Sporadic asexual seed formation is also found in natural populations of diploid Paspalum species (Siena et al. 2008) and Ranunculus kuepferi (Schinkel et al. 2016, 2017). In some cases apospory could be detected but without coupling to parthenogenesis and therefore no seed formation (Noyes 2007, Hojsgaard et al. 2014b). The existence of diploid hybrids, which reproduce mainly sexually, thus excludes also hybridization as the sole cause of apomixis. Additional genetic or non-genetic factors must be involved. Van Dijk and Vijverberg (2005) described three deleterious genetic mutations needed to acquire fully functional apomixis. Hence, spontaneous emergence of apomixis in natural populations based on a genetic mutation model seems to be unlikely.

\section{Geographical Parthenogenesis and possible explanations}

Divergent distribution patterns between apomictic and sexual taxa have been described for a long time (Vandel 1928). This phenomenon is called Geographical Parthenogenesis and discovered in animals as well as plants (Bell 1982, Bierzychudek 1985, Van Dijk 2003, Haag and Ebert 2004, Kearny 2005, Hörandl 2006, 2009, 2011, Hörandl et al. 2008, Morgan-Richards et al. 2010). For angiosperms, Bierzychudek (1985) has specified that agamic complexes usually exhibit larger areas of distribution, which are spread to both higher latitudes and altitudes, as 
well as their tendency to colonize previously glaciated areas. This characteristic is usually associated with the colonization of such habitat after the initial emergence of apomixis in natural populations (Kearny 2005, Hörandl 2006) and most probably related to the climatic fluctuations during the Pleistocene (Carman 1997, Dobeš et al. 2004, Paun et al. 2006). The Alpine zones are characterized by extreme environmental conditions like colder climates and shorter vegetation periods (Körner 2003, 2006). Cold temperatures are known to cause cold stress in plants and are known to cause unreduced gamete formation (Gustafsson and Nygren 1946, Ramsey and Schemske 1998, De Storme and Geelen 2013, Bomblies et al. 2015). A delay or acceleration of protein activity caused by cold stress can have various effects on meiosis, influencing homologous chromosome pairing, correct segregation or causes a disturbed spindle formation during meiosis I and II (De Storme and Geelen 2013b, De Storme and Mason 2014, Bomblies et al. 2015). This ultimately leads either to a complete failure of meiosis, a destabilized or unbalanced meiosis, or provokes restitutional meiosis, which in turn produces unreduced gametes. This is especially prominent in male gamete formation and unreduced pollen thereby even possess importance for sexual reproduction processes, as it is a major pathway for sexual polyploidization (Ramsey and Schemske 1998). On the other hand, alterations during the female development is much less understood, although it is the prerequisite of apomictic seed formation.

Similar to selfing plants, apomicts inherently possess the ability of founding new populations via a single propagule (Baker 1967, Baker and Stebbins 1965, Haag and Ebert 2004, Hörandl et al. 2008). In contrast to their mostly self-incompatible sexual relatives, they are able to form endosperm either pollen-independent (autogamy) or at least by self-fertilization (pseudogamy) and consequently do not necessarily require any pollinators or even mating partners (Asker and Jerling 1992, Hörandl 2009, 2010). Such uniparental reproduction is tremendously beneficial for colonization, especially after long-distance dispersal as it is described by Baker (Baker 1967, Baker and Stebbins 1965). In general, it is assumed that apomicts are able to occupy new and suitable habitats much faster than sexuals. A possible Allee effect, which plays a crucial role in colonization processes, is only of minor importance, since mate limitation is circumvented by asexual reproduction. Thereby, emergence of genetic bottlenecks after such founding events is largely mitigated by a general preservation of heterozygous genotypes by apomicts. However, Baker's law does not provide a theoretical back- 
ground why apomicts colonize higher elevations and latitudes. On the other hand, their appearance is scarce in extreme heights and northernmost latitudes, despite the large availability of open pioneer habitats, such as glacial moraine (Asker and Jerling 1992, Hörandl et al. 2011). Therefore, a better colonization performance is not sufficient as sole explanation for the distribution patterns that occur in the context of Geographical Parthenogenesis, and there must be other factors that are responsible for the success of asexually propagating organisms.

Maybe apomixis provides fundamental advantages for niche exploitation. According to the General-Purpose Genotype (GPG) model, heterogenous environments would select for those genotypes that express a high plasticity. Single successful clones from the range of available genotypes within the sexual predecessor populations would then cause the observed patterns of Geographical Parthenogenesis, as they occupy the various ecological niches available. Contradictory, such widespread general genotypes are absent in spatial distribution patterns among most studied apomictic plants (Hörandl and Paun 2007, Burnier et al. 2009, Cosendai et al. 2011). In contrast, the Frozen Niche Variation (FNV) model predicts that apomictic populations are able to exploit niche ranges as effectively as sexual populations by forming discrete arrays of clones originating from diverse genetic compositions of their progenitors (Vrijenhoek 1984, 1994, Vrijenhoek and Parker 2009). The recurrent emergence of asexual cytotypes from the same reservoir of parental genotypes would then generate a flock of different ecotypes that are protected against erosion by gene flow. Furthermore, apomictic reproduction could also fixate specialized genotypes of the progenitor population that lay outside the ecological optimum, which allow for exploitation of extreme ranges of niches. The ability to occupy such different ecological niches could provide a selection advantage for asexual organisms: a high diversity of specialized genotypes would allow a better use of total resources.

\section{Adaptive effects of polyploidy}

Polyploidy can provide more physiological and ecological flexibility along with a higher allelic diversity and heterozygosity, especially in autopolyploids (Prince and Pickett 2002, Comai 2005, Adams and Wendel 2005, Moore and Purugganan 2005, Te Beest et al. 2011). This is referred to as heterosis effects and convey some profound evolutionary advantages that may promote a better adaptation to new and harsh environments. In alpine habitats plants usually 
decrease their vegetative growth and height, which is referred to as alpine dwarfism (Körner 2003). This represents an adaptation to the shorter vegetation periods and protects against both freezing and overheating (Larcher et al. 2008). The small growth is due to a reduction in the number of cells, whereby the cell size normally remains unchanged. However, in the case of polyploids it has been shown that the cell size increases (Te Beest et al. 2011). Polyploids in cold regions thus require fewer cells for the formation of their tissues (Körner 2003, 2006, Ladinig et al. 2013) and therefore even less expenditure for their dwarf growth. Faster growth can in turn be beneficial in utilizing the low-competitive conditions immediately after snowmelt in order to establish and multiply. Smaller plants also have the advantage of better protection of their reproductive tissues at freezing temperatures that occur regularly during summer time in higher latitudes and elevations (Ladinig et al. 2013). As freezing tolerance varies among different plant species, a reduced vegetative growth could help polyploids without special adaptations to cold-shocks to better adjust during upward migration in alpine zones. Indeed, to explain the rapid range expansions that are commonly observed in many polyploids (e.g. Bierzychudek 1985, Cosendai and Hörandl 2010, Vrijenhoek and Parker 2009), a series of other phenotypic and physiological changes are of importance that affect the plasticity of traits. On the other hand, polyploids have to overcome several adverse effects before they are able to form viable populations and persist. Such side effects include strong post-zygotic barriers that cause reproductive isolation (Ramsey and Schemske 1998, Köhler et al. 2010). But also pre-zygotic barriers are known to arise like selfing, phenological separation, changes in flowering time as well as ecological differentiations like shifting optima and expanding niches breadths (Levin 1975, Segraves et al. 1999, Husband and Schemske 2000, Levin 2004).

\section{The role of methylation and Heritability}

Besides apomeiotic formation of unreduced embryo sacs, parthenogenetic development of the egg cells is a key step to fully functional gametophytic apomixis. Parthenogenesis is independently controlled by genetic or epigenetic factors (Ozias-Akins and Van Dijk 2007, Podio et al. 2014). Epigenetic chemical modifications provide advanced molecular information beyond the measure of the genetic sequence and can be under constant transformation throughout the lifetime of an organism. Although there is no alteration of the under- 
lying DNA sequences, epigenetic modifications are able to interact on phenotypic characteristics (Richards 2006, Bird 2007, Verhoeven et al. 2016). The methylation of $5^{\prime}$-cytosine is considered one of the most important epigenetic mechanisms (Jaenisch and Bird 2003, Bender 2004). It affects individual development by enhanced control of gene regulation. The activity of certain genomic segments can be activated or reduced respectively disabled, which can be triggered by biotic and abiotic stimuli causing de novo and demethylation processes and resulting in different DNA methylation patterns (e.g. Labra et al 2002, Sherman and Talbert 2002, Grant-Downton and Dickinson 2005, Berger 2007, Dowen et al. 2012, Matzke et al. 2015, Espinas et al. 2016). This can have profound effects on ecologically relevant traits and plasticity by imprinting of heterochromatic loci, as well as the silencing of both transposons and transgenes (Miura et al. 2001, Grossniklaus et al. 2001, Lippman et al. 2004, Zilberman et al. 2007, Jones 2012).

It is still unclear today whether apomixis in diploids is a heritable trait or whether the hereditability among polyploids is genetically or epigenetically determined. As apomixis bypasses meiotic resetting of methylation, favorable methylation patterns could be retained over generations. Methylation profiles induced by cold stress would persist, regardless of current climatic conditions. It is further known that genomic rearrangement due to hybridization or polyploidization causes stress that in turn may induces methylation changes (Adams and Wendel 2005, Dong et al. 2006, Grant-Downton and Dickinson 2005). Such epigenetic variation sometimes produces more selectable and heritable variation than genomic mixing and possibly promotes rapid adaptation to stressful environments (Herrera and Bazaga 2013, Herman and Sultan 2016, Banta and Richards 2018, Ecker et al. 2018). In this case polyploidy may not be the primary factor for the emergence of apomixis, but rather the cause for its establishment. 


\section{Ranunculus kuepferi as model system}

An ideal model system to study the above-mentioned phenomena resembles the alpine plant Ranunculus kuepferi Greuter \& Burdet (Ranunculaceae). The species is native to the European Alps and consists of several known cytotypes, which also differ in their reproduction modes. Sexual diploids retained their refugial area in the South-western Alps, while tetraploids are presumably of multiple origin and colonized the total range of the species (Burnier et al. 2009, Cosendai et al. 2011). Other ploidy levels like tri- or hexaploids are infrequent and only occur in the sympatric contact zone of di- and tetraploids. Cosendai et al. $(2011,2013)$ proposes an autopolyploid emergence of tetraploids, since genotypic diversity and levels of heterozygosity are equal compared to diploids. Previous investigations on the mode of reproduction suggests that apomixis is predominant among tetraploids and occurs in the whole distribution range (Cosendai and Hörandl 2010). Asexual embryo sac formation in $R$. kuepferi starts from somatic cells of the nucellus, which is known as apospory (Burnier et al. 2009). Often sexual i.e. meiotic development takes place in parallel. Both processes then compete directly, with only one of the two pathways accomplishing per ovule. Bagging experiments confirmed self-fertility in tetraploids, while diploids are largely self-incompatible (Cosendai et al. 2013). So far it is unknown to which extent such facultative expression of apomixis is distributed among tetraploid populations. However, tetraploid apomicts exhibit highly facultative apospory, with indirect evidence for irregular meiosis (Burnier et al. 2009, Cosendai et al. 2011). Influences of ecological factors like climatic conditions on the distribution patterns and the mode of reproduction are rather unknown. Di- and tetraploid population of $R$. kuepferi show a divergence in their altitudinal occurrence. That is also reflected regarding the mode of reproduction, with sexuals occurring from 1300 to $2500 \mathrm{~m}$ on average, while apomicts are rather found at slightly higher altitudes from 1700 to $2800 \mathrm{~m}$ (Cosendai and Hörandl 2010, Schinkel et al. 2016).

\section{Aims of this thesis}

This thesis aims to shed light on some unresolved questions of evolutionary biology in plants. Based on the alpine model system Ranunculus kuepferi the challenging phenomenon of Geographical Parthenogenesis was examined to test the validity of competing hypothesis regarding influences of apomixis, polyploidy and epigenetics, or how they themselves are interconnected and influence each other. Multidisciplinary approaches were applied with 
methods from karyology, ecology and genetics to evaluate phenotypic plasticity of reproduction modes, and how this relates to the vegetative and reproductive fitness of apomicts versus their sexual relatives. It was also tested how large the colonization potential of apomicts is, which pathways in the development of polyploidy occur most frequently, or how these are related to pollen and possibly disturbed male gametogenesis, and the role of epigenetic methylation patterns in all that.

Chapter 1 presents a comprehensive analysis of reproductive pathways and correlated fitness parameters of di- and polyploid $R$. kuepferi samples from 81 populations across the entire distribution area in the European Alps. The employed Flow Cytometric Seed Screening method according to Doležel et al. (2007) has shown to be highly effective in the detection of different developmental pathways of the analyzed seeds. It was examined if the mode of reproduction is strictly correlated to ploidy and whether apomixis or sexual polyploidy is more successful in observed range expansions. A general hypothesis is that apomixis might be triggered by cold stress (Ramsey and Schemske 1998). Therefore, I also addressed the question if geographic distance or rather elevation and associated environmental factors such as temperature and precipitation are the most decisive factors regarding the proportions of apomictic reproduction, by analyzing putative correlations. Additionally, measurements of seed set and morphological parameters provided quantitative insights to possible differences in reproductive and trait fitness between cytotypes and reproduction modes, and also have been correlated to altitude and environmental factors.

Chapter 2 focusses on possible modes of polyploidization in $R$. kuepferi and implications for the evolution of polyploidy as well as apomixis. Many observations of triploid samples from the sympatric contact zone between di- and tetraploids found in the FCSS dataset (Chapter 1), lead to the investigation of $B_{\| I}$ hybrids that result from the fertilization of unreduced egg cells. I investigated whether both occurrences of triploids and partial apomixis represent possible examples of a unilateral triploid bridge for polyploidization or result from multiple secondary backcrossing events of established tetraploids with diploids. Possible pathways of formation among seeds that exhibited ploidy shifts compared to their di-, tri- and tetraploid mother plants were analyzed. Results were evaluated regarding endosperm contributions to 
discriminate between female versus male polyploidization and resulting endosperm imbalances, that maybe act as triploid block. Furthermore, pollen size was examined with a volumetric high-throughput method to test for a supposed correlation to pollen ploidy, indicating of unreduced male gametogenesis and consequently incidence of bilateral polyploidization.

Chapter 3 deals with epigenetic modifications in $R$. kuepferi and their variation between diand tetraploids, as potential explanation of observed shifts in the reproduction mode and ecological niches, due to the low genetic divergence between cytotypes (Cosendai et al. 2013). Cytosine-methylation was examined via Methylation-Sensitive Amplified FragmentLength Polymorphisms (MSAP) to gain better insights into correlations of epigenetic patterns to the expression of apomixis between cytotypes. The MSAP method have been recognized as informative tool for analyzing rapid adaptation in plants (Verhoeven et al. 2010, Paun et al. 2010). In addition, correlations of methylation patterns with climatic variables that proved to be important (see Chapter 1 and Kirchheimer et al. 2016, 2018) were calculated to obtain a better understanding of the adaptive potential of epigenetic mechanisms and its role in the explanation of Geographical Parthenogenesis.

By means of the accomplished extensive research, this thesis contributes significantly to a broader understanding of environmentally driven evolution in high-mountain systems. 


\section{References}

Adams KL and Wendel JF. 2005. Polyploidy and genome evolution in plants. Current Opinion in Plant Biology 8:135-141

Aliyu OM, Schranz ME and Sharbel TF. 2010. Quantitative variation for apomictic reproduction in the genus Boechera (Brassicaceae). American Journal of Botany 97:1719-1731

Asker SE and Jerling L. 1992. Apomixis in Plants. CRC Press, Boca Raton

Baker HG. 1967. Support for Baker's Law-as a rule. Evolution 21:853-856

Baker HG and Stebbins GL. 1965. Genetics of colonizing species: Proceedings of the first International Union of Biological Sciences Symposia on General Biology. Academic Press, New York

Banta JA and Richards CL. 2018. Quantitative epigenetics and evolution. Heredity 121:210-224

Bell G. 1982. The Masterpiece of Nature: The Evolution and Genetics of Sexuality. Cambridge University Press Archive, Cambridge

Bender J. 2004. DNA methylation and epigenetics. Annual Review of Plant Biology 55:41-68

Berger SL. 2007. The complex language of chromatin regulation during transcription. Nature 447:407-412

Bicknell RA and Koltunow AM. 2004. Understanding Apomixis: Recent Advances and Remaining Conundrums. The Plant Cell 16:228-245

Bierzychudek P. 1985. Patterns in plant parthenogenesis. Experientia 41:1255-1264

Bird A. 2007. Perceptions of epigenetics. Nature 447:396-398

Blanc G, Hokamp K and Wolfe KH. 2003. A recent polyploidy superimposed on older large-scale duplications in the Arabidopsis genome. Genome Research 13:137-144

Bomblies K, Higgins JD and Yant L. 2015. Meiosis evolves: adaptation to external and internal environments. New Phytologist 208:306-323

Bretagnolle F and Thompson JD. 1995. Gametes with the somatic chromosome number: mechanisms of their formation and role in the evolution of autopolyploid plants. New Phytologist 129:1-22

Burnier J, Buerki S, Arrigo N, Küpfer P and Alvarez N. 2009. Genetic structure and evolution of Alpine polyploid complexes: Ranunculus kuepferi (Ranunculaceae) as a case study. Molecular Ecology 18:3730-3744

Carman JG. 1997. Asynchronous expression of duplicate genes in angiosperms may cause apomixis, bispory, tetraspory, and polyembryony. Biological Journal of the Linnean Society 61:51-94

Comai L. 2005. The advantages and disadvantages of being polyploid. Nature Reviews Genetics 6:836-846

Cosendai A-C and Hörandl E. 2010. Cytotype stability, facultative apomixis and Geographical Parthenogenesis in Ranunculus kuepferi (Ranunculaceae). Annals of Botany 105:457-470

Cosendai A-C, Rodewald J and Hörandl E. 2011. Origin and distribution of autopolyploids via apomixis in the alpine species Ranunculus kuepferi (Ranunculaceae). Taxon 60:355-364 
Cosendai A-C, Wagner J, Ladinig U, Rosche C and Hörandl E. 2013. Geographical parthenogenesis and population genetic structure in the alpine species Ranunculus kuepferi (Ranunculaceae). Heredity 110:560-569

De Storme N and Geelen D. 2013. Sexual Polyploidization in Plants: Cytological Mechanisms and Molecular Regulation. New Phytologist 198:670-684

De Storme N and Geelen D. 2013. Cytokinesis in plant male meiosis. Plant Signaling \& Behavior 8(3):e23394

De Storme N and Mason A. 2014. Plant speciation through chromosome instability and ploidy change: cellular mechanisms, molecular factors and evolutionary relevance. Current Plant Biology 1:10-33

Dobeš C, Mitchell-Olds T and Koch M. 2004. Extensive chloroplast haplotype variation indicates Pleistocene hybridization and radiation of North American Arabis drummondii, A. x divaricarpa, and $A$. holboellii (Brassicaceae). Molecular Ecology 13:349-370

Doležel J, Greilhuber J and Suda J. 2007. Estimation of nuclear DNA content in plants using flow cytometry. Nature Protocols 2:2233-2244

Dong ZY, Wang YM, Zhang ZJ, Shen Y, Lin XY, Ou XF, Han FP and Liu B. 2006. Extent and pattern of DNA methylation alteration in rice lines derived from introgressive hybridization of rice and $\mathrm{Zi}$ zania latifolia Griseb. Theoretical and Applied Genetics 113:196-205

Dowen RH, Pelizzola M, Schmitz RJ, Lister R, Dowen JM, Nery JR and Ecker JR. 2012. Widespread dynamic DNA methylation in response to biotic stress. Proceedings of the National Academy of Sciences of the United States of America. 109:e2183-e2191

Ecker S, Pancaldi V, Valencia A, Beck S and Paul DS. 2018. Epigenetic and Transcriptional Variability Shape Phenotypic Plasticity. Bioessays 40:1700148

Espinas NA, Saze H and Saijo Y. 2016. Epigenetic Control of Defense Signaling and Priming in Plants. Frontiers in Plant Science 7:1201

Grant-Downton RT and Dickinson HG. 2005. Epigenetics and its implications for plant biology: 1. The epigenetic network in plants. Annals of Botany 96:1143-1164

Grimanelli D. 2012. Epigenetic regulation of reproductive development and the emergence of apomixis in angiosperms. Current Opinion in Plant Biology 15:57-62

Grossniklaus U, Nogler GA and van Dijk PJ. 2001. How to avoid sex: the genetic control of gametophytic apomixis. The Plant Cell 13:1491-1498

Gustafsson A and Nygren A. 1946. The temperature effect on pollen formation and meiosis in Hieracium robustum. Hereditas 32(1):1-14

Haag CR and Ebert D. 2004. A new hypothesis to explain geographic parthenogenesis. Annales Zoologicae Fennicae 41:539-544

Herman JJ and Sultan SE. 2016. DNA methylation mediates genetic variation for adaptive transgenerational plasticity. Proceedings of the Royal Society B: Biological Sciences 283(1838):20160988 
Herrera CM and Bazaga P. 2013. Epigenetic correlates of plant phenotypic plasticity: DNA methylation differs between prickly and nonprickly leaves in heterophyllous Ilex aquifolium (Aquifoliaceae) trees. Botanical Journal of the Linnean Society 171:441-452

Hojsgaard D, Greilhuber J, Pellino M, Paun O, Sharbel TF and Hörandl E. 2014. Emergence of apospory and bypass of meiosis via apomixis after sexual hybridisation and polyploidisation. New Phytologist 204:1000-1012

Hojsgaard D, Klatt S, Baier R, Carman JG and Hörandl E. 2014. Taxonomy and Biogeography of Apomixis in Angiosperms and Associated Biodiversity Characteristics. Critical Reviews in Plant Sciences $33: 414-427$

Hörandl E. 2006. The complex causality of Geographical Parthenogenesis. New Phytologist 171(3):525-538

Hörandl E. 2009. Geographical parthenogenesis: opportunities for asexuality. In: Schön I, Martens K, Van Dijk P (eds) Lost Sex. Springer, Heidelberg, pp 161-186

Hörandl E. 2010. The evolution of self-fertility in apomictic plants. Sexual Plant Reproduction 23:73-86

Hörandl E. 2011. Evolution and biogeography of alpine apomictic plants. Taxon 60:390-402

Hörandl E and Paun O. 2007. Patterns and sources of genetic diversity in apomictic plants: implications for evolutionary potentials. In: Hörandl E (eds) Apomixis: Evolution, mechanisms and perspectives. Gantner, Ruggell, pp 169-194

Hörandl E, Cosendai A-C and Temsch EM. 2008. Understanding the geographic distributions of apomictic plants: a case for a pluralistic approach. Plant Ecology and Diversity 1:309-320

Hörandl E, Dobeš C, Suda J, Vit P, Urfus T, Temsch EM, Cosendai A-C, Wagner J and Ladinig U. 2011. Apomixis is not prevalent in subnival to nival plants of the European Alps. Annals of Botany 108:381-390

Husband BC and Schemske DW. 2000. Ecological mechanisms of reproductive isolation and coexistence of diploid and tetraploid Chamerion angustifolium. Journal of Ecology 88:1-14

Jaenisch R and Bird A. 2003. Epigenetic regulation of gene expression: how the genome integrates intrinsic and environmental signals. Nature Genetics 33:245-254

Jiao Y, Wickett NJ, Ayyampalayam S, Chanderbali AS, Landherr L, Ralph PE, Tomsho LP, Hu Y, Liang $H$, Soltis PS, Soltis DE, Clifton SW, Schlarbaum SE, Schuster SC, Ma H, Leebens-Mack J and de Pamphilis CW. 2011. Ancestral polyploidy in seed plants and angiosperms. Nature 473:97-100

Jones PA. 2012. Functions of DNA methylation: islands, start sites, gene bodies and beyond. Nature Reviews Genetics 13:484-492

Kantama L, Sharbel TF, Schranz ME, Mitchell-Olds T, de Vries SC and de Jong JHSGM. 2007. Diploid apomicts of the Boechera holboellii complex display large-scale chromosome substitutions and aberrant chromosomes. Proceedings of the National Academy of Sciences of the United States of America. 104:14026-14031

Kearney M. 2005. Hybridization, glaciation and Geographical Parthenogenesis. Trends in Ecology \& Evolution 20:495-502 
Kirchheimer B, Schinkel CCF, Dellinger AS, Klatt S, Moser D, Winkler M, Lenoir J, Caccianiga M, Guisan A, Nieto-Lugilde D, Svenning J-C, Thuiller W, Vittoz P, Willner W, Zimmermann NE, Hörandl E and Dullinger S. 2016. A matter of scale: apparent niche differentiation of diploid and tetraploid plants may depend on extent and grain of analysis. Journal of Biogeography 43:716-726

Kirchheimer B, Wessely J, Gattringer A, Hülber K, Moser D, Schinkel CCF, Appelhans M, Klatt S, Caccianiga M, Dellinger A, Guisan A, Kuttner M, Lenoir J, Maiorano L, Nieto-Lugilde D, Plutzar C, Svenning J-C, Willner W, HörandI E and Dullinger S. 2018. Reconstructing Geographical Parthenogenesis: effects of niche differentiation and reproductive mode on Holocene range expansion of an alpine plant. Ecology Letters 21:392-401

Köhler C, Mittelsten-Scheid O and Erilova A. 2010. The impact of the triploid block on the origin and evolution of polyploid plants. Trends in Genetics 26:142-148

Koltunow AM and Grossniklaus U. 2003. Apomixis: a developmental perspective. Annual Review of Plant Biology 54:547-574

Körner C. 2003. Alpine plant life: functional plant ecology of high mountain ecosystems; with 47 tables. Springer, Berlin

Körner C. 2006. Significance of Temperature in Plant Life. In: Morison Jl, Morecroft MD (eds) Plant Growth and Climate Change. Blackwell Publishing Ltd., Oxford, pp 48-69

Labra M, Ghiani A, Citterio S, Sgorbati S, Sala F, Vannini C, Ruffini-Castiglione M and Bracale M. 2002. Analysis of cytosine methylation pattern in response to water deficit in pea root tips. Plant Biology 4:694-699

Ladinig U, Hacker J, Neuner G and Wagner J. 2013. How endangered is sexual reproduction of highmountain plants by summer frosts? Frost resistance, frequency of frost events and risk assessment. Oecologia 171:743-760

Larcher W, Kainmüller C and Wagner J. 2008. Survival types of high mountain plants under extreme temperatures. Flora 205:3-18

Levin DA. 1975. Minority cytotype exclusion in local plant populations. Taxon 24:35-43

Levin DA. 2004. The ecological transition in speciation. New Phytologist 161:91-96

Lippman Z, Gendrel AV, Black M, Vaughn MW, Dedhia N, McCombie WR, Lavine K, Mittal V, May B, Kasschau KD, Carrington JC, Doerge RW, Colot V and Martienssen R. 2004. Role of transposable elements in heterochromatin and epigenetic control. Nature 430:471-476

Masterson J. 1994. Stomatal size in fossil plants: evidence for polyploidy in majority of angiosperms. Science 264:421-24

Matzke MA, Kanno T and Matzke AJM. 2015. RNA-Directed DNA Methylation: The Evolution of a Complex Epigenetic Pathway in Flowering Plants. Annual Review of Plant Biology 66:243-267

Mirzaghaderi G, Hörandl E. 2016. The evolution of meiotic sex and its alternatives. Proceedings of the Royal Society B: Biological Sciences 283:20161221 
Miura A, Yonebayashi S, Watanabe K, Toyama T, Shimada H and Kakutani T. 2001. Mobilization of transposons by a mutation abolishing full DNA methylation in Arabidopsis. Nature 411:212-214

Moore RC and Purugganan MD. 2005. The evolutionary dynamics of plant duplicate genes. Current Opinion in Plant Biology 8:122-128

Morgan-Richards MARY, Trewick SA and Stringer IA. 2010. Geographic parthenogenesis and the common tea-tree stick insect of New Zealand. Molecular Ecology 19(6):1227-1238

Nogler GA. 1984. Gametophytic apomixis. In: Johri PBM (eds) Embryology of angiosperms. Springer, Berlin, $p p$ 475-518

Noyes RD. 2007. Apomixis in the Asteraceae: diamonds in the rough. Functional plant science and biotechnology $1(2): 207-222$

Ozias-Akins P and Van Dijk PJ. 2007. Mendelian genetics of apomixis in plants. Annual Review of Genetics 41:509-537

Paun O, Bateman RM, Fay MF, Hedrén M, Civeyrel L and Chase MW. 2010. Stable Epigenetic Effects Impact Adaptation in Allopolyploid Orchids (Dactylorhiza: Orchidaceae). Molecular Biology and Evolution 27(11):2465-2473

Paun O, Stuetty TF and Hörandl E. 2006. The role of hybridization, polyploidization and glaciation in the origin and evolution of the apomictic Ranunculus cassubicus complex. New Phytologist 171:223236

Pecrix Y, Rallo G, Folzer H, Cigna M, Gudin S and Le Bris M. 2011. Polyploidization mechanisms: temperature environment can induce diploid gamete formation in Rosa sp. Journal of Experimental Botany 62:3587-3597

Podio M, Felitti SA, Siena LA, Delgado L, Mancini M, Seijo JG, González AM, Pessino SC and Ortiz JPA. 2014. Characterization and expression analysis of SOMATIC EMBRYOGENESIS RECEPTOR KINASE (SERK) genes in sexual and apomictic Paspalum notatum. Plant Molecular Biology 84:479495

Prince VE and Pickett FB. 2002. Splitting pairs: the diverging fates of duplicated genes. Nature Reviews Genetics 3:827-837

Ramsey J and Schemske DW. 1998. Pathways, mechanisms, and rates of polyploid formation in flowering plants. Annual Review of Ecology and Systematics 29:467-501

Richards EJ. 2006. Inherited epigenetic variation-revisiting soft inheritance. Nature Reviews 7:395-401

Schinkel CCF, Kirchheimer B, Dellinger AS, Klatt S, Winkler M, Dullinger S and Hörandl E. 2016. Correlations of polyploidy and apomixis with elevation and associated environmental gradients in an alpine plant. AoB PLANTS 8:plwo64

Schinkel CCF, Kirchheimer B, Dullinger S, Geelen D, De Storme N and Hörandl E. 2017. Pathways to polyploidy: indications of a female triploid bridge in the alpine species Ranunculus kuepferi (Ranunculaceae). Plant Systematics and Evolution 303:1093-1108 
Segraves KA, Thompson JN, Soltis PS and Soltis DE. 1999. Multiple origins of polyploidy and the geographic structure of Heuchera grossulariifolia. Molecular Ecology 8:253-262

Sharbel TF, Voigt M-L, Corral JM, Galla G, Kumlehn J, Klukas C, Schreiber F, Vogel H and Rotter B. 2010. Apomictic and Sexual Ovules of Boechera Display Heterochronic Global Gene Expression Patterns. The Plant Cell 22(3):655-671

Sherman JD and Talbert LE. 2002. Vernalization-induced changes of DNA methylation pattern in winter wheat. Genome 45:253-260

Siena LA, Sartor ME, Espinoza F, Quarin CL and Ortiz JPA. 2008. Genetic and embryological evidences of apomixis at the diploid level in Paspalum rufum support recurrent auto-polyploidization in the species. Sexual Plant Reproduction 21:205-215

Soltis DE and Soltis PS. 2000. Contributions of plant molecular systematics to studies of molecular evolution. Plant Molecular Biology Reporter 42:45-75

Te Beest M, Le Roux JJ, Richardson DM, Brysting AK, Suda J, Kubešová M and Pyšek P. 2011. The more the better? The role of polyploidy in facilitating plant invasions. Annals of Botany 109(1):19-45

Van Dijk PJ. 2003. Ecological and evolutionary opportunities of apomixis: insights from Taraxacum and Chondrilla. Philosophical Transactions of The Royal Society B: Biological Sciences 358:1113-1121

Van Dijk PJ and Vijverberg K. 2005. The significance of apomixis in the evolution of the angiosperms: a reappraisal. In: Bakker F, Chatrou L, Gravendeel B, Pelser PB (eds) Plant species-level systematics: new perspectives on pattern \& process. Gantner, Ruggell, $p p$ 101-116

Vandel A. 1928. La parthenogenese geographique: Contribution a l'etude biologique et cytologique de la parthenogenese naturelle. Bulletin biologique de la France et de la Belgique 62:164-281

Verhoeven KJF, Jansen JJ, van Dijk PJ and Biere A. 2010. Stress-induced DNA methylation changes and their heritability in asexual dandelions. New Phytologist 185:1108-18

Verhoeven KJF, von Holdt MB and Sork VL. 2016. Epigenetics in ecology and evolution: what we know and what we need to know. Molecular Ecology 25:1631-1638

Vrijenhoek RC. 1984. Ecological differentiation among clones: The Frozen Niche Variation model. In: Wöhrmann K, Loeschcke V (eds) Population Biology and Evolution. Springer, Berlin, pp 217-231

Vrijenhoek RC. 1994. Unisexual fish: Model systems for studying ecology and evolution. Annual Review of Ecology and Systematics 25:71-96

Vrijenhoek RC and Parker ED Jr. 2009. Geographical Parthenogenesis: General Purpose Genotypes and Frozen Niche Variation. In: Schön I, Martens K and Van Dijk PJ (eds) Lost Sex. Springer, Dordrecht, pp 99-131

Wendel JF. 2000. Genome evolution in polyploids. Plant Molecular Biology Reporter 42:225-249

Wood TE, Takebayashi N, Barker MS, Mayrose I, Greenspoon PB and Rieseberg LH. 2009. The frequency of polyploid speciation in vascular plants. Proceedings of the National Academy of Sciences of the United States of America. 106:13875-13879 
Zilberman D, Gehring M, Tran RK, Ballinger T and Henikoff S. 2007. Genome-wide analysis of Arabidopsis thaliana DNA methylation uncovers an independence between methylation and transcription. Nature Genetics 39:61-69 


\section{Chapter 1}

\section{Correlations of polyploidy and apomixis with elevation and associated en-} vironmental gradients in an alpine plant

Christoph C. F. Schinkel, Bernhard Kirchheimer, Agnes S. Dellinger, Simone Klatt, Manuela

Winkler, Stefan Dullinger and Elvira Hörandl

Published in AoB PLANTS (2016) 8:plwo64

DOI 10.1093/aobpla/plwo64

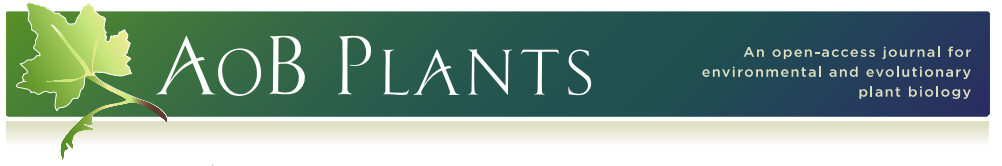

Research Article

\section{Correlations of polyploidy and apomixis with elevation and associated environmental gradients in an alpine plant}

Christoph C. F. Schinkel ${ }^{1}$, Bernhard Kirchheimer ${ }^{2}$, Agnes S. Dellinger ${ }^{2}$, Simone Klatt ${ }^{1}$, Manuela Winkler ${ }^{3}$, Stefan Dullinger ${ }^{2}$ and Elvira Hörandl ${ }^{\star 1}$

${ }^{1}$ Department of Systematics, Biodiversity and Evolution of Plants (with herbarium), Georg-August-University of Göttingen, Untere Karspule 2, 37073 Gottingen, Germany

Sesearch, University of Vienna, Rennweg 14, 1030 Vienna, Austria Vienna, Austria

Received: 13 April 2016; Accepted: 29 July 2016; Published: 4 September 2016

Associate Editor: Simon J. Hiscock

Citation: Schinkel CCF, Kirchheimer B, Dellinger AS, Klatt S, Winkler M, Dullinger S, Hörandl E. 2016. Correlations of polyploidy and plw064

Abstract. Apomictic plants expand their geographical distributions more to higher elevations compared to their sexual progenitors. It was so far unclear whether this tendency is related to mode of reproduction itself or represents a side effect of polyploidy. Apomixis is advantageous for range expansions as no mating partners and pollinators are needed (Baker's rule). Polyploidy is thought to infer fitness advantages and a higher vigour that would enable plants to adjust better to more extreme climatic conditions. However, little is known about actual performance of plants at higher elevations. We analyzed 81 populations of Ranunculus kuepferi from the whole distribution area in the European Alps to quantify apomictic versus sexual seed formation via flow cytometric seed screening. Seed set and vegetative growth were measured as fitness parameters. All parameters were correlated to geographical distribution, elevation, temperature and precipitation. Flow cytometric seed screening revealed predominantly obligate sexuality $(88.9 \%)$ and facultative apomixis in diploid populations, while tetraploid populations are predominantly facultative $(65.4 \%)$ to obligate apomictic. Apomictic seed formation correlated significantly to higher tions, which explains also the observed niche shift to lower temperatures. However, within the totraploid range, tions, which explains also the observed niche shift to lower temperatures. However, within the tetraploid range, dipe is no applans cor apeared in diplois the ap a the lp Did observed. Polyploidy may rexuals have no fitness advantage. Instead, facultative apomixis may have aided colonization of higher elevations and range expansions in the Alps without mate and pollinator limitation, but did not necessarily involve long-distance dispersal. A direct influence of low temperatures on unreduced gamete formation cannot be ruled out.

Keywords: Apomixis; environmental gradients; fitness; flow cytometry; geographical parthenogenesis; polyploidy; Ranunculus kuepferi. 


\section{Abstract}

Apomictic plants expand their geographical distributions more to higher elevations compared to their sexual progenitors. It was so far unclear whether this tendency is related to mode of reproduction itself or represents a side effect of polyploidy. Apomixis is advantageous for range expansions as no mating partners and pollinators are needed (Baker's rule). Polyploidy is thought to infer fitness advantages and a higher vigour that would enable plants to adjust better to more extreme climatic conditions. However, little is known about actual performance of plants at higher elevations. We analyzed 81 populations of Ranunculus kuepferi from the whole distribution area in the European Alps to quantify apomictic versus sexual seed formation via flow cytometric seed screening. Seed set and vegetative growth were measured as fitness parameters. All parameters were correlated to geographical distribution, elevation, temperature and precipitation. Flow cytometric seed screening revealed predominantly obligate sexuality (88.9\%) and facultative apomixis in diploid populations, while tetraploid populations are predominantly facultative (65.4\%) to obligate apomictic. Apomictic seed formation correlated significantly to higher elevations, which explains also the observed niche shift to lower temperatures. However, within the tetraploid range, there is no apparent correlation of degree of facultative apomixis to geographical distance. Apomixis appeared in diploids three times independently in separated, otherwise sexual populations in the southwestern refugial areas of the Alps. Diploid apomixis was not successful in range expansions, and obligate sexual polyploids were not observed. Polyploidy may relate to cold tolerance as an adaptation to conditions at high elevations, where diploid sexuals have no fitness advantage. Instead, facultative apomixis may have aided colonization of higher elevations and range expansions in the Alps without mate and pollinator limitation, but did not necessarily involve long-distance dispersal. A direct influence of low temperatures on unreduced gamete formation cannot be ruled out.

\section{Introduction}

The term Geographical Parthenogenesis (GP) denotes a long known phenomenon that closely related sexual and apomictic taxa exhibit largely divergent distribution patterns (Vandel 1928). A number of recent studies have dealt with aspects of GP both in plants and animals. Distribution areas of asexual organisms generally seem to be larger than those of their sexual congeners, mostly expressing a tendency to marginal habitats such as higher latitudes 
and elevations as well as to disturbed areas (Bell 1982, Bierzychudek 1985, Van Dijk 2003, Kearney 2005, Hörandl 2006, 2009). However, the causal reasons for these shifts in distribution patterns are only poorly understood and have given rise to competing hypotheses.

Apomicts inherently possess the potential of founding new populations by only a single propagule or individual. Unlike their sexual relatives, apomictic plants do not necessarily require any pollinators or mating partners because they generate their seeds either independently of pollen (autonomous endosperm) or are able to use self-pollen for fertilization of the endosperm (pseudogamy); Hörandl (2010). Similar to the advantages of selfing, such uniparental reproduction is described in Baker's law as beneficial for colonization, and is expected to be most efficient after long-distance dispersal (Baker 1965, 1967, Pannell et al. 2015).

Although Baker's law explains well horizontal range expansions of apomictic plants (Baker 1955, 1967, Mogie and Ford 1988, Cosendai et al. 2013), it does not provide a theoretical background for the strong tendency of apomicts to colonize higher elevations and latitudes (Bierzychudek 1985, Asker and Jerling 1992). Alpine zones in high mountain systems represent extreme habitats for plant life, with lower temperatures including freezing, shorter vegetation periods and stronger wind exposure with increasing elevation (Nagy and Grabherr 2009). Under this aspect, different lines of hypotheses can be developed to specifically explain a preference of apomicts for higher elevations: first, apomictic plants are mostly polyploids (Carman 1997), and polyploidy by itself confers genomic features which could provide more physiological and ecological flexibility to adapt to harsh conditions. Polyploidy is characterized by higher allelic diversity, heterozygosity and often by heterosis effects (Birchler et al. 2010). Genome doubling can be accompanied by gene silencing, diversification in gene expression, differential expression of gene copies and epigenetic changes (Osborn et al. 2003, Adams and Wendel 2005, Comai 2005, Hegarty and Hiscock 2008). Epigenetic change and alterations in gene expression are important for the control of phenotypic plasticity and rapid adaptation (Nicotra et al. 2010). Accordingly, benefits of polyploidy could indirectly promote adaptation of apomicts to higher elevations (Bierzychudek 1985). However, little is known about reproductive fitness and vegetative performance under extreme alpine conditions (Wagner and Reichegger 1997, Wagner and Mitterhofer 1998, Kudo and Hirao 2005, Ladinig et al. 2013).

Second, a preference for extreme habitats could be explained by population genetic structure in apomictic taxa. The Frozen Niche Variation model (FNV) described by Vrijenhoek (1984, 
1994, Vrijenhoek and Parker 2009) postulates an advantage of apomicts based on their efficiency in niche exploitation. The model assumes that hybrid origin, autopolyploidization and facultative sexuality among the descendants constitute multiple discrete arrays of clones featuring different and diverse genotypes. Natural selection causes partitioning among the clonal lineages and accordingly, some apomictic genotypes may specialize on ecological niches outside the ecological optimum of the sexual parent(s). Clonal arrays do not only encompass most of the niche space of the sexual progenitor(s), but can also exploit the extreme range of the niche space more efficiently (Vrijenhoek and Parker 2009).

Beside these 'classical' explanations, the direct influence of climate is also brought into focus of the discussion. Cold stress is known to trigger formation of unreduced pollen (Ramsey and Schemske 1998, Bomblies et al. 2015), which is due to disturbance of microtubule formation at meiosis and defects in post-meiotic cytokinesis (De Storme et al. 2012). Unreduced pollen formation, however, is the major pathway leading to sexual polyploidization (De Storme et al. 2013). Female development, where the same principles may apply for the formation of unreduced embryo sacs, is even less understood because of methodological difficulties to study female meiosis. Furthermore, there is a lack of experimental work and quantitative data on these processes in natural populations of non-model species under alpine conditions. The alpine species Ranunculus kuepferi is a suitable model system for studying the correlations of mode of reproduction, polyploidy and elevation. The species has diploid and tetraploid cytotypes, with diploids occurring in the southwestern parts of the Alps, while tetraploids colonize the Northern, Central and Eastern Alps, Corsica and the Northern Apennines (Burnier et al. 2009, Cosendai and Hörandl 2010). Post-glacial colonization of the Alps happened probably out of the southwestern glacial refugia (Burnier et al. 2009). Previous analyses of spot samples on a few individuals suggested sexual seed formation in diploids, and facultative apomixis in tetraploids (Burnier et al. 2009, Cosendai and Hörandl 2010). However, these studies did not quantify pathways of seed formation, and hence could not test for statistical correlations to geographical distances, elevation and related environmental parameters. Population genetic studies suggested autopolyploid origin (Cosendai et al. 2011), a high genotypic diversity and lack of geographical structure among tetraploids (Cosendai et al. 2013). Self-fertility of tetraploids supported the assumption of Baker's law that rapid colonization could have played a role in distributions (Cosendai et al. 2013). Recently, Kirchheimer et al. (2016) found a niche shift between diploid and tetraploid cytotypes, mostly towards 
lower temperatures, but it remained unclear whether this shift actually correlates to mode of reproduction or to other physiological features connected to polyploidy. Shifts to lower temperatures in high elevations and northern latitudes are often connected to special morphological adaptations, like small growth form (alpine dwarfism), which is mostly due to slower cell cycle and cell differentiation processes (Körner 2003). Shifts to higher elevations, however, are also connected to additional physiological stress factors, like higher UV radiation, and lower $\mathrm{CO}_{2}$ atmospheric pressure, resulting in lower carbon availability (Körner 2003).

So far, it was unknown whether ploidy levels and mode of reproduction are strictly correlated, or whether sexual tetraploid or apomictic diploid plants do occur in natural populations. Kirchheimer et al. (2016) hypothesized that the observed niche shift may not be the decisive factor, but rather consequence of a rapid colonization process, which was enhanced by the ability of rapidly founding populations via apomixis. In the case of frequent founder events after long distance dispersal, apomictic seed production should be most frequent in the marginal populations of the distribution range. If the colonization process was mainly driven by the niche shift according the Frozen Niche Variation model, frequencies of apomixis should be positively correlated to the coldest locations, i.e. either in elevation or in latitude. However, no study has so far quantitatively compared the proportions of facultative sexual reproduction within and among populations at different elevations and in different parts of the distribution range. Moreover, the full range of pathways of seed formation possible in apomictic plants (Matzk et al. 2000, Dobeš et al. 2013), had never been assessed before in $R$. kuepferi. For instance, it was so far unknown to which extent tetraploid obligate sexuals or diploid apomicts would contribute to the distribution patterns. Here, we present a comprehensive dataset on reproductive pathways and fitness parameters of $R$. kuepferi from 81 populations out of the European Alps to test the following hypotheses: (1) Are mode of reproduction and ploidy level strictly correlated? (2) Is diploid apomixis or sexual polyploidy successful in range expansions? (3) Is there a correlation of apomictic mode of reproduction to higher elevation, or do we find a correlation to geographical distance? (4) Are there quantitative differences in seed set and in morphological fitness between cytotypes, and do they correlate to elevation? (5) Are there correlations of mode of reproduction to key climatic factors at higher elevations, i.e. temperature and precipitation? A more comprehensive study on the effects of climate factors and niche dynamics on cytotypes has been presented elsewhere (Kirchheimer et al. 2016). 


\section{Methods}

\section{a) Plant material}

Plants of Kuepfer's buttercup (Ranunculus kuepferi) have been collected throughout the Alps from 81 populations during two consecutive summer periods in 2013 and 2014 [see Supporting Information - Table S1]. We accessed all published localities (Burnier et al. 2009, Cosendai and Hörandl 2010) as well as records from herbaria and from the floristic literature. At the sites, we randomly selected a $100 \mathrm{~m} \times 100 \mathrm{~m}$ plot to define a population. Apart from three exceptions where less than five plants were found, we sampled 12 individuals per population (1074 in total) in the post-anthesis to the early fruiting stage. Microscopic investigations on ovule development (Burnier et al. 2009, C. Schinkel unpubl. data, following methods of Hojsgaard et al. 2014) confirmed that ovule development happens in R. kuepferi during the very early bud stage. All buds collected in the wild already showed fully mature female gametophytes and represent the 7-celled, 8 nucleate Polygonum-type embryo sac, as typical for $R a$ nunculus (Nogler 1984, Hojsgaard et al. 2014). This fits to general observations that alpine plants produce floral primordia in the year before, and finish ovule development in buds below ground before sprouting (Körner 2003, Nagy and Grabherr 2009). Hence, we can assume that sexual vs. apomictic developmental pathways in $R$. kuepferi were already completed under natural conditions before collection of plants, and only ripening of seeds happened under garden conditions. Plants were taken from four $2 \mathrm{~m} \times 2 \mathrm{~m}$ randomly chosen subplots (Kirchheimer et al. 2016). All plants were dug out, transported to the Botanical Garden of the University of Goettingen, and cultivated in pots. Single fruiting heads were bagged with perforated plastic pouches to harvest all mature achenes of a collective fruit. Achenes were kept for at least 10 days at room temperature, before bundled in paper bags and stored on silica gel at $8^{\circ} \mathrm{C}$ for later analyses.

\section{b) Flow cytometric seed screen (FCSS) and ploidy determination}

Like many other facultative apomicts, a single plant can produce both sexual and apomictic seeds within the same flower (Aliyu et al. 2010, Dobeš et al. 2013). To quantify the main mode of reproduction, we determined ploidies of both endosperm and embryo per single seed for each individual. Since many tetraploid plants had a poor seed set, as reported previously (Huber 1988, Cosendai and Hörandl 2010), we had to restrict the sampling to 551 individuals, 
which formed each a minimum of five well-developed seeds per flower. Five seeds per plant from at least three plants per population were analyzed with a slightly modified FCSS method according to Matzk et al. (2000). Seeds were placed in $2 \mathrm{ml}$ Eppendorf tubes together with two $0.23 \mathrm{~cm}$ steel beads (QIAGEN, Hilden, Germany) and ground in a TissueLyser II mill (QIAGEN, Hilden, Germany) with a stroke rate of $30 \mathrm{~Hz}$ for $7 \mathrm{~s}$. Further preparation was realized using a two-step procedure described by Doležel et al. (2007) performing (1) a nuclei isolation step with Otto I buffer: $0.1 \mathrm{M}$ citric acid monohydrate, $0.5 \%$ v/v Tween 20 (Sigma-Aldrich Munich, Germany), ddH $\mathrm{H}_{2} \mathrm{O}$ and (2) a separate staining step with Otto II buffer: $0.4 \mathrm{M}$

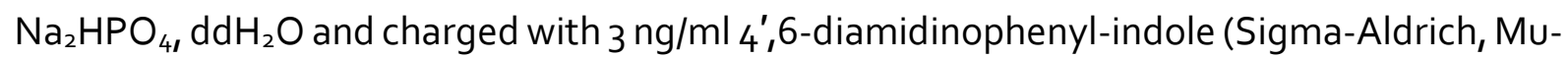
nich, Germany). Macerated seeds were incubated for 5 minutes with $200 \mu$ lice-cold Otto I buffer. Suspensions were filtered through $40 \mu \mathrm{m}$ mesh tubes (Partec, Münster, Germany). $800 \mu$ l Otto II buffer were then added and incubated for another 15 minutes before analysis. Ploidy levels of all mother plants were determined on fresh leaves from the cultivated plants using the same methods as described above, except for a slightly prolonged grinding time in the Tissuelyser (15 s).

All analyses were performed on a CyFlow Space flow cytometer (Partec, Münster, Germany). Histograms were taken and analyzed with the supplied FloMAX Software version 2.2.0 (Quantum Analysis GmbH, Münster, Germany). Leaf material of Zea mays (CE-777 strain, provided by Doležel J.) and a diploid tested plant of $R$. kuepferi were used as external reference standard to adjust the gain level of the UV LED lamp. All subsequent analyses were conducted with the same parameters.

Peak ranges for embryo (em) and endosperm (es) were set manually in FloMAX and values of DNA content were calculated as Gaussian means. Ratios of es:em ploidies were calculated to determine whether a seed has been produced sexually (3:2 ratio) or via apomixis (3:1, 2.5:1, 2:1 ratio). Interpretation of all plausible pathways for development and fertilization of seeds of R. kuepferi (Table 1) have been adopted from the studies by Matzk et al. (2000), Talent and Dickinson (2007) , Cosendai and Hörandl (2010) and Dobeš et al. (2013), and provided the basis for our classification; terminology for designation of ploidy levels follows Greilhuber et al.(2005). A threshold of 1.65 es:em ratio was set to discriminate between sexual (lower valves) and asexual (higher values) cases. Those with ratio values between 1.85 and 2.15 were interpreted as autonomous endosperm development since the second peak was always dis- 
tinct and as high as the endosperm peak in other pathways. Hence, we interpreted it as endosperm peak, and we excluded the possibility that it could represent just a G2 peak of the growing embryo ( $G_{2}$ peaks are usually much smaller than the respective $G_{1}$ peak, as only few cells are in the respective stage of the cell cycle). Representative flow cytometric histograms are shown in Supporting Information - Figure $\mathrm{S}_{1}$.

We categorized every individual and population as obligate sexual (only sexual seeds), obligate apomictic (only apomictic seeds) or mixed (sexual as well as apomictic seeds = facultative apomixis) by pooling the results of the analyzed seeds. Since we had just a sampling of $n$ = 5 seeds per individual, we did not calculate individual-level percentages, but just recorded the category for each plant. Instead, we pooled all seeds per population for calculating percentages and correlations of mode of reproduction with other variables. 
Table 1 Observed pathways of seed formation in Ranunculus kuepferi.

\begin{tabular}{|c|c|c|c|c|c|c|c|}
\hline & & \multicolumn{3}{|l|}{$\mathrm{N}$} & \multicolumn{2}{|l|}{ Ploidy } & \multirow{2}{*}{$\begin{array}{l}\text { Genome contribution of sperm nuclei to endo- } \\
\text { sperm }\end{array}$} \\
\hline & & seed & polar nuclei & sperm nuclei & Embryo & Endosperm & \\
\hline \multicolumn{8}{|l|}{ Diploid } \\
\hline Sexual & $A$ & 663 & 2 & 1 & ${ }_{1} C x(m)+1 C x(p)$ & $2 C x(m)+1 C x(p)$ & 1 reduced \\
\hline \multirow[t]{2}{*}{$B_{\text {III }}$} & $A B$ & 4 & 2 & 1 or 2 & $\begin{array}{l}2 \mathrm{Cx}(\mathrm{m})+1 \mathrm{Cx}(\mathrm{p}) / 2 \mathrm{Cx}(\mathrm{m})+ \\
2 \mathrm{Cx}(\mathrm{p})\end{array}$ & $\begin{array}{l}4 C x(m)+1 C x(p) / 4 C x(m)+ \\
2 C x(p)\end{array}$ & 1 reduced/1 unreduced \\
\hline & $\mathrm{A}_{2}$ & 30 & 2 & 1 & $2 \mathrm{Cx}(\mathrm{m})$ & ${ }_{4} C x(m)+1 C x(p)$ & 1 reduced \\
\hline \multirow[t]{2}{*}{ Asexual } & $A_{3}$ & 2 & 2 & 1 or 2 & $2 \mathrm{Cx}(\mathrm{m})$ & $4 C x(m)+2 C x(p)$ & 2 reduced or 1 unreduced \\
\hline & $\mathrm{A}_{4}$ & 2 & 2 & 2 & $2 C x(m)$ & ${ }_{4} C x(m)+3 C x(p)$ & 2 reduced $(\sim 1.5 C x) *$ \\
\hline \multicolumn{8}{|l|}{ Triploid } \\
\hline Sexual & B & 6 & 2 & 1 & $\begin{array}{l}{ }_{1} C x(m)+2 C x(p) / 2 C x(m)+ \\
{ }_{1} C x(p)\end{array}$ & $\begin{array}{l}2 C x(m)+2 C x(p) / 4 C x(m)+ \\
1 C x(p)\end{array}$ & 1 reduced (diploid/haploid sperm nuclei) \\
\hline \multirow[t]{3}{*}{$\mathrm{B}_{\| I} * * *$} & BB & 6 & 2 & 2 & $3 C x(m)+1 C x(p)$ & $\begin{array}{l}6 C x(m)+6 C x(p) / 6 C x(m)+ \\
{ }_{4} C x(p) / 6 C x(m)+2 C x(p)\end{array}$ & $\begin{array}{l}\text { endosperm polyploidization } 2 \text { reduced (dip- } \\
\text { loid/haploid sperm nucleus) }\end{array}$ \\
\hline & $\mathrm{B} 2$ & 101 & 2 & 1 or 2 & $3 C x(m)$ & $\begin{array}{l}6 C x(m)+2 C x(p) / 6 C x(m)+ \\
{ }_{1} C x(p)\end{array}$ & 1 reduced (diploid/haploid sperm nucleus) \\
\hline & B3 & 12 & 2 & 1 or 2 & $3 C x(m)$ & $\begin{array}{l}6 C x(m)+{ }_{5} C x(p) / 6 C x(m)+ \\
{ }_{4} C x(p)\end{array}$ & 2 reduced $(\sim 2.5 C \mathrm{C}) * / 2$ reduced \\
\hline \multirow[t]{3}{*}{ Asexual } & $\mathrm{B}_{4}$ & 2 & 2 & 1 or 2 & $3 C x(m)$ & $6 C x(m)+3 C x(p)$ & 1 unreduced or 2 reduced $(\sim 1.5 C x)^{*}$ \\
\hline & B5 & 2 & 2 & 2 & $3 C x(m)$ & $6 C x(m)+6 C x(p)$ & 2 unreduced \\
\hline & $\mathrm{D}_{1}$ & 2 & 2 & 1 & $3 C x(m)$ & $12 C x(m)+3 C x(p)$ & endosperm polyploidization +1 unreduced \\
\hline
\end{tabular}




\begin{tabular}{|c|c|c|c|c|c|c|c|}
\hline \multicolumn{8}{|c|}{ Tetraploid } \\
\hline Sexual & $\mathrm{C}$ & 118 & 2 & 1 & $2 C x(m)+2 C x(p)$ & $4 C x(m)+2 C x(p)$ & 1 reduced \\
\hline \multirow[t]{4}{*}{$\mathrm{B}_{\text {III }}$} & $\mathrm{CB}$ & 33 & 2 & 1 or 2 & $\begin{array}{l}{ }_{4} \mathrm{Cx}(\mathrm{m})+2 \mathrm{Cx}(\mathrm{p}) / 4 \mathrm{Cx}(\mathrm{m})+ \\
{ }_{4} \mathrm{Cx}(\mathrm{p})\end{array}$ & $\begin{array}{l}8 \mathrm{Cx}(\mathrm{m})+2 \mathrm{Cx}(\mathrm{p}) / 8 \mathrm{Cx}(\mathrm{m})+ \\
{ }_{4} \mathrm{Cx}(\mathrm{p})\end{array}$ & 1 unreduced $/ 2$ reduced \\
\hline & $\mathrm{C}_{2}$ & 1258 & 2 & 1 & ${ }_{4} \mathrm{Cx}(\mathrm{m})$ & $8 C x(m)+2 C x(p)$ & 1 reduced \\
\hline & $\mathrm{C}_{3}$ & 400 & 2 & 1 or 2 & $4 \mathrm{Cx}(\mathrm{m})$ & $8 C x(m)+4 C x(p)$ & 2 reduced or 1 unreduced \\
\hline & $\mathrm{C}_{4}$ & 58 & 2 & 2 & ${ }_{4} \mathrm{Cx}(\mathrm{m})$ & $8 C x(m)+6 C x(p)$ & 2 reduced $(\sim 3 C x)^{*}, * *$ \\
\hline \multirow[t]{5}{*}{ Asexual } & $C_{5}$ & 24 & 2 & 2 & $4 \mathrm{Cx}(\mathrm{m})$ & $8 C x(m)+8 C x(p)$ & 2 unreduced or endosperm poyploidization, $* *$ \\
\hline & C6 & 16 & 2 & 0 & ${ }_{4} \mathrm{Cx}(\mathrm{m})$ & $8 C x(m)$ & autonomous endosperm \\
\hline & $D_{2}$ & 25 & 2 & 1 & ${ }_{4} \mathrm{Cx}(\mathrm{m})$ & $8 C x(m)+1 C x(p)$ & 1 reduced diploid \\
\hline & $D_{3}$ & 35 & 2 & 1 or 2 & ${ }_{4} \mathrm{Cx}(\mathrm{m})$ & $8 C x(m)+3 C x(p)$ & 1 reduced $(\sim 3 C x) *$ \\
\hline & $\mathrm{D}_{4}$ & 4 & 2 & 1 & ${ }_{4} \mathrm{Cx}(\mathrm{m})$ & $16 C x(m)+4 C x(p)$ & endosperm polyploidization +1 unreduced \\
\hline
\end{tabular}

Cx, ploidy after DNA content (Greilhuber et al. 2005); m, maternal genome contribution; p, paternal genome contribution

* after unbalanced pollen meiosis

** also trinucleate endosperm possible $\left(C_{4}: 12 \mathrm{Cm}+{ }_{2} \mathrm{Cp}, \mathrm{C}_{5}: 12 \mathrm{Cm}+{ }_{4} \mathrm{Cp}\right.$; see also Talent and Dickinson (2007)

$* * *$ only possible variants of observed cases presented 


\section{c) Seed set and morphological fitness parameters}

Seed set was determined as percentage of all mature achenes per individual and measured as the total number of well-developed over the total number of achenes per flower per plant (Cosendai and Hörandl 2010). Discrimination between filled and empty achenes was done manually using a forceps. Filled ones have a well-developed endosperm and withstand applied pressure, while undeveloped ones collapse easily.

As a measurement for individual vegetative fitness, we quantified number and length of leaves (longest leaf per rosette) and shoots as well as the number of buds, flowers and fruits of each plant before collection directly in the field (i.e. under natural conditions). Measurements served as a proxy for comparisons between cytotypes and for estimating the presence of heterosis in polyploids.

\section{d) Statistical analyses}

All analyses were conducted in R version 3.1.2 ( $R$ Core Team 2014) using the external packages R Commander (Rcmdr) version 2.1-5 (Fox 2005) and lattice version 0.20-29 (Sarkar 2008).

Percentages were arcsine transformed, all other variables were converted to the natural logarithm before analysis to improve normal distribution of the data. Seed set was determined per flower and subsequently averaged per plant. To identify effects of ploidy and selected ecological parameters (WorldClim) on distribution differences and reproductive mode variances between cytotypes, we conducted one-way ANOVA on population scale, with ploidy and the respective analysis dependent predictor as fixed effect. Seed set differences between cytotypes were equally analyzed with ANOVA, and ploidy as well as the respective ecological predictors as fixed effects. Further, correlations among ecological predictors and reproduction mode, seed set, ploidy were tested on population scale with Spearman's rank-order correlation.

\section{e) Environmental factors}

We tested correlations of elevation and several climatic variables at the sites of plant origin, i.e. our sampling sites, on cytotypes and modes of reproduction. Data for elevation were taken from the collection sites with the barometric altimeter of an eTrex 30 GPS device (Garmin Deutschland GmbH, Garching, Germany) (see Appendix A). Climatic variables were 
downloaded from WorldClim (www.worldclim.org) with a spatial resolution of approximately $1 \mathrm{~km}^{2}$ (Hijmans et al. 2005). To fit climate data to our sample-plot size, WorldClim variables have been statistically downscaled to a resolution of $100 \mathrm{~m} \times 100 \mathrm{~m}$ (for detailed description see Kirchheimer et al. 2016). From the provided 19 bioclimatic variables within the dataset, we chose $\mathrm{BIO}_{1}$ as annual mean temperature, $\mathrm{BIO}_{7}$ as temperature annual range (maximumminimum temperature), $\mathrm{BIO} 10$ as mean temperature of warmest quarter and $\mathrm{BIO}_{12}$ as annual precipitation - which represent the most important climatic drivers of plant growth - for our analysis.

\section{Results}

A total of 551 individuals from 81 populations provided sufficient material for analyses. Flow cytometry of leaves revealed 132 diploid 25 triploid and 394 tetraploid individuals. Most populations were of uniform cytotype: we discovered 18 diploid (22.2\%) and 52 (64.2\%) tetraploid populations, while 11 (13.6\%) populations had a mixed composition of different cytotypes ranging from di- to even pentaploid plants. Triploid individuals were found scattered across mixed populations in the contact zone of diploids and tetraploids (Fig. 1) but did not constitute any single uniformly triploid population.

Surprisingly, flow cytometric seed screening revealed that only a minority of 34 (42.0\%) populations reproduced uniformly (obligate sexual or apomictic seed formation), while 47 populations (58.0 \%) had a mixed mode of reproduction (i.e. both sexual and apomictic seed formation). We excluded seeds from three pentaploid and one hexaploid individuals from further analysis. Seeds formed via partial apomixis ( $B_{\| I}$ hybrids, see below), were analyzed but also excluded from further statistical analysis.

\section{a) Modes of seed formation}

The analysis of a total of 2795 seeds revealed in general a noticeably high variability in seed formation among all plants studied (Table 1). In diploids, sexuality was by far the most prevalent mode of reproduction. The majority of seeds ( $94.7 \%$ from 700 in total) were derived sexually. In each case, we observed embryo to endosperm ratios of $2 \mathrm{C}: 3 \mathrm{C}$, which is characteristic for sexual development. 


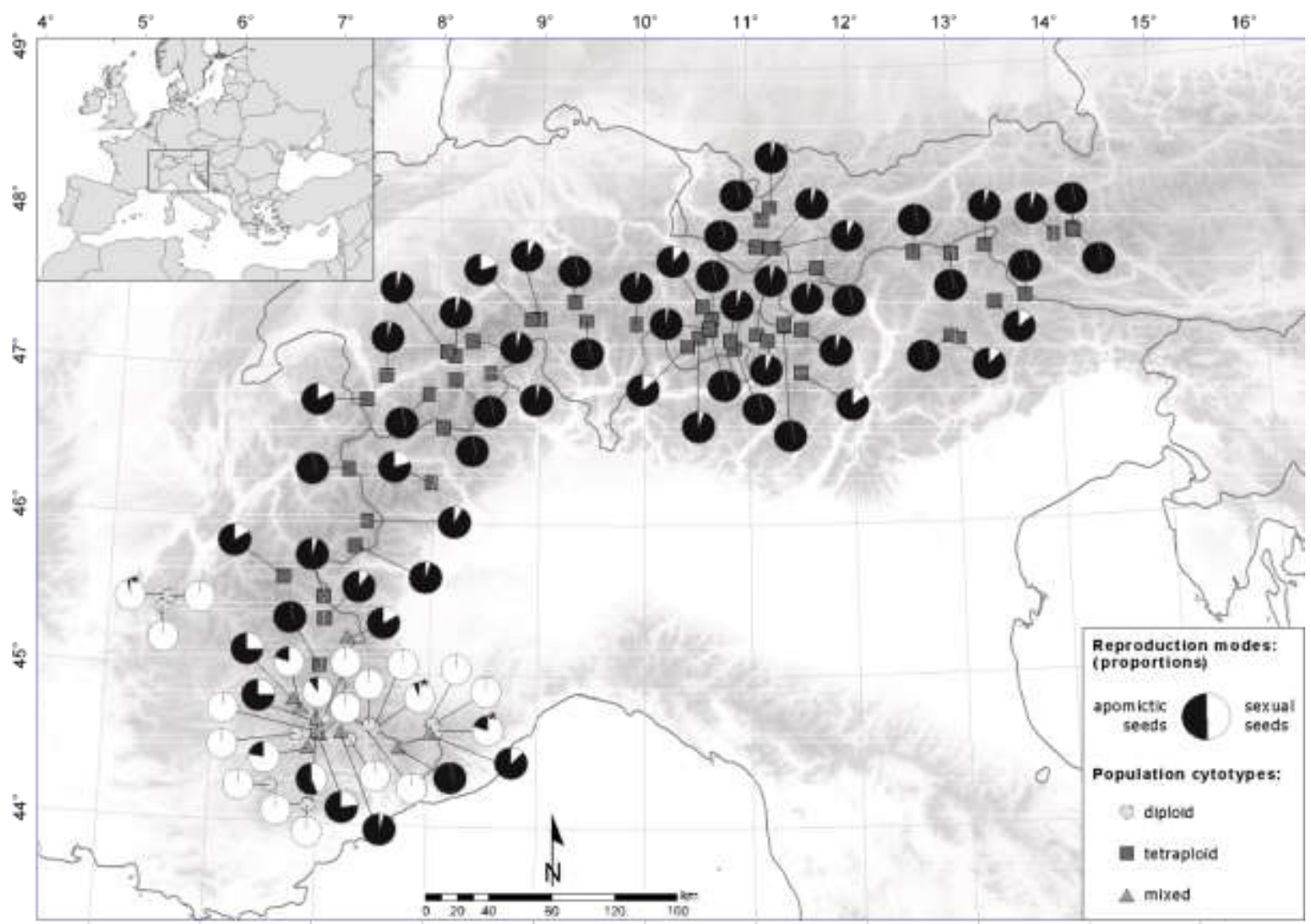

Figure 1. Geographical distribution of Ranunculus kuepferi reproduction modes of analyzed populations on a map of the European Alps with elevation model. Pie diagrams indicate proportions of sexual (white) and apomictic seed (black) formation within the populations, diploids with apomicts are marked by an asterisk. Cytotypes: $2 \times$, diploid populations; $4 \times$, tetraploid populations; mixed, populations consisting of two or more different cytotypes, including triploids.

Normal fertilization with a reduced pollen results in a fertilized diploid embryo $\left(1 \mathrm{Cm}+{ }_{1} \mathrm{Cp}\right)$ and a triploid endosperm formed by the fusion of two reduced maternal central nuclei subsequently fertilized with a second pollen sperm nucleus $(1 \mathrm{Cm}+1 \mathrm{Cm}+1 \mathrm{Cp}$; pathway $A$ in Table 1, [see Supporting Information-Fig. S1a]). Nonetheless, a tiny fraction of $4.9 \%$ (34) of diploid seeds originated via apomixis (pathways $A_{2}-A_{4}$ in Table 1, [see Supporting Information-Fig. $\mathrm{S}_{1}$ b]). Autonomous endosperm development in diploids $(2 \mathrm{Cm}+2 \mathrm{Cm}$ ) was not observed. Instead, we recorded three cases (0.4\%) of ploidy shifts in the embryo among diploid apomictic seeds, originating from fertilization of unreduced egg cells (pathway AB). Although rather rare, principally all cytotypes were prone to that, resulting in the formation of so-called BIII-hybrids (Nogler 1984), which exhibit partial apomixis, i.e. a combination of apomeiosis and fertilization. Due to the availability of maternal leaf ploidy data for all plants, $B_{I I I}$ hybrids (embryos with higher ploidy than the mother plant) could be determined with great certainty. 
Tetraploid plants also exhibited a profound level of variance in mode of reproduction. While their primary reproduction mode was conversely based on apomictic pathways that account for $91.2 \%$ (1797) of the yielded seeds (pathways C2-D4), a total of $6.0 \%$ (118) were developed sexually (pathway $C_{i}$ [see Supporting Information-Fig. S1c]). Beside some ambiguous cases, apomictic seeds could be assigned to nine different developmental pathways (Table 1). With $99.2 \%$ (1782), the vast majority of apomictic seeds were formed pseudogamously. Unreduced pollen should result in highly polyploid endosperm (12C or 16C), depending on whether one or both sperm nuclei were used (pathways $C_{3}$ or $C_{5}$ respectively; [see Supporting Information-Fig. S1d-f]), while reduced pollen can fertilize the endosperm with either one (10C) or two $(12 C)$ of its sperm nuclei. Alternatively, in some cases also trinucleate endosperm can explain the pattern (Talent and Dickinson 2007, see Table 1). A total amount of 16 seeds (0.8 $\%$ ) exhibited an octoploid endosperm, indicating autonomous formation from unreduced central cells (8C); pathway C6. $B_{\text {III }}$ hybrids with a shift to higher ploidy levels in the embryo were discovered in 43 seeds (2.2\%); pathway CB. In 21 cases (1.1\%), the pathways could not be reconstructed.

In triploid plants 119 seeds were produced by apomictic pathways (95.2\%; pathways B2-D1), only six ( $4.8 \%)$ were derived sexually. Within the apomictic pathways, two seeds were found with a diploid embryo, while three seeds contained tetraploid embryos. Autonomous endosperm formation was not observed.

\section{b) Variance and geographical pattern of reproduction mode of popula- tions}

Based on the individually pooled FCSS data, all plants could be assigned to three distinct groups. Most individuals $(450,81.7 \%$ ) reproduce uniformly, being either obligate sexual or obligate apomictic. The remaining 101 individuals (18.3\%) are facultatively apomictic (= mixed). In the 132 diploids, beside the typically obligate sexual plants (95.4\%), we found six individuals ( $4.6 \%$ ) being facultative apomictic, but no individual with obligate apomixis. The facultative apomictic diploids appeared in three geographically isolated populations (Fig. 1). Tetraploids were predominantly obligate apomictic, 90 plants (22.8\%) exhibited facultative apomixis to varying degrees, but none of the tetraploid individuals was obligate sexual. In triploids, five individuals (20.0\%) were discovered being facultative sexuals, while their main reproductive mode is based on obligate asexual pathways, like in tetraploids. 
Among diploid populations 15 were found to be obligate sexual (88.9\%) whereas three contained individuals with a mixed formation of seeds. In contrast, tetraploids exhibited 34 (65.4 $\%)$, a significantly greater proportion of populations with facultative sexuality $[t(70)=4.5, P<$ 0.01]. Only 18 populations were found to be obligate apomictic. Facultative apomixis therefore exhibits a significant correlation to ploidy $[F(2,78)=379.7, P<0.01]$. The 10 populations comprising varied cytotypes all express a mixed mode of reproduction. Overall, the occurrence of facultative sexuality is evenly scattered across populations and spread over the entire Alps in a seemingly random matter (Fig. 1). The Mantel test did not indicate a correlation of mode of reproduction and geographical distance (Observation $=0.016 ; P=0.17$ ).

\section{c) Seed set and morphological fitness parameters}

Variation in seed production of $R$. kuepferi was remarkably high among all three cytotypes. The highest variance was found in diploids with values ranging from $7.2 \%$ to $96.7 \%$ fully developed seeds. Within tetraploids, values varied between 4.1 and $83.3 \%$. On average, tetraploids produced $30.8 \% \pm 13.4$ well-developed seeds ( $\sim 15$ seeds: plant) and therefore showed a significantly lower proportion $[t(522)=-9.6, P<0.01]$ than diploids $(46.8 \% \pm 24.1, \sim 20$ seeds: plant). Triploids showed a lesser variance (5.8-50.8\%) but the lowest mean (25.8\% \pm 10.4) of all three groups. Hence, triploids differed significantly from diploids $[t(154)=-5.8, P$ $<0.01]$ but not from tetraploids $[t(418)=-1.5, P=0.29]$. Focusing on the reproduction mode, obligate sexual individuals (mean $47.0 \% \pm 24.1$ ) had a significantly higher seed set than obligate apomicts $[t(446)=9.3, P<0.01]$ and facultative sexual individuals $[t(224)=7.3, P<0.01]$. Analysis of data at the population level revealed similar results: uniformly diploid populations had the highest seed set (mean $41.9 \% \pm 17.6$ ), uniformly tetraploid populations produced significantly $[t(70)=-4.5, P<0.01]$ fewer filled achenes (mean $27.1 \% \pm 9.2$ ) and mixed cytotype populations performed worst (mean $23.4 \% \pm 10.9$ ), as they comprised plants of tetraand higher ploidies beside triploids and only a few diploid samples.

Morphometric data showed that diploid individuals tended to be taller than tetraploids $[t(1044)=2.8, P<0.01 ;$ Fig. 2a]. Leaf length did not differ significantly between cytotypes $[F(21125)=2.5, P<0.07]$, with diploids and tetraploids being particularly similar $[t(1043)=$ $0.3, P=0.94 ;$ Fig. 2b]. Diploids developed significantly more flowers $[t(1045)=3.7, P<0.01 ;$ Fig. $2 \mathrm{c}]$ and more leaves $[t(1045)=9.3, P<0.01$; Fig. $2 \mathrm{~d}]$ than tetraploids (Fig. $2 \mathrm{C}$ ). Moreover, for each of the two factors a negative correlation to elevation was found in diploids $\left[r_{s}(81)=\right.$ 
$-0.15, P=0.01$ and $\left.r_{s}(81)=-0.13, P=0.03\right]$. Triploids had an intermediate phenotype with significantly more leaves $[t(872)=3.1, P<0.01]$ and flowers $[t(872)=1.9, P<0.01]$ than tetraploids, but less leaves $[t(343)=-2.5, P=0.03]$ and flowers $[t(343)=-0.4, P=0.91]$ than diploids.
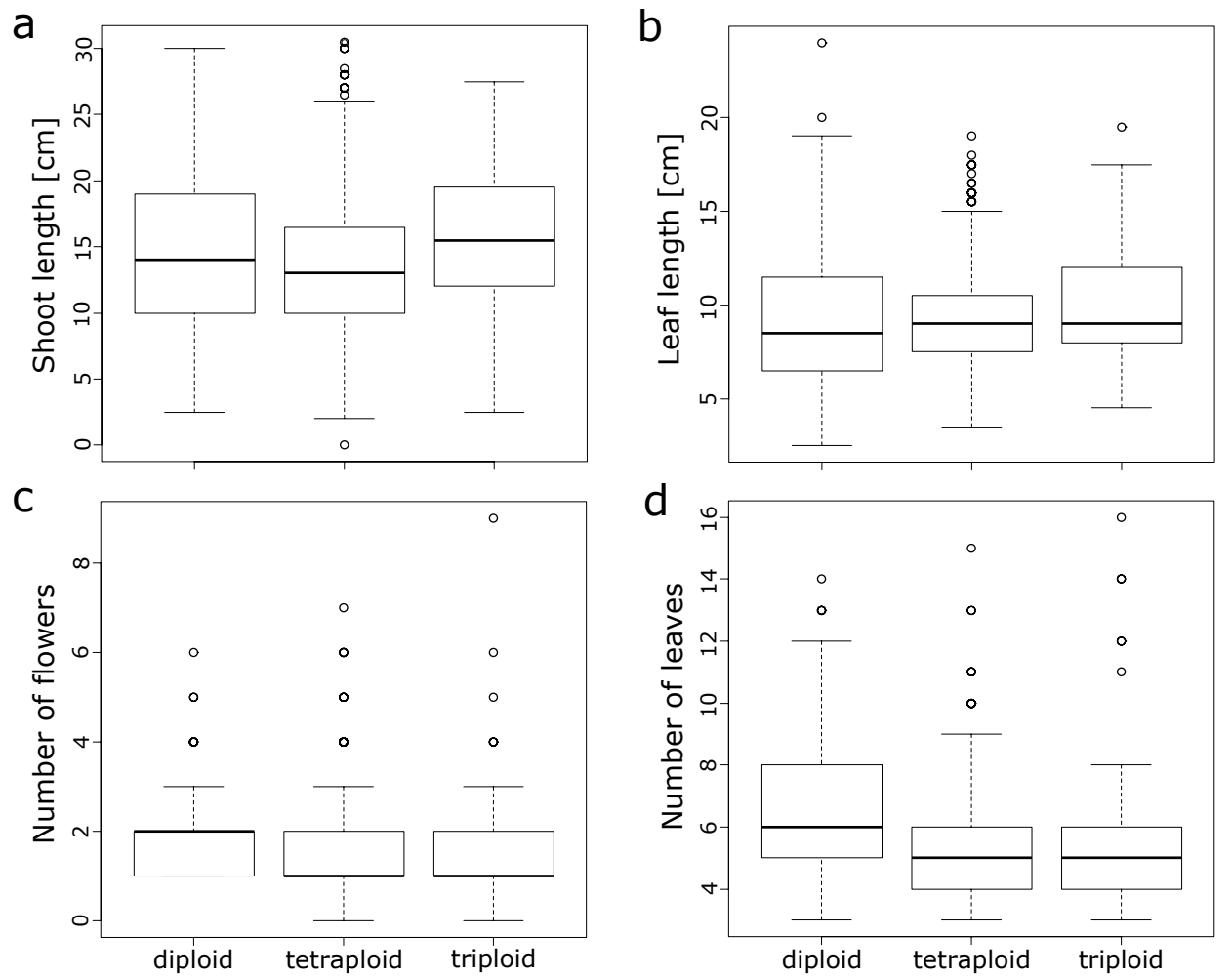

Figure 2. Boxplots of field collected fitness parameters on individual level of Ranunculus kuepferi cytotypes. Outliers are presented as black circles (o). (a) Total length of main shoots from ground to flower base, respectively fruit base. (b) Length of the longest leaf per plant. (c) Total number of flowers, respectively buds or fruits, per plant. (d) Total number of ground and shoot leaves per plant.

\section{d) Ecological factors}

Elevation of sites was significantly positively correlated to ploidy $\left[r_{s}(81)=-0.56, P<0.01\right]$. Tetraploid populations predominantly occurred at higher elevations (mean $2243.9 \mathrm{~m}$ a.s.l. \pm 70.6) than diploid (mean $1855.6 \mathrm{~m}$ a.s.l. \pm 245.7 ) and mixed ones (mean $1942.6 \mathrm{~m}$ a.s.l. \pm 251.7 ) (Fig. 3). Regarding reproductive fitness, diploids exhibited a strong negative correlation of seed set to elevation $\left[r_{s}(18)=-0.68, P<0.01\right]$, while tetraploid and mixed populations did not. The amount of facultative sexuality showed to be correlated to ploidy $\left[r_{s}(81)=-0.45, P<\right.$ $0.01]$. 


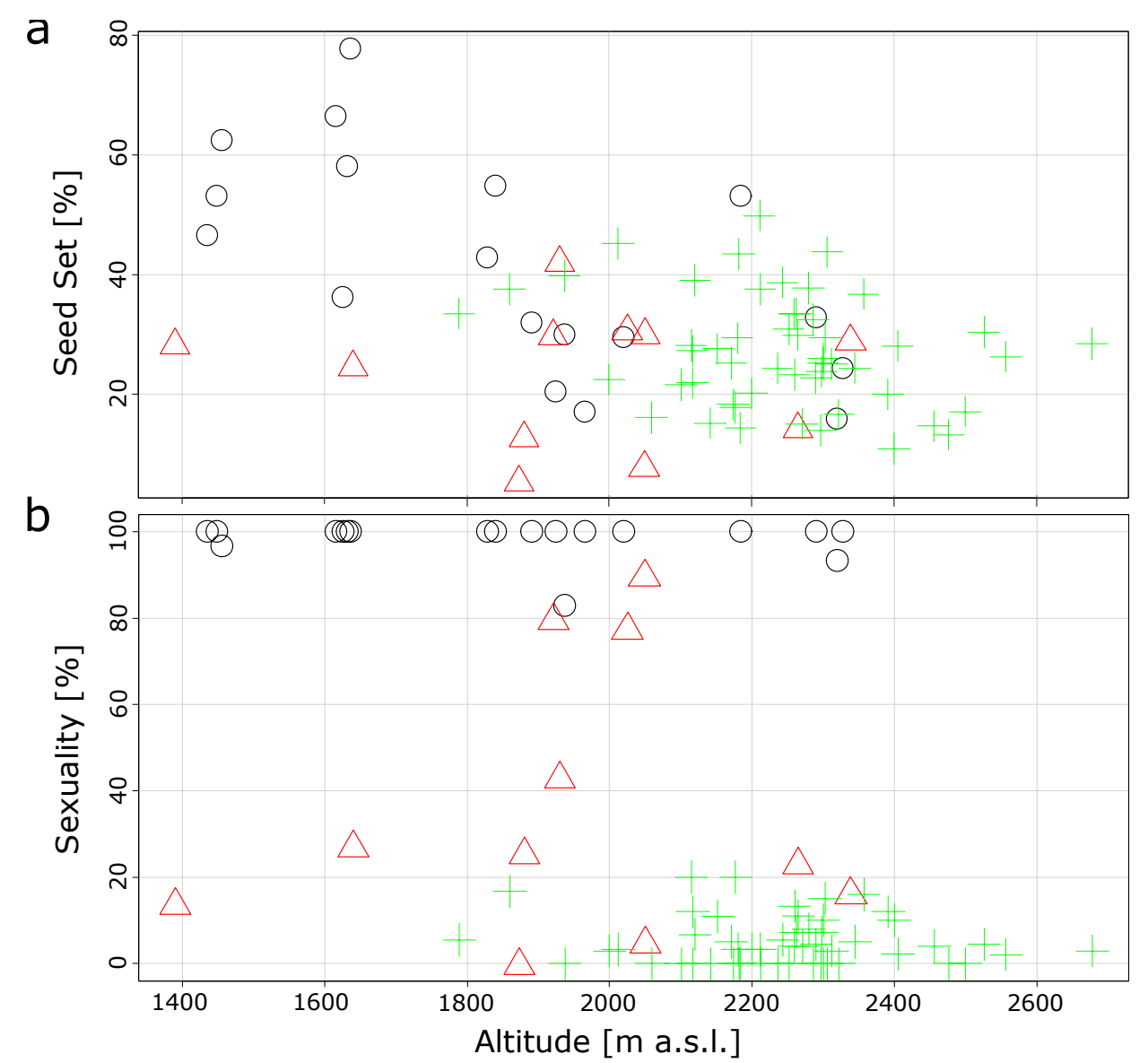

Figure 3. Scatterplots showing variation between Ranunculus kuepferi cytotypes on population level regarding reproduction parameters in dependence of elevation. Black circle (o): diploid populations; green cross (): tetraploid populations; red triangle (): population consisting of individuals with different cytotypes (mixed). (a-b) Proportions of well-developed achenes per flower and plant (seed set, a), as well as proportions of facultative sexuality contributing to seed development (b) in dependence of elevation above sea level (elevation).

However, the temperature of the warmest quarter is closely negatively correlated to elevation $\left[r_{s}(81)=-0.85, P<0.01\right]$ and hence similarly to seed set $\left[r_{s}(81)=0.33, P<0.01\right]$ and reproduction mode $\left[r_{s}(81)=-0.68, P<0.01\right]$. Average mean temperatures of the warmest quarter at sites were lowest among tetraploids $\left(7.3^{\circ} \mathrm{C} \pm 0.9\right)$, but did not differ significantly between diploid $\left(11.8^{\circ} \mathrm{C} \pm 1.4\right)$ and mixed populations $\left(11.3^{\circ} \mathrm{C} \pm 1.6\right)$ (Fig. $4 \mathrm{a}$ and $\mathrm{C}$ ).

Precipitation was significantly negatively correlated to proportion of facultative sexual individuals within a population $\left[r_{s}(81)=-0.40, P<0.01\right]$, and was highest at the sites of tetraploids (mean $119.1 \mathrm{~mm} \pm 18.0$ ). Here too, diploid (mean $96.2 \mathrm{~mm} \pm 8.6$ ) and mixed populations (mean $99.4 \mathrm{~mm} \pm 9.8$ ) did not differ significantly from each other. Seed set was not related to this variable $\left[r_{s}(81)=0.004, P=0.97\right]$ (Fig. $4 \mathrm{~b}$ and $\mathrm{d}$ ). Precipitation was positively correlated with ploidy $\left[r_{s}(81)=-0.48, P<0.01\right]$. 

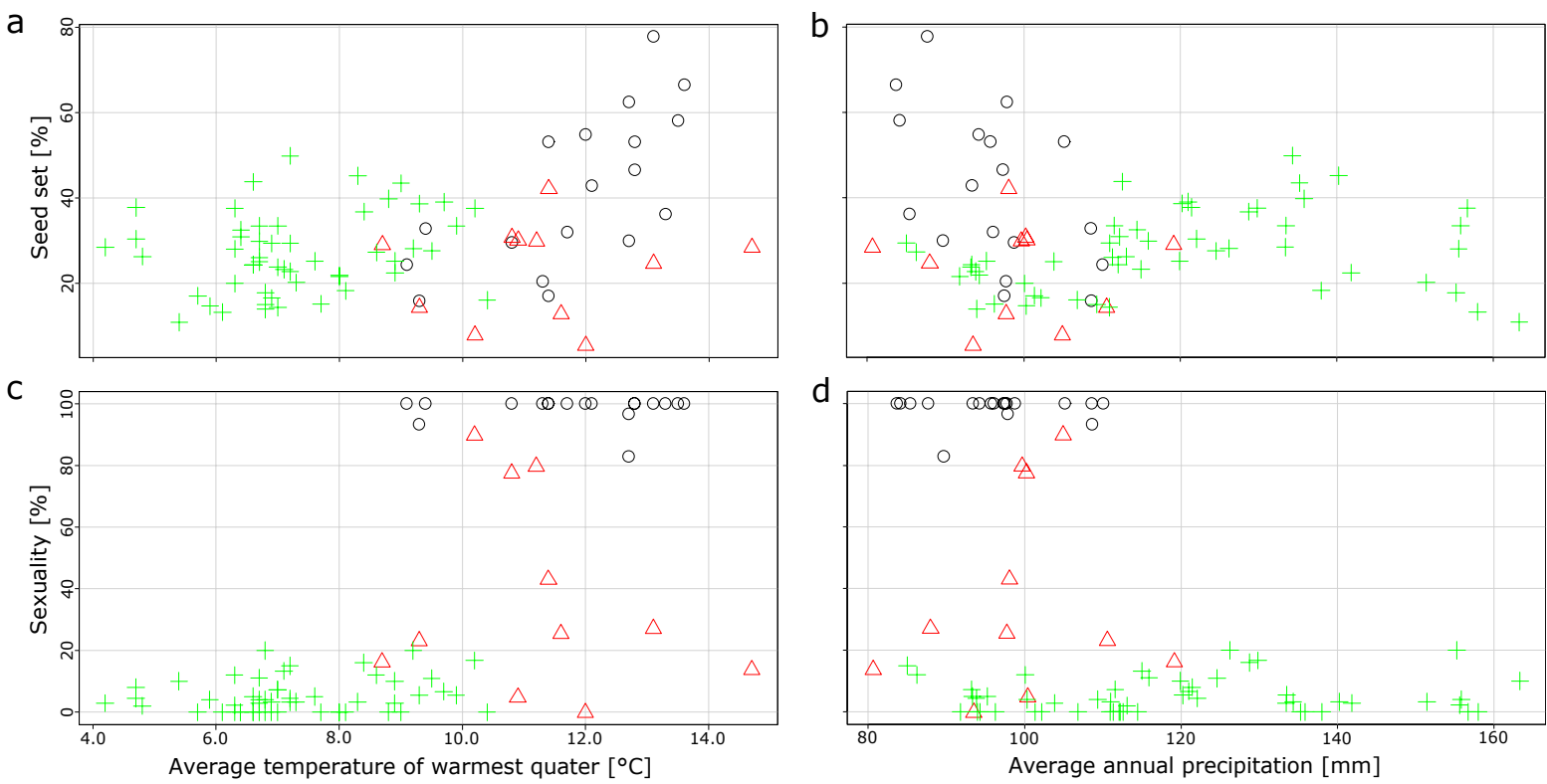

Figure 4. Scatterplots of ecological against developmental parameters on population level of Ranunculus kuepferi differentiated by cytotype. Black circle (o): diploid populations; green cross (): tetraploid populations; red triangle (): population consisting of individuals with different cytotypes. (a-b) Proportions of well-developed achenes averaged per flower and plant (seed set) in dependence of average temperatures of the three warmest months ( $\mathbf{a}, \mathrm{BIO} 10$, WorldClim) as well as in dependence of the average annual precipitation (b, BIO12, WorldClim). (c-d) Facultative sexuality in dependence of average temperatures (c) and in dependence of precipitation (d).

\section{Discussion}

In this study, we present the first quantitative study of modes of seed formation in R. kuepferi over the entire distribution range in the European Alps. Additional to the geographical differentiation of cytotypes recorded previously (Cosendai and Hörandl 2010), we were able to demonstrate a positive correlation of apomictic mode of reproduction to elevation and related environmental factors. On average, apomictic tetraploids are found at higher elevations than sexual diploids. Hence, the niche shift to colder temperatures as reported by Kirchheimer et al. (2016) is mostly an effect of elevation (average decrease of $0.6^{\circ} \mathrm{C}$ per $100 \mathrm{~m}$; Nagy and Grabherr 2009). If the shift to colder temperature would be just a latitude effect (average decrease of temperature c. $0.75^{\circ} \mathrm{C}$ per degree), then the northernmost populations would have to occur at lower elevations (c. $300 \mathrm{~m}$ ) to keep their niche constant (e.g. many high alpine plants occur in the Arctic on sea level; Körner 2003). However, this is not the case in R. kuepferi. This niche shift to higher elevations would be also in line with the general trend of apomicts to occur in more extreme habitats compared to their diploid sexual progenitors, 
as postulated by the Frozen Niche Variation model. However, there is no niche expansion, as the amplitude was not expanded to lower elevations.

Large-scale studies of well-known examples that exhibit diverging distributions between sexvals and asexuals, such as the Crepis complex (Babcock and Stebbins 1938, Bierzychudek 1985), Paspalum simplex (Urbani et al. 2002), Taraxacum officinale agg. (Van Dijk 2003) and Crataegus (Lo et al. 2013) indicate classical patterns of Geographical Parthenogenesis, with polyploid apomicts occupying larger and colder areas than their diploid sexual relatives. Other examples in which apomicts do not possess an unequal distribution or even a smaller one compared to sexuals are Hieracium pilosella (Fehrer et al. 2007) and particularly the Boechera holboellii complex, with apomicts occurring more in southern parts of North America (Dobeš et al. 2004a, b) and an overall diffuse distribution pattern (Sharbel et al. 2005, Mau et al. 2015). However, in the latter two cases, the ploidy differentiation between modes of reproduction is not so pronounced, as sexuals in Hieracium pilosella are tetraploids (Mráz et al. 2009), while apomicts in Boechera include triploid and diploid taxa (Aliyu et al. 2010, Lovell et al. 2013). Hence, only a combination of polyploidy and asexuality versus diploid sexuality seems to establish a pattern of Geographical Parthenogenesis in plants (Hörandl 2006, Hörandl et al. 2008).

We found a surprisingly high diversity in the reproductive pathways in $R$. kuepferi, both on individual and on population level. A remarkably high proportion of tetraploid individuals formed seeds which are, to a varying degree, based on sexual pathways. Facultative apomixis is quite typical for plants reproducing via apospory because apomictic and sexual development run initially in parallel (Asker and Jerling 1992, Krahulcová et al. 2004, Dobeš et al. 2013, Klatt et al. 2016). Apospory was observed in R. kuepferi by Burnier et al. (2009). Generally, the mode of reproduction in $R$. kuepferi seems to be even more variable than expected. Indeed, most of the older studies on asexual reproduction in plants suggest that apomixis and polyploidy are almost exclusively and functionally correlated (Gustafsson 1946; Bierzychudek 1985, Carman 1997, Koltunow and Grossniklaus 2003). In contrast, our results provide evidence that this is not necessarily the case. Although most diploid individuals reproduce strictly sexual, a non-negligible amount of plants from three independent, geographically separated populations feature facultative apomixis (Fig. 1). Across numerous studied taxa, diploid plants expressing regularly functional apomictic seed formation in natural diploid populations are only known in the genus Boechera (Dobeš et al. 2006, Aliyu et al. 2010) and 
in various Paspalum species (Siena et al. 2008). Boechera has however a very complex taxonomic structure with more than 8o highly polymorphic species and hybrids (Koch et al. 2003, Dobeš et al. 2004a, b). Several studies found high levels of heterozygosity in diploid apomictic species of the complex, concluding them to be of hybrid origin (Beck et al. 2012, Dobeš et al. 2004a, b, Kiefer et al. 2009, Kiefer and Koch 2012, Schranz et al. 2005). A hybridogeneous origin as prerequisite for diploid apomixis cannot be completely ruled out in Boechera. In Ranunculus auricomus, diploid apomicts were observed just in experimentally produced dihaploid progenies, or in synthetic hybrids under garden conditions (Nogler 1984, Hojsgaard et al. 2014). Our quantitatively orientated study illustrates that large scale seed screenings are required to detect small but non-negligible amounts of facultative apomixis in diploids in natural populations, which would otherwise remain overlooked.

We found most asexually reproducing individuals at higher elevations under generally colder conditions. However, we cannot disentangle this phenomenon from ploidy effects as observed by Kirchheimer et al. (2016). Even among tetraploid populations at highest elevations, both sexual and apomictic seed formation occurs (Fig. 3b). A non-linear correlation of apomixis and elevation was also observed in subnivale plants in the highest zones of the Alps where sexuality is prevalent (Hörandl et al. 2011). The same phenomenon appears in high latitudes, as apomicts become rare in arctic and subantarctic floras (Asker and Jerling 1992). Furthermore, there is no geographical pattern in the degree of facultative sexuality over the whole distribution range of tetraploids (Fig. 1). The lack of a correlation of geographical distance to frequencies of apomictic reproduction does not support the idea of a prevalence of long distance dispersal for founding apomictic populations. Instead, we assume predominantly a fast stepwise dispersal aided by self-fertility and apomixis for the colonization of the Alps. This scenario is also supported by the lack of population genetic structure within the tetraploid range (Cosendai et al. 2013). Diploid populations with apomictic seed formation occur just in the diploid refugial areas, implying that apomixis alone is unsuccessful (Fig. 1). Tetraploid obligate sexual individuals were not observed in our study. Taken together, neither apomixis nor polyploidy alone explain the geographical pattern; only the combination of both factors is successful.

Focusing on the reproductive fitness of $R$. kuepferi, we found no positive effect of ploidy on the development of seeds. Seed set is overall significantly lower in polyploids than in diploids. However, diploids showed a significant negative correlation to elevation, and were in the 
highest populations between 2200 and $2400 \mathrm{~m}$ within the range of seed set of tetraploids (Fig. 3a). Seed set in diploids may be in highest elevations negatively influenced by pollinator limitation, or by a worse adaptation of reproductive tissues to cold temperatures (see below). Tetraploids, in contrast, remain more stable on a low level of seed set over the whole altitudinal gradient (Fig. 3a). Low seed set is probably a consequence of autopolyploidy. In sexual autopolyploids, profound difficulties may arise during chromosome segregation at meiosis due to the increased complexity of pairings among doubled and therefore identical chromosome sets, which mostly pair as multivalents. An efficient segregation of such multivalents in case of autotetraploid $R$. kuepferi remains difficult (Cosendai et al. 2011). Common segregation issues within enlarged chromosome sets can lead to disadvantageous irregularities such as aneuploidy (Birchler et al. 2007, Ozias-Akins and Van Dijk 2007). In polyploid R. kuepferi, we have discovered only very few cases of triploid embryos in the seed screening. We suppose that triploid megaspores resulting from disturbed meiosis largely abort during gametogenesis, which would explain the high proportions of aborted seeds (see also Cosendai and Hörandl 2010). High proportions of aborted pollen (Huber 1988) support this hypothesis. However, these developmental disturbances appear to be independent from elevation and associated environmental factors (Fig. 3a).

Nevertheless, despite a significantly worse overall reproductive fitness, tetraploid apomicts seem to be the only successful cytotype occupying the Alps as evident from the observed distribution patterns. One could argue this might result from implications of polyploidization itself. There is a long-lasting debate about the advantages or disadvantages of polyploidy and many hints are yet available which suggest a quite beneficial effect of genome doubling under certain circumstances (Ohno 1970, Comai 2005, Otto 2007, Soltis and Soltis 2009). Especially if polyploids manage to adapt, they possess the potential to establish efficient competitors to their diploid ancestors (Comai 2005). A multitude of studies suggest that possible success is mainly based on a potential manifold advantage of polyploidy. In polyploids, overall performance is often increased due to the heterosis effect (Birchler et al. 2010). According to some studies, putative autopolyploids even show stronger heterosis compared to allopolyploid hybrids (Bingham et al. 1994, Birchler et al. 2010).

However, the results of our morphological measurements in $R$. kuepferi were the contrary to expectations of heterosis effects: diploids proved to produce more leaves and flowers, exhibiting a seemingly higher vigour at least compared to the tetraploid plants, with a negative 
correlation to elevation. But only tetraploids occurred at higher elevations $>2400$ m.a.s.l., where the growing season starts later and a shorter vegetation period is available for growth. Moreover, low temperatures slow down cell cycle and cell differentiation processes (Körner 2003). In general, alpine plants produce fewer cells with a tendency to keep cell size constant, and as a result alpine plants often show a syndrome of 'alpine dwarfism' which is also adaptive to harsh climatic conditions at higher elevations (Körner 2003). Another potential limiting factor for successful plant reproduction is summer frost at high elevations, especially when reproductive tissues are not covered by snow and exposed to frost (Ladinig et al. 2013). In situations of summer frost, the taller growth and richer flower production of diploid $R$. kuepferi could be rather a disadvantage for successful seed production. Hence, tetraploid $R a-$ nunculus kuepferi probably adjusts its growth better to high alpine conditions. To which extent the observed morphological parameters are influenced by phenotypic plasticity or represent a heritable feature, needs to be studied.

Assets and drawbacks related to performance are not limited to hybrid vigour. Performance can also be positively influenced by direct consequences of present genome duplication. Polyploids maintain heterozygosity which can be important when isolated and severely bottlenecked, and autopolyploids preserve heterozygosity even better than hybrids (Comai 2005). In R. kuepferi, genetic diversity in tetraploid populations was as high as in diploids in all measures (Cosendai et al. 2013). Preserving genetic diversity and heterozygosity could have been helpful to escape the consequences resulting from inbreeding depression and genetic bottlenecks, as tetraploid apomictic individuals were spreading rapidly eastwards from the original core area in the south-western Alps.

Furthermore, gene duplication establishes the ability to diversify gene functions (Prince and Pickett 2002, Adams and Wendel 2005, Moore and Purugganan 2005). Sub- or even neofunctionalization of redundant genes possibly cause niche shifts (Lynch and Walsh 2007). Additionally, gene expression may be modified by the occurrence of doubled alleles (Wang et al. 2004, Hegarty et al. 2006, 2011). One should assume that expression patterns of most diploid sexual genotypes in a population are optimized to certain environmental conditions on site (Comai 2005). However, relevant differences in effects on transcriptomes were detected among allopolyploidy and mere genome doubling (Hegarty et al. 2005, 2006). Hegarty et al. 
(2006) further suggested that genome doubling has a 'calming effect' on hybridization-induced transcriptome shock. Thus, in autopolyploids, regulatory changes need not necessarily be unfavourable, but might allow for faster adaptation.

We hypothesize that a physiological ability to withstand conditions at high elevations is positively influenced by polyploidization in R. kuepferi. Aside from ranges, absolute elevations of tetraploid populations are significantly higher than in diploids. It is known that cell structures fundamentally change with the doubling of the genome, almost always resulting in an enlargement of cell size (Melaragno et al. 1993, Levin 2002). Compared to its alpine competitors, $R$. kuepferi is a fast developing plant, which is among the first sprouting and flowering after snow melting; after fruiting, the vegetative parts wither and disappear rapidly. Diploids generally bloom, fruit and ripe earlier than the tetraploids. Apart from climatic differences influencing growing seasons due to altitudinal ranges in the wild, this tendency is also apparent in cultivation when kept under same conditions (C. Schinkel and E. Hörandl, pers. obs.). An increase in cell size and a concordantly altered surface to volume ratio are known to be advantageous for cells with high metabolic rates (Comai 2005), and may allow a reduction of cell number (see above). Furthermore, alpine plants have to adapt their carbon uptake to lower CO 2 partial atmospheric pressure (Körner 2003). Taken together, the observed tendency to reduce growth in tetraploid $R$. kuepferi is probably an adaptation to the short vegetation period, to lower temperatures, and to lower carbon availability at higher elevations. Polyploidy has further pronounced effects on photosynthesis performance, e.g. increasing electron transport capacity (Coate et al. 2012), which might be advantageous under higher UV irradiation and light intensity in higher elevations. Other aspects of the observed niche shift of tetraploids, like a tendency to more acid soils (Kirchheimer et al. 2016), could also relate to polyploidy rather than to mode of reproduction.

Apomictic development, in turn, may help to accelerate seed formation under conditions of shorter vegetation periods. Diploids apparently lost their fitness advantage in higher elevations. We could not observe differences in timing of female gametophyte development between sexual and apomictic pathways (C. Schinkel, unpubl. data); but embryogenesis and seed development could be accelerated, as cross-fertilization and pollinator visits are not needed for tetraploids. Previous experimental work has shown that tetraploids are self-fertile whereas diploids are largely self-incompatible (Cosendai et al. 2013). Hence, reduced seed set in diploid sexuals at highest elevations could be due to pollinator limitation (Arroyo et al. 
1982, Körner 2003). In contrast, apomictic colonizers do not experience pollinator and mate limitation, which are important aspects of Baker's law (Pannell et al. 2015).

In this study, we primarily focused on colder temperatures which prevail at higher elevations and may directly influence reproduction. De Storme et al. (2012) found that short periods of cold stress are able to induce the production of a certain amount of polyploid pollen in diploid Arabidopsis thaliana. According to our FCSS data, several asexual reproduction pathways could theoretically involve unreduced pollen, mainly found in tetraploids (Table 1). However, the male contribution to endosperm DNA content by unreduced pollen overlaps in most pathways with that of a possible double fertilization of the endosperm with both sperm nuclei of reduced pollen. Based only on the data obtained by the FCSS, one can only ascertain a few cases of unreduced pollen (pathway $\mathrm{B}_{5}$, Table 1 ). On the female side, unreduced egg cells were formed in all apomictic pathways and in BIII hybrids, and occurred in all cytotypes. Experimental cold treatments triggered spontaneous apomixis in diploids, suggesting a direct influence of temperature on female gamete formation (S. Klatt, unpubl. data). Whether all of these cases are due to the development of somatic cells (apospory) as reported by Burnier et al. (2009) or also to unreduced megaspores formed by restitutional meiosis (diplospory), needs to be studied. Moreover, we analyzed wild populations and used climate data of relatively low resolution. However, environmental parameters in the Alps may change on small scales (Kirchheimer et al. 2016). Thus, obtained results do not account for any microclimatic variation among sample sites. Whether there is a direct influence of cold on polyploidization in $R$. kuepferi or not, remains an open question at present to be addressed.

\section{Conclusions}

In summary, the major outcome of this study underpins the strong correlation of ploidy with elevation and correlated climatic variables. Conversely, the mode of reproduction turned out to be of substantial variance independent of cytotypes and indeed is also correlated to elevation, but cannot be disentangled from polyploidy. A combination effect, postulated by Hörandl (2006), likely applies to R. kuepferi. Positive effects of polyploidy are probably morphological and physiological adaptations to conditions at high elevations, enabling them to conduct a significant niche shift in the allopatric range (Kirchheimer et al. 2016). Positive effects of apomixis are probably increasing the capacity for a rapid range expansion by founding populations with single diaspores without mate and pollinator limitation following 
Baker's law (Pannell et al. 2015). In higher elevations, apomixis may help to shorten developmental pathways to seed formation which is an advantage in short vegetation periods. Facultative sexuality, in turn, preserves genetic diversity and adaptive potential in small founder populations (Cosendai et al. 2013). 


\section{References}

Adams KL and Wendel JF. 2005. Polyploidy and genome evolution in plants. Current Opinion in Plant Biology 8:135-141

Aliyu OM, Schranz ME and Sharbel TF. 2010. Quantitative variation for apomictic reproduction in the genus Boechera (Brassicaceae). American Journal of Botany 97:1719-1731

Arroyo MTK, Primack R and Armesto J. 1982. Community studies in pollination ecology in the high temperate Andes of Central Chile. I. Pollination mechanisms and altitudinal variation. American Journal of Botany 69:82-97

Asker SE and Jerling L. 1992. Apomixis in Plants. CRC Press, Boca Raton

Babcock EB and Stebbins GL. 1938. The American species of Crepis: their interrelationships and distribution as affected by polyploidy and apomixis. Publications of the Carnegie Institution of Washington

Baker HG. 1955. Self-compatibility and establishment after "long-distance" dispersal. Evolution 9:347-349

Baker HG. 1965. Characteristics and modes of origin of weeds. In: Baker HG (eds) The genetics of colonizing species. Academic Press, New York, pp 147-172

Baker HG. 1967. Support for Baker's Law-as a rule. Evolution 21:853-856

Beck JB, Alexander PJ, Allphin L, Al-Shehbaz IA, Rushworth C, Bailey CD and Windham MD. 2012. Does hybridization drive the transition to asexuality in diploid Boechera?. Evolution 66:985-995

Bell G. 1982. The Masterpiece of Nature: The Evolution and Genetics of Sexuality. Cambridge University Press Archive, Cambridge

Bierzychudek P. 1985. Patterns in plant parthenogenesis. Experientia 41:1255-1264

Bingham ET, Groose RW, Woodfield DR and Kidwell KK. 1994. Complementary gene interactions in alfalfa are greater in autotetraploids than diploids. Crop Science 34:823-829

Birchler JA, Yao H and Chudalayandi S. 2007. Biological consequences of dosage dependent gene regulatory systems. Biochimica et Biophysica Acta (BBA) - Gene Structure and Expression 1769:422-428

Birchler JA, Yao H, Chudalayandi S, Vaiman D and Veitia RA. 2010. Heterosis. The Plant Cell 22:21052112

Bomblies K, Higgins JD and Yant L. 2015. Meiosis evolves: adaptation to external and internal environments. New Phytologist 208:306-323

Burnier J, Buerki S, Arrigo N, Küpfer P and Alvarez N. 2009. Genetic structure and evolution of Alpine polyploid complexes: Ranunculus kuepferi (Ranunculaceae) as a case study. Molecular Ecology 18:3730-3744

Carman JG. 1997. Asynchronous expression of duplicate genes in angiosperms may cause apomixis, bispory, tetraspory, and polyembryony. Biological Journal of the Linnean Society 61:51-94

Coate JE, Luciano AK, Seralathan V, Minchew KJ, Owens TG and Doyle JJ. 2012. Anatomical, biochemical, and photosynthetic responses to recent autopolyploidy in Glycine dolichocarpa (Fabaceae). American Journal of Botany 99:55-67

Comai L. 2005. The advantages and disadvantages of being polyploid. Nature Reviews Genetics 6:836-846 
Cosendai A-C and Hörandl E. 2010. Cytotype stability, facultative apomixis and Geographical Parthenogenesis in Ranunculus kuepferi (Ranunculaceae). Annals of Botany 105:457-470

Cosendai A-C, Rodewald J and HörandI E. 2011. Origin and distribution of autopolyploids via apomixis in the alpine species Ranunculus kuepferi (Ranunculaceae). Taxon 60:355-364

Cosendai A-C, Wagner J, Ladinig U, Rosche C and Hörandl E. 2013. Geographical parthenogenesis and population genetic structure in the alpine species Ranunculus kuepferi (Ranunculaceae). Heredity 110:560-569

De Storme N, Copenhaver GP and Geelen D. 2012. Production of diploid male gametes in Arabidopsis by cold-induced destabilization of postmeiotic radial microtubule arrays. Plant Physiology 160:18081826

De Storme N, Zamariola L, Mau M, Sharbel TF and Geelen D. 2013. Volume-based pollen size analysis: an advanced method to assess somatic and gametophytic ploidy in flowering plants. Plant Reproduction 26:65-81

Dobeš C, Koch M and Sharbel TF. 2006. Embryology, karyology, and modes of reproduction in the north american genus Boechera (Brassicaceae): a compilation of seven decades of research. Annals of the Missouri Botanical Garden 93:517-534

Dobeš C, Lückl A, Hülber K and Paule J. 2013. Prospects and limits of the flow cytometric seed screen insights from Potentilla sensu lato (Potentilleae, Rosaceae). New Phytologist 198:605-616

Dobeš C, Mitchell-Olds T and Koch M. 2004. Intraspecific diversification in North American Boechera stricta (= Arabis drummondii), Boechera $x$ divaricarpa, and Boechera holboellii (Brassicaceae) inferred from nuclear and chloroplast molecular markers - an integrative approach. American Journal of Botany 91:2087-2101

Dobeš C, Mitchell-Olds T and Koch M. 2004. Extensive chloroplast haplotype variation indicates Pleistocene hybridization and radiation of North American Arabis drummondii, A. x divaricarpa, and $A$. holboellii (Brassicaceae). Molecular Ecology 13:349-370

Doležel J, Greilhuber J and Suda J. 2007. Estimation of nuclear DNA content in plants using flow cytometry. Nature Protocols 2:2233-2244

Fehrer J, Krahulcová A, Krahulec F, Chrtek J, Rosenbaumová R and Bräutigam S. 2007. Evolutionary aspects in Hieracium subgenus Pilosella. In: Hörandl E, Grossniklaus U, van Dijk P, Sharbel T (eds) Apomixis: Evolution, Mechanisms and Perspectives In: Hörandl E, Grossniklaus U, van Dijk P, Sharbel T (eds) Apomixis: Evolution, Mechanisms and Perspectives. Gantner, Ruggell, pp 359-395

Fox J. 2005. The R Commander: A basic-statistics graphical user interface to R. Journal of Statistical Software 14:1-42

Greilhuber J, Doležel J, Lysák MA and Bennett MD. 2005. The origin, evolution and proposed stabilization of the terms 'Genome Size' and 'C-Value' to describe nuclear DNA contents. Annals of Botany $95: 255-260$

Gustafsson A. 1946. Apomixis in higher plants. Lunds Universitets Årsskrift NF I/ 42(3):1-67 
Hegarty MJ and Hiscock SJ. 2008. Genomic clues to the evolutionary success of polyploid plants. Current Biology 18:435-444

Hegarty MJ, Barker GL, Wilson ID, Abbott RJ, Edwards KJ and Hiscock SJ. 2006. Transcriptome shock after interspecific hybridization in Senecio is ameliorated by genome duplication. Current Biology 16:1652-1659

Hegarty MJ, Batstone T, Barker GL, Edwards KJ, Abbott RJ and Hiscock SJ. 2011. Nonadditive changes to cytosine methylation as a consequence of hybridization and genome duplication in Senecio (Asteraceae). Molecular Ecology 20:105-113

Hegarty MJ, Jones JM, Wilson ID, Barker GL, Coghill JA, Sanchez-Baracaldo P, Liu G, Buggs RJA, Abbott RJ, Edwards KJ and Hiscock SJ. 2005. Development of anonymous CDNA microarrays to study changes to the Senecio floral transcriptome during hybrid speciation. Molecular Ecology 14:2493-2510

Hijmans RJ, Cameron SE, Parra JL, Jones PG and Jarvis A. 2005. Very high resolution interpolated climate surfaces for global land areas. International Journal of Climatology 25:1965-1978

Hojsgaard D, Greilhuber J, Pellino M, Paun O, Sharbel TF and Hörandl E. 2014. Emergence of apospory and bypass of meiosis via apomixis after sexual hybridisation and polyploidisation. New Phytologist 204:1000-1012

Hörandl E. 2006. The complex causality of Geographical Parthenogenesis. New Phytologist 171(3):525-538

Hörandl E. 2009. Geographical parthenogenesis: opportunities for asexuality. In: Schön I, Martens K, Van Dijk P (eds) Lost Sex. Springer, Heidelberg, pp 161-186

Hörandl E. 2010. The evolution of self-fertility in apomictic plants. Sexual Plant Reproduction 23:73-86

Hörand IE, Cosendai A-C and Temsch EM. 2008. Understanding the geographic distributions of apomictic plants: a case for a pluralistic approach. Plant Ecology and Diversity 1:309-320

Hörandl E, Dobeš C, Suda J, Vit P, Urfus T, Temsch EM, Cosendai A-C, Wagner J and Ladinig U. 2011. Apomixis is not prevalent in subnival to nival plants of the European Alps. Annals of Botany 108:381-390

Huber W. 1988. Natürliche Bastardierungen zwischen weissblühenden Ranunculus-Arten in den Alpen [Natural hybridizations between white-flowered species of Ranunculus in the Alps]. Veröffentlichungen des Geobotanischen Institutes der Eidgenössischen Technischen Hochschule, Stiftung Rübel, Zürich 100:1-160

Kearney M. 2005. Hybridization, glaciation and Geographical Parthenogenesis. Trends in Ecology \& Evolution 20:495-502

Kiefer C and Koch MA. 2012. A Continental-Wide Perspective: The gene pool of nuclear encoded ribosomal DNA and single-copy gene sequences in North American Boechera (Brassicaceae). PLOS ONE $7(5): e 36491$ 
Kiefer C, Dobes C, Sharbel TF and Koch MA. 2009. Phylogeographic structure of the chloroplast DNA gene pool in North American Boechera - A genus and continental-wide perspective. Molecular Phylogenetics and Evolution 52:303-311

Kirchheimer B, Schinkel CCF, Dellinger AS, Klatt S, Moser D, Winkler M, Lenoir J, Caccianiga M, Guisan A, Nieto-Lugilde D, Svenning J-C, Thuiller W, Vittoz P, Willner W, Zimmermann NE, Hörandl E and Dullinger S. 2016. A matter of scale: apparent niche differentiation of diploid and tetraploid plants may depend on extent and grain of analysis. Journal of Biogeography 43:716-726

Klatt S, Hadacek F, Hodač L, Brinkmann G, Eilerts M, Hojsgaard D and Hörandl E. 2016. Photoperiod extension enhances sexual megaspore formation and triggers metabolic reprogramming in facultative apomictic Ranunculus auricomus. Frontiers in Plant Science 7:278-293

Koch M, Dobeš C and Mitchell-Olds T. 2003. Multiple hybrid formation in natural populations: concerted evolution of the internal transcribed spacer of nuclear ribosomal DNA ITS in North American Arabis divaricarpa (Brassicaceae). Molecular Biology and Evolution 20:338-350

Koltunow AM and Grossniklaus U. 2003. Apomixis: a developmental perspective. Annual Review of Plant Biology 54:547-574

Körner C. 2003. Alpine plant life: functional plant ecology of high mountain ecosystems; with 47 tables. Springer, Berlin

Krahulcová A, Papouskuvá S and Krahulec F. 2004. Reproduction mode in the allopolyploid facultatively apomictic hawkweed Hieracium rubrum (Asteraceae, H. subgen. Pilosella). Hereditas 141:19-30

Kudo $\mathrm{G}$ and Hirao AS. 2005. Habitat-specific responses in the flowering phenology and seed set of alpine plants to climate variation: implications for global-change impacts. Population Ecology 48:49-58

Ladinig U, Hacker J, Neuner G and Wagner J. 2013. How endangered is sexual reproduction of highmountain plants by summer frosts? Frost resistance, frequency of frost events and risk assessment. Oecologia 171:743-760

Levin DA. 2002. The role of chromosomal change in plant evolution. Oxford University Press, Oxford

Lo EYY, Stefanović S and Dickinson TA. 2013. Geographical parthenogenesis in Pacific Northwest hawthorns (Crataegus; Rosaceae). Botany 91:107-116

Lovell JT, Aliyu OM, Mau M, Schranz ME, Koch M, Kiefer C, Song B-H, Mitchell-Olds T and Sharbel TF. 2013. On the origin and evolution of apomixis in Boechera. Plant Reproduction 26:309-315

Lynch M and Walsh B. 2007. The origins of genome architecture. Sinaver Associates, Sunderland

Matzk F, Meister A and Schubert I. 2000. An efficient screen for reproductive pathways using mature seeds of monocots and dicots. The Plant Journal 21:97-108

Mau M, Lovell JT, Corral JM, Kiefer C, Koch M, Aliyu OM and Sharbel TF. 2015. Hybrid apomicts trapped in the ecological niches of their sexual ancestors. Proceedings of the National Academy of Sciences of the United States of America. 112:2357-2365

Melaragno JE, Mehrotra B and Coleman AW. 1993. Relationship between endopolyploidy and cell size in epidermal tissue of Arabidopsis. The Plant Cell 5:1661-1668 
Mogie M and Ford H. 1988. Sexual and asexual Taraxacum species. Biological Journal of the Linnean Society $35: 155-168$

Moore RC and Purugganan MD. 2005. The evolutionary dynamics of plant duplicate genes. Current Opinion in Plant Biology 8:122-128

Mráz P, Šingliarová P, Urfus T and Krahulec F. 2009. Cytogeography of Pilosella officinarum (Compositae): Altitudinal and longitudinal differences in ploidy level distribution in the Czech Republic and Slovakia and the general pattern in Europe. Annals of Botany 101:59-71

Nagy L and Grabherr G. 2009. The biology of alpine habitats. Oxford University Press, New York

Nicotra AB, Atkin OK, Bonser SP, Davidson AM, Finnegan EJ, Mathesius U, Poot P, Purugganan MD, Richards CL, Valladares F and Van Kleunen M. 2010. Plant phenotypic plasticity in a changing climate. Trends in Plant Science 15:684-692

Nogler GA. 1984. Gametophytic apomixis. In: Johri PBM (eds) Embryology of angiosperms. Springer, Berlin, $p p$ 475-518

Ohno S. 1970. Evolution by gene duplication. Springer, Berlin

Osborn TC, Pires CJ, Birchler JA, Auger DL, Chen JZ, Lee H-S, Comai L, Madlung A, Doerge RW, Colot V and Martienssen RA. 2003. Understanding mechanisms of novel gene expression in polyploids. Trends in Genetics 19:141-147

Otto SP. 2007. The evolutionary consequences of polyploidy. Cell 131:452-462

Ozias-Akins P and Van Dijk PJ. 2007. Mendelian genetics of apomixis in plants. Annual Review of Genetics 41:509-537

Pannell JR, Auld JR, Brandvain Y, Burd M, Busch JW, Cheptou PO, Conner JK, Goldberg EE, Grant AG, Grossenbacher DL, Hovick SM, Igic B, Kalisz S, Petanidou T, Randle AM, De Casas RR, Pauw A, Vamosi JC and Winn AA. 2015. The scope of Baker's law. New Phytologist 208:656-667

Prince VE and Pickett FB. 2002. Splitting pairs: the diverging fates of duplicated genes. Nature Reviews Genetics 3:827-837

R Core Team. 2014. R: A language and environment for statistical computing. $R$ Foundation for Statistical Computing

Ramsey J and Schemske DW. 1998. Pathways, mechanisms, and rates of polyploid formation in flowering plants. Annual Review of Ecology and Systematics 29:467-501

Sarkar D. 2008. Lattice: multivariate data visualization with R. Springer, Berlin

Schranz ME, Dobeš C, Koch MA and Mitchell-Olds T. 2005. Sexual reproduction, hybridization, apomixis, and polyploidization in the genus Boechera (Brassicaceae). American Journal of Botany 92:17971810

Sharbel TF, Mitchell-Olds TM, Dobes C, Kantama L and de Jong H. 2005. Biogeographic distribution of polyploidy and B chromosomes in the apomictic Boechera holboellii complex. Cytogenetic and Genome Research 109:283-292 
Siena LA, Sartor ME, Espinoza F, Quarin CL and Ortiz JPA. 2008. Genetic and embryological evidences of apomixis at the diploid level in Paspalum rufum support recurrent auto-polyploidization in the species. Sexual Plant Reproduction 21:205-215

Soltis DE and Soltis PS. 2009. The role of hybridization in plant speciation. Annual Review of Plant Biology $60: 561-588$

Talent N and Dickinson TA. 2007. Endosperm formation in aposporous Crataegus (Rosaceae, Spiraeoideae, tribe Pyraceae): parallels to Ranunculaceae and Poaceae. New Phytologist 173:231-249

Urbani MH, Quarín CL, Espinoza F, Penteado MIO and Rodrigues IF. 2002. Cytogeography and reproduction of the Paspalum simplex polyploid complex. Plant Systematics and Evolution 236:99-105

Van Dijk PJ. 2003. Ecological and evolutionary opportunities of apomixis: insights from Taraxacum and Chondrilla. Philosophical Transactions of The Royal Society B: Biological Sciences 358:1113-1121

Vandel A. 1928. La parthenogenese geographique: Contribution a l'etude biologique et cytologique de la parthenogenese naturelle. Bulletin biologique de la France et de la Belgique 62:164-281

Vrijenhoek RC. 1984. Ecological differentiation among clones: The Frozen Niche Variation model. In: Wöhrmann K, Loeschcke V (eds) Population Biology and Evolution. Springer, Berlin, pp 217-231

Vrijenhoek RC. 1994. Unisexual fish: Model systems for studying ecology and evolution. Annual Review of Ecology and Systematics 25:71-96

Vrijenhoek RC and Parker ED Jr. 2009. Geographical Parthenogenesis: General Purpose Genotypes and Frozen Niche Variation. In: Schön I, Martens K and Van Dijk PJ (eds) Lost Sex. Springer, Dordrecht, pp 99-131

Wagner J and Reichenegger B. 1997. Phenology and seed development of the Alpine sedges Carex curvula and Carex firma in response to contrasting topoclimates. Arctic, Antarctic, and Alpine Research 29:291-299

Wagner J and Mitterhofer E. 1998. Phenology, seed development, and reproductive success of an Alpine population of Gentianella germanica in climatically varying years. Botanica Acta 111:159-166

Wang J, Tian L, Madlung A, Lee H-S, Chen M, Lee JJ, Watson B, Kagochi T, Comai L and Chen ZJ. 2004. Stochastic and epigenetic changes of gene expression in Arabidopsis polyploids. Genetics 167:1961-1973 


\section{Supporting Information}
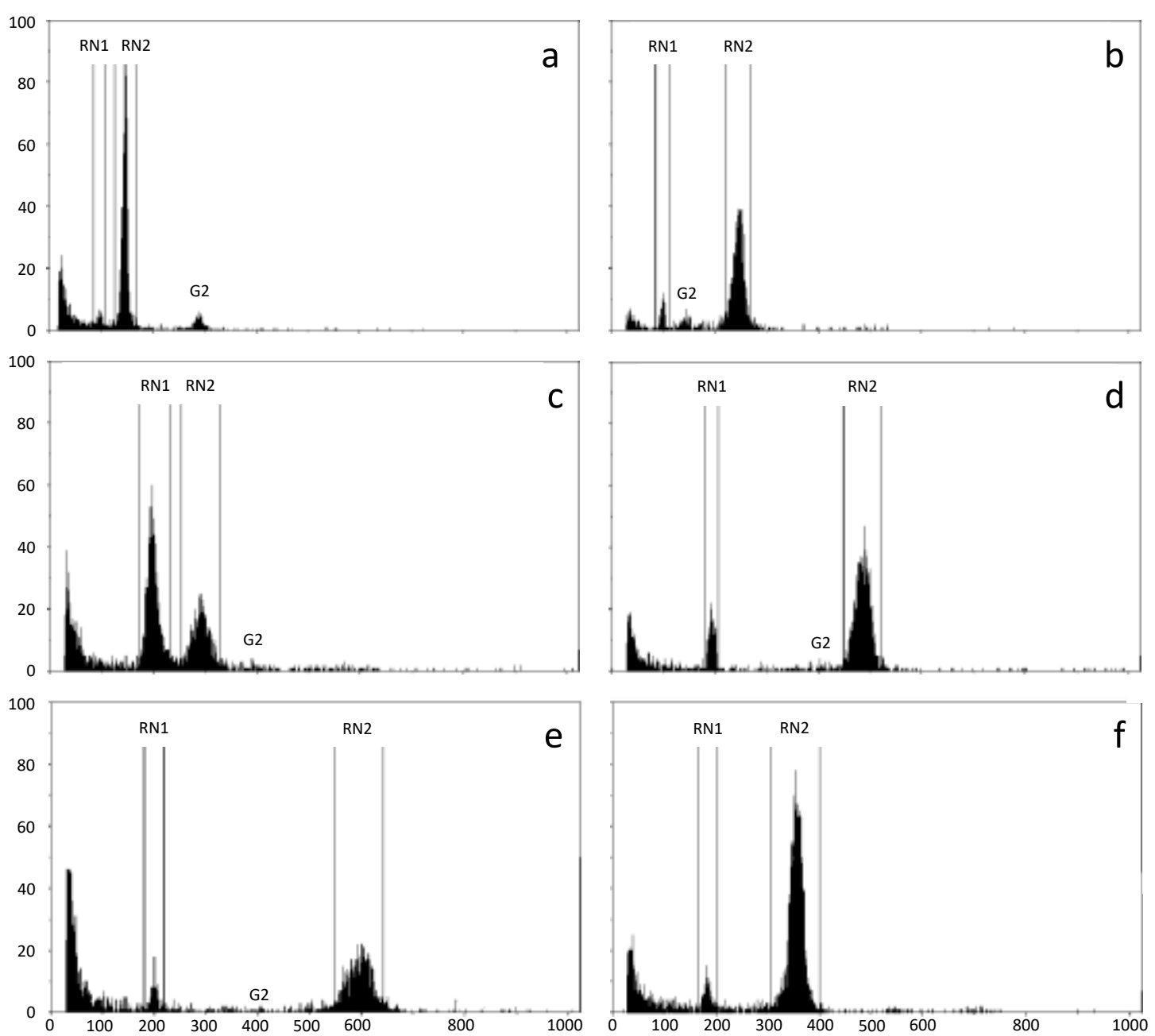

Figure S1. Flow histograms of the most frequent pathways (see Table 1). RN1, embryo peak; RN2, endosperm peak. a: Sexual seed formation in diploids (pathway A); b: Apomictic seed formation in diploids (pathway A2); c: Sexual seed formation in tetraploids (pathway C); d: Apomictic seed formation in tetraploids (pathway $C_{5}$ ).

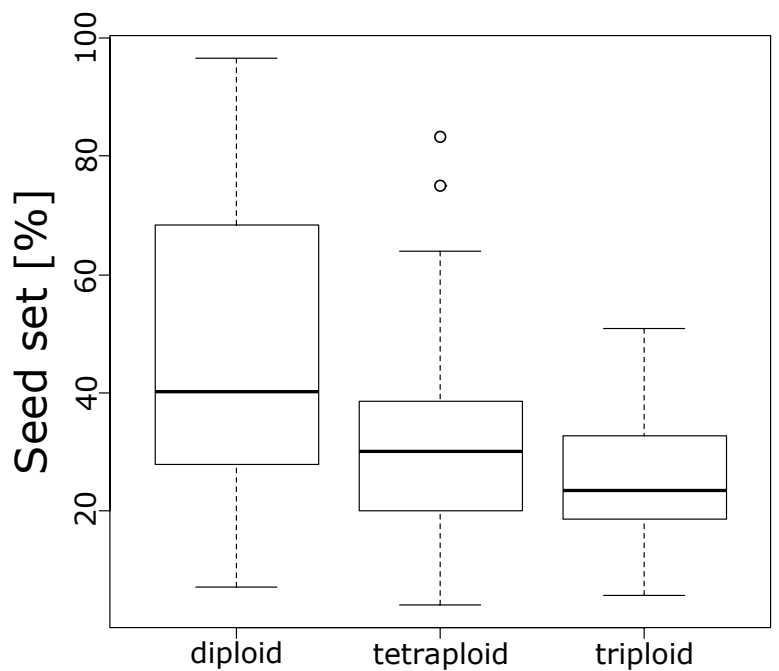

Figure S2. Boxplots of seed set between Ranunculus kuepferi cytotypes, based on percentage of well-developed achenes per flower, averaged per plant. Outliers are presented as black circles (o). 
Table S1. Table of sampled populations with geographical reference. pop = population number; $\mathrm{A}=\mathrm{Austria}, \mathrm{CH}$ = Switzerland, F = France, I = Italy, north and east denote coordinates; alt = altitude in $\mathrm{m}$ a.s.I.

\begin{tabular}{|c|c|c|c|c|c|}
\hline pop & country & region & north & east & alt \\
\hline 1 & $\mathrm{~F}$ & Provence-Alpes-Côte d'Azur & $43 \cdot 99972$ & 7.43028 & 2057 \\
\hline 2 & $\mathrm{~F}$ & Provence-Alpes-Côte d'Azur & 44.09583 & $7 \cdot 35950$ & 1873 \\
\hline 3 & $\mathrm{~F}$ & Provence-Alpes-Côte d'Azur & 44.20028 & 7.15639 & 2291 \\
\hline 4 & $\mathrm{~F}$ & Provence-Alpes-Côte d'Azur & 44.17528 & 6.89806 & 2050 \\
\hline 13 & $\mathrm{~F}$ & Rhônes-Alpes & $44 \cdot 54694$ & 6.70361 & 2059 \\
\hline 14 & $\mathrm{~F}$ & Provence-Alpes-Côte d'Azur & $44 \cdot 35489$ & $6.5055^{8}$ & 1880 \\
\hline 16 & $\mathrm{~F}$ & Provence-Alpes-Côte d'Azur & 45.03483 & 6.40242 & 2078 \\
\hline 17 & $\mathrm{~F}$ & Provence-Alpes-Côte d'Azur & 45.05069 & 6.39078 & 2357 \\
\hline 20 & $\mathrm{CH}$ & Valais & 46.34719 & 7.72411 & 2200 \\
\hline 23 & $\mathrm{~F}$ & Provence-Alpes-Côte d'Azur & 43.74931 & 6.65744 & 1616 \\
\hline 24 & $\mathrm{~F}$ & Provence-Alpes-Côte d'Azur & 44.15083 & 6.54361 & 1925 \\
\hline 25 & $\mathrm{~F}$ & Provence-Alpes-Côte d'Azur & $44 \cdot 90128$ & $5 \cdot 47617$ & 1435 \\
\hline 26 & $\mathrm{~F}$ & Provence-Alpes-Côte d'Azur & $44 \cdot 90033$ & 5.46900 & 1456 \\
\hline 27 & $\mathrm{~F}$ & Rhônes-Alpes & 44.83933 & $5 \cdot 42422$ & 1449 \\
\hline 28 & 1 & Piemonte & 44.24414 & 7.62906 & 1685 \\
\hline 29 & I & Piemonte & 44.23111 & 7.61722 & 2020 \\
\hline 30 & I & Piemonte & $44.1845^{8}$ & 7.62700 & 1743 \\
\hline 31 & I & Piemonte & 44.19522 & 7.65922 & 1937 \\
\hline 32 & 1 & Piemonte & 44.20667 & 7.14750 & 2320 \\
\hline 33 & I & Piemonte & 44.21305 & 7.14667 & 2328 \\
\hline 34 & $\mathrm{~F}$ & Rhônes-Alpes & 45.24244 & 6.95189 & 2120 \\
\hline 36 & $\mathrm{~F}$ & Rhônes-Alpes & $45 \cdot 38542$ & 7.04372 & 2152 \\
\hline 37 & I & Val d'Aosta & $45 \cdot 61622$ & $7 \cdot 55261$ & 2115 \\
\hline 38 & $\mathrm{~F}$ & Rhônes-Alpes & 45.67822 & 6.87800 & 2182 \\
\hline 40 & $\mathrm{CH}$ & Valais & 46.08250 & 7.01250 & 1860 \\
\hline 41 & 1 & Val d'Aosta & $45 \cdot 93195$ & 7.63944 & 2174 \\
\hline 42 & $\mathrm{CH}$ & Valais & 46.43300 & 7.86492 & 1789 \\
\hline 43 & $\mathrm{CH}$ & Valais & 46.25086 & 8.01800 & 2012 \\
\hline 45 & $\mathrm{CH}$ & Valais & 46.57119 & 8.41056 & 2400 \\
\hline 46 & $\mathrm{CH}$ & Tessin & 46.56367 & 8.79842 & 1937 \\
\hline 47 & $\mathrm{CH}$ & Graubunden & 46.54628 & 9.21139 & 2211 \\
\hline 48 & $\mathrm{CH}$ & Graubunden & 46.47153 & 9.72889 & 2262 \\
\hline
\end{tabular}




\begin{tabular}{|c|c|c|c|c|c|}
\hline 49 & $\mathrm{CH}$ & Graubunden & 46.58200 & 9.83672 & 2306 \\
\hline 50 & $\mathrm{CH}$ & Graubunden & 46.41275 & 10.02408 & 2322 \\
\hline 51 & $A$ & Tyrol & 47.14756 & 10.24817 & 2286 \\
\hline 53 & $\mathrm{CH}$ & Graubunden & 46.54856 & 10.43431 & 2456 \\
\hline 54 & I & Lombardia & 46.27239 & 10.57506 & 2303 \\
\hline 55 & $A$ & Tyrol & 46.87197 & 10.70836 & 2557 \\
\hline 57 & $A$ & Tyrol & 47.08960 & 11.54784 & 2313 \\
\hline 58 & I & Trentino Alto Adige/ Südtirol & 46.45667 & 11.88814 & 2117 \\
\hline 59 & 1 & Trentino Alto Adige/ Südtirol & 46.66434 & 12.18316 & 2391 \\
\hline 66 & I & Trentino Alto Adige/ Südtirol & 46.95562 & 11.51737 & 2101 \\
\hline 69 & I & Trentino Alto Adige/ Südtirol & 46.47751 & 11.81474 & 2297 \\
\hline 73 & $A$ & Tyrol & 47.21906 & 10.31961 & 2180 \\
\hline 74 & $A$ & Osttirol & 46.69904 & 12.44026 & 2117 \\
\hline 75 & $\mathrm{CH}$ & Graubunden & 46.52845 & 9.81119 & 2678 \\
\hline 77 & $\mathrm{CH}$ & Valais & 46.36692 & 7.65278 & 2259 \\
\hline 78 & $\mathrm{CH}$ & Valais & 46.22506 & 7.15989 & 2000 \\
\hline 79 & $\mathrm{CH}$ & Graubunden & 46.98753 & 10.35919 & 2280 \\
\hline 80 & $\mathrm{CH}$ & Graubunden & 46.66845 & 8.69997 & 2212 \\
\hline 81 & $A$ & Tyrol & 46.98753 & 10.32303 & 2526 \\
\hline 82 & I & Lombardia & 46.53867 & 10.43525 & 2500 \\
\hline 83 & $A$ & Tyrol & 47.04070 & 12.69105 & 2271 \\
\hline 84 & $A$ & Carinthia & 47.06989 & 12.84618 & 2236 \\
\hline 85 & A & Carinthia & 47.05495 & 12.86219 & 2184 \\
\hline 88 & $\mathrm{CH}$ & Graubunden & 46.51597 & 9.82125 & 2300 \\
\hline 89 & $\mathrm{CH}$ & Graubunden & 46.65067 & 9.75561 & 2265 \\
\hline 90 & $\mathrm{CH}$ & Valais & 46.12433 & $7 \cdot 51753$ & 2477 \\
\hline 91 & $\mathrm{CH}$ & Valais & 46.56295 & $8.3475^{8}$ & 2177 \\
\hline 92 & $\mathrm{CH}$ & Graubunden & 46.42364 & 9.63836 & 2260 \\
\hline 93 & $\mathrm{CH}$ & Valais & 46.20853 & $7 \cdot 72417$ & 2405 \\
\hline 94 & A & Tyrol & 46.99483 & 10.20550 & 2252 \\
\hline 96 & $\mathrm{~F}$ & Provence-Alpes-Côte d'Azur & 44.81775 & 6.73211 & 2300 \\
\hline 98 & I & Trentino Alto Adige/ Südtirol & 46.48304 & 12.05303 & 2233 \\
\hline 103 & I & Lombardia & 46.49211 & 10.20758 & 2290 \\
\hline 104 & I & Lombardia & 46.45142 & $10.3015^{8}$ & 2298 \\
\hline 106 & I & Trentino Alto Adige/ Südtirol & 46.94659 & 11.83144 & 2142 \\
\hline
\end{tabular}




\begin{tabular}{|c|c|c|c|c|c|}
\hline 107 & I & Trentino Alto Adige/ Südtirol & 46.51955 & 10.57536 & 2345 \\
\hline 108 & $\mathrm{CH}$ & Graubunden & 46.45392 & 9.98694 & 2171 \\
\hline 110 & I & Trentino Alto Adige/ Südtirol & 46.99229 & 12.11355 & 2312 \\
\hline 111 & $\mathrm{~F}$ & Provence-Alpes-Côte d'Azur & 44.27900 & 6.71922 & 2243 \\
\hline 112 & $\mathrm{~F}$ & Provence-Alpes-Côte d'Azur & 43.85250 & 6.35278 & 1626 \\
\hline 113 & $\mathrm{CH}$ & Graubunden & 46.64370 & 9.86642 & 2446 \\
\hline 114 & $\mathrm{~F}$ & Provence-Alpes-Côte d'Azur & 44.72089 & 6.91986 & 2339 \\
\hline 115 & $\mathrm{~F}$ & Provence-Alpes-Côte d'Azur & 44.24500 & 6.75611 & 1891 \\
\hline 116 & $\mathrm{~F}$ & Provence-Alpes-Côte d'Azur & 44.24797 & 6.76194 & 1953 \\
\hline 117 & $\mathrm{~F}$ & Provence-Alpes-Côte d'Azur & 43.74556 & 6.65583 & 1632 \\
\hline 118 & I & Piemonte & 44.23161 & 7.62833 & 1636 \\
\hline 119 & I & Piemonte & 44.26778 & 7.21111 & 1820 \\
\hline 120 & I & Piemonte & 44.22333 & $7.1205^{8}$ & 1966 \\
\hline 121 & $\mathrm{~F}$ & Provence-Alpes-Côte d'Azur & 44.20639 & 7.11556 & 1710 \\
\hline 200 & I & Piemonte & 44.18431 & 7.60347 & 1390 \\
\hline 201 & $\mathrm{~F}$ & Provence-Alpes-Côte d'Azur & 44.08044 & 6.63750 & 2025 \\
\hline 202 & $\mathrm{~F}$ & Provence-Alpes-Côte d'Azur & 44.15900 & 6.71461 & 1829 \\
\hline 203 & $\mathrm{~F}$ & Provence-Alpes-Côte d'Azur & 44.16294 & 6.71081 & 1840 \\
\hline 204 & $\mathrm{~F}$ & Provence-Alpes-Côte d'Azur & 44.15725 & 6.72920 & 1640 \\
\hline 205 & $\mathrm{~F}$ & Provence-Alpes-Côte d'Azur & 44.23711 & 6.70478 & 2265 \\
\hline 206 & $\mathrm{~F}$ & Provence-Alpes-Côte d'Azur & 44.24680 & 6.69880 & 2049 \\
\hline 207 & $\mathrm{~F}$ & Provence-Alpes-Côte d'Azur & $44 \cdot 30108$ & 6.56739 & 1921 \\
\hline 208 & $\mathrm{~F}$ & Provence-Alpes-Côte d'Azur & $44 \cdot 30244$ & 6.56097 & 1924 \\
\hline 209 & $\mathrm{~F}$ & Provence-Alpes-Côte d'Azur & 44.28967 & 6.74344 & 1942 \\
\hline 210 & $\mathrm{~F}$ & Provence-Alpes-Côte d'Azur & $44 \cdot 36180$ & 6.78758 & 2060 \\
\hline 211 & $\mathrm{~F}$ & Provence-Alpes-Côte d'Azur & $44 \cdot 32505$ & 6.85761 & 1983 \\
\hline 212 & $\mathrm{~F}$ & Provence-Alpes-Côte d'Azur & $44 \cdot 33314$ & 6.87167 & $225^{8}$ \\
\hline 213 & $\mathrm{~F}$ & Provence-Alpes-Côte d'Azur & $44 \cdot 42606$ & 6.88950 & 1951 \\
\hline 214 & $\mathrm{~F}$ & Provence-Alpes-Côte d'Azur & $44 \cdot 41444$ & 6.88917 & 2020 \\
\hline 230 & I & Piemonte & 44.15920 & $7 \cdot 57209$ & 1581 \\
\hline 231 & $\mathrm{~F}$ & Provence-Alpes-Côte d'Azur & 44.09426 & $7 \cdot 35626$ & 1873 \\
\hline 232 & $\mathrm{~F}$ & Provence-Alpes-Côte d'Azur & 44.09839 & $7 \cdot 31020$ & 2122 \\
\hline 233 & $\mathrm{~F}$ & Provence-Alpes-Côte d'Azur & 44.12946 & 6.96878 & 2185 \\
\hline 234 & $\mathrm{~F}$ & Provence-Alpes-Côte d'Azur & $44 \cdot 16116$ & 6.71292 & 1805 \\
\hline 235 & $\mathrm{~F}$ & Provence-Alpes-Côte d'Azur & 44.16689 & 6.70763 & 1930 \\
\hline
\end{tabular}




\section{Chapter 2}

\section{Pathways to polyploidy: indications of a female triploid bridge in the alpine species Ranunculus kuepferi (Ranunculaceae)}

Christoph C. F. Schinkel, Bernhard Kirchheimer, Stefan Dullinger, Danny Geelen, Nico De

Storme and Elvira Hörandl

Published in Plant Systematics and Evolution (2017) 303:1093-1108

DOI 10.1007/soo6o6-017-1435-6

Plant Syst Evol

DOI $10.1007 /$ s00606-017-1435-6

ORIGINAL ARTICLE

Pathways to polyploidy: indications of a female triploid bridge in the alpine species Ranunculus kuepferi (Ranunculaceae)

Christoph C. F. Schinkel ${ }^{1} \cdot$ Bernhard Kirchheimer $^{2} \cdot$ Stefan Dullinger $^{2}$.

Danny Geelen ${ }^{3} \cdot$ Nico De Storme $^{3} \cdot$ Elvira Hörandl ${ }^{1}$

Received: 29 September 2016/Accepted: 14 June 2017

(-) The Author(s) 2017. This article is an open access publication

Abstract Polyploidy is one of the most important evolutionary processes in plants. In natural populations, polyploids usually emerge from unreduced gametes which either fuse with reduced ones, resulting in triploid offspring (triploid bridge), or with other unreduced gametes, resulting in tetraploid embryos. The frequencies of these two pathways, and male versus female gamete contiblions, however, a largly occurs with diploid viploid und autetraplid cyolypes the Alps, wherey diploids are mosty sexul, whe terahids plons are facultative apomicts. To test for the occurrence of polyploidization events by triploid bridge, we investigated 551 plants of natural populations via flow cytometric seed screening. We assessed ploidy shifts in the embryo to reconstruct female versus male gamete contributions to polyploid embryo and/or endosperm formation. Seed formation via unreduced egg cells ( $B_{\text {III }}$ hybrids) occurred in all three cytotypes, while only in one case both gametes were unreduced. Polyploids further formed seeds with

Handling Editor: Ales Kovarik.

Electronic supplementary material The online version of this article (doi.10.1007/5006-1450 contains supplementary

Elvira Hörandl

elvira.hoerandl@ biologie.uni-goettingen.de

Department of Systematics, Biodiversity and Evolution of

Department of Botany and Biodiversity Research, University of Vienna, Rennweg 14, 1030 Vienna, Austria

Department of Plant Production, Faculty of Bioscience Engineering, Ghent University, Coupure Links 653 , 9000 Ghent, Belgium reduced, unfertilized egg cells (polyhaploids and aneuploids). Pollen was highly variable in diameter, but only pollen $>27 \mu \mathrm{m}$ was viable, whereby diploids produce higher proportions of well-developed pollen. Pollen size was not informative for the formation of unreduced pollen. These results suggest that a female triploid bridge via unreduct

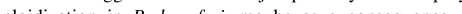
ploidizaton in $R$. kis the major pay may toward polyconstraints of endosperm development. Thiploids resulting from unreduced male gametes were not observed, whic explains the lack of obligate sexual tetraploid individual and populations. Unreduced egg cell formation in diploids represents the first step toward apomixis.

Keywords Apomixis - Flow cytometric seed screen Pollen size · Polyploidy · Ranunculus kuepferi · Triploid bridge

Introduction

Polyploidization is generally defined as the acquisition of more than the two nuclear chromosome sets found in diploids. Discovered more than a century ago (Strasburger 1910) and naturally occurring in many eukaryotic taxa (Wood et al. 2009) polyploidy is nowadays recognized as an evident and important force of evolution (Ramsey and Schemske 1998), especially in plants (Karpechenko 1927; Stebbins 1950, 1971) and particularly in angiosperm (Soltis and Soltis 2000; Wendel 2000). Fossil records indicate hat up to $70 \%$ of angiospen plants are of polyploid origin (Masterson 1994). More recent genomic analyses even suggest higher proportions of ancient polyploidization events in species 'histories to the point that all seed plants are estimated to be paleopolyploid (Blanc et al. 


\section{Abstract}

Polyploidy is one of the most important evolutionary processes in plants. In natural populations, polyploids usually emerge from unreduced gametes which either fuse with reduced ones, resulting in triploid offspring (triploid bridge), or with other unreduced gametes, resulting in tetraploid embryos. The frequencies of these two pathways, and male versus female gamete contributions, however, are largely unexplored. Ranunculus kuepferi occurs with diploid, triploid and autotetraploid cytotypes in the Alps, whereby diploids are mostly sexual, while tetraploids are facultative apomicts. To test for the occurrence of polyploidization events by triploid bridge, we investigated 551 plants of natural populations via flow cytometric seed screening. We assessed ploidy shifts in the embryo to reconstruct female versus male gamete contributions to polyploid embryo and/or endosperm formation. Seed formation via unreduced egg cells ( $B_{I I}$ hybrids) occurred in all three cytotypes, while only in one case both gametes were unreduced. Polyploids further formed seeds with reduced, unfertilized egg cells (polyhaploids and aneuploids). Pollen was highly variable in diameter, but only pollen $>27 \mu \mathrm{m}$ was viable, whereby diploids produced higher proportions of well-developed pollen. Pollen size was not informative for the formation of unreduced pollen. These results suggest that a female triploid bridge via unreduced egg cells is the major pathway toward polyploidization in R. kuepferi, maybe as a consequence of constraints of endosperm development. Triploids resulting from unreduced male gametes were not observed, which explains the lack of obligate sexual tetraploid individuals and populations. Unreduced egg cell formation in diploids represents the first step toward apomixis.

\section{Introduction}

Polyploidization is generally defined as the acquisition of more than the two nuclear chromosome sets found in diploids. Discovered more than a century ago (Strasburger 1910) and naturally occurring in many eukaryotic taxa (Wood et al. 2009) polyploidy is nowadays recognized as an evident and important force of evolution (Ramsey and Schemske 1998), especially in plants (Karpechenko 1927; Stebbins 1950, 1971) and particularly in angiosperms (Soltis and Soltis 2000; Wendel 2000). Fossil records indicate that up to $70 \%$ of angiosperm plants are of polyploid origin (Masterson 1994). More recent genomic analyses even suggest higher proportions of ancient polyploidization events in species' histories to the point that all seed 
plants are estimated to be paleopolyploid (Blanc et al. 2003; Jiao et al. 2011). This further indicates the importance for whole genome doubling (WGD) as driver for adaptation and speciation (Levin 1983; Soltis and Soltis 2009; Wood et al. 2009).

Besides many possible associated costs, the fundamental life history changes frequently accompanying the genomic excess of polyploidy, such as phenotypic and/or physiological plasticity, can convey polyploids an evolutionary advantage over their diploid progenitors ( $\mathrm{He}-$ garty and Hiscock 2008). Rapid range expansions of recently formed polyploids (Levin 1983; Hull-Sanders et al. 2009; Treier et al. 2009) and distinctly larger distribution areas of apomictic polyploids (van Dijk 2003; Kearney 2005; Hörandl 2006; Hörandl et al. 2008; Randle et al. 2009) which mostly extend to higher and accordingly cooler latitudes and elevations (Bell 1982; Bierzychudek 1985; Schinkel et al. 2016), are the most intriguing indications of such benefits.

However, despite the seemingly striking evolutionary importance of polyploidy, even the most crucial steps of polyploid evolution, their formation and establishment, are not fully understood. Although genetic events leading to polyploidy can occur frequently (Soltis and Soltis 1999; Soltis et al. 2004), neopolyploids have to overcome certain barriers to form viable populations and to persist (Levin 1975). Baack et al. (2005) pointed out that polyploidization must take place within diploid progenitor populations (sympatric), leaving them prone to pollen flow from diploids. The resulting triploids are assumed of lower fitness. According to the minor cytotype exclusion theory, neotetraploids would thus be quickly eliminated from the population (Levin 1975; Husband 2000). In this context, one major side effect of polyploidization is the immediate establishment of strong post-zygotic barriers, a mechanism leading to reproductive isolation (Ramsey and Schemske 1998, 2002). Alternatively, or in addition, polyploids may avoid genetic interaction with diploids by pre-zygotic barriers such as a switch to selfing or asexual modes of reproduction (Levin 1975), a phenological separation from their diploid ancestors through changes in flowering time (Segraves and Thompson 1999), or an ecological differentiation, i.e., by shifting the optima and/or expanding the breadths of their ecological niches (spatial separation) (Husband and Schemske 2000; Levin 2004). However, recent studies did not support niche divergence as an essential prerequisite for successful polyploid establishment (see Glennon et al. 2014, Kirchheimer et al. 2016).

Natural polyploid formation can occur through different pathways. Sexual polyploidization is usually based upon meiotic aberrations resulting in unreduced gametes, with pollen and/or 
egg cells exhibiting a somatic chromosome set (Bretagnolle and Thompson 1995; Ramsey and Schemske 1998; Brownfield and Köhler 2011; De Storme and Geelen 2013). Meiotic restitution is, similar to mitosis, a non-reductional cell division process in which dyads (and occasionally triads)-containing spores with the somatic chromosome number are produced instead of the tetrads-containing spores with the gametophytic chromosome number that are normally formed through regular meiotic cell division. Meiotic non-reduction has been recognized in numerous plant taxa and seems to be the predominant mechanism of diploid gamete formation (Bretagnolle and Thompson 1995; Bicknell and Koltunow 2004; Pecrix et al. 2011; De Storme et al. 2012; De Storme and Geelen 2013; Lovell et al. 2013; Mirzaghaderi and Hörandl 2016).

Meiotic restitution during female sporogenesis results in two unreduced megaspores, from which one develops into an unreduced embryo sac containing an unreduced egg cell and polar nuclei (diplospory). Female meiosis, however, can be also bypassed and an unreduced embryo sac can also develop from a somatic cell of the nucellus (apospory). These two mechanisms are components of gametophytic apomixis (Koltunow and Grossniklaus 2003). Nevertheless, asexual production of gametes is not exclusive and mostly accompanied by sexual gamete formation in parallel (Aliyu et al. 2010; Cosendai et al. 2013; Dobeš et al. 2013; Hojsgaard et al. 2013, 2014; Klatt et al. 2016). Simultaneous formation of unreduced and reduced gametes may result in sporadic merging of both types of gametes, depending on individual frequencies and spatial distribution. In particular, in apomictic species, the fusion of an unreduced egg cell with a reduced pollen grain constitutes a developmental pathway of partial apomixis utilizing a combination of unreduced embryo sac formation (apomeiosis) and subsequent fertilization. Such fertilized apomictic seeds result in the so-called $B_{\text {III }}$ hybrids (Nogler 1984; Bicknell et al. 2003), which cause polyploidization and are a potential pathway to fully functional apomixis when coupled to parthenogenesis (Hojsgaard et al. 2014). In contrast, polyploid induction resulting from the fertilization of a reduced egg cell by an unreduced pollen grain does not affect mode of reproduction and just results in sexual polyploidization (Mason and Pires 2015).

Incidence of these so-called $2 n$ gametes in natural populations is far more ubiquitous than previously thought. When involved in fertilization events, $2 n$ gametes directly produce polyploid offspring and are therefore considered a major pathway to polyploid formation (Bretagnolle and Thompson 1995; Ramsey and Schemske 1998; Otto and Whitton 2000; De 
Storme and Geelen 2013; Tayalé and Parisod 2013). Although production of unreduced gametes has been observed in many plant taxa, frequencies of unreduced egg cells are highly variable in seeds (Bicknell and Koltunow 2004; Sharbel et al. 2009; Aliyu et al. 2010; Hojsgaard et al. 2014; Klatt et al. 2016), as well as in sperm nuclei in pollen (Rani et al. 2013; Sora et al. 2016). Recent studies demonstrate that environmental stress, especially temperature, influences the production of $2 n$ gametes (Lokhande et al. 2003; De Storme et al. 2012; De Storme and Geelen 2013; Mirzaghaderi and Hörandl 2016). But also photoperiod (Quarin et al. 1986; Keller and Körner 2003; Kurepin et al. 2007; Klatt et al. 2016) and drought stress have been reported as factors driving $2 n$ gamete formation. These stressors can increase frequencies of unreduced embryo sac or pollen grain production to numbers sufficiently high to explain estimated rates of polyploid formation $\left(10^{-5}\right.$ for autotetraploids, $10^{-4}$ for allotetraploids; see Ramsey and Schemske 1998). However, bilateral sexual polyploidization events in which male and female unreduced gametes fuse have the joint probability of two rare events plus the imponderabilities of their temporal and spatial co-occurrence and thus are considered rare in natural populations (Ramsey and Schemske 1998; Husband 2004). Contrary to onestep polyploidization (tetraploid induction), the relatively high incidence of triploids in natural populations of many plant taxa (Dobeš et al. 2004; Husband 2004; Schranz et al. 2005i Voigt et al. 2007) suggests an alternative pathway of autotetraploid formation in two steps, with triploids serving as intermediates (Ramsey and Schemske 1998; Husband 2004). This process, called triploid bridge, involves the fusion of an unreduced with a regularly reduced gamete of diploid parent plants producing triploid offspring. In turn, these triploid intermediates may possibly generate tetraploid offspring through selfing or through crossing with either diploid progenitors or other triploids, depending on their fertility (Husband 2004; Köhler et al. 2010; Mason and Pires 2015).

Triploid bridges as pathway to neotetraploid formation may be hampered by triploid blocks (Comai 2005; Köhler et al. 2010). In some plant species, the parental chromosome dosage in the endosperm seems to be critical for seed development and fertility. Deviations from the usual 2:1 ratio between maternal and paternal chromosome contributions disorganize the expression of parentally imprinted genes necessary for normal endosperm development (Köhler et al. 2010; Lu et al. 2012; Haig 2013). Seeds with vestigial endosperm tend to abort or are mostly infertile (Brink and Cooper 1947). Hence, the sexual fusion of a haploid reduced 
with a diploid unreduced gamete results in a seed with a triploid embryo but imbalanced endosperm that probably blocks triploid seed formation (Ramsey and Schemske 1998; Spielmann et al. 2003). Moreover, in case triploid seeds do survive, the unequal chromosome number and severe difficulties regarding chromosomal pairing and segregation in meiosis make triploids gametophytically unstable, producing a variety of euploid $(1 n, 2 n$, or $3 n)$ and aneuploid gametes (Husband 2004). Lower frequencies of euploid gametes in turn may block tetraploid formation (Ramsey and Schemske 1998). Ramsey and Schemske (1998) evaluated that viable triploids in natural populations produced a surprisingly large amount of fertile euploid gametes. However, this statement refers primarily to pollen, for which a mean fertility of $31.9 \%$ is reported in triploid angiosperms. Reliable figures for female gametes are scarce (Bicknell and Koltunow 2004; Parisod and Besnard 2007; Sharbel et al. 2009; Schinkel et al. 2016; Wang et al. 2016) and need to be further investigated.

In this context, our study explores the possible occurrence of triploid bridges and triploid blocks among natural populations of the alpine plant Ranunculus kuepferi. The species occurs with diploid, triploid and autotetraploid cytotypes in the Alps, whereby diploids are mostly sexual, while tetraploids are facultative apomicts (Cosendai and Hörandl 2010; Schinkel et al. 2016). Previous studies of the species focused on the distribution of modes of reproduction, but the mode of polyploidization in this species was so far unknown. The observation that triploids occur in the contact zone of diploids and tetraploids prompted us to investigate whether these represent examples of a triploid bridge for polyploidization or just result from secondary backcrosses of established tetraploids with diploids. The amount of unreduced pollen formation in R. kuepferi was so far not investigated.

The aim of this study is (1) to analyze ploidy shifts in the seeds of diploid, triploid and tetraploid mother plants via flow cytometric seed screening; $(2)$ to analyze pollen size as putative indicator of unreduced male gamete formation; (3) to evaluate results with respect to the occurrence of a female versus a male triploid bridge-based polyploidization, putatively antagonized by endosperm imbalances acting as a triploid block. Finally, we will discuss implications of our results for the evolution of polyploidy and apomixis. 


\section{Materials and methods}

\section{a) Plant material}

Living plants of Ranunculus kuepferi were collected from 81 populations throughout the Alps and transferred to the Botanical Garden of the University of Goettingen as previously described by Schinkel et al. (2016). Our sampling included 18 diploid, 52 tetraploid and 11 populations with mixed cytotypes ( $2 x, 3 x, 4 x$ and $5 x$ ) (Schinkel et al. 2016). The mixed populations occur in the contact zone of diploids and tetraploids. For details on localities and collectors see Schinkel et al. (2016). Voucher specimens have been deposited in the herbarium GOET. In total, 1074 plants have been collected in early fruiting stage. Hence, ovule development, fertilization and seed formation had been completed under natural conditions in buds before collection (Schinkel et al. 2016), which is a prerequisite for analysis of developmental pathways without influences by artificial stress (e.g., cut out, transfer). Due to poor seed set in many individuals, we restricted sampling of seeds for later analysis to 551 plants with a minimum of at least five well-developed seeds. Mature achenes were gathered by bagging fruiting heads in perforated plastic pouches and were kept at room temperature for later analysis.

\section{b) Flow cytometry}

Determination of the somatic ploidy level of all mother plants was performed by standard methods using $0.5 \mathrm{~cm}^{2}$ fresh leaf material per individual (Schinkel et al. 2016). To discriminate between sexual and apomictic pathways in seed development, we used flow cytometric seed screening (Matzk et al. 2000) with minor modifications (Schinkel et al. 2016). Within most facultative apomicts, a single plant is capable of producing both sexual and apomictic seeds even inside the same flower (e.g., Aliyu et al. 2010; Dobeš et al. 2013). Based on this, we realized quantification of reproduction modes by determining the ploidy of both the endosperm and the embryo on a single seed level. Leaf samples of Zea mays strain CE-777 provided by J. Doležel were used as external standard and referenced every 96 runs.

For each plant, the ploidy level pattern of five seeds was analyzed following a two-step protocol by Doležel et al. (2007) using Otto I and Otto Il buffers, conducted on a CyFlow Space (Partec, Münster, Germany) with FloMax 2.2.0 (Quantum Analysis GmbH, Münster, Germany) operating software. Peak ranges were set manually and calculated as Gaussian means. 
Calculated ratios between endosperm and embryo ploidies provided a basis for discrimination between sexual (3:2) and apomictic (3:1, 2.5:1, 2:1) development without ploidy shifts in the embryo. For the respective peak ratios, a threshold of 1.65 was used to distinguish between sexual and apomictic seed. More detailed examinations of plausible developmental pathways followed Matzk et al. (2000), Talent and Dickinson (2007), Cosendai and Hörandl (2010) and Dobeš et al. (2013) and were adjusted accordingly (Schinkel et al. 2016).

Here, in this study, we specifically evaluated cases of ploidy shifts in the embryo. Results were pooled for ploidy levels of the mother and developmental pathways (Table 1). Seeds were categorized (after Nogler 1984; Bicknell et al. 2003) either as B BII hybrids (unreduced egg cell fertilized by reduced pollen = female triploid bridge; we apply this term to all cytotypes), polyhaploids (reduced egg cell of tetraploid without fertilization), disturbed sexuals (irregular male or female meiosis resulting in aneuploidy), biparental polyploidization (unreduced egg cells fertilized by unreduced pollen) or male triploidization (reduced egg cell fertilized by unreduced pollen). These cases can be discriminated by the respective embryo/endosperm ploidy pattern analysis (when ploidy of the mother plant was known), see flow histograms in Fig. 1 and interpretations in Table 1. Terminology for denotation of ploidy levels after DNA content and especially maternal as well as paternal genome contributions followed Greilhuber et al. (2005). 
Table 1 Seeds with ploidy shifts in the embryo according to flow cytometric seeds screening (for seeds without ploidy shifts see Schinkel et al. 2016)

\begin{tabular}{|c|c|c|c|c|c|c|c|}
\hline \multicolumn{5}{|l|}{ Ploidy } & \multirow[t]{2}{*}{ Endosperm ratio (maternal/paternal) } & \multirow[t]{2}{*}{ Reproduction mode } & \multirow[t]{2}{*}{$\mathrm{N}$} \\
\hline Mother plant & Egg cell & Sperm nuclei & Embryo & Endosperm & & & \\
\hline \multicolumn{8}{|l|}{ Diploid } \\
\hline $2 x$ & $2 x$ & $1 x$ & $3 x$ & $5 x$ & $4: 1$ & BIII & 3 \\
\hline \multirow[t]{2}{*}{$2 x$} & $2 x$ & $2 x$ & $4 x$ & $6 x$ & $2: 1$ & Biparental polyploidization & 1 \\
\hline & & & & & & & (Total 4) \\
\hline \multicolumn{8}{|l|}{ Triploid } \\
\hline $3 x$ & $1 x$ & $1 x$ & $2 x$ & $3 x$ & $2: 1$ & Irregular female meiosis & 2 \\
\hline $3 x$ & $3 x$ & $1 x$ & $4 x$ & $7 x$ & $6: 1$ & BIII & 2 \\
\hline $3 x$ & $3 x$ & $1 x$ & $4 x$ & $9 \times a$ & 1:ob & BIII & 1 \\
\hline \multirow[t]{2}{*}{$3 x$} & $3 x$ & $1 x$ & $4 x$ & $10 \times a$ & $9: 1$ & BIII & 1 \\
\hline & & & & & & & (Total 6) \\
\hline \multicolumn{8}{|l|}{ Tetraploid } \\
\hline $4 \times$ & $2 x$ & ox & $2 x$ & $6 x$ & $2: 1$ & Polyhaploid & 12 \\
\hline $4 x$ & $2 x$ & $1 x$ & $3 x$ & $5 x$ & $4: 1$ & Irregular male meiosis & 2 \\
\hline $4 x$ & $2 x$ & $1 x$ & $3 x$ & $6 \times a / d$ & 1:ob & $"$ & 1 \\
\hline $4 x$ & $2 x$ & $1 x$ & $3 x$ & $7 \times a$ & $3: 1$ & $"$ & 4 \\
\hline $4 x$ & $3 \times c$ & $2 x$ & $3 x$ & $8 x$ & $3: 1$ & Irregular female meiosis & 3 \\
\hline $4 x$ & $4 x$ & $2 x$ & $6 x$ & $10 x$ & $4: 1$ & BIII & 10 \\
\hline \multirow[t]{2}{*}{$4 x$} & $4 x$ & $2 x$ & $6 x$ & $14 \times a$ & $6: 1$ & BIII & 1 \\
\hline & & & & & & & (Total 33) \\
\hline
\end{tabular}

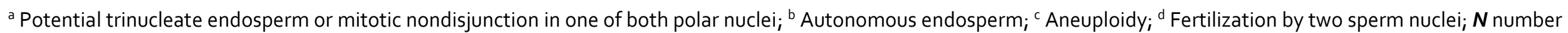
of seeds 

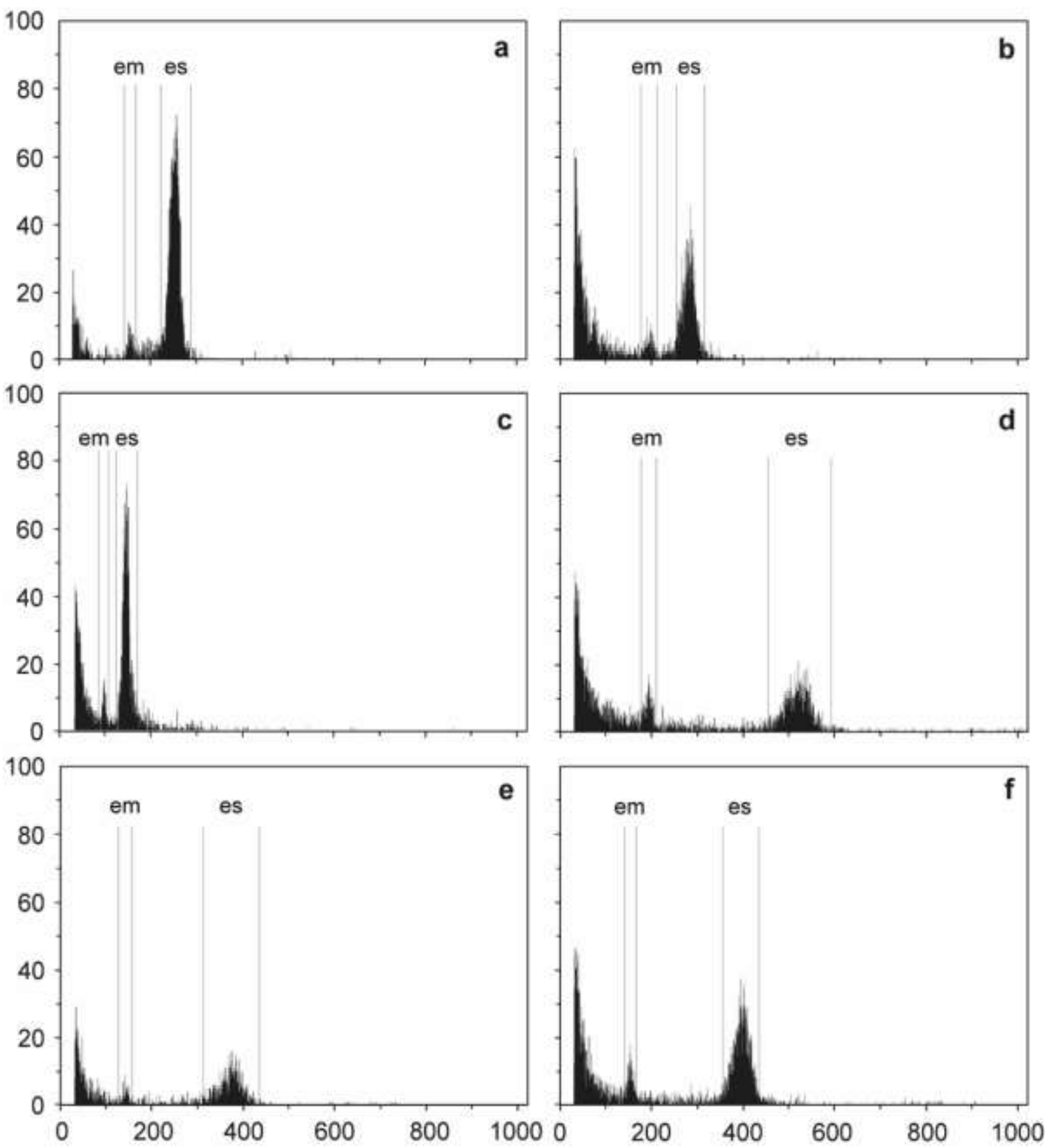

Fig. 1. Flow histograms of six seed formation pathways with ploidy shifts in the embryo inferred from FCSS in Ranunculus kuepferi (see Table 1). a Uniparental polyploidization on a diploid mother plant, triploid embryo, pentaploid endosperm (first step in tetraploidization via female triploid bridge); $\mathbf{b}$ biparental polyploidization with diploid mother plant, tetraploid embryo, hexaploid endosperm (one-step tetraploidization or $2 n p o l l e n$ from tetraploid pollen donor); c progeny from triploid mother plant with diploid embryo, triploid endosperm; $\mathbf{d}$ uniparental polyploidization with triploid mother plant, tetraploid embryo and decaploid endosperm, indicating trinucleate es (second step in tetraploidization via female triploid bridge); e polyhaploid progeny from tetraploid mother plant with triploid embryo, hexaploid endosperm (trinucleate es or es fertilization with 2 sperm nuclei); f triploid embryo with octaploid endosperm from a tetraploid mother plant, indicating irregular female meiosis; em embryo peak; esendosperm peak 


\section{c) Triploid bridge and triploid block}

Due to the availability of maternal leaf ploidy data for all plants, embryos with higher ploidy than the mother plant could be determined with high certainty. To investigate hypothetical presence of a triploid bridge in R. kuepferi, we assessed the occurrence of triploid embryos among diploid mother plants, as well as tetraploid embryos derived from triploid plants as potential evidence. Focusing on maternal-to-paternal genome ratios in endosperm, deviations from the normal 2:1 state were interpreted as potential triploid block. According to Harlan and de Wet (1975), tetraploidy principally can be reversible which is part of polyploid evolution. Tetraploids producing lower- or even higher-ploidy offspring may be detrimental for survival due to minor cytotype exclusion as a function of ploidy shift frequency. Hence, we also documented all cases of tetraploids that had produced embryos of divergent ploidies. Triploid progeny of tetraploids, if not based upon fertilized reduced egg cells, suffers from aneuploidy and chromosomal deficiencies that may enhance implications of a potential triploid block.

\section{d) Pollen size and stainability}

Three-dimensional pollen size (or volume) was determined on a Multisizer 3 (Beckman Coulter, Brea, California, United States) following pollen preparation methods described by De Storme et al. (2012) with minor modifications. From 179 R. kuepferi plants, mature stamens from the outer rim of single flowers were collected, dried in silica gel and cut in half prior to soaking them for at least 15 min in 5 ml ISOTON II (Beckman Coulter, Brea, California, United States) in accuvette cups (Beckman Coulter, Brea, California, United States). To check for any significant influence of incubation time on pollen size, we conducted time series analyses of randomly chosen pollen samples (3 di- and 4 tetraploid) measuring same samples 9 times, with 15 min time between the distinct measurements (120 min). These test series revealed no significant change of mean pollen diameter $(P=0.59)$ and pollen size distribution $(P=0.43)$ among samples (Online Resource 1), indicating that incubation time does not influence the pollen size. At least 10,000 particles per sample were counted of which approximately 2,0003,000 were within the estimated pollen size range. Histogram peaks of pollen diameters were generated by and analyzed with the Multisizer 3 Control Software 3.53 (Beckman Coulter, Brea, California, United States). 
For microscopy, mature pollen grains were stained with a 10\% acetic orcein solution for 15 min and viewed with a Zeiss Apotome 2 microscope (Carl Zeiss AG, Oberkochen, Germany) at $400 \times$ magnification. At least ten stamens per plant were analyzed, and diameters of 80120 pollen grains per sample (depending on quality and quantity of available anthers) were measured with ZEN operating software (Carl Zeiss AG, Oberkochen, Germany). To determine the different size classes of viable pollen, we further carried out a stainability test, using a $10 \% \mathrm{I}_{2}-\mathrm{KI}$ solution (Lugol's iodine) for detection of starch content as an indicator for mature viable pollen (Wang et al. 2004). A light microscope (Leica DM5500B with DFC 450 C camera, LAS V 41 Software, Leica Microsystems, Wetzlar, Germany) at a $400 x$ magnification was used to discriminate black-stained, viable pollen from brownish, reddish and translucent (empty) pollen which were all considered as non-viable (Stebbins 1950; Tie et al. 2006).

\section{e) Statistical analyses}

Independent $T$ and $F$ tests were performed to check for significant differences in pollen size and respective proportions among size classes between diploids and tetraploids. All calculations were executed in R version 3.1.2 (R Core Team 2014). Prior to analysis, percentages were arcsine-transformed to improve normal distribution of the data.

\section{Results}

\section{a) Flow cytometric seed screening}

The ploidy level of 551 individual mother plants (132 diploid, 25 triploid and 394 tetraploid) and the embryo/endosperm ploidy pattern in 2795 seeds were determined. The great majority of seeds had no ploidy shift in the embryo compared to the mother plant and was either formed from a sexual or a fully apomictic pathway, resulting in diploid and tetraploid embryos, respectively (see details and representative flow histograms in Schinkel et al. 2016). We present here 43 seeds ( $1.5 \%$ of all seeds) showing a shift in the ploidy level of the embryo compared to the mother plant (see Online Resource 2). Four of these seeds occurred in diploid, six in triploid and 33 in tetraploid mother plants (Table 1 and Fig. 2). Three seeds of diploid mother plants contained a triploid ( $3 x$ ) embryo accompanied by a pentaploid ( $5 x$ ) endosperm (B $B_{\| 1}$ hybrids). The observed ratio indicates for the involvement of unreduced female gametes, resulting in a diploid egg cell $(2 C x(m))$ fertilized by one haploid sperm nucleus of a 
reduced pollen $\left({ }_{1} C x(p)\right)$, as well as two fused diploid polar nuclei $\left(2 C x(m)+{ }_{2} C x(m)={ }_{4} C x(m)\right)$ fertilized by the second haploid sperm nucleus $(1 C X(p))$.

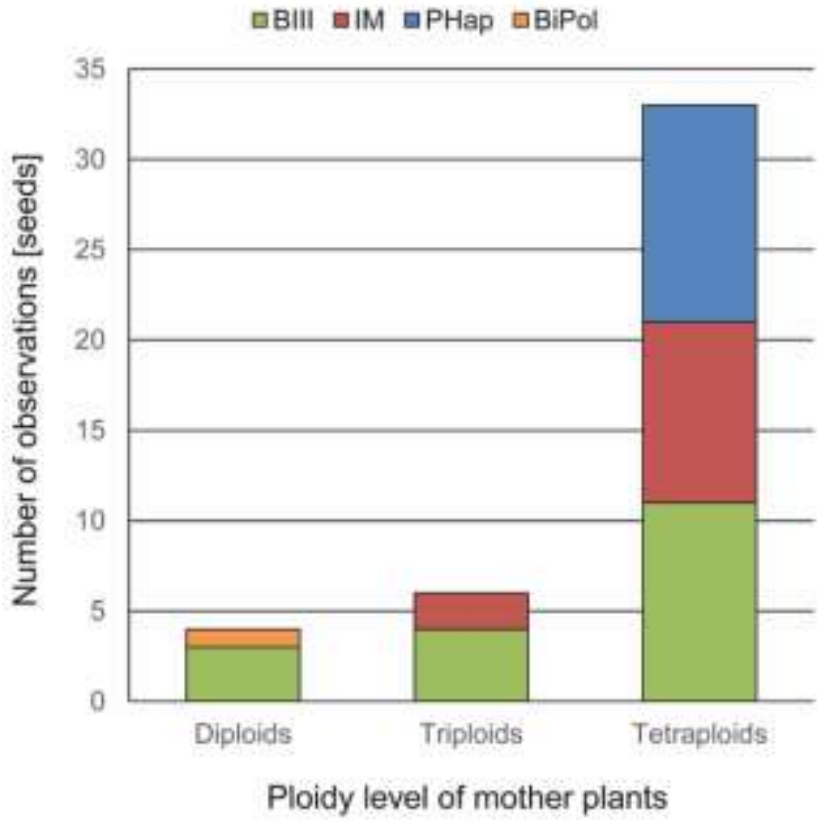

Fig. 2 Barplot of seed formation pathways among the 43 seeds with ploidy shifts in the embryo inferred from FCSS in Ranunculus kuepferi (see Table 1). Pathways were grouped in four different classes based on main reproduction mode. BIII BIII hybrids; IM irregular meiosis; PHap polyhaploids; BiPol biparental polyploidization

The fourth seed contained a tetraploid $(4 x)$ embryo together with a hexaploid $(6 x)$ endosperm, indicating the additional involvement of an unreduced pollen grain. Here, the endosperm had a ratio of $4 C x(m): 2 C x(p)$, resembling the optimal maternal-paternal allocation of 2:1 (Lin 1984). Strikingly, no triploid resulting from the fusion of an unreduced pollen with a reduced embryo sac could be found, as this would show an embryo/endosperm ratio of 3:4 $(1 C x(m)+2 C x(p)$ in the embryo, and $2 C x(m)+2 C x(p)$ in the endosperm. Of the six cases of ploidy shifts of the embryo in triploid mother plants (found within 125 analyzed seeds), four seeds contained tetraploid $(4 x)$ embryos. Another two seeds contained diploid $(2 x)$ embryos, both with triploid $(3 x)$ endosperm indicating double fertilization by reduced pollen of either a diploid or a likewise triploid donor (euploid $1 x$ pollen).

For the 33 seeds with embryo ploidy shifts from their tetraploid mothers, the resulting combinations of embryo and endosperm ploidy and the associated developmental pathways were rather complex (see Table 1). We found 12 seeds exhibiting diploid embryos with hexaploid endosperm, likely a consequence of polyhaploid embryo formation (reduced, unfertilized egg cell, the polar nuclei fertilized with two reduced sperm nuclei). Ten triploid embryos with endosperm ploidies ranging from penta- to octaploid can be explained by irregular female or male meiosis as observed previously by Cosendai and Hörandl (2010). Eleven seeds had hexaploid embryos with either deca- or tetradecaploid endosperm, representing $B_{I I I}$ hybrids with unreduced female gamete formation. Explanations of some higher-ploidy endosperm in tetra- $(6 x, 7 x$ for diploid embryos, $14 x$ for hexaploid embryos) as well as triploids ( $9 x$, 
10x) remain elusive, although according to Talent and Dickinson (2007) trinucleate endosperm could explain some of the observed patterns.

\section{b) Pollen analysis}

Pollen size measurements revealed a large spectrum of diameters in both tetraploid and diploid progenitor plants. Ranges in both were very similar, varying from 15.8-39.7 $\mu \mathrm{m}$ in tetraploids and $15.6-38.3 \mu \mathrm{m}$ in diploids (Fig. 3). Histograms of most samples peaked at approximately the same four sizes (Fig. 4). Therefore, we assorted observed counts obtained from the histograms into four size classes (Table 2 ) of very small $(A)$, small $(B)$, larger $\left(C_{1}\right)$ and very large (C2) pollen. The classification is based on our microscopic observations, which revealed pollen grains of varying sizes and abundance reasonably consistent with the volumetric measurements (Fig. 5). Particles smaller than $19 \mu \mathrm{m}$ were either strongly deformed, were deeply grooved pollen or represented immature pollen in development as well as cell debris from preparation. Particles in the range from 19 to $27 \mu \mathrm{m}$ appeared to be empty pollen seemingly stuck in development. Only particles greater than $27 \mu \mathrm{m}$ appeared to be fully developed pollen, showing common characteristics of viable tricolpate-psilate pollen as typical for many Ranunculusspecies (Huber 1988; Izmailow 1996; Hörandl et al. 1997). Everything above $33 \mu \mathrm{m}$ diameter looked like bloated pollen, sometimes exhibiting signs of disintegration, or was debris (e.g., larger tissue segments) in the solution. We did not observe pollen with incomplete tetrade disintegration or any form of other random pollen aggregation.

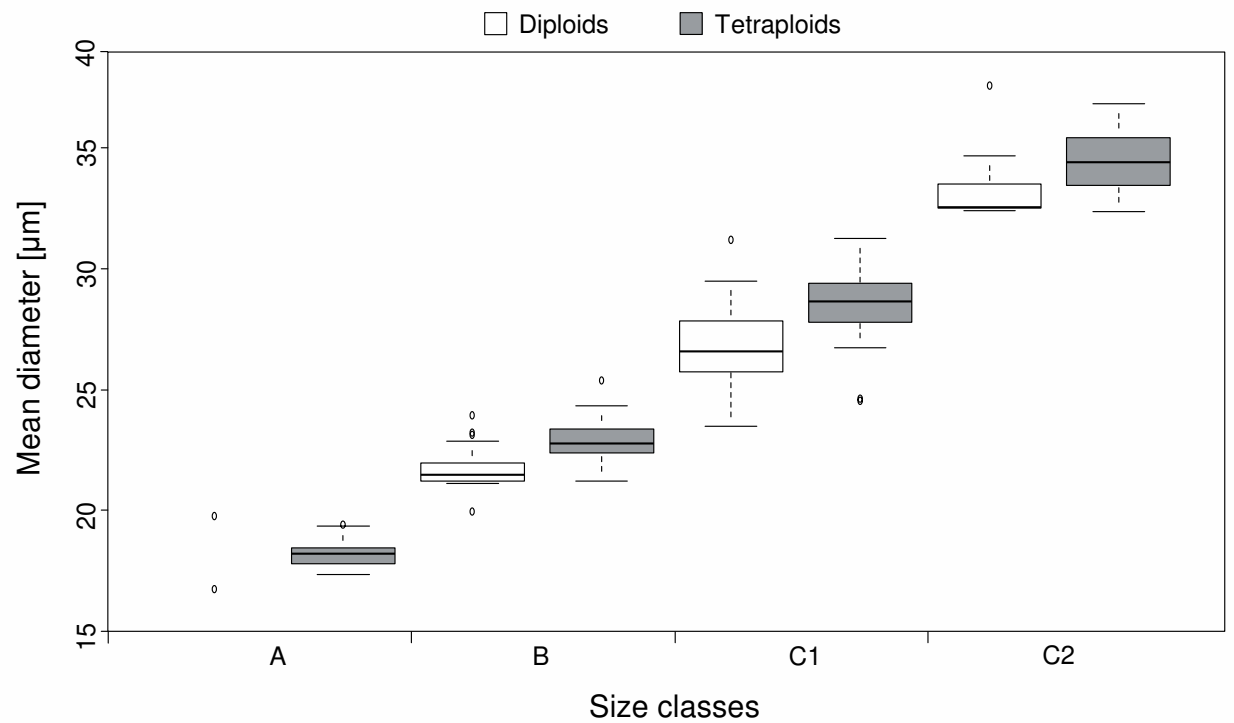


Fig. 3 Boxplot of volumetric pollen measurements in Ranunculus kuepferi (see Table 2). Results were calculated as diameters and grouped in four size classes. A: smallest, with diameters under $19 \mu \mathrm{m} ; \mathrm{B}$ : small, $19-27 \mu \mathrm{m}_{;} \mathrm{C}_{1}$ : large, 27-33 $\mu \mathrm{m} ;$ C2: largest, with diameters above $33 \mu \mathrm{m}$

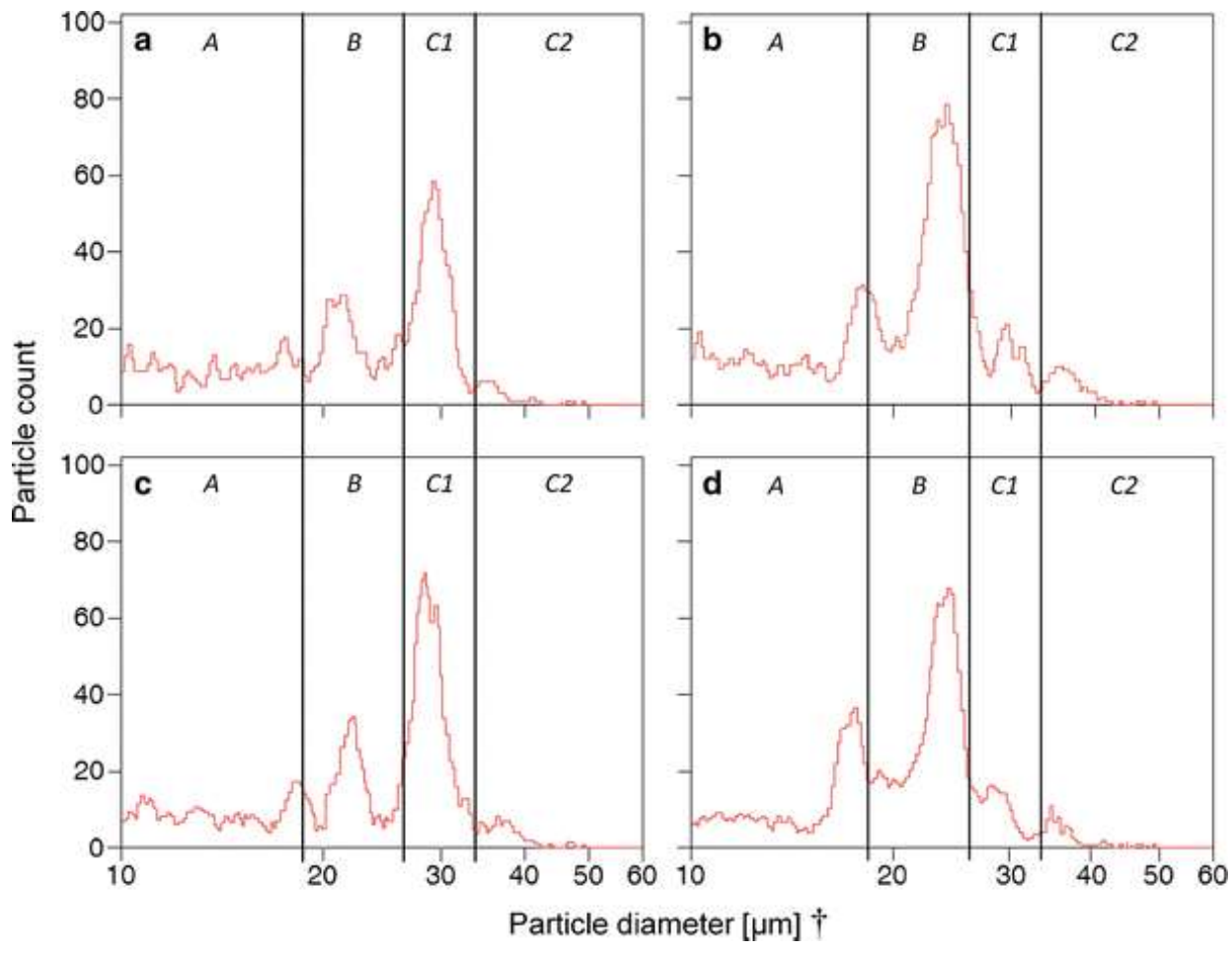

Fig. 4 Histograms of size classes of volumetric pollen measurements of two diploid $(\mathbf{a}, \mathbf{c})$ and two tetraploid (b, d) individuals. Vertical black lines show margins of size class ranges. $P K$ peak; ${ }^{\dagger}$ particle diameter was automatically calculated in Multisizer 3 Control Software 3.53 (Beckman Coulter, Brea, California, United States) based on volumetric measures 
Table 2 Deduced size classes, average sizes and proportions from pollen volumetric measurements

\begin{tabular}{|c|c|c|c|c|c|c|c|c|c|c|}
\hline & \multicolumn{4}{|c|}{ Beckman Coulter Multisizer 3} & \multicolumn{6}{|c|}{ Microscope } \\
\hline & $x \leq 19$ & $19<x \leq 27$ & $27<x \leq 33$ & $x>33$ & Aborted & Non-viable & $\begin{array}{l}\text { Viable, } \\
\text { small }\end{array}$ & $\begin{array}{l}\text { Viable, } \\
\text { large }\end{array}$ & Viable & $\begin{array}{l}\text { Non-via- } \\
\text { ble }\end{array}$ \\
\hline & $A$ & $B$ & $\mathrm{C}_{1}$ & $\mathrm{C}_{2}$ & $A$ & $B$ & $\mathrm{C}_{1}$ & $\mathrm{C}_{2}$ & & \\
\hline \multicolumn{11}{|l|}{ Diploids } \\
\hline$N$ & 15,184 & 53,841 & 112,859 & 9101 & 126 & 1925 & 3040 & 1416 & 2868 & 3639 \\
\hline$\varnothing N /$ individual & 287 & 1146 & 1710 & 1011 & 2 & 29 & 45 & 21 & 43 & 54 \\
\hline $\begin{array}{l}\text { Mean diameter } \\
{[\mu \mathrm{m}]}\end{array}$ & $18.3 \pm 0.6$ & $20.6 \pm 4.7$ & $26.7 \pm 1.5$ & $33.4 \pm 1.7$ & & & & & & \\
\hline Proportion [\%] & 8.0 & 28.2 & 59.1 & 4.8 & 1.9 & 29.6 & 46.7 & 21.8 & 44.1 & 55.9 \\
\hline \multicolumn{11}{|l|}{ Tetraploids } \\
\hline$N$ & 24,893 & 120,724 & 69,693 & 12,266 & 1463 & 4439 & 2396 & 1112 & 2892 & 6518 \\
\hline$\varnothing N /$ individual & 372 & 1548 & 882 & 371 & 16 & 49 & 27 & 12 & 32 & 72 \\
\hline $\begin{array}{l}\text { Mean diameter } \\
{[\mu \mathrm{m}]}\end{array}$ & $18.2 \pm 0.4$ & $22.6 \pm 2.4$ & $28.6 \pm 1.2$ & $34.4 \pm 1.3$ & & & & & & \\
\hline Proportion [\%] & 10.9 & 53.0 & 30.6 & 5.4 & 15.5 & 47.2 & $25 \cdot 5$ & 11.8 & 30.7 & 69.3 \\
\hline
\end{tabular}

Sizes correspond to diameters; size means and proportions are calculated with pooled results of all measured pollen per cytotype

$N$ total number of measurements (particles), $\varnothing N /$ individual mean number of measurements (particles) per individual 


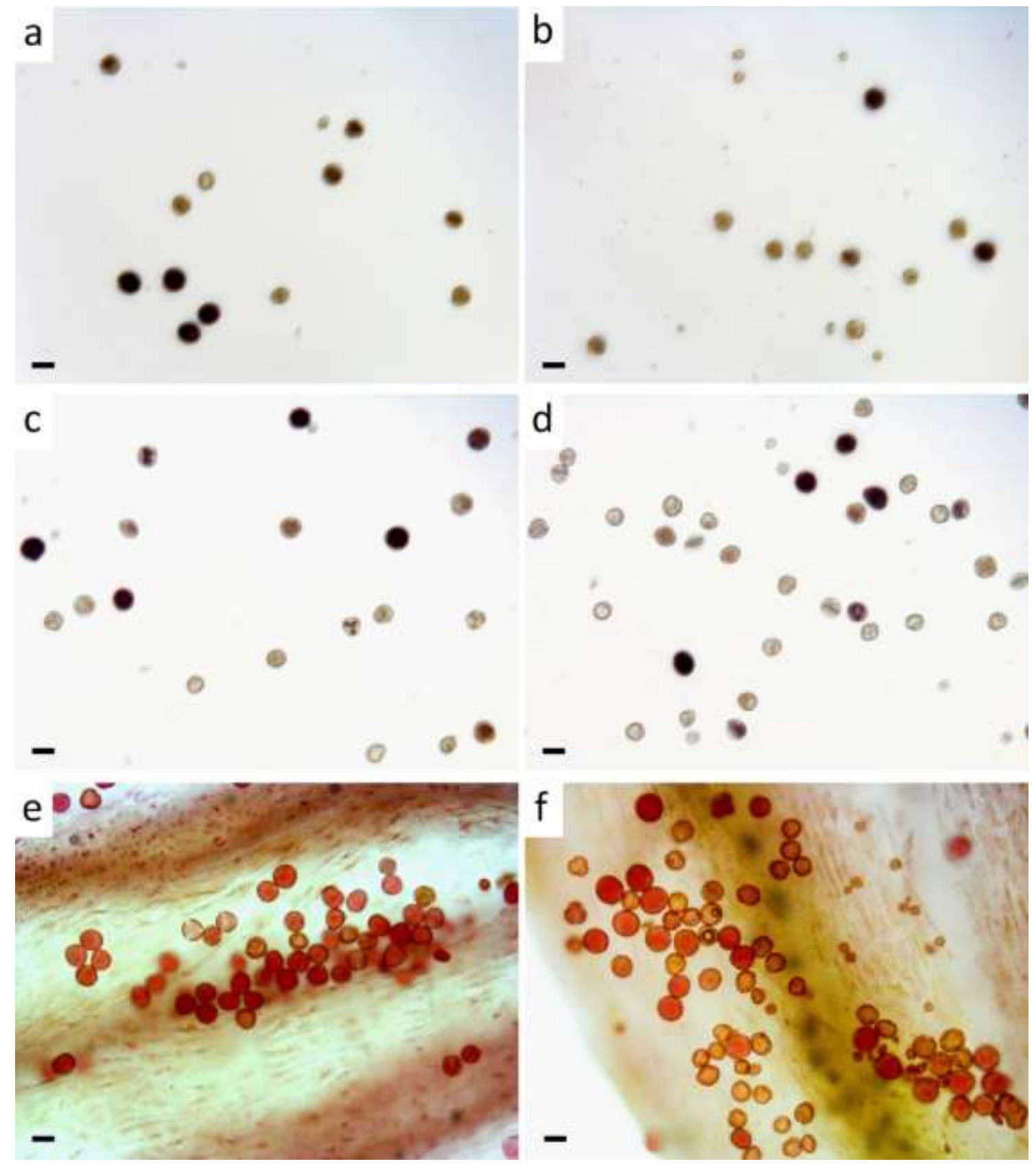

Fig. 5 Microscopic pictures showing pollen of Ranunculus kuepferi representing different size classes. Pollen was stained with either $10 \% \mathrm{I}_{2}-\mathrm{KI}$ solution (Lugol's iodine) (viability staining; a-d) or $10 \%$ acetic orcein (manual size measurement; e-f). Pollen from three diploid $(\mathbf{a}, \mathbf{c}, \mathbf{e})$ and three tetraploid $(\mathbf{b}, \mathbf{d}, \mathbf{f})$ plants are shown. For viability assessment with $\mathrm{I}_{2}-\mathrm{KI}$, pollen grains stained black were judged as viable, and those staining brownish to light yellowish were considered sterile (a-d). Acetic orcein pictures were taken directly from anthers and serve as visual illustration. Bar corresponds to $27 \mu \mathrm{m}$ (approximate delimitation between mostly non-viable [<27 $\mu \mathrm{m}$ ] and mostly viable $[>27 \mu \mathrm{m}]$ pollen)

Generally, average diameters of pollen isolated from diploids were smaller than those of tetraploids except for diameters in size class $A$, wherein counted particles were slightly but not 
significantly bigger in diploids $[t(89)=0.1, P=0.95]$. Observed differences were highly significant in size classes $B[t(89)=-7.2, P<0.01]$ and $C_{1}[t(89)=-3.3, P<0.01]$ (Table 2), whereas differences between the pollen from di- and tetraploids in class $C_{2}$ were only marginally significant $[t(89)=-2.2, P=0.04]$.

No significant difference in diploid/tetraploid proportions was found in class $A[F(151)=2.0$, $P=0.16]$ (Fig. 3). Classes $B$ and $C_{1}$ differed significantly with tetraploids comprising more of the smaller $\left(B_{i}[F(151)=16.7, P<0.01]\right)$ and diploids more of the larger $\left(C_{1} ;[F(151)=29.3, P<\right.$ 0.01]) pollen. Very large pollen of size class $C_{2}$ were significantly more frequent in tetraploids $[F(151)=5.5, P=0.02]$ compared to diploids.

Stainability analysis with Lugol's iodine revealed tetraploids to have significantly less viable pollen (mean of $30.7 \%$ ) compared to diploids (mean 44.1\%) $[t(66)=-4.76, P<0.01]$. Interestingly, in both cytotypes pollen with diameters smaller than $27 \mu \mathrm{m}$ was not well developed. Nearly all viable pollen observed during microscopy possessed diameters greater than $27 \mu \mathrm{m}$ and was therefore assigned to size classes $C_{1}$ and $C_{2}$.

\section{Discussion}

We here report neopolyploid formation via unreduced eggs in wild populations of the alpine plant Ranunculus kuepferi and call this pathway of polyploidization a female triploid bridge. Additional to the overall ploidy distribution and reproduction pathways that maintain the ploidy level (Schinkel et al. 2016), we revealed the events that lead to polyploid formation based on ploidy shifts in the seeds (embryos and endosperm) of di-, tri- and tetraploid mother plants.

\section{a) Developmental pathways}

Among diploid progenitors, we identified three of four seeds with triploid ( $3 x$ ) embryos and pentaploid $(5 x)$ endosperm derived from two fused diploid $(2 x)$ nuclei of the central cell fertilized by a reduced pollen sperm nucleus $(1 X)$. Hence, the unreduced egg cell and central cell have been fertilized each by the two haploid ( $1 x)$ sperm derived from a reduced pollen. Ramsey and Schemske (1998) found a similar pattern in a comparison of $4 x \times 2 x$ versus $2 x \times 4 x$ crosses.

Although these findings indicate that triploid bridge-mediated polyploidy induction in diploid $R$. kuepferi occurs through the formation of unreduced female gametes, most literature on 
restitutional meiosis assesses rates of unreduced $2 n$ pollen and neglects the process of female gametogenesis (Mirzaghaderi and Hörandl 2016). Flow cytometric seed screening informs about male vs. female unreduced gamete formation because embryo/endosperm ploidies are different. When a normal meiotically reduced haploid egg cell of a diploid mother plant is fertilized by an unreduced $2 n$ pollen, the resulting embryo would be triploid ( $3 x$ ) accompanied by an tetraploid ( $4 x$ ) endosperm possessing a 2:2 maternal-to-paternal genome balance ( $2 C x(m)$ from the two polar nuclei, $2 C x(p)$ from the second sperm nucleus). A key finding of our studies is that we did not find any case of such triploid seed, which indicates that no male triploidization by unreduced $2 n$ pollen took place in our sample. Moreover, these results also reveal that it is highly unlikely that triploids in the sympatric geographical zone originated from backcrossing of tetraploid pollen donors to diploid mother plants (see map in Schinkel et al. 2016). Tetraploids typically produce reduced pollen with a diploid chromosome set $(2 x)$. Events whereby diploid pollen fertilize the egg of a sexual diploid would also result in triploid embryos accompanied by tetraploid endosperm (3:4). However, we did not find a single seed with this ploidy structure. Hence, we cannot confirm the theoretical possibility of a secondary origin of triploids, which is further impeded by low fertility (Hörandl and Temsch 2009).

However, we also found evidence for $2 n$ pollen being involved in biparental polyploidization in $R$. kuepferi. One seed among diploids revealed a tetraploid ( $4 x)$ embryo and a hexaploid $(6 x)$ endosperm, which can only result from an unreduced diploid $(2 x)$ pollen that has fertilized both the egg $(2 x)$ and central cell $(4 x)$ of an unreduced $(2 x)$ embryo sac. Due to the involvement of both unreduced male and female gametes, the mechanism of autotetraploidization here would be a biparental process rather than a uniparental one, as would be the case if the polyploidization was entirely based on either unreduced female or male gametes. However, such biparental polyploidization may be also caused by reduced pollen of surrounding higher-ploidy individuals, including aneuploid pollen of triploids. This case is more likely as the seed was formed in a population of mixed ploidies in the contact zone of cytotypes (Schinkel et al. 2016).

It is generally thought that triploids have low fertility or even are totally sterile. Indeed, in many species, difficulties in the production of viable seeds and pollen restrict their role in tetraploid formation (Ramsey and Schemske 1998). However, recent studies that estimated fer- 
tility of triploids suggested that triploids in many taxa are able to produce some euploid gametes and are therefore often semi-fertile (Ramsey and Schemske 1998). Accordingly, in our previous study on $R$. kuepferi (Schinkel et al. 2016) we had obtained 125 fully developed seeds from 25 triploid plants, which we analyzed with DNA flow cytometry. Most seeds consisted of a triploid embryo and a pentaploid endosperm, when produced sexually (six seeds), or hepta- as well as octaploid endosperm, when produced apomictically (119 seeds). Although seed set in triploids is lowest among the three analyzed cytotypes (Schinkel et al. 2016), these findings suggests that triploids are indeed fertile to some degree, not least because they mainly reproduce via apomixis. Switching the reproductive path to apomixis is a common strategy of polyploid plants to escape sexual-based F1sterility (e.g., Hojsgaard et al. 2014) and thus constitutes an important factor in the establishment of a persistent amount of triploids among diploid populations, which is sufficient to allow further polyploidization steps. Focusing on the six seeds of triploid individuals that featured a ploidy shift of the embryo, four seeds possessed tetraploid ( $4 x$ ) embryos. Heptaploid $(7 x)$ endosperm of two of these seeds indicates an origin from unreduced egg cells fertilized by euploid reduced $1 x$ pollen of either a triploid or diploid donor. Such a pathway can be described as unilateral female sexual tetraploidization involving unreduced $(3 x)$ embryo sacs. The remaining two seeds with tetraploid embryos were accompanied by a nonaploid or a decaploid endosperm, respectively. In both cases, the most plausible explanation for the observed patterns is the presence of trinucleate endosperm (Talent and Dickinson 2007), since unreduced triploid pollen can explain nonaploid endosperm, but would cause hexaploid embryos. Certainly, trinucleate nonaploid endosperm implies that the endosperm developed autonomously, i.e., without fertilization (pseudogamy), as we previously found in some seeds of tetraploid individuals (Schinkel et al. 2016). In the case of decaploid endosperm, we assume a normal double fertilization of the unreduced egg cell and the trinucleate endosperm by a euploid reduced 1xpollen (diploid/triploid donor). Besides the four seeds comprising tetraploid embryos, two seeds were detected with diploid embryos and a triploid endosperm. Although comprising the same embryo/endosperm condition as regularly formed seeds of sexual diploids, it remains elusive whether these seeds would be fertile or not.

In comparison with triploids, an even larger variety in possible mating outcomes was observed in the progeny of tetraploid mother plants. Besides the most common, sexual and asexual reproduction without changes of embryo ploidy (Schinkel et al. 2016), we found 33 
seeds in which a ploidy shift in the embryo could be detected. Interestingly, most of these seeds (12) contained a polyhaploid embryo, i.e., resulting from a reduced, unfertilized egg cell (Table 1). The other seeds contained either again $B_{\text {III }}$ offspring (unreduced female gametes fertilized by reduced male gametes) or reduced, but triploid embryos and $6 x$ to $8 x$ endosperm (Table 1). This last developmental pathway can only be interpreted with an irregular female meiosis resulting in aneuploid gametes. Such seeds were also found regularly in a previous study within tetraploid populations outside the contact zone of diploids and tetraploids (Cosendai and Hörandl 2010). Their formation may be explained by multivalent formation and unequal chromosome segregation during meiosis in these autotetraploid plants, resulting in aneuploid megaspores (see Cosendai et al. 2011).

\section{b) Endosperm imbalance and triploid block}

As in other plants, triploids of $R$. kuepferi have a lower seed set than diploids and tetraploids (Schinkel et al. 2016). Seed quality is in most angiosperms strongly dependent on proper endosperm formation as it is the nutritious tissue for the embryo. The ratio of two maternal-topaternal genome copies appears to be optimal (Scott 2007; Köhler et al. 2010). Paternal genes proliferate endosperm growth, while maternal genes downregulate growth but are important for normal cellularization of endosperm (Spielmann et al. 2003). Genomic imprinting, i.e., the differential expression of paternally and maternally inherited genes, is another potential factor of endosperm imbalance (Haig and Westoby 1991; Vinkenoog et al. 2003; Köhler et al. 2010). Interploidy crosses affect the endosperm balance by altering maternal-to-paternal genome contributions. However, some pseudogamous apomicts developed mechanisms to overcome detrimental effects resulting from such deviations, for example conformation in megagametophyte structure and/or fertilization behavior, in particular dispermy (sexual polyploidization), as well as endosperm fertilization under inclusion of both sperm nuclei (Talent and Dickinson 2007; Šarhanová et al. 2012; Ludwig et al. 2013; Burgess et al. 2014). While the female triploid bridge via unreduced egg cells and polar nuclei will result in triploid embryos accompanied by an unbalanced endosperm containing a maternal-to-paternal genome ratio of $4 m: 1 p$, the male triploid bridge would result in a maternal-to-paternal genome ratio of $2 m: 2 p$ in the endosperm. In R. kuepferi, we only observed the pathway of unreduced female gamete formation, which may have less severe effects on endosperm (smaller, but cellularized) than the paternal excess (over-proliferated, but not cellularized; 
see Spielmann et al. 2003 on Arabidopsis). Observations of rare cases of autonomous endosperm formation in Cosendai and Hörandl (2010) and Schinkel et al. (2016) support the hypothesis that the species $R$. kuepferitolerates a lower paternal genome contribution to endosperm better than a paternal excess. Hence, these findings together with data presented in this study demonstrate the triploid block in R. kuepferi is not strict and still allows for triploid seed formation at low frequencies.

\section{c) Pollen ploidy}

In many plant taxa, pollen ploidy can directly be assessed by pollen size measurements (Ramsey and Schemske 1998). Hence, differences in diameter of reduced and unreduced pollen should be likewise detectable. Several studies reported that the diameter of $2 n$ pollen grains is often $30-40 \%$ larger than that of reduced pollen and that size distribution of reduced and unreduced pollen is mostly bimodal (e.g., Róis et al. 2012; Cohen et al. 2013; De Storme et al. 2013; Marinho et al. 2014; Rotreklová and Krahulcová 2016). Huber (1988) reported significant differences in diameter between pollen of di- and tetraploids in $R$. kuepferi, with diploid pollen being on average smaller than tetraploid pollen. Our microscopic investigations and results of a high-throughput pollen sizing method, measuring pollen diameters of several thousand pollen grains per individual volumetrically, in fact revealed a greater variation in size and shape than previously thought. Pollen of both cytotypes consisted of grains assignable to four distinctive size classes, found in pollen samples of every single individual. In accordance with the results of Huber (1988), most pollen grains in tetraploids have a slightly larger mean diameter (size classes $B_{1} C_{1}$ and $C_{2}$; see Table 2), when compared to pollen grains isolated from diploid plants in the same size ranges. Discernible and significant differences between pollen from di- and tetraploids occur mainly in proportions of observed pollen in one size class or another. Considering the differences in the distribution of the counted pollen among the different size classes as a whole, it is particularly striking that most pollen grains of tetraploids (53.0\%) were found in the smaller size class B (see Table 2, Fig. 3). Our microscopic studies generally confirmed these findings and additionally identified the smaller size classes as non-viable. Shriveled, misshapen and very small pollen grains (size class A), as well as normally shaped pollen grains with a diameter under $27 \mu \mathrm{m}$ (size class B), were not stainable, which accounts for more than two-third of all measured pollen in tetraploids. The unusual round and smooth shape of very large pollen (size class $C_{2}$ ) suggests that these might be as 
well infertile even though they contain high levels of starch, as shown by Lugol's iodine staining. Likely, only pollen grains with a diameter in the size range between 27 and $33 \mu \mathrm{m}$ (size class $C_{1}$ ) seem to represent fully developed, mature, viable pollen that can contribute to successful fertilization. A huge amount of aborted pollen grains and a highly variable pollen size as found in R. kuepferi is typical for facultatively apomictic plants (Izmailow 1996; Hörandl et al. 1997; Voigt et al. 2007) and likely results from meiotic and developmental disturbances during male gametophyte formation (e.g., Hojsgaard et al. 2014).

The observed differences in the proportions of size classes between the volumetric measurements of the Multisizer 3 and those detected by microscopy might be explained by the much larger number of pollen counts within samples that were automatically measured and/or by biases through subjective preference of measuring well-formed pollen during direct visual observation. Very big and especially very small particles further contribute to the fractions of size class $A$ and $C_{2}$, where highest deviations between measurements were found. Hence, the high-throughput method of volume-based particle size measurements allows for an accurate quantification of pollen size analysis, but is more informative if combined with the time-consuming method of direct optical examination by microscope, at least in our model system.

Because of the heterogeneity of pollen size and the overlap of ranges in diameter of viable pollen between diploid and tetraploids, we cannot directly infer pollen ploidy from pollen size in R. kuepferi. Hence, the assessment of actual frequencies and effects of unreduced and aneuploid male gamete formation still needs further investigation. Considering our flow cytometric seed screening results compared to our volumetric and microscopic pollen data in conjunction with the constraints of endosperm balance discussed above, we conclude that unreduced pollen are most likely only involved in rare cases of biparental sexual polyploidization, i.e., when the female gametes are unreduced too. 


\section{d) Mechanisms of unreduced gamete formation}

Although we observed female and male gamete formations only indirectly, some tentative conclusions can be drawn on the mechanisms of meiotic non-reduction. We suppose that apospory rather than restitutional meiosis is the major pathway to polyploidization in $R$. kuepferi. The great variation of pollen size classes and the regular occurrence of aneuploidy in female gametophytes of tetraploid plants (Table 1) suggest that unbalanced meiosis happens frequently in both male and female developments. The resulting micro- and megaspores have various unbalanced chromosome complements. During apomictic female gametophyte development, unreduced embryo sac formation happens in R. kuepferi via apospory (Burnier et al. 2009), which is also well documented for other Ranunculus species (Nogler 1984i Hojsgaard et al. 2014). Diplospory, as a result of female restitutional meiosis, has never been reported for Ranunculus. In aposporous $2 n$ megaspore formation, a somatic cell of the nucellus takes over gametophyte identity and development, while meiotic products abort. This way, female gametophyte development bypasses all detrimental effects of disturbed chromosomal segregation at meiosis and instead produces megaspores with complete unreduced chromosome sets. For male reproductive development, no alternative to meiosis is available, and reduced, unreduced and unbalanced pollen is probably produced in parallel. Selection on euploid male gametes and constraints of endosperm balance will probably favor the involvement of normally reduced pollen grains (i.e., haploid) for fertilization and final seed development, while unreduced pollen appear to be rarely successful.

\section{e) Implications for the evolution of polyploidy and apomixis}

Our data suggest that polyploidization in $R$. kuepferi mainly occurs through female triploid bridge, as unreduced female gamete formation was observed in all cytotypes. This way, triploids can easily be generated, and in turn produce tetraploid offspring (Fig. 6). The pathway of a male triploid bridge was not observed in this species. Since the capability to form unreduced female gametophytes via apospory is supposed to be a heritable trait (Nogler 1984i Ozias-Akins and Van Dijk 2007), tetraploids that originated from the described female variant of unilateral sexual polyploidization should be able to inherit this trait to the offspring. Continued fertilization and $\mathrm{B}_{\| I}$ offspring formation would result in constant increase in ploidy lev- 
els, which is, however, limited by cellular constraints (Comai 2005). In contrast, if regular unreduced embryo sac formation is coupled to parthenogenetic development, then the plants can keep ploidy levels constant in the offspring and reproduce fully asexually via seeds (apomixis). Such a fully functional apomixis is the predominant mode of reproduction in tetraploid R. kuepferi populations (Schinkel et al. 2016). Strikingly, fully functional apomixis (with unreduced, unfertilized egg cells) has already been observed in some diploid populations (Schinkel et al. 2016), further confirming a general capacity and genetic determination for unreduced female gamete formation. Among tetraploids, these previous studies on $R$. kuepferi did not detect a single tetraploid individual that shows full sexual reproduction, which would be expected if polyploidization is caused by unreduced pollen as unilateral, sexval and male-dependent process. Based on the absence of $3 x$ seeds resulting from male $2 n$ gametes, we thus assume that sexual tetraploid populations probably never originated in $R$. kuepferi.

a

1st step:

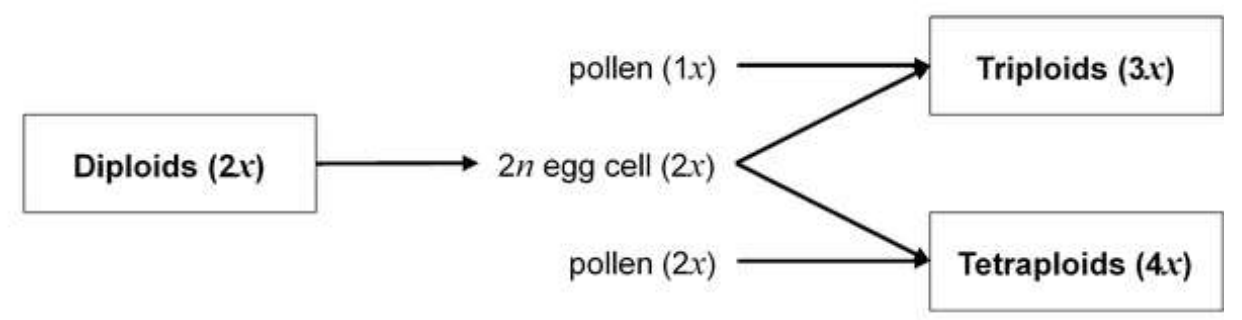

b

2nd step:

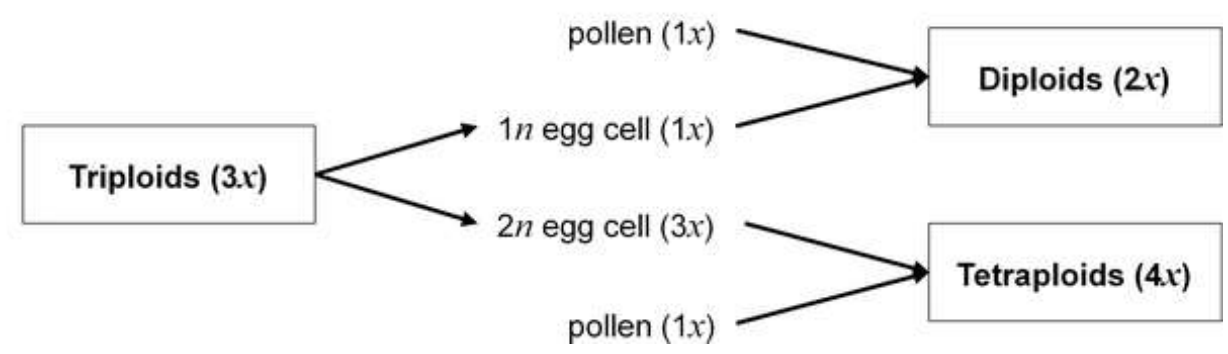

Fig. 6 Scheme representing different observed pathways of evolutionary origin of polyploids among Ranunculus kuepferi. Triploid formation is depicted as first step of a female triploid bridge in a, tetraploid formation as second step in $\mathbf{b}$

The form of unreduced egg cell formation via apospory not only facilitates polyploidization but additionally aids in the establishment of the apomictic mode of reproduction. The conclusion that partial apomixis is rather cause than consequence of polyploidy contradicts older theories which postulated polyploidy to be a precondition for expression of apomixis (Nogler 
1984; Carman 1997; Koltunow and Grossniklaus 2003). According to our results, it is rather the other way round. First, apospory appears in diploids, resulting in polyploidization. Then, after coupling to parthenogenesis, apomixis would be established in the polyploid line. These findings also support the idea of apomixis being a springboard for polyploidization and further evolution rather than a dead end (Hörandl and Hojsgaard 2012). The coupling of unreduced egg cell formation to parthenogenesis has further implications for the establishment of newly formed tetraploids. Sexual neopolyploids often suffer from a minority cytotype disadvantage (Levin 1975), as they emerge as single or a few individuals in a predominantly diploid population, where they would be fertilized predominantly with "wrong" haploid pollen. Triploid embryo formation and the above-discussed endosperm imbalances would lower their fitness and impede establishment of polyploids. In contrast, the shift to apomixis and self-fertility can overcome this problem and allow for establishment of polyploid populations even via a single or a few tetraploid individuals. This advantage of tetraploid apomictic populations, among others, may have contributed to the observed large distributions of tetraploid R. kuepferi, in contrast to sexual diploid populations which failed to expand their geographical area after the last glaciation (Schinkel et al. 2016; Kirchheimer et al. 2016). 


\section{References}

Aliyu OM, Schranz ME and Sharbel TF. 2010. Quantitative variation for apomictic reproduction in the genus Boechera (Brassicaceae). American Journal of Botany 97:1719-1731

Baack EJ. 2005. Ecological factors influencing tetraploid, establishment in snow buttercups (Ranunculus adoneus, Ranunculaceae): Minority cytotype exclusion and barriers to triploid formation. American Journal of Botany 92:1827-1835

Bell G. 1982. The Masterpiece of Nature: The Evolution and Genetics of Sexuality. Cambridge University Press Archive, Cambridge

Bicknell RA and Koltunow AM. 2004. Understanding Apomixis: Recent Advances and Remaining Conundrums. The Plant Cell 16:228-245

Bicknell RA, Lambie SC and Butler RC. 2003. Quantification of progeny classes in two facultatively apomictic accessions of Hieracium. Hereditas 138:11-20

Bierzychudek P. 1985. Patterns in plant parthenogenesis. Experientia 41:1255-1264

Blanc G, Hokamp K and Wolfe KH. 2003. A recent polyploidy superimposed on older large-scale duplications in the Arabidopsis genome. Genome Research 13:137-144

Bretagnolle F and Thompson JD. 1995. Gametes with the somatic chromosome number: mechanisms of their formation and role in the evolution of autopolypoid plants. New Phytologist 129:1-22

Brink RA and Cooper DC. 1947. The endosperm in seed development. The Botanical Review 13:479-541

Brownfield L and Köhler C. 2011. Unreduced gamete formation in plants: mechanisms and prospects. Journal of Experimental Botany 62:1659-1668

Burgess MB, Cushman KR, Doucette ET, Talent N, Frye CT and Campbell CS. 2014. Effects of apomixis and polyploidy on diversification and geographic distribution in Amelanchier (Rosaceae). American Journal of Botany 101:1375-1387

Burnier J, Buerki S, Arrigo N, Küpfer P and Alvarez N. 2009. Genetic structure and evolution of Alpine polyploid complexes: Ranunculus kuepferi (Ranunculaceae) as a case study. Molecular Ecology 18:3730-3744

Carman JG. 1997. Asynchronous expression of duplicate genes in angiosperms may cause apomixis, bispory, tetraspory, and polyembryony. Biological Journal of the Linnean Society 61:51-94

Cohen H, Fait A and Tel-Zur N. 2013. Morphological, cytological and metabolic consequences of autopolyploidization in Hylocereus (Cactaceae) species. BioMed Central Plant Biology 13:173-186

Comai L. 2005. The advantages and disadvantages of being polyploid. Nature Reviews Genetics 6:836-846

Cosendai A-C and Hörandl E. 2010. Cytotype stability, facultative apomixis and Geographical Parthenogenesis in Ranunculus kuepferi (Ranunculaceae). Annals of Botany 105:457-470

Cosendai A-C, Rodewald J and HörandI E. 2011. Origin and distribution of autopolyploids via apomixis in the alpine species Ranunculus kuepferi (Ranunculaceae). Taxon 60:355-364 
Cosendai A-C, Wagner J, Ladinig U, Rosche C and Hörandl E. 2013. Geographical parthenogenesis and population genetic structure in the alpine species Ranunculus kuepferi (Ranunculaceae). Heredity 110:560-569

De Storme N and Geelen D. 2013. Sexual Polyploidization in Plants: Cytological Mechanisms and Molecular Regulation. New Phytologist 198:670-684

De Storme N, Copenhaver GP and Geelen D. 2012. Production of diploid male gametes in Arabidopsis by cold-induced destabilization of postmeiotic radial microtubule arrays. Plant Physiology 160:18081826

De Storme N, Zamariola L, Mau M, Sharbel TF and Geelen D. 2013. Volume-based pollen size analysis: an advanced method to assess somatic and gametophytic ploidy in flowering plants. Plant Reproduction 26:65-81

Dobeš C, Lückl A, Hülber K and Paule J. 2013. Prospects and limits of the flow cytometric seed screen insights from Potentilla sensu lato (Potentilleae, Rosaceae). New Phytologist 198:605-616

Dobeš C, Mitchell-Olds T and Koch M. 2004. Extensive chloroplast haplotype variation indicates Pleistocene hybridization and radiation of North American Arabis drummondii, A. x divaricarpa, and A. holboellii (Brassicaceae). Molecular Ecology 13:349-370

Doležel J, Greilhuber J and Suda J. 2007. Estimation of nuclear DNA content in plants using flow cytometry. Nature Protocols 2:2233-2244

Glennon KL, Ritchie ME and Segraves KA. 2014. Evidence for shared broad-scale climatic niches of diploid and polyploid plants. Ecology Letters 17:574-582

Greilhuber J, Doležel J, Lysák MA and Bennett MD. 2005. The origin, evolution and proposed stabilization of the terms 'Genome Size' and 'C-Value' to describe nuclear DNA contents. Annals of Botany 95:255-260

Haig D. 2013. Kin conflict in seed development: An interdependent but fractious collective. Annual Review of Cell and Developmental Biology 29:189-211

Haig D and Westoby M. 1991. Genomic imprinting in the endosperm: its effect on seed development in crosses between species, and between different ploidies of the same species, and its implications for the evolution of apomixis. Philosophical Transactions of The Royal Society B: Biological Sciences 333:1-13

Harlan JR and de Wet JMJ. 1975. On Ö. Winge and a prayer: the origins of polyploidy. The Botanical Review 41:361-390

Hegarty MJ and Hiscock SJ. 2008. Genomic clues to the evolutionary success of polyploid plants. Current Biology 18:435-444

Hojsgaard D, Greilhuber J, Pellino M, Paun O, Sharbel TF and Hörandl E. 2014. Emergence of apospory and bypass of meiosis via apomixis after sexual hybridisation and polyploidisation. New Phytologist 204:1000-1012 
Hojsgaard D, Martínez EJ and Quarin CL. 2013. Competition between meiotic and apomictic pathways during ovule and seed development results in clonality. New Phytologist 197:336-347

Hörandl E. 2006. The complex causality of Geographical Parthenogenesis. New Phytologist 171(3):525-538 Hörandl E and Hojsgaard D. 2012. The evolution of apomixis in angiosperms: a reappraisal. Plant Biosystems 146(3):681-693

Hörandl E and Temsch EM. 2009. Introgression of apomixis into sexual species is inhibited by mentor effects and ploidy barriers in the Ranunculus auricomus complex. Annals of Botany 104:81-89

HörandI E, Cosendai A-C and Temsch EM. 2008. Understanding the geographic distributions of apomictic plants: a case for a pluralistic approach. Plant Ecology and Diversity 1:309-320

Hörandl E, Dobeš CH and Lambrou M. 1997. Chromosomen- und Pollenuntersuchungen an österreichischen Sippen des Ranunculus auricomus-Komplexes. Botanica Helvetica 107:195-209

Huber W. 1988. Natürliche Bastardierungen zwischen weissblühenden Ranunculus-Arten in den Alpen [Natural hybridizations between white-flowered species of Ranunculus in the Alps]. Veröffentlichungen des Geobotanischen Institutes der Eidgenössischen Technischen Hochschule, Stiftung Rübel, Zürich 100:1-160

Hull-Sanders HM, Johnson RH, Owen HA and Meyer GA. 2009. Effects of polyploidy on secondary chemistry, physiology, and performance of native and invasive genotypes of Solidago gigantea (Asteraceae). American Journal of Botany 96:762-770

Husband BC. 2000. Constraints on polyploid evolution: a test of the minority cytotype exclusion principle. Proceedings of the Royal Society B: Biological Sciences 267:217-223

Husband BC. 2004. The role of triploid hybrids in the evolutionary dynamics of mixed-ploidy populations. Biological Journal of the Linnean Society 82:537-546

Husband BC and Schemske DW. 2000. Ecological mechanisms of reproductive isolation and coexistence of diploid and tetraploid Chamerion angustifolium. Journal of Ecology 88:1-14

Izmailow R. 1996. Reproductive strategy in the Ranunculus auricomus complex (Ranunculaceae). Acta Societatis Botanicorum Poloniae 65:167-170

Jiao Y, Wickett NJ, Ayyampalayam S, Chanderbali AS, Landherr L, Ralph PE, Tomsho LP, Hu Y, Liang $H$, Soltis PS, Soltis DE, Clifton SW, Schlarbaum SE, Schuster SC, Ma H, Leebens-Mack J and de Pamphilis CW. 2011. Ancestral polyploidy in seed plants and angiosperms. Nature 473:97-100

Karpechenko GD. 1927. The production of polyploid gametes in hybrids. Hereditas 9:349-368

Kearney M. 2005. Hybridization, glaciation and Geographical Parthenogenesis. Trends in Ecology \& Evolution 20:495-502

Keller F, Körner C. 2003. The role of photoperiodism in alpine plant development. Arctic, Antarctic, and Alpine Research 35:361-368

Kirchheimer B, Schinkel CCF, Dellinger AS, Klatt S, Moser D, Winkler M, Lenoir J, Caccianiga M, Guisan A, Nieto-Lugilde D, Svenning J-C, Thuiller W, Vittoz P, Willner W, Zimmermann NE, 
Hörandl E and Dullinger S. 2016. A matter of scale: apparent niche differentiation of diploid and tetraploid plants may depend on extent and grain of analysis. Journal of Biogeography 43:716-726

Klatt S, Hadacek F, Hodač L, Brinkmann G, Eilerts M, Hojsgaard D and Hörandl E. 2016. Photoperiod extension enhances sexual megaspore formation and triggers metabolic reprogramming in facultative apomictic Ranunculus auricomus. Frontiers in Plant Science 7:278-293

Köhler C, Mittelsten-Scheid O and Erilova A. 2010. The impact of the triploid block on the origin and evolution of polyploid plants. Trends in Genetics 26:142-148

Koltunow AM and Grossniklaus U. 2003. Apomixis: a developmental perspective. Annual Review of Plant Biology 54:547-574

Kurepin LV, Walton LJ, Reid DM, Chinnappa CC and Pharis RP. 2007. Photoperiod, light quality, and irradiance effects on flowering in the alpine and prairie ecotypes of Stellaria longipes. Canadian Journal of Botany 85:538-544

Levin DA. 1975. Minority cytotype exclusion in local plant populations. Taxon 24:35-43

Levin DA. 1983. Polyploidy and novelty in flowering plants. The American Naturalist 122:1-25

Levin DA. 2004. The ecological transition in speciation. New Phytologist 161:91-96

Lin B-Y. 1984. Ploidy barrier to endosperm development in Maize. Genetics 107:103-15

Lokhande SD, Ogawa K, Tanaka A and Hara T. 2003. Effect of temperature on ascorbate peroxidase activity and flowering of Arabidopsis thaliana ecotypes under different light conditions. Journal of Plant Physiology 160:57-64

Lovell JT, Aliyu OM, Mau M, Schranz ME, Koch M, Kiefer C, Song B-H, Mitchell-Olds T and Sharbel TF. 2013. On the origin and evolution of apomixis in Boechera. Plant Reproduction 26:309-315

Lu J, Zhang C, Baulcombe DC and Chen ZJ. 2012. Maternal siRNAs as regulators of parental genome imbalance and gene expression in endosperm of Arabidopsis seeds. Proceedings of the National Academy of Sciences of the United States of America. 109:5529-5534

Ludwig S, Robertson A, Rich TCG, Djordjevic M, Cerovic R, Houston L, Harris SA and Hiscock SJ. 2013. Breeding systems, hybridization and continuing evolution in Avon Gorge Sorbus. Annals of Botany 111:563-575

Marinho RC, Mendes-Rodrigues C, Bonetti AM and Oliveira PE. 2014. Pollen and stomata morphometrics and polyploidy in Eriotheca (Malvaceae-Bombacoideae). BMC Plant Biology 16:508-511

Mason AS and Pires JC. 2015. Unreduced gametes: meiotic mishap or evolutionary mechanism?. Trends in Genetics 31:5-10

Masterson J. 1994. Stomatal size in fossil plants: evidence for polyploidy in majority of angiosperms. Science 264:421-24

Matzk F, Meister A and Schubert I. 2000. An efficient screen for reproductive pathways using mature seeds of monocots and dicots. The Plant Journal 21:97-108

Mirzaghaderi G, Hörandl E. 2016. The evolution of meiotic sex and its alternatives. Proceedings of the Royal Society B: Biological Sciences 283:20161221 
Nogler GA. 1984. Gametophytic apomixis. In: Johri PBM (eds) Embryology of angiosperms. Springer, Berlin, $p p$ 475-518

Otto SP and Whitton J. 2000. Polyploid Incidence and Evolution. Annual Review of Genetics 34:401-437

Ozias-Akins P and Van Dijk PJ. 2007. Mendelian genetics of apomixis in plants. Annual Review of Genetics 41:509-537

Parisod C and Besnard G. 2007. Glacial in situ survival in the Western Alps and polytopic autopolyploidy in Biscutella laevigata L. (Brassicaceae). Molecular Ecology 16:2755-2767

Pecrix Y, Rallo G, Folzer H, Cigna M, Gudin S and Le Bris M. 2011. Polyploidization mechanisms: temperature environment can induce diploid gamete formation in Rosa sp. Journal of Experimental Botany 62:3587-3597

Quarin CL. 1986. Seasonal changes in the incidence of apomixis of diploid, triploid, and tetraploid plants of Paspalum cromyorrhizon. Euphytica 35:515-522

R Core Team. 2014. R: A language and environment for statistical computing. $R$ Foundation for Statistical Computing

Ramsey J and Schemske DW. 1998. Pathways, mechanisms, and rates of polyploid formation in flowering plants. Annual Review of Ecology and Systematics 29:467-501

Ramsey J and Schemske DW. 2002. Neopolyploidy in flowering plants. Annual Review of Ecology and Systematics 33:589-639

Rani S, Gupta RC, Kumari S, Rana JC. 2013. Chromosomal diversity in three species of genus Rosa L. (Rosaceae) from district Kangara of Himachal Pradesh. Indian Journal of Genetics and Plant Breeding 73:454-458

Randle AM, Slyder JB and Kalisz S. 2009. Can differences in autonomous selfing ability explain differences in range size among sister taxa pairs of Collinsia (Plantaginaceae)? An extension of Baker's Law. New Phytologist 183:618-629

Róis AS, Teixeira G, Sharbel TF, Fuchs J, Martins S, Espírito-Santo D and Caperta AD. 2012. Male fertility versus sterility, cytotype, and DNA quantitative variation in seed production in diploid and tetraploid sea lavenders (Limonium sp., Plumbaginaceae) reveal diversity in reproduction modes. Sexual Plant Reproduction 25:305-318

Rotreklová O and Krahulcová A. 2016. Estimating paternal efficiency in an agamic polyploid complex: pollen stainability and variation in pollen size related to reproduction mode, ploidy level and hybridogenous origin in Pilosella (Asteraceae). Folia Geobotanica 51:175-186

Šarhanová $P$, Vašut RJ, Dančák $M$, Bureš $P$ and Trávniček $B$. 2012. New insights into the variability of reproduction modes in European populations of Rubus subgen. Rubus: how sexual are polyploid brambles?. Sexual Plant Reproduction 25:319-335

Schinkel CCF, Kirchheimer B, Dellinger AS, Klatt S, Winkler M, Dullinger S and Hörandl E. 2016. Correlations of polyploidy and apomixis with elevation and associated environmental gradients in an alpine plant. AoB PLANTS 8:plwo64 
Schranz ME, Dobeš C, Koch MA and Mitchell-Olds T. 2005. Sexual reproduction, hybridization, apomixis, and polyploidization in the genus Boechera (Brassicaceae). American Journal of Botany 92:17971810

Scott RJ. 2007. Polyspermy in apomictic Crataegus: yes and no. New Phytologist 173:227-230

Segraves KA, Thompson JN, Soltis PS and Soltis DE. 1999. Multiple origins of polyploidy and the geographic structure of Heuchera grossulariifolia. Molecular Ecology 8:253-262

Sharbel TF, Voigt ML, Corral JM, Thiel T, Varshney A, Kumlehn J, Vogel H and Rotter B. 2009. Molecular signatures of apomictic and sexual ovules in the Boechera holboellii complex. Plant Journal 58:870-882

Soltis DE and Soltis PS. 1999. Polyploidy: recurrent formation and genome evolution. Trends in Ecology \& Evolution 14:348-352

Soltis DE and Soltis PS. 2000. Contributions of plant molecular systematics to studies of molecular evolution. Plant Molecular Biology Reporter 42:45-75

Soltis DE and Soltis PS. 2009. The role of hybridization in plant speciation. Annual Review of Plant Biology $60: 561-588$

Soltis DE, Soltis PS, Pires JC, Kovarik A, Tate JA and Mavrodiev E. 2004. Recent and recurrent polyploidy in Tragopogon (Asteraceae): cytogenetic, genomic and genetic comparisons. Biological Journal of the Linnean Society 82:485-501

Sora D, Kron P, Husband BC. 2016. Genetic and environmental determinants of unreduced gamete production in Brassica napus, Sinapis arvensis and their hybrids. Heredity 117:440-448

Spielmann M, Vinkenoog R and Scott RJ. 2003. Genetic mechanisms of apomixis. Philosophical Transactions of The Royal Society B: Biological Sciences 358:1095-1103

Stebbins GL. 1950. Variation and Evolution in Plants. Columbia University Press, New York

Stebbins GL. 1971. Chromosomal Evolution in Higher Plants. Addison-Wesley, London

Strasburger E. 1910. Sexuelle und apogame Fortpflanzung bei Urticaceen. Jahrbuch der Wissenschaftlichen Botanik 47:245-288

Talent $\mathbf{N}$ and Dickinson TA. 2007. Endosperm formation in aposporous Crataegus (Rosaceae, Spiraeoideae, tribe Pyraceae): parallels to Ranunculaceae and Poaceae. New Phytologist 173:231-249

Tayale A and Parisod C. 2013. Natural pathways to polyploidy in plants and consequences for genome reorganization. Cytogenetic and Genome Research 140:79-96

Tie S, Xia J, Qiu F and Zheng Y. 2006. Genome-wide analysis of maize cytoplasmic male sterility-S based on QTL mapping. Plant Molecular Biology Reporter 24:71-80

Treier UA, Broennimann O, Normand S, Guisan A, Schaffner U, Steinger T and Müller-Schärer H. 2009. Shift in cytotype frequency and niche space in the invasive plant Centaurea maculosa. Ecology 90:1366-1377

Van Dijk PJ. 2003. Ecological and evolutionary opportunities of apomixis: insights from Taraxacum and Chondrilla. Philosophical Transactions of The Royal Society B: Biological Sciences 358:1113-1121 
Vinkenoog R, Bushell C, Spielman M, Adams S, Dickinson HG and Scott RJ. 2003. Genomic imprinting and endosperm development in flowering plants. Molecular Biotechnology 25:149-184

Voigt ML, Melzer M, Rutten T, Mitchell-Olds T and Sharbel TF. 2007. Gametogenesis in the apomictic Boechera holboellii complex: the male perspective. In: Hörandl E, Grossniklaus U, Van Dijk PJ, Sharbel T (eds) Apomixis: evolution, mechanisms and perspective. Gantner, Ruggell, pp 235-258

Wang Y-Q, Zhang D-X and Chen Z-Y. 2004. Pollen Histochemistry and Pollen: Ovule Ratios in Zingiberaceae. Annals of Botany $94: 583-591$

Wang X, Cheng Z-M, Zhi S, Xu F. 2016. Breeding Triploid Plants: A Review. Czech Journal of Genetics and Plant Breeding 52:41-54

Wendel JF. 2000. Genome evolution in polyploids. Plant Molecular Biology Reporter 42:225-249

Wood TE, Takebayashi N, Barker MS, Mayrose I, Greenspoon PB and Rieseberg LH. 2009. The frequency of polyploid speciation in vascular plants. Proceedings of the National Academy of Sciences of the United States of America. 106:13875-13879 


\section{Supplementary Material}

Online Resource 1. Table of flow cytometry data of all individuals with peak position on flow histograms and total number of seeds.

\begin{tabular}{|c|c|c|c|c|c|c|c|}
\hline \multirow[b]{2}{*}{ Individual } & \multirow[b]{2}{*}{ Ploidy } & \multicolumn{3}{|l|}{ Embryo } & \multicolumn{3}{|c|}{ Endosperm } \\
\hline & & Position & CV [\%] & $\mathrm{N}$ & Position & CV [\%] & $\mathrm{N}$ \\
\hline $31-3-2$ & $2 x$ & 155.8 & 3.8 & 127 & 251.8 & $4 \cdot 4$ & 1808 \\
\hline 204-1-1 & $2 x$ & 192.6 & 4.8 & 178 & 279.6 & 4.7 & 1073 \\
\hline $204-1-1$ & $2 x$ & 146.9 & $5 \cdot 5$ & 232 & 242.0 & 6.5 & 692 \\
\hline $204-1-2$ & $2 x$ & 152.0 & 4.9 & 155 & 239.8 & 6.4 & 1279 \\
\hline $200-1-2$ & $3 x$ & 190.3 & 4.0 & 239 & 515.6 & $5 \cdot 2$ & 913 \\
\hline $204-3-2$ & $3 x$ & 93.1 & 5.2 & 219 & 303.7 & $4 \cdot 3$ & 1349 \\
\hline $204-3-2$ & $3 x$ & 185.2 & 4.2 & 201 & $457 \cdot 4$ & 6.8 & 1222 \\
\hline $204-3-2$ & $3 x$ & 190.1 & 4.1 & 230 & 332.0 & 4.8 & 867 \\
\hline $204-3-3$ & $3 x$ & 92.2 & 4.8 & 232 & 300.1 & $5 \cdot 3$ & 1497 \\
\hline $205-2-1$ & $3 x$ & 195.2 & $5 \cdot 2$ & 226 & 340.4 & $5 \cdot 5$ & 978 \\
\hline $36-4-2$ & $4 x$ & 292.7 & $4 \cdot 5$ & 164 & 485.9 & 4.1 & 523 \\
\hline $37-3-2$ & $4 x$ & 301.4 & $5 \cdot 5$ & 213 & 493.5 & 4.0 & 770 \\
\hline $36-1-2$ & $4 x$ & 93.1 & 5.6 & 204 & $283 \cdot 3$ & 4.0 & 1093 \\
\hline $36-1-2$ & $4 x$ & 143.7 & 3.1 & 93 & 373.5 & 5.2 & 419 \\
\hline $40-4-3$ & $4 x$ & 282.9 & 2.3 & 117 & 674.4 & 3.1 & 1166 \\
\hline $36-1-2$ & $4 x$ & 285.6 & 3.8 & 181 & 464.6 & 3.6 & 592 \\
\hline $36-1-1$ & $4 x$ & 298.1 & 3.2 & 126 & 485.8 & 4.0 & 310 \\
\hline $40-4-2$ & $4 x$ & 306.6 & 2.6 & 86 & 500.8 & 4.2 & 1566 \\
\hline $45^{-1-1}$ & $4 x$ & 300.3 & 2.5 & 84 & 492.7 & 3.9 & 569 \\
\hline $54-2-1$ & $4 x$ & 289.2 & $4 \cdot 3$ & 145 & 482.4 & 4.0 & 408 \\
\hline $58-2-3$ & $4 x$ & 312.6 & 2.6 & 210 & 515.7 & 3.8 & 875 \\
\hline $58-2-2$ & $4 x$ & 312.8 & 3.1 & 147 & $539 \cdot 5$ & 3.6 & 705 \\
\hline $73-1-3$ & $4 x$ & 289.4 & 1.7 & 104 & 480.4 & 2.5 & 1128 \\
\hline $111-1-2$ & $4 x$ & 106.2 & 2.8 & 203 & 306.4 & 2.5 & 827 \\
\hline $200-2-3$ & $4 x$ & 93.1 & $7 \cdot 3$ & 347 & 278.3 & $5 \cdot 7$ & 954 \\
\hline $200-2-3$ & $4 x$ & 93.8 & 7.1 & 416 & 276.6 & $4 \cdot 9$ & 674 \\
\hline $200-2-3$ & $4 x$ & 94.9 & 6.7 & 416 & 278.9 & $5 \cdot 7$ & 560 \\
\hline $200-2-2$ & $4 x$ & 95.9 & $5 \cdot 9$ & 374 & 283.8 & 4.8 & 1100 \\
\hline
\end{tabular}




\begin{tabular}{llllllll}
$200-1-3$ & $4 x$ & 96.7 & 5.2 & 459 & 284.5 & 5.0 & 1300 \\
$200-2-2$ & $4 x$ & 96.9 & 6.7 & 338 & 289.2 & 4.0 & 1039 \\
$200-1-3$ & $4 x$ & 99.3 & 6.0 & 374 & 295.6 & 5.9 & 802 \\
$200-1-3$ & $4 x$ & 101.2 & 6.2 & 396 & 297.3 & 4.5 & 1073 \\
$204-2-3$ & $4 x$ & 100.6 & 6.2 & 236 & 247.4 & 6.2 & 1067 \\
$204-2-3$ & $4 x$ & 144.8 & 4.8 & 166 & 279.9 & 5.6 & 937 \\
$205-4-2$ & $4 x$ & 139.0 & 5.2 & 213 & 347.6 & 5.4 & 829 \\
$204-3-1$ & $4 x$ & 96.4 & 5.1 & 261 & 280.1 & 4.9 & 1381 \\
$204-3-1$ & $4 x$ & 149.1 & 4.6 & 174 & 387.9 & 5.0 & 831 \\
$204-3-1$ & $4 x$ & 152.1 & 4.0 & 194 & 394.4 & 4.4 & 1211 \\
$204-3-1$ & $4 x$ & 152.6 & 4.0 & 171 & 394.3 & 4.4 & 1126 \\
$205-4-1$ & $4 x$ & 147.2 & 4.9 & 205 & 335.5 & 6.6 & 863 \\
$205-4-1$ & $4 x$ & 149.0 & 5.2 & 1037 & 244.6 & 5.1 & 390 \\
$205-4-1$ & $4 x$ & 145.4 & 5.8 & 252 & 370.3 & 5.5 & 520 \\
$205-4-1$ & $4 x$ & 147.6 & 4.6 & 378 & 382.7 & 4.7 & 799 \\
\hline
\end{tabular}

Online Resource 2. Diagram of time series analyses of pollen diameter measures after different incubation times on randomly chosen pollen samples.

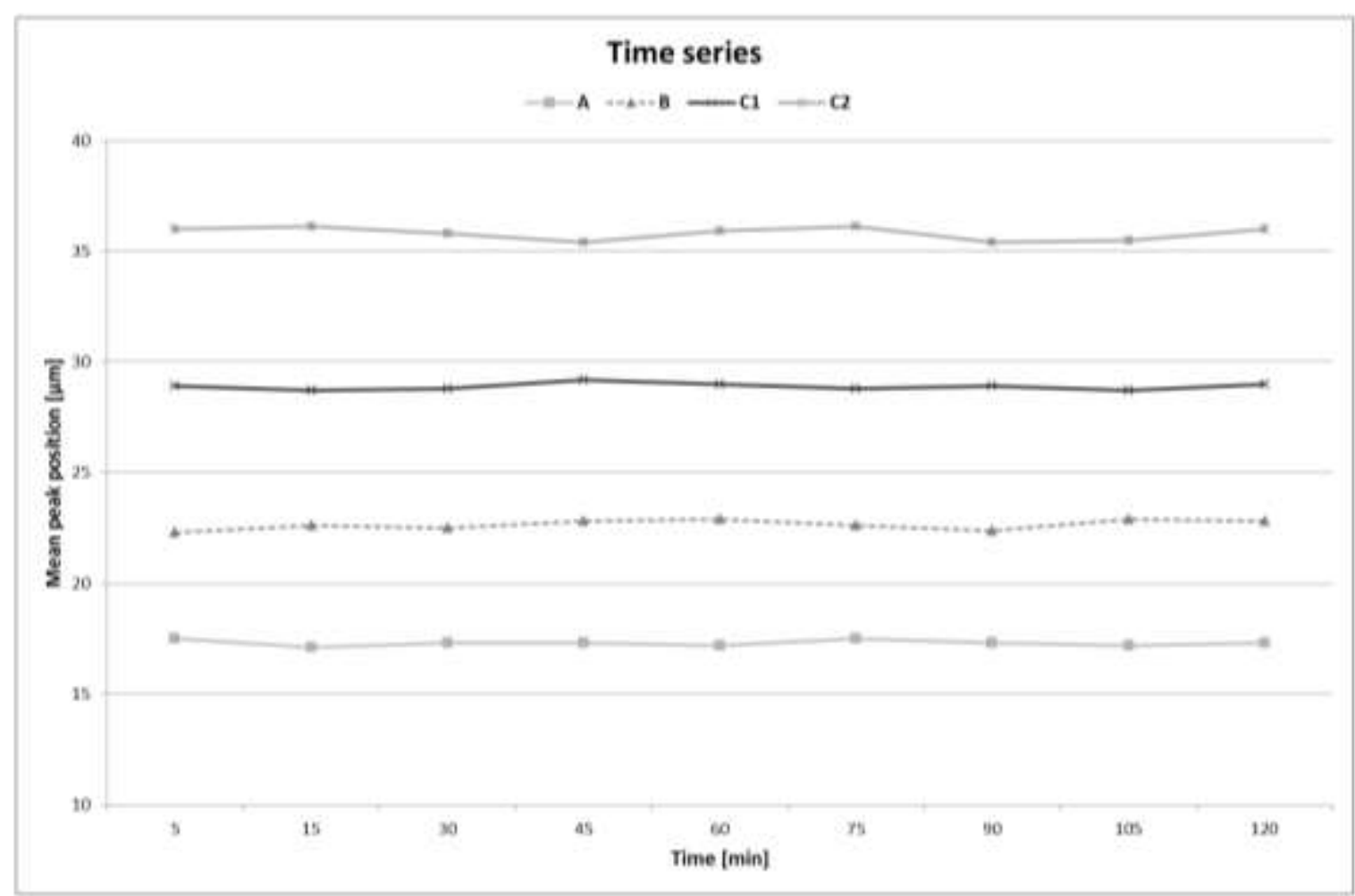




\section{Chapter 3}

Epigenetic patterns and Geographical Parthenogenesis in the alpine species Ranunculus kuepferi (Ranunculaceae)

Christoph C. F. Schinkel, Bernhard Kirchheimer, Stefan Dullinger, Simone Klatt, Eleni

Syngelaki and Elvira Hörandl

Manuscript Draft 


\section{Abstract}

Epigenetic mechanism are important regulators of the response of plants to environmental conditions. Specifically, cytosin-methylation as heritable epigenetic modification is thought to have an important transgenerational effect on adaptation. Polyploidization and the switch to apomictic reproduction are correlated to changes of specific methylation profiles. We therefore hypothesize that DNA methylation plays a key role for the expression of apomixis, as well as the adaptation of plants to alpine environments. Both factors have contributed to the larger distribution areas of apomictic polyploids (Geographical Parthenogenesis).

On our alpine model system Ranunculus kuepferi, we tested differences in methylation patterns and their correlation to environmental data and geographical distributions across two cytotypes (diploid, tetraploid) and three reproduction modes (sexual, mixed, apomictic), by Methylation-sensitive Amplified Fragment-Length Polymorphism (MSAP). We used the isoschizomer enzymes Msp1 (non-sensitive) and Hpaz (methylation-sensitive) combined with EcoR1.

MSAP profiles differed between both ploidy levels and the three modes of reproduction, both separately and between three combined groups ( $2 x$ sexual vs. $4 x$ apomictic and $4 \times$ mixed). The strongest differentiation was found for all types of epiloci between the tetraploid mixed and apomictic groups, suggesting an influence on expression of apomixis. The more conserved internally methylated epiloci correlate strongly to ploidy and may indicate partly heritable patterns. Although there was no specific spatial pattern detectable, all epiloci showed negative correlations to main climatic variables related to altitude. Correlation to climatic factors suggests an adaptive role of methylation in tetraploid apomictic $R$. kuepferi to harsh, cold conditions in the high alpine habitats. Cold induced epigenetic changes could have been a stimulus for the shift to apomixis.

\section{Introduction}

Apomixis refers to an asexual form of reproduction that produces clonally developed seeds as heritable trait (Nogler 1984, Koltunow \& Grossniklaus 2003, Ozias \& Van Dijk 2007). Unreduced embryo sacs are formed from either somatic nucellus cells or via restitutional meiosis during megaspore development (Asker \& Jerling 1992; Koltunow \& Grossniklaus 2003). Apomictic seeds sustain the genome configuration of their parental progenitors and hence establish the fixation of any genotype. Although apomixis is widespread in natural populations 
and occurs among at least 78 families and more than 290 genera of angiosperms (Hojsgaard et al. 2014), genetic control mechanisms on its expression still remain elusive. In general, apomixis appears to be triggered by a spatial or rather temporal deregulation of the genes that control the sexual meiotic development of female gametes (Koltunow \& Grossniklaus 2003, Ronceret \& Vielle-Calzada 2015). For example, imbalanced expression of genes in the AGO protein family are suspected to influence cell fate in the ovule and may provoke somatic cells to form a apomeiotic megaspore mother cell (e. g. Grimanelli 2012, Hand \& Koltunow 2014). By avoiding meiosis it has the potential to counteract negative distributional effects in chromosome segregation of recently established (de novo) polyploid species (Comai 2005).

Almost all natural apomictic plants are polyploid (Carman 1997). Polyploidy is a naturally occurring condition in which organisms possess more than two sets of chromosomes. It is an elementary component of evolution (Ramsey \& Schemske 1998), especially in plants (Soltis \& Soltis 2000, Wendel 2000) and plays an important role in adaptation to new and harsh environments as well as speciation processes (Levin 1983, Soltis \& Soltis 2009, Wood et al. 2009). Genome duplication is often accompanied by increased phenotypic plasticity (Hegarty and Hiscock 2008), which can result in evolutionary advantages. Furthermore Levin (1975, 2003) postulated that successful establishment of polyploid taxa involves niche differentiation, which is attended by shifted or even broadened tolerance spectra to environmental influences. Thereby a switch to asexual reproduction counteracts potential detrimental reproductive disadvantages. Hence, apomixis may be induced by or related to multiple genetic changes associated with the whole genome duplication/multiplication, such as transcriptional remodelling (Carman 1997, Otto \& Whitton 2000, Koltunow \& Grossniklaus 2003, Comai 2005, Cifuentes et al. 2009). However, recent studies suggest that apomixis can originate spontaneously in diploids (Bicknell 1997, Dobeš et al. 2006, Ozias-Akins \& van Dijk 2007, Kantama et al. 2007, Siena et al. 2008, Aliyu et al. 2010, Schinkel et al. 2016, Barke et al. 2018). Many studies have shown that apomictic polyploids frequently exhibit rapid range expansions which often result in larger distribution areas compared to their diploid progenitors, a pattern called Geographical Parthenogenesis (van Dijk 2003, Kearney 2005, Hörandl 2006, Hörandl et al. 2008, Hull-Sanders et al. 2009, Treier et al. 2009). Such range expansions of polyploids express a tendency to particularly higher latitudes as well as elevations and accordingly more extreme habitats, i. e. colder climates (Bell 1982, Bierzychudek 1985, Kearney 2005, Hörandl 2006, Gregor 2013, Schinkel et al. 2016). Natural spontaneous occurrences of 
apomixis have been particularly evident during climatic fluctuations throughout the Pleistocene (Carman 1997, Dobes et al. 2004, Paun et al. 2006). Experimental studies affirmed that abiotic factors such as light and especially temperature regime can shift the proportions between sexual and asexual reproduction in plants (Hörandl \& Hadacek 2013, Klatt et al. 2018). Exceedingly cold temperatures are known to cause restitutional meiosis and consequently generate unreduced gametes (Ramsey \& Schemske 1998, De Storme \& Geelen 2013, De Storme et al. 2013a, Bomblies et al. 2015). Unreduced female gamete formation is a major component of apomixis and at the same time a pathway to polyploidization (Schinkel et al. 2017). However, the genomic and epigenomic background for establishment of polyploid apomicts in colder climates is still largely unknown.

Epigenetic processes provide advanced molecular information additional to genetic regulatory mechanisms, which are subject to constant transformation that largely interact on phenotypic characteristics without altering DNA sequences (Richards 2006, Bird 2007, Verhoeven et al. 2016). Among a variety of such reversible modifications, methylation at the $5^{\prime}$ carbon of cytosine is an important epigenetic mechanism, particularly in plants (Jaenisch \& Bird 2003, Bender 2004). It affects individual development through control of gene regulation and expression, as well as cell differentiation by activating, reducing or completely disabling the activity of particular genomic segments (e.g. Grant-Downton \& Dickinson 2005, Berger 2007, Matzke et al. 2015, Espinas et al. 2016). Both biotic and abiotic environmental stimuli can trigger de novo and demethylation processes leading to different DNA methylation patterns (e.g. Labra et al. 2002, Sherman \& Talbert 2002, Dowen et al. 2012). Numerous other studies have demonstrated their effects on ecologically relevant traits and trait plasticity, that are frequently associated with the extent of genetic imprinting among heterochromatic and repetitive loci as well as silencing of both transposons and transgenes (Miura et al. 2001, Grossniklaus et al. 2001, Lippman et al. 2004, Zilberman et al. 2007, Jones 2012, 2014). Methylation is further involved in the regulatory mechanisms for expression of apomictic versus sexual reproduction (Shah et al. 2016; Rodrigo et al. 2017). It has also been demonstrated that genomic rearrangement itself due to hybridization or polyploidization can induce such changes in DNA methylation patterns (Adams \& Wendel 2005, Dong et al. 2006, GrantDownton \& Dickinson 2006). Furthermore it is known, that environmental stresses can induce veritable changes in the methylation patterns of plants. Epigenetic variation can cause more variable phenotypic plasticity and selectable heritable variation than genomic mixing 
(Herrera \& Bazaga 2013, Herman \& Sultan 2016, Banta \& Richards 2018, Ecker et al. 2018). In plants, particularly temperature seems to trigger certain methylation conversions (De Storme et al. 2012, De Storme \& Geelen 2013C, Verhoeven et al. 2010, Paun et al. 2010, Song et al. 2015, Banerjee et al. 2017, Liu et al. 2017). Such sensitivity could be associated with the potential to adapt rapidly to extreme environments. Since meiotic resetting of cytosine methylation is bypassed in apomictic plants, such lineages are also able to retain favorable methylation patterns over several generations. Previous experimental work on apomictic clonal dandelions demonstrated immediate response of plants to abiotic stress conditions, correlations to methylation patterns and heritability of traits with altered methylation profiles (Verhoeven et al. 2010; Verhoeven \& Preite, 2013). The highly variable and reversible nature of methylation patterns lead to rapid responses within a single generation, which under certain conditions are stable over the organism' lifespan and can even be transmitted to next generations (Kakunati et al. 1999, Vaughn et al. 2007, Johannes et al. 2009, Bossdorf et al. 2010, Boyko et al. 2010). Hence, it influences evolutionary rates and trajectories of natural populations, with consequences for the selection of asexual genotypes and, as a hereditary element, their spread within populations (Latzel \& Klimesova 2010, Massicotte \& Angers 2012).

Here, we studied methylation patterns among natural di- and tetraploid populations of the alpine herb Ranunculus kuepferi Greuter \& Burdet to investigate correlations to ploidy, reproduction mode and environmental influence along an altitude gradient in the European Alps between 1300 - $2800 \mathrm{~m}$. The distribution of cytotypes shows pronounced Geographical Parthenogenesis (Cosendai et al. 2011, 2013, Kirchheimer et al. 2016, 2018), with diploid populations confined to the southwestern Alps, while tetraploids colonize previously glaciated areas of higher elevations in the central and eastern Alps (Burnier et al. 2009, Cosendai \& Hörandl 2010). Tetraploid populations furthermore exhibited a pronounced niche shift towards colder temperatures (Kirchheimer et al. 2016, 2018; Schinkel et al. 2016). Diploid individuals reproduce sexually, with few exceptions of spontaneously generated apomictic seeds, tetraploid plants are facultative apomictic with varying proportions of sexual and asexual seed formation (Schinkel et al. 2016). Combination effects of niche shifts and mode of reproduction explain the colonization success of tetraploids in the Alps (Kirchheimer et al. 2018). Cosendai et al. $(2011,2013)$ had shown with AFLP and microsatellite studies that tetraploid populations of $R$. kuepferi are autopolyploids and show a similar level of genetic variation as their diploid 
progenitor populations. Therefore, we concentrated here on possible epigenetic variation, which might explain observed shifts in the reproduction mode and further adaptations of the tetraploid cytotype to their specific alpine environment (Schinkel et al. 2016, Kirchheimer et al. 2016).

Our study evaluates possible differences in epigenetic patterns between cytotypes and reproduction modes to gain better insights into correlations to expression of apomixis. To get a better understanding of the putative adaptive role of epigenetic mechanisms, we test for correlations of methylation patterns and important climatic variables. Altogether we try to understand the role of methylation patterns for explaining Geographical Parthenogenesis.

\section{Materials and Methods}

\section{a) Plant material}

Plants of Ranunculus kuepferi were collected from 81 localities throughout the whole distribution range in the Alps during field trips in 2013/14 and transferred to the Botanical Garden of the University of Goettingen as previously described by Schinkel et al. $(2016,2017)$. Voucher specimens have been deposited in the herbarium GOET. Leaf material of 1074 plants was collected directly in the field, to prevent influence of artificial stress (e.g. cut out, transfer) on the molecular analyses of methylation patterns, and subsequently dried and stored in silica gel. Ploidy levels of individuals were identified via flow cytometry with silica gel dried leaf material, while reproduction modes were determined using flow cytometric seed screening on five seeds per individual from 551 plants which provided enough seed material (Schinkel et al., 2016). We categorized individuals in four groups regarding their cytotype as well as their main reproduction mode, both determined by Schinkel et al. (2016): obligate sexual diploids (2xS), facultative apomictic diploids ( $2 \times \mathrm{M})$, obligate apomictic tetraploids ( $4 \times \mathrm{A})$ and facultative sexual tetraploids (4xM). "Obligate" means that a plant produced exclusively sexual (S) or apomictic seed (A), while "facultative" means that a plant produced both sexual and apomictic seeds (via various developmental pathways) in the same generation, with varying proportions; for simplicity of terminology, we denote the facultative mode as "mixed" (M) here. Among these we randomly chose 48 individuals for MSAP analysis of each group except for the 2xM group, as only six diploid individuals in total exhibited apomictic seed production 
(Schinkel et al. 2016). From the 150 chosen individuals 27 samples exhibited low quality signals in MSAP electropherograms even after several repetitions (see below) and therefore were excluded from further steps, resulting in 41 diploid, sexual (2xS), 6 diploid, mixed (2xM), 45 tetraploid, mixed ( $4 \times \mathrm{M})$ and 31 tetraploid, apomictic ( $4 \times \mathrm{A})$ scored samples. A list of the 123 individuals used for MSAP analyses with provenances and ploidy level/mode of reproduction is given in Appendix $\mathrm{A} 1$.

\section{b) Methylation-Sensitive Amplified Fragment-Length Polymorphisms}

DNA extraction was performed with QIAGEN DNeasy Plant Mini Kit (QIAGEN, Hilden, Germany) using a slightly modified protocol (see details in Appendix A2). Detection of epigenetic patterns was accomplished conducting methylation sensitive amplified fragment-length polymorphisms (MS-AFLP or MSAP). We followed the protocol of Paun et al. (2010) with some minor modifications (see below).

\section{Digestion and Ligation}

Digestion and ligation of DNA were performed subsequently as two independent reactions on each sample. We used two different restriction enzyme combinations: (i) Hpall (New England Biolabs, Ipswich, MA, USA) as frequent cutter and EcoRI (New England Biolabs, Ipswich, MA, USA) as rare cutter, (ii) Mspl (New England Biolabs, Ipswich, MA, USA) and EcoRI. Both

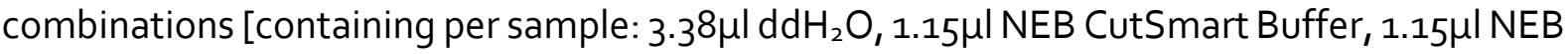
Mspl or Hpall respectively, $0.07 \mu \mathrm{l}$ NEB EcoRI-HF] were run in parallel under same conditions each with $5.75 \mu \mathrm{l}$ of the same DNA isolate. Hpall and Mspl are isoschizomeres with the same recognition sequence $\left(5^{\prime}-\mathrm{CCGG}-3^{\prime}\right)$ but a divergent sensitivity to cytosine methylation. Both enzymes cut a nonmethylated restriction site. Mspl cuts also if only the internal cytosine is either holo- or hemimethylated. Cleaving of Hpall is entirely blocked if either one or both cytosines are holomethylated, whereas hemimethylation on either or both cytosines only impairs restriction (McClelland et al. 1994, Roberts et al. 2007), which can be overcome with high fidelity enzymes, optimal digestion conditions and a prolonged incubation time. Digestion was performed at $37^{\circ} \mathrm{C}$ in a T100 Thermocycler (Bio-Rad Laboratories Inc., Hercules, CA, USA) for one hour.

Following digestion, adapters (Eurofins Scientific, Brussels, Belgium; see Appendix A2) were ligated adding $11.5 \mu \mathrm{l}$ reaction mix [containing per sample: $3.1 \mu \mathrm{l} \mathrm{ddH}_{2} \mathrm{O}, 2.3 \mu \mathrm{l}$ NEB T4 Ligase Buffer, $2.8 \mu \mathrm{l} 0.5 \mathrm{M} \mathrm{NaCl}, 1.15 \mu \mathrm{l}$ NEB Mspl or Hpall respectively, each $0.9 \mu \mathrm{l}$ EcoRI and 
Mspl/Hpall adapter mix, $0.35 \mu \mathrm{L}$ NEB T4 Ligase] and incubate for another hour at $37^{\circ} \mathrm{C}$ and subsequently over night at $16^{\circ} \mathrm{C}$. Products were screened for efficiency on a $1.5 \%$ agarose gel and diluted 10-fold prior to preselective amplification.

\section{Preselective and selective PCR}

First step reduction of fragments with preselective primers (Eurofins Scientific, Brussels, Belgium; see Appendix A2) was performed with a slightly modified master mix [containing per sample: $6.3 \mu \mathrm{lddH_{2 }} \mathrm{O}, 1.5 \mu \mathrm{l}$ NEB OneTaq Buffer, $1.5 \mu \mathrm{l} \mathrm{MgCl}_{2,} 0.3 \mu \mathrm{l}$ dNTP mix $25 \mu \mathrm{M}$ each, $0.7 \mu \mathrm{l}$ primer mix, $0.2 \mu \mathrm{l}$ NEB OneTaq] adding $4.5 \mu \mathrm{l}$ of diluted $\mathrm{R} / \mathrm{L}$ product on a T100 Thermocycler [1205 $94^{\circ} \mathrm{C} ; 20 \times 10594^{\circ} \mathrm{C}, 30562^{\circ} \mathrm{C}, 120572^{\circ} \mathrm{C} ; 30 \mathrm{~min} 60^{\circ} \mathrm{C} ; \infty 4^{\circ} \mathrm{C}$. Products were checked on a $1.5 \%$ agarose gel and diluted 10 -fold prior to selective amplification.

To obtain a manageable number of fragments we used three selective Hpall/EcoRI primer combinations, each with three selective nucleotides for Hpall and two for EcoRI, adapted from previous AFLP studies on R. kuepferi (Cosendai et al. 2013): Hpall+TCG/EcoRI+AC, Hpall+TGA/EcoRI+AC, Hpall+ATA/EcoRI+AC (Eurofins Scientific, Brussels, Belgium; see Appendix A2).

Three separate PCRs were conducted in parallel with same master mix [containing per sam-

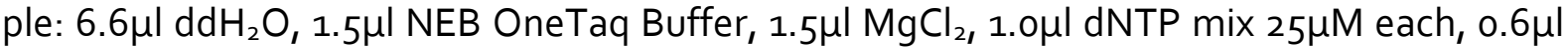
EcoRI primer, $0.6 \mu \mathrm{l}$ selective primer, $0.2 \mu \mathrm{l}$ NEB OneTaq] adding $3 \mu \mathrm{l}$ of preselective amplification product on T100 Thermocyclers $\left[120594^{\circ} \mathrm{C} ; 9 \times 10594^{\circ} \mathrm{C}, 30567^{\circ} \mathrm{C}-1^{\circ} \mathrm{C}\right.$ per cycle, $120 \mathrm{~S}$ $72^{\circ} \mathrm{C} ; 23 \times 10594^{\circ} \mathrm{C}, 30562^{\circ} \mathrm{C}, 120572^{\circ} \mathrm{C} ; 30 \mathrm{~min} 60^{\circ} \mathrm{C} ; \infty 4^{\circ} \mathrm{C}$. Products were kept cool at $4^{\circ} \mathrm{C}$ overnight and diluted 20 -fold prior to fragment analysis.

\section{Separation and Visualization of Fragments}

Fragment analyses were performed on an ABI Prism 3730 capillary sequencer (Applied Biosystems, Waltham, MA, USA) using GeneScan ROX 500 (Thermo Fisher Scientific, Waltham, MA, USA) as internal size standard. Quality was first checked visually with GeneMarker 2.7.4 software (SoftGenetics LLC., 16803 State College, PA, USA). As AFLP methods are prone to technical artifacts (e.g. false positive fragment peaks), we produced duplicates of every sample from restriction to selective $P C R$, to ensure overall reproducibility of resulting electropherograms (see also subsection "Statistical Analyses"). 


\section{Fragment scoring}

Analysis and interpretation of methylation patterns among samples and groups is based on present/absent profiles of fragments. Manual identification of electropherogram peaks is significantly biased by assessment quality of operating persons and further varies over time (Holland et al. 2008, Arrigo et al. 2009, Zhang and Hare 2012). Therefore, transformation of raw data to dominant binary matrices was conducted automatically using a scoring pipeline consisting of Peakscanner 2.0 software (Applied Biosystems, Waltham, MA, USA) for basic peak detection, the R package RawGeno 2.0-3 (Arrigo et al. 2009) for advanced peak or rather fragment identification as well as filtering of technical artifacts and non-reproducible fragments. To find optimal parameter combinations we ran a script incrementally increasing every parameter at a time and calculating relevant actuating factors on analysis quality (parameter settings in Appendix $\mathrm{A}_{3}$ ). Raw fragment recognition data from RawGeno were passed to MSAP_calc.r script that distinguishes possible methylation conditions comparing Hpall and Mspl profiles, as described in Schulz et al. (2013). Based on the differing sensitivity of the restriction enzyme isochizomers to methylation of their target sequence, four conditions can be distinguished: i) no methylation (both Hpall and Mspl cut), ii) holo- or hemimethylation of internal cytosine (MeCG respectively ${ }_{\mathrm{HMe}} \mathrm{CG}$, only Mspl cuts), iii) hemimethylation of external cytosine ( $\mathrm{HMe}_{\mathrm{C}} \mathrm{CHG}$, only Hpall cuts), iv) either holomethylation of both internal and external cytosine, or a mutation (both Hpall and Mspl do not cut). To transmit fragment patterns into a binary matrix for further analyses, we chose the Mixed Scoring 2 approach proposed by Schultz et al. (2013): condition i) was scored by the algorithm as " 0 " denoting „no methylation present", while conditions ii) \& iii) were scored as „1" and simultaneously „methylation present". Condition ii and Condition iii were separated during this scoring process. For this purpose, similar to a dummy coding, each locus was divided into three epiloci (nonmethylated, internally $\mathrm{MeCG}_{\mathrm{HMe}} \mathrm{CG}$-methylated, externally $\mathrm{HMe} \mathrm{CHG}$-methylated) and scored accordingly. Condition iv) was scored as „O" too, as it represents an ambiguous methylation or mutation status, which is not distinguishable.

\section{c) Statistical analysis}

All subsequent analyses were performed in R ( $R$ Development Core Team 2008) on basis of the largely automatically generated present/absent matrix containing the methylation status of every individual at 1088 epiloci (see above). Descriptive parameters were adopted from 
RawGeno and further explored in Rcmdr 2.4.4 (Fox 2005). Corresponding boxplots were created with ggplot2. Percentages were arcsine transformed to match a normal distribution of data. Pairwise ANOVA between groups were carried out in R using descriptive parameters of polymorphic loci distribution and abundance.

Principal exploration of groups according to methylation patterns was done performing a multidimensional scaling with the metric non-Euclidean Jaccard distance measure using the packages vegan 2.4-5 (Oksanen et al. 2017) and ggplot2 2.2.1 (Wickham 2009). Furthermore, a genetic distance-based group assignment was conducted using k-means with mclust 5.4.1 (Scrucca et al. 2017) in R to compare general methylation patterns in terms of their natural group appearance versus the minimized within-group diversity distribution/assignment based on parameterized Gaussian mixture models. The optimal model is selected according to Bayesian Information Criterion (BIC) by hierarchical clustering.

To compare the molecular epigenetic variances within and among our predefined groups (2xS, 2xM, 4xM, 4xA), as well as the cytotypes (diploid, tetraploid) and different reproduction modes (sexual, mixed $=2 \times M+4 \times M$, apomictic), we have calculated a total of 9 AMOVAs, based on the three different types of methylation (non-, internally-, externally methylated). In addition, the epigenetic phenotypic differentiation (DST) of loci was also determined by means of locus-by-locus AMOVA analyses. Both modes of AMOVA were executed in ARLEOUIN 3.5.22 (Excoffier et al. 2010), each for haplotypic data, a gamma of o.o and 50,000 permutations. To test separately for the factors ploidy $(2 x / 4 x)$ and mode of reproduction (sexual, mixed, apomictic), separate one- and two factorial ANOVAs were calculated and boxplots were produced.

Potential correlation between individual methylation patterns and spatial occurrences was tested with a stratified Mantel test in R with ecodist 2.0.1 (Goslee et al. 2007) using mismatch coefficients as suitable dissimilarity distances for dominant marker data (Kosman \& Leonard 2005). Geographic distances were calculated from population GPS centroid data. We furthermore calculated Moran's I (Moran 1947, 1948) to examine global spatial structuring over the entire sampling area, as well as Geary's C (Geary 1954) for more detailed local structure analysis. Moran's / values range between -1 and +1 , whereby positive values indicate spatial autocorrelation, a value near o random distribution, and negative values perfect dispersal. Geary's $C$ values are $>0$. Main environmental parameters for the distribution of the cytotypes (alti- 
tude, annual mean temperature, temperature of the coldest quarter, precipitation) were selected according to the study of Kirchheimer et al. (2016) and data were downloaded from WorldClim Database (Hijmans et al. 2005). The correlation of these parameters with observed methylation patterns were investigated with Samßada 0.5.3 (Stucki et al. 2017). Samßada uses logistic regressions to model the probability of observing a particular genotype of a polymorphic marker given the environmental conditions at the sampling locations based on local Moran's I values (Joostet al. 2007). Our chosen multivariate approach with four environmental predictor variables is similar to a forward stepwise regression (Dobson \& Barnett 2008). Model selection was based on Wald and $G$ test statistics. Associations between observed candidate loci and environmental parameters were further tested with generalized linear mixed model analyses (GLMM, see Bolker et al. 2008) in R with Ime4 (Douglas et al. 2015). 


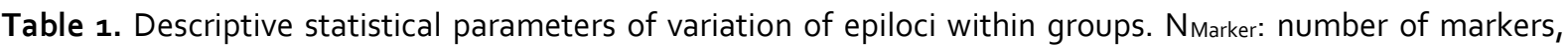
(Polym: total number of polymorphic markers, [\%]Poly: percentage of of polymorphic markers, Private: number of private polymorphic markers, [\%] Priv: percentage of of private polymorphic markers), $\mathrm{H}^{\prime}$ Shannon: Mean Shannon Diversity Index.

\begin{tabular}{|c|c|c|c|c|c|c|}
\hline & \multicolumn{6}{|c|}{ nonmethylated } \\
\hline & \multicolumn{5}{|c|}{ NMarker } & \multirow{2}{*}{$\begin{array}{l}\text { H'Shannor } \\
\text { Total }\end{array}$} \\
\hline & Total & Polym & {$[\%]_{\text {Poly }}$} & Private & {$[\%]_{\text {Priv }}$} & \\
\hline $2 \times S$ & 268 & 162 & 60.5 & 40 & $24 \cdot 7$ & 0.285 \\
\hline $2 \times M$ & 268 & 85 & 31.7 & 6 & 7.1 & 0.248 \\
\hline $4 \times M$ & 268 & 190 & 70.9 & 64 & 33.7 & 0.326 \\
\hline \multirow[t]{4}{*}{$4 \times A$} & 268 & 48 & $17 \cdot 9$ & 2 & 4.2 & 0.109 \\
\hline & \multicolumn{6}{|c|}{ externally methylated } \\
\hline & \multicolumn{5}{|l|}{ NMarker } & H'Shannon \\
\hline & Total & Polym & {$[\%]_{\text {Poly }}$} & Private & {$[\%]_{\text {Priv }}$} & Total \\
\hline $2 \times S$ & 378 & 260 & 48.8 & 46 & 17.7 & 0.323 \\
\hline $2 \times M$ & 378 & 139 & 31.4 & 4 & 2.9 & 0.358 \\
\hline $4 \times M$ & 378 & 197 & 44.6 & 20 & 10.2 & 0.311 \\
\hline \multirow[t]{4}{*}{$4 \times A$} & 378 & 309 & 69.9 & 69 & 22.3 & 0.088 \\
\hline & \multicolumn{6}{|c|}{ internally methylated } \\
\hline & \multicolumn{5}{|c|}{ NMarker } & H'Shannon \\
\hline & Total & Polym & {$[\%]_{\text {Poly }}$} & Private & {$[\%]_{\text {Priv }}$} & Total \\
\hline $2 \times S$ & 442 & 259 & 68.5 & 53 & 20.5 & 0.235 \\
\hline $2 \times M$ & 442 & 176 & 46.6 & 14 & 7.9 & 0.228 \\
\hline $4 \times M$ & 442 & 242 & 64.1 & 56 & 23.1 & 0.173 \\
\hline $4 \times A$ & 442 & 62 & 16.4 & 6 & $9 \cdot 7$ & 0.325 \\
\hline
\end{tabular}

Table 2. Results of pairwise comparisons of groups with ANOVA for the different types of epiloci. Pairs with either equal ploidy or equal mode of reproduction marked in bold. EST: Estimate, SE: Standard Error, $t$ : $t$-value, $p$ : p-value.

\begin{tabular}{|c|c|c|c|c|c|c|}
\hline & Pair & & EST & SE & $\mathrm{t}$ & $p$ \\
\hline \multirow[t]{6}{*}{ nonmethylated } & $2 \times S$ & $-2 \times M$ & 10.365 & 2.967 & 3.494 & 0.004 \\
\hline & $2 \times S$ & $-\quad 4 x M$ & -6.311 & 1.473 & -4.284 & $<0.001$ \\
\hline & $2 \times S$ & $-\quad 4 \times A$ & 22.439 & 1.615 & 13.891 & $<0.001$ \\
\hline & $2 \times M$ & $-\quad 4 \times M$ & -16.676 & 2.954 & -5.646 & $<0.001$ \\
\hline & $2 \times M$ & $-\quad 4 \times A$ & 12.074 & 3.027 & 3.989 & $<0.001$ \\
\hline & $4 \times M$ & $-\quad 4 \times A$ & 28.75 & 1.591 & 18.065 & $<0.001$ \\
\hline
\end{tabular}




\begin{tabular}{|c|c|c|c|c|c|c|}
\hline \multirow[t]{6}{*}{ externally methylated } & $2 \times S$ & $-2 \times M$ & -4.935 & 3.736 & -3.736 & 0.536 \\
\hline & $2 \times S$ & $-\quad 4 \times M$ & $-3 \cdot 423$ & 1.855 & -1.855 & 0.245 \\
\hline & $2 \times S$ & $-\quad 4 X A$ & -50.212 & 2.035 & -2.035 & $<0.001$ \\
\hline & $2 \times M$ & $-\quad 4 \times M$ & 1.513 & 3.72 & -3.72 & 0.976 \\
\hline & $2 \times M$ & $-\quad 4 \times A$ & -45.277 & 3.812 & -3.812 & $<0.001$ \\
\hline & $4 \times M$ & $-\quad 4 \times A$ & -46.79 & 2.004 & -2.004 & $<0.001$ \\
\hline \multirow[t]{6}{*}{ internally methylated } & $2 \times S$ & $-\quad 2 \times M$ & -5.43 & $3 \cdot 508$ & -1.548 & 0.397 \\
\hline & $2 \times S$ & $-\quad 4 \times M$ & 9.734 & 1.742 & $5 \cdot 588$ & $<0.001$ \\
\hline & $2 \times S$ & $-\quad 4 \times A$ & 27.772 & 1.91 & $14 \cdot 541$ & $<0.001$ \\
\hline & $2 \times M$ & $-\quad 4 \times M$ & 15.164 & 3.492 & 4.342 & 0.002 \\
\hline & $2 \times M$ & $-\quad 4 \times A$ & 33.202 & 3.579 & 9.277 & $<0.001$ \\
\hline & $4 \times M$ & $-\quad 4 \times A$ & 18.038 & 1.882 & $9 \cdot 586$ & $<0.001$ \\
\hline
\end{tabular}

\section{Results}

\section{a) Epigenetic variation within and among groups}

Methylation-sensitive AFLP genotyping revealed 681, 400, 629 and 419 polymorphic markers among 1088 scorable fragments in total in the four groups $2 \times S, 2 \times M, 4 \times M$, and $4 \times A$, respectively. Number of private markers was lowest among 2xM (24) and equally high for 2xS and 4xM (140). Epigenetic diversity measured with mean Shannon diversity indices was lowest within the asexually reproducing $4 \times \mathrm{A}$ group $\left(H^{\prime}=.189\right)$ and ranged from $H^{\prime}=.259$ to $H^{\prime}=.278$ in the other groups. More pronounced differences were found in the marker status comparison between non-, external- and internally methylated epiloci, where $4 \times \mathrm{A}$ possessed significantly less non-methylated $(17.91 \%, p<.001)$ and externally methylated $(16.40 \%, p<.001)$, but significantly increased internally methylated $(69.91 \%, p<.001)$ markers compared to the three other groups (Table 1). Pairwise comparisons of groups with ANOVAs supported these findings (Table 2 ) and revealed highly significant differences between $2 \times S$ and $4 \times \mathrm{A}(p<.001)$ among all epiloci subsets.

Multidimensional scaling diagrams show noticeable differentiation between the four predefined groups (Figure 1). Clear separation is found according to respective cytotypes, as well as within the tetraploids regarding their main reproduction modes, with $4 \times \mathrm{M}$ and $4 \times \mathrm{A}$ forming each distinct cluster. Among diploids the 2xS and 2xM samples are basically admixed and cluster together, although one sample of the $2 x \mathrm{M}$ group tends distinctly to the $4 \times \mathrm{A}$ cluster. 
The general picture remains largely the same considering the different epiloci subsets. Differences occur in particular at the NMDS of internally methylated markers (Figure 1D), with a broader scattering in the $4 \times A$ group.
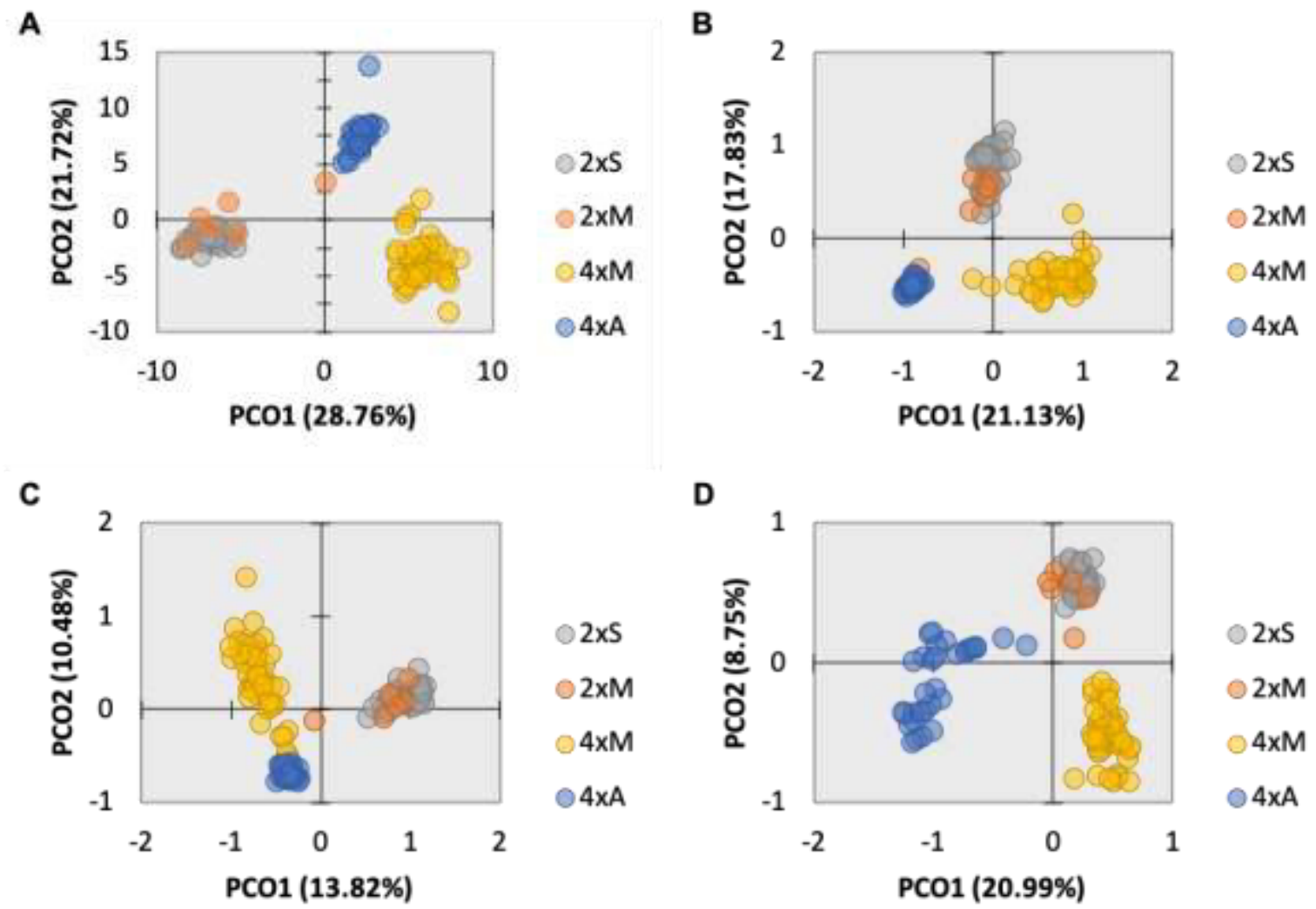

Figure 1. Non-Metrical Dimensional Scaling (NMDS) plots of epiloci. A: all epiloci combined, B: nonmethylated epiloci, C: externally methylated epiloci, D: internally methylated epiloci.

Based on the total epiloci, it appears that K-means clustering reproduces the predefined groups fairly well (Figure 2). Especially with respect to the tetraploid groups $4 \times \mathrm{XM}$ and $4 \times \mathrm{A}$, all samples of each are classified into a respective K-means cluster. However, diploid individuals are grouped together into one cluster. Further, one sample of the $2 x \mathrm{M}$ group is also assigned to the cluster in which all $4 \times \mathrm{A}$ individuals are located. This is also evident when looking at the nonmethylated epiloci subset, but here one sample of the $4 \times \mathrm{M}$ group is assigned to the $4 \mathrm{XA}$ cluster. Both other subsets show a different distribution. Among externally methylated epiloci all tetraploid samples form a cluster, while one sample of $2 \times M$ corresponds to the $4 \times A$ cluster. Among methylated epiloci however, diploids form a cluster together with the $4 \times \mathrm{M}$ group. Here an individual from $4 \times \mathrm{A}$ is assigned to the $4 \times \mathrm{M}$ cluster. 

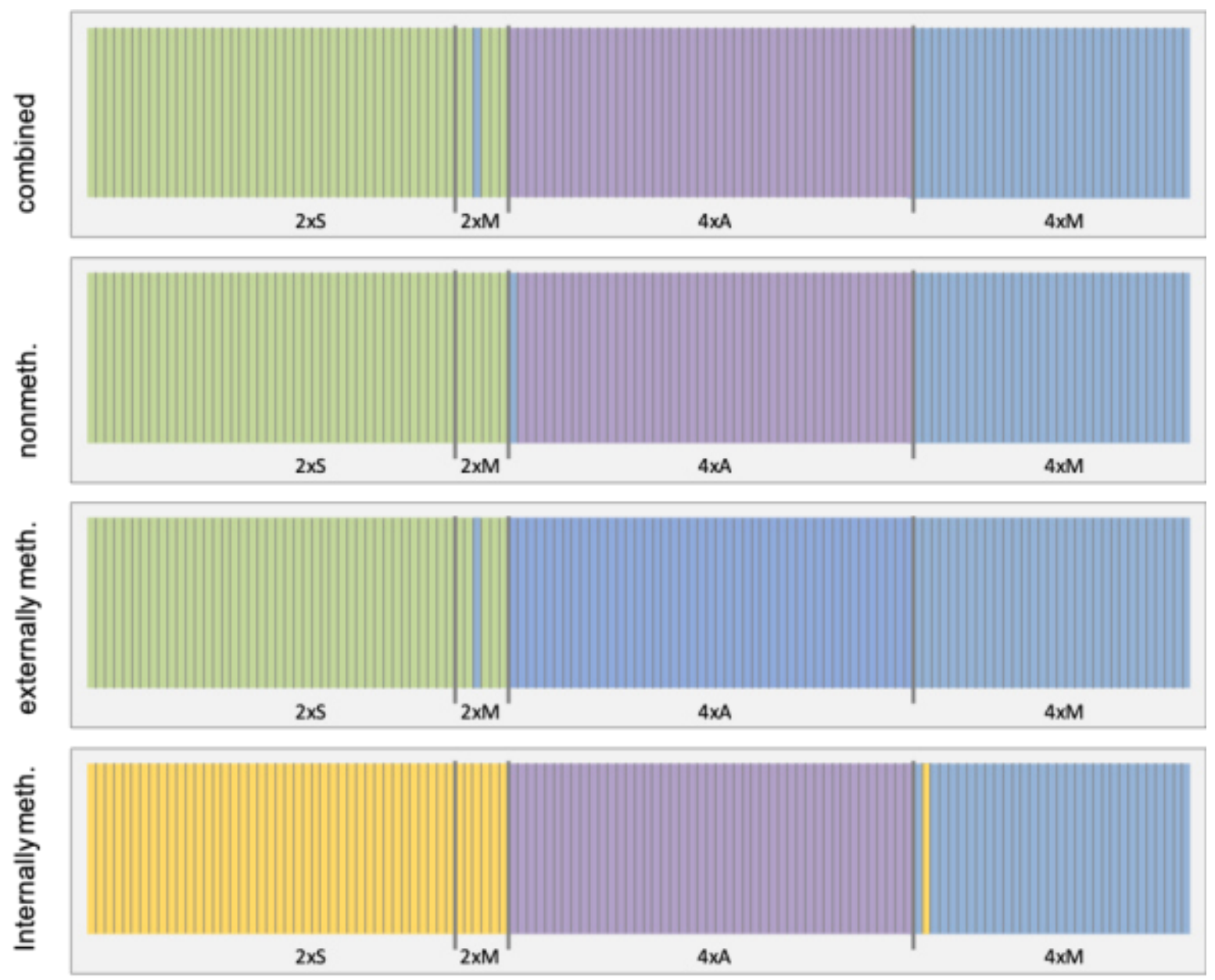

Figure 2. Pseudo barplots of K-means clustering group assignment of epiloci. Individual classification is indicated by different colours.

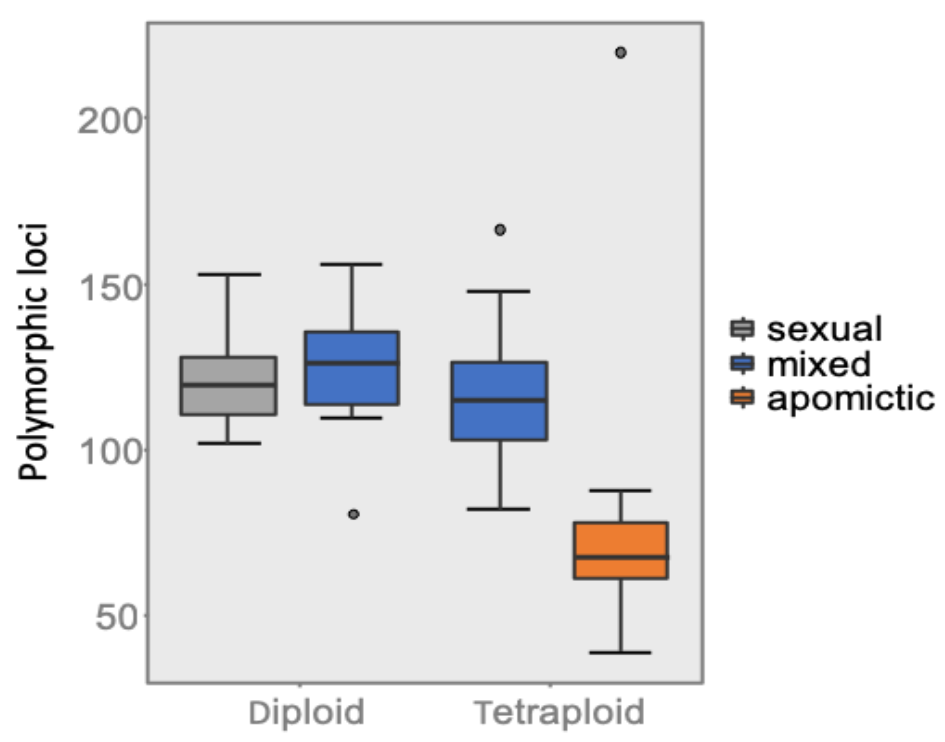

Boxplots summarizing all epiloci indicate a lower amount of polymorphic epiloci for the $4 \times A$ group compared to the other groups, which exhibit similar variation (Figure 3). This is mostly due to a lower amount of non-methylated and externally methylated epiloci (not shown).

Figure 3. Boxplot of polymorphic epiloci counts by means of ploidy and reproduction mode. 
Table 3. Results of molecular variance analyses (AMOVA) of epiloci. df: Degrees of freedom, Fsc: Differences among collec-tions within groups, $F_{\mathrm{ST}}$ : differences among all collections, $p$ : $\mathrm{p}$-value.

\begin{tabular}{|c|c|c|c|c|c|c|}
\hline nonmethylated & & & & & & \\
\hline Source of variation & $\mathrm{df}$ & Variance components & $\mathrm{p}$ & $\%$ variation & F stat & stics \\
\hline Among groups & 3 & 11.510 & $<0.001$ & 42.95 & Fsc: & 0.169 \\
\hline Within groups & 119 & 15.287 & $<0.001$ & 57.05 & FST: $_{\text {ST }}$ & 0.526 \\
\hline Among cytotypes & 1 & $7 \cdot 375$ & $<0.001$ & 27.11 & Fsc: & 0.359 \\
\hline Within cytotypes & 121 & 19.828 & $<0.001$ & 72.89 & $\mathrm{FST}_{\mathrm{ST}}$ & 0.533 \\
\hline Among reproduction modes & 2 & 7.129 & $<0.001$ & 26.14 & Fsc: & 0.369 \\
\hline Within reproduction modes & 120 & 20.149 & $<0.001$ & 73.86 & Fst: & 0.534 \\
\hline internally methylated & & & & & & \\
\hline Source of variation & $\mathrm{df}$ & Variance components & $\mathrm{p}$ & $\%$ variation & F stat & stics \\
\hline Among groups & 3 & 10.900 & $<0.001$ & 32.98 & $F_{s c:}$ & 0.177 \\
\hline Within groups & 119 & 22.151 & $<0.001$ & 67.02 & Fst: & 0.448 \\
\hline Among cytotypes & 1 & 11.501 & $<0.001$ & 32.15 & Fsc: & 0.249 \\
\hline Within cytotypes & 121 & 24.275 & $<0.001$ & 67.85 & FST: & 0.491 \\
\hline Among reproduction modes & 2 & 10.515 & $<0.001$ & 29.57 & Fsc: & 0.272 \\
\hline Within reproduction modes & 120 & 25.047 & $<0.001$ & 70.43 & $\mathrm{FST}_{\mathrm{ST}}$ & 0.487 \\
\hline externally methylated & & & & & & \\
\hline Source of variation & $\mathrm{df}$ & Variance components & $\mathrm{p}$ & $\%$ variation & $F$ stat & stics \\
\hline Among groups & 3 & 8.526 & $<0.001$ & 27.68 & Fsc: & 0.124 \\
\hline Within groups & 119 & 22.278 & $<0.001$ & 72.32 & FST: & 0.366 \\
\hline Among cytotypes & 1 & 5.011 & $<0.001$ & 16.23 & Fsc: & 0.245 \\
\hline Within cytotypes & 121 & 25.859 & $<0.001$ & 83.77 & Fst: & 0.367 \\
\hline Among reproduction modes & 2 & 4.531 & $<0.001$ & 14.74 & Fsc: & 0.255 \\
\hline Within reproduction modes & 120 & 26.216 & $<0.001$ & 85.26 & FST: & 0.365 \\
\hline
\end{tabular}


Table 4. Results of two level hierarchical AMOVA of epiloci with cytotype as first and reproduction mode as second level. SSD: Squared standard deviation, MSD: Mean standard deviation, df: Degrees of freedom, Sigma²: representation of variance measures, $p$ : $\mathrm{p}$-value.

\begin{tabular}{lllllll}
\hline & SSD & MSD & df & \multicolumn{2}{l}{ Variance coefficients Sigma $^{2}$} & $p$ \\
\hline Cytotype & 7.437 & 7.437 & 1 & 45.46 & 0.059 & $<0.001$ \\
Reproduction mode & 6.036 & 6.036 & 1 & 34.92 & 0.127 & $<0.001$ \\
Error & 31.135 & 0.259 & 120 & 46.37 & 0.259 & \\
Total & 44.609 & 0.366 & 122 & & & \\
\hline
\end{tabular}

\section{b) Correlations of MSAPs to ploidy level versus reproduction mode}

Pairwise comparisons of groups, where either ploidy (2xS - 2xM and $4 \times \mathrm{M}-4 \times \mathrm{A}$ ) or mode of reproduction ( $2 \times M-4 \times M)$ could be kept equal, indicate also significant differences $(p<0.005)$ in types of epiloci (Table 2): nonmethylated and internally methylated loci differ significantly between cytotypes with the same mode of reproduction (2xM $-4 \times M)$, nonmethylated and externally methylated loci differ between sexual and mixed reproduction in diploids (2xS $2 \times M)$, and all three epiloci differ between mixed and apomictic reproduction in tetraploids $(4 \times M-4 \times A)$.

AMOVA indicate epigenetic differentiation to be higher within groups than among them, also regarding the epigenetic differentiation with respect to cytotype as well as reproduction mode as grouping factors (Table 3). If the differences in cytotype and reproduction are considered individually with respect to the different epiloci, the variance is also greatest in externally methylated markers. Separate hierarchical AMOVA revealed a significant influence of both cytotype $(p<.001)$ and reproduction mode $(p<.001)$ on the total epigenetic variances found (Table 4). $F_{\text {st }}$ values for the non- and externally methylated epiloci commute around 0.5 (nonmethylated $0.526-0.534$; externally methylated $0.448-0.491$ ), implying an equivalent origin of the epigenetic variance between panmixis and population structural influences. Internally methylated epiloci exhibit distinctly lower $F_{s t}$ values $(0.365-0.367)$. Regarding locus-by-locus AMOVA, the overall percentages of significantly differentiated epiloci ranged from $4.17 \%(2 \times M)$ to $32.99 \%(4 \times M)$. Significant differences are found mainly between $2 \times S$ and $2 \times M, 4 \times A$, as well as $4 \times M$ and $2 \times M, 4 \times A$.

One-factorial ANOVA likewise indicate significant deviations between cytotypes $[F(1,364)=$ 14.29; $p<.001]$ and reproduction mode $[F(2,363)=25.31 ; p<.001]$ but not methylation status $[F(2,363)=1.69 ; p<.187]$. Two-factorial ANOVA found significant differences in methylation by reproduction mode $[F(2,362)=17.59 ; p<.001]$, but not ploidy $[F(2,362)=0.25 ; p<.618]$. 
Table 5. Moran's / values of epiloci for between group in correlation to spatial structure (2xS: Diploid, sexual; 2xM: Diploid, mixed; 4xM: Tetraploid, mixed; 4xA: Tetraploid, apomictic) and methylation status comparisons. lobs $=$ Observed Moran's I, lest = Estimated Moran's $I$.

\begin{tabular}{|c|c|c|c|c|c|c|c|c|c|c|c|c|}
\hline & $2 \times S$ & & & $2 \times M$ & & & $4 \times M$ & & & $4 \times A$ & & \\
\hline & lobs & lest & $p$ & lobs & lest & $\mathrm{p}$ & lobs & lest & $\mathrm{p}$ & lobs & lest & $p$ \\
\hline nonmethylated & 0.201 & -0.008 & 0.462 & 0.028 & -0.004 & 0.468 & 0.042 & -0.008 & 0.439 & 0.058 & -0.009 & 0.441 \\
\hline $\begin{array}{l}\text { externally } \\
\text { methylated }\end{array}$ & 0.191 & -0.008 & 0.455 & 0.047 & -0.009 & 0.475 & 0.062 & -0.007 & 0.446 & 0.044 & -0.008 & 0.444 \\
\hline $\begin{array}{l}\text { internally } \\
\text { methylated }\end{array}$ & 0.136 & -0.007 & 0.472 & 0.027 & -0.007 & 0.482 & 0.013 & -0.008 & 0.451 & 0.062 & -0.008 & 0.444 \\
\hline
\end{tabular}

\section{c) Geographical and spatial effects}

Test of correlation of epigenetic variances to geographical occurrence of individual plants with a stratified Mantel test revealed no significant structuring, neither among $(r=.155, p=$ .864 ) nor within cytotypes (diploids: $r=.136, p=.721$; tetraploids: $r=.167, p=.927$ ). This remained unchanged, considering the results of the Mantel tests separately for the subsets of non-, externally- and internally methylated epiloci for diploids as well as tetraploids (all $p$ valves $>0.05$ ). To exclude any falsification of the results by spatial autocorrelation, the analysis of Moran's / showed low observed values around zero among all groups and methylation status (Table 5), as expected by estimated values of simulations and therefore no significant deviations. This indicates that no spatial autocorrelation exists at the global level of our study area. In contrast, we observed a considerable amount of high values (>1) for Geary's $C$ among 18 individuals of $2 \times S, 14$ of $4 \times M$ and 4 of $4 \times A$. Values range from $C=1.178$ to $C=1.901$ in $2 \times S$, $C=1.033$ to $C=4.401$ in $4 \times M$ and $C=1.262$ to $C=1.908$ in $4 \times A$, exhibiting strong negative spatial autocorrelation.

Table 6. Moran's I values in correlation to observed environmental parameters. AMT: Annual mean temperature (BIO1, WorldClim), AP: Annual precipitation (BIO12, WorldClim).

\begin{tabular}{llll}
\hline & Altitude & AMT & AP \\
\hline Diploids & 0.845 & 0.789 & 0.751 \\
Tetraploids & 0.700 & 0.682 & 0.791 \\
\hline
\end{tabular}




\section{d) Environmental influences}

We observed that global values of Moran's / correlate with environmental factors. For all parameters (altitude, mean annual temperature (see $\mathrm{BIO}_{1}$ in WorldClim), annual precipitation (see $\mathrm{BIO}_{12}$ in WorldClim) a high value near 1 is shown, both for diploids and tetraploids (Table $6)$.

On local scale, correlations of high values for Geary's C among all groups are evenly distributed to altitude, mean annual temperature and annual precipitation (Appendix $\mathrm{A}_{4}$ ). We found some clustering of high $C$ values in either one of the environmental variables in connection with geographically closely spaced populations, but no significant tendency for one or another group regarding a certain correlation prevalence to one of the three parameters.

General linear mixed models revealed significant correlations between epigenetic variation and altitude (Figure 4) among 22 epiloci (non: 6; externally: 15; internally: 1), as well as mean annual Temperature (not shown) among 36 epiloci (non: 9; externally: 23; internally: 4). It is noticeable that three of each non- and internally-, as well as 8 externally methylated epiloci are solely correlated to mean annual temperature (Appendix A5). We could not find candidate epiloci with significant correlations to annual precipitation.

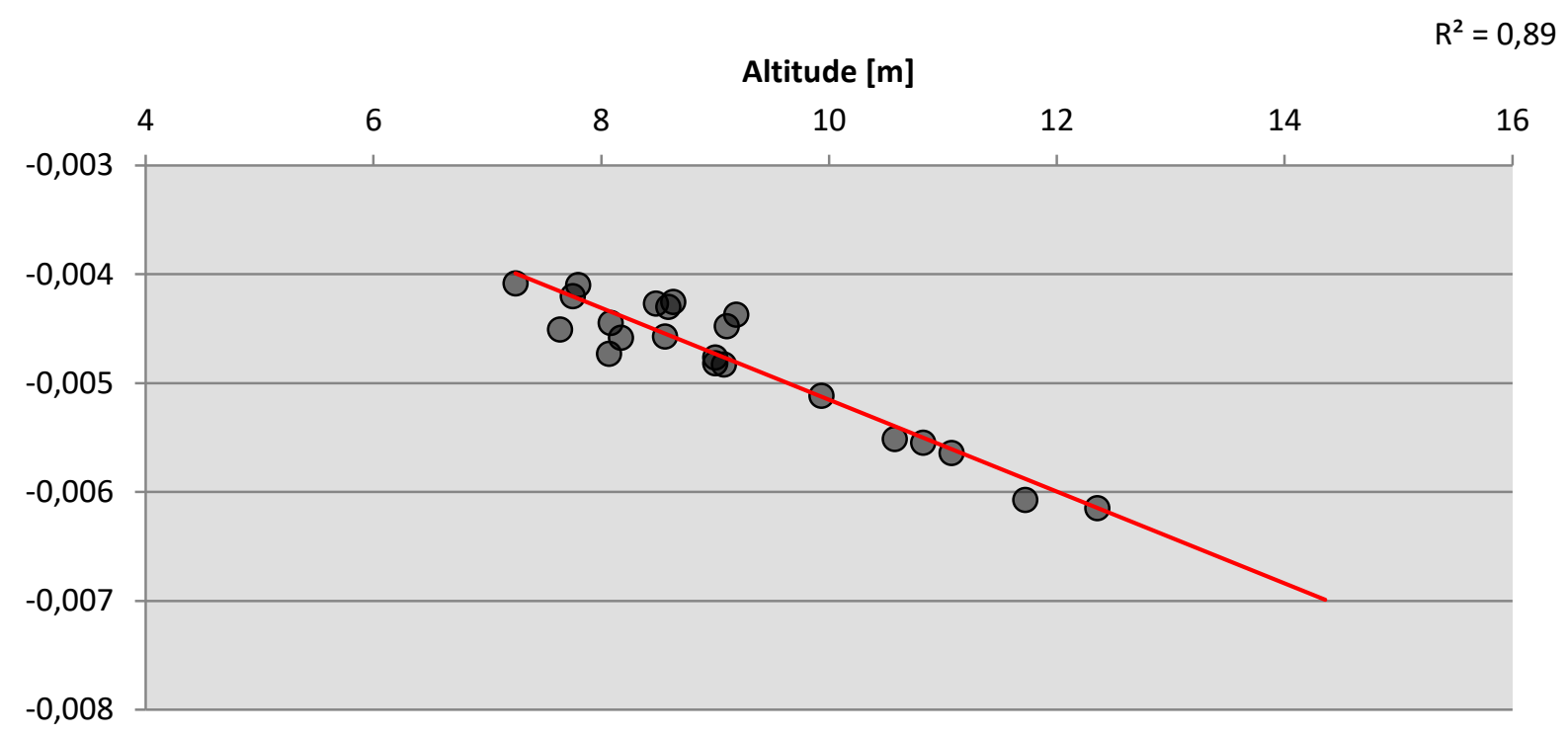

Figure 4. Scatterplot of Generalized Linear Model regression of epiloci with altitude as fixed factor. 


\section{Discussion}

Our study explored epigenetic patterns in di- and tetraploid populations of $R$. kuepferi, along with observed differences in the respective reproduction mode. We hypothesized that polyploidy as well as apomictic seed production result in distinct patterns of epigenetic variation: (i) epigenetic differentiation between di- and tetraploids, (il) epigenetic differentiation between obligate and facultative reproduction modes. We further tested for correlations of methylation patterns to spatial distribution and key climatic variables to understand their role for the observed niche shifts.

We found significant epigenetic differentiation between cytotypes as well as between apomictic and mixed reproducing tetraploids. Among diploids, a differentiation regarding their reproduction mode cannot be determined with certainty due to limited sample size. Among tetraploids, a striking difference was found between mixed and obligate apomictic reproduction.

A general correlation of the observed methylation patterns with environmental parameters was found for both altitude and annual mean temperature. This does reflect the regional distribution and climatic niches of the cytotypes. A significant differentiation between di- and tetraploids in a fraction of epiloci could be likewise traced to altitude and annual mean temperature. These candidate loci are particularly interesting as potential contributors to phenotypic plasticity and adaptation, which may have played a crucial role in the niche shift of apomictic tetraploid $R$. kuepferi.

\section{a) Epigenetic patterns}

Our NMDS plots show a clear distinction of samples regarding our predefined groups (2xS, $2 \times M, 4 \times M, 4 \times A)$. Of particular interest is the noticeable separation among tetraploids between obligatory apomictic and mixed ones. Obligate apomicts tend to cluster more densely, which is particularly evident in nonmethylated epiloci. This is probably related to the largely clonal reproduction caused by asexuality. Surprisingly, there is a greater variability among apomictic tetraploids regarding the internally methylated epiloci. Samples of diploids with mixed reproduction cluster within the obligate sexual diploids, even in nonmethylated epiloci, probably due to the very small sample size in the mixed reproducing diploids. An outlier is one sample of a diploid individual, which in all analyses was assigned to the apomictic tetraploid, also clustering close to them in NMDS plots. This individual had a particularly high 
apomictic reproductive rate (90\%) in our previous Flow Cytometry Seed Screening (FCSS) study (see Schinkel et al. 2016). Also, this finding supports a hypothesis of an influence of epigenetic patterns on mode of reproduction

Results of our k-means clustering analysis provide further indications of separate correlations of ploidy on internally methylated epiloci and reproduction mode on externally methylated epiloci. Among the internally methylated epiloci a distinct clustering according to ploidy level was found $(k=2)$, grouping each di- and tetraploid samples together independent of their respective reproduction mode. In contrast, among externally methylated epiloci an assignment of both diploid groups together with mixed reproducing tetraploids against apomictic ones rather represents partitioning due to reproduction.

In this context, the relative contribution of $\mathrm{CG}$ or $\mathrm{CHG}$ and $\mathrm{CHH}$ sequence motifs to the global genomic cytosine content may play a crucial role. It has previously been shown that the ratios between methylation on CG to CHG contexts vary across plant species and could therefore be species-specific (Gruenbaum et al. 1981, Belanger and Hepburn 1990, Kovarik et al. 1997, Feng et al. 2010, Vanyushin \& Ashapkin 2011, Zhong et al. 2013, Alonso et al. 2016, Gouil \& Baulcombe 2016, Takuno et al. 2016). Regarding the interpretation of MSAP results in $R$. kuepferi, it is unknown to which proportion the detected internally methylated epiloci are hemi- or even holomethylated, but the CG epiloci differ between the two cytotypes in all comparisons (Table 2). Internal holomethylation (CG context) could mean that these epiloci have a more heritable yet conserved methylation pattern (Paszkowski and Grossniklaus 2011, Takuno and Gaut 2013), which the polyploid cytotype has stabilized after its origin. External methylations, which are less stable and only partly heritable (Becker et al. 2011), appear to relate in $R$. kuepferi more to mode of reproduction, as they do not differ between the cytotypes ( $2 \times M$ and $4 \times M$ ), but differentiate the mode of reproduction ( $4 \times M$ and the $4 \times A$ ). Mode of reproduction is indeed variable under different environmental conditions and shows phenotypic plasticity in the same individuals over flowering seasons (Klatt et al. 2018).

In addition, changes in methylation status at CG or CHG depend on two distinct families of methyltransferases (Teixera and Colot 2009, Finnegan 2010, Law and Jacobsen 2010, Zemach et al. 2013, Stroud et al. 2014), which in turn means that both pathways can be regulated independently. Interestingly enough, some evidence suggests that these specific relationships between global methylation and the two nucleotide sequence contexts may have 
an important impact at population level and even in respect to polyploidization or ploidy alteration (Zhang et al. 2015, Gao et al. 2016, Baidouri et al. 2018).

Global AMOVAs revealed a higher variation within groups than among them. This trend is more pronounced comparing cytotypes and reproduction modes. Low FST values around 0.15 (Frankham et al. 2010) would indicate variation within groups, but not a significant differentiation between cytotypes or reproduction modes. Variation and differentiation of epigenetic patterns within groups are probably influenced not only by individual genotypes, but also by local microniche conditions of sampling sites and age of the individual plant when leaves were collected in the wild.

Pairwise comparisons largely support our previous statements but show a more detailed picture on the respective differences of cytotypes versus mode of reproduction. Our ANOVAs exhibit that differentiation due to ploidy is evident among methylated loci, but not unmethylated epiloci. These findings are in accordance with Cosendai et al. (2013), which found the genetic differentiation measured with AFLP markers and Fsts to be comparably high between di- and tetraploid accessions of $R$. kuepferi. Nevertheless, besides numerous genetic and physiological influences, polyploidy also seems to have epigenetic consequences and in particular affects methylation (Mittelsten et al. 2003, Li et al. 2011, Madlung and Wendel 2013). Epigenetic mechanisms such as DNA methylation can be influenced so that the gene expression of both allo- and autopolyploids is altered (e.g. gene silencing) and it can lead to a genome-wide transcriptional reprogramming (Bird 2002, Yan et al. 2010, Law and Jacobsen 2010, Baubec et al. 2010, Song and Chen 2016). Therefore, we would expect a clear separation of the methylation patterns between di- and tetraploid groups or samples, as before. Contradictory, diploids with mixed reproduction seem to be more similar to tetraploids with likewise mixed reproduction. This is particularly interesting because in a previous study we hypothesized that tetraploid $R$. kuepferi were formed by a triploid bridge from diploid individuals that occasionally produce apomictic seeds (Schinkel et al. 2017). These diploids may have had an epigenetic background for this transition.

Considering only the reproduction mode independent of ploidy, ANOVA results show no significant difference of methylation patterns between sexual and mixed reproduction. In fact, both groups of diploids seem to be rather similar, which is admittedly due to a small sample size in the mixed diploids, as previously mentioned. But in contrast, types of reproduction among tetraploids significantly differentiate the individuals of both groups $(4 \times M, 4 \times A)$ in 
ANOVA and pairwise comparisons. This is especially pronounced in the subset of externally methylated epiloci among which p-values are exceedingly high, may indicating higher rates of possible de novo methylation (Gent et al. 2013).

Mixed reproduction in $R$. kuepferi represents partly high rates of facultative sexuality (Schinkel et al. 2016), which in turn requires a certain degree of genetic coordination to grant undisturbed operating meiosis, embryo sac development and fertilization (Carman 1997). In general, gene regulations are predominantly implemented by epigenetic mechanisms such as methylation and seem to be highly pronounced in plants (e.g. Pikaard \& Scheid 2014). High rates of sexuality among mixed reproducing individuals of either di- or tetraploids may demand similar methylation patterns, as these may represent similar genetic regulation networks necessary for preservation of sexual pathways. Grimanelli (2012) proposed that apomixis is likewise under epigenetic control. Klatt et al. (2018) observed increasing proportions of apomictic seed formation in diploid R. kuepferi under experimental cold conditions, whereas there was no significant effect on seed proportions in tetraploid apomicts. Deviations from sexual reproduction can affect the inheritance of methylation patterns, as epigenetic re-setting may take place during the meiotic phases in sexual reproduction which occurs during facultative apomixis (Santos et al. 2002, Slotkin et al. 2009). However, apomictic reproduction is more likely to show a spatial and temporal deregulation of sexual developmental processes (Koltunow and Grossniklaus 2003). There are accumulating indications that a demethylation may be responsible for the interrupt of epigenetic control among regulating genes and networks with concomitant overexpression (Laspina et al. 2008, Polegri et al. 2010, Felitti et al. 2011, Podio et al. 2014, Rodrigo et al. 2017, Bocchini et al. 2018). This, in turn, could lead to disruption of the precisely controlled and orchestrated gene expression as prerequisite for a functioning meiosis. Obligate apomixis is then something like a way out of sterility (see Hörandl 2009, Hojsgaard et al. 2014) caused by epigenetic deregulation of gene expression. As expected, we found the amount of externally methylated fragments among apomictic tetraploids at a low level of $16.4 \%$, much less than among the other groups. Demethylation is actually mainly known as a reaction to stress factors e.g. temperature, drought, herbivory (e. g. Boyko \& Kovalchuk 2008, Chinnusamy \& Zhu 2009, Verhoeven et al. 2010, Dowen et al. 2012 Feil \& Fraga 2012, Shan et al. 2013, Kinoshita \& Seki 2014).

Strikingly, we found the described different methylation patterns related to mode of reproduction in vegetative tissues (basal leaves; flowers were not sampled as they were used to 
determine mode of reproduction). This observation supports a hypothesis by Hörandl \& Hadacek (2013) and Klatt et al. (2016) that the physiological stress response ability of the whole plant has an influence on mode of reproduction. Shah et al. (2016) indeed found in seedlings of obligate apomictic Boechera a global deregulation of expression of both meiotic and stress response genes compared to sexual ones, whereby gene expression changes could be induced by global DNA demethylation. The authors concluded that a global stress reprogramming of the plant might occur prior to apomixis initiation. In facultative apomictic Eragrostis curvula, proportions of sexuality increased after stress treatments, and this was correlated to changes in methylation patterns (Rodrigo et al. 2017). The obligate $4 \times$ apomictic plants of $R$. kuepferi showed a much lower amount of epiloci than their diploid progenitors in leaves. Hence, the 4XA group might represent lineages that have experienced cold conditions and demethylations, consequently establishing apomictic reproduction. Apomixis, in turn, helps to maintain these methylation profiles over generations as meiotic resetting is bypassed.

\section{b) Geographical patterns and environmental correlations}

The tetraploid cytotype of $R$. kuepferi exhibits an extended biogeographical and altitudinal distribution (Cosendai and Hörandl 2010; Schinkel et al. 2016; Kirchheimer et al. 2016). Kirchheimer et al. (2016) showed that the niche optimum of tetraploids is shifted to cooler conditions compared to diploids, which may have developed due to changes in the reproductive system of their originally warm-adapted diploid progenitors during postglacial re-colonization of higher regions in the Alps. Further explanation regards auto-polyploidization and hence maybe increased physiological tolerance due to whole genome duplication. Alpine habitats are harsh for plant life as they are characterized by low temperatures (including freezing), short vegetation periods and strong wind as well as UV-radiation exposure (Nagy and Grabherr 2009). Tolerance to cooler conditions allowed tetraploids to surmount high elevation barriers and establish new populations throughout a greater distribution range (Kirchheimer et al. 2018).

Values of Moran's / around zero among di- and tetraploids indicate a predominantly random distribution of methylation patterns without signs of spatial auto-correlation on global scale. The entirely diploid populations are geographically very close and have a smaller altitudinal range than tetraploid populations. Sexuality as well as physical proximity could have resulted in a greater exchange between adjacent populations. However, although the diploid sexuals 
show a distinct geographical structuring into four genetic groups (Cosendai et al. 2013), we can also assume that the overall warmer niche in the southwestern Alps contributed to a more homogeneous epigenetic pattern. On the other hand, tetraploids likewise show no relevant geographic structuring, neither in genetic patterns (Cosendai et al. 2013), nor in our methylation patterns. This in turn could be related to rapid colonization of the Alps and the often high degree of facultative sexuality. The presumption of isolation-by-distance could be ruled out in view of the high genetic diversity in unmethylated epiloci. According to our results so far (see Schinkel et al. 2016, 2017), tetraploid individuals could have emerged randomly from diploids by a triploid bridge and spread over the higher elevated areas of the central and eastern Alps. Multiple origins, a rapid colonization and regular intermixing due to facultative sexuality among tetraploids could have facilitated the inheritance of methylation patterns. Extreme cold conditions could have then facilitated the differentiation of mixed and obligate apomictic tetraploids.

We found high values of Moran's I (>0.5) in correlation to altitude, as well as to annual mean temperature and to annual precipitation among di- and tetraploid populations. We found greater variation in terms of temperature, but a larger overall impact of altitude on methylation patterns. Among the geographically close diploid populations in south-western France the altitudinal range is smaller compared to tetraploid populations, which is why an even larger Moran's / was found. On the other hand, Moran's / in correlation with annual precipitation is slightly lower because south-west France has a relatively warm and dry climate. Thus, calculated correlations suggest effects of climatic conditions on methylation patterns. Results support a hypothesis that changes of methylation patterns in tetraploids play a role in acclimation to colder climates. Whether acclimation is due to high phenotypic plasticity or an adaptation over generations needs to be studied. However, the consistent pattern in tetraploids over a large geographical area support a hypothesis of transgenerational inheritance and an adaptive role of methylation patterns. 


\section{References}

Adams KL and Wendel JF. 2005. Polyploidy and genome evolution in plants. Current Opinion in Plant Biology 8:135-141

Aliyu OM, Schranz ME and Sharbel TF. 2010. Quantitative variation for apomictic reproduction in the genus Boechera (Brassicaceae). American Journal of Botany 97:1719-1731

Alonso C, Pérez R, Bazaga P, Medrano M and Herrera CM. 2016. MSAP markers and global cytosine methylation in plants: a literature survey and comparative analysis for a wild-growing species. Molecular Ecology Resources 16:80-90

Arrigo N, Tuszynski JW, Ehrich D, Gerdes T and Alvarez N. 2009. Evaluating the impact of scoring parameters on the structure of intra-specific genetic variation using RawGeno, an $\mathrm{R}$ package for automating AFLP scoring. BMC Bioinformatics 10:10-33

Asker SE and Jerling L. 1992. Apomixis in Plants. CRC Press, Boca Raton

Baidouri ME, Kim KD, Abernathy B, Li Y-H, Qiu L-J and Jackson SA. 2018. Genic C-Methylation in Soybean Is Associated with Gene Paralogs Relocated to Transposable Element-Rich Pericentromeres. Molecular Plant 11(3):485-495

Banerjee A, Wani SH and Roychoudhury A. 2017. Epigenetic Control of Plant Cold Responses. Frontiers in Plant Science 8:1643

Banta JA and Richards CL. 2018. Quantitative epigenetics and evolution. Heredity 121:210-224

Barke H, Daubert M and HörandI E. 2018. Establishment of apomixis in diploid F2 hybrids and inheritance of apospory from F1 to F2 hybrids of the Ranunculus auricomus complex. Frontiers in Plant Science 9:1111

Baubec T, Dinh HQ, Pecinka A, Rakic B, Rozhon W, Wohlrab B, von Haeseler A and Scheid OM. 2010. Corporation of multiple chromatin modifications can generate unanticipated stability of epigenetic states in Arabidopsis. The Plant Cell 22:34-47

Becker C, Hagmann J, Müller J, Koenig D, Stegle O, Bogwardt K and Weigel D. 2011. Spontaneous epigenetic variation in the Arabidopsis thaliana methylome. Nature 480:245-250

Belanger FC and Hepburn AG. 1990. The evolution of CpNpG methylation in plants. Molecular Ecology 30:26-35

Bender J. 2004. DNA methylation and epigenetics. Annual Review of Plant Biology 55:41-68

Berger SL. 2007. The complex language of chromatin regulation during transcription. Nature 447:407-412

Bicknell RA. 1997. Isolation of a diploid, apomictic plant of Hieracium aurantiacum. Sexual Plant Reproduction 10(3):168-172

Bierzychudek P. 1985. Patterns in plant parthenogenesis. Experientia 41:1255-1264

Bird A. 2002. DNA methylation patterns and epigenetic memory. Genes \& Development 16:6-21

Bird A. 2007. Perceptions of epigenetics. Nature 447:396-398

Bomblies K, Higgins JD and Yant L. 2015. Meiosis evolves: adaptation to external and internal environments. New Phytologist 208:306-323 
Bossdorf O, Arcuri D, Richards CL and Pigliucci M. 2010. Experimental alteration of DNA methylation affects the phenotypic plasticity of ecologically relevant traits in Arabidopsis thaliana. Evolutionary Ecology 24:541-553

Boyko A and Kovalchuk I. 2008. Epigenetic control of plant stress response. Environmental and Molecular Mutagenesis 49:61-72

Boyko A, Blevins T, Yao YL, Golubov A, Bilichak A, Ilnytskyy Y, Hollander J, Meins F and Kovalchuk I. 2010. Transgenerational adaptation of Arabidopsis to stress requires DNA methylation and the function of Dicer-like proteins. PLOS ONE 5(4):10.1371/annotation/726f31b5-99c4-44e9-9cd6b8d66b3f6o38

Burnier J, Buerki S, Arrigo N, Küpfer P and Alvarez N. 2009. Genetic structure and evolution of Alpine polyploid complexes: Ranunculus kuepferi (Ranunculaceae) as a case study. Molecular Ecology 18:3730-3744

Carman JG. 1997. Asynchronous expression of duplicate genes in angiosperms may cause apomixis, bispory, tetraspory, and polyembryony. Biological Journal of the Linnean Society 61:51-94

Chinnusamy V and Zhu J-K. 2009. Epigenetic regulation of stress responses in plants. Current Opinion in Plant Biology 12(2):133-139

Cifuentes M, Grandont L, Moore G, Chèvre AM and Jenczewski E. 2009. Genetic regulation of meiosis in polyploid species: new insights into an old question. New Phytologist 186:29-36

Comai L. 2005. The advantages and disadvantages of being polyploid. Nature Reviews Genetics 6:836-846

Cosendai A-C and Hörandl E. 2010. Cytotype stability, facultative apomixis and Geographical Parthenogenesis in Ranunculus kuepferi (Ranunculaceae). Annals of Botany 105:457-470

Cosendai A-C, Rodewald J and HörandI E. 2011. Origin and distribution of autopolyploids via apomixis in the alpine species Ranunculus kuepferi (Ranunculaceae). Taxon 60:355-364

Cosendai A-C, Wagner J, Ladinig U, Rosche C and Hörandl E. 2013. Geographical parthenogenesis and population genetic structure in the alpine species Ranunculus kuepferi (Ranunculaceae). Heredity 110:560-569

De Storme N and Geelen D. 2013. Sexual Polyploidization in Plants: Cytological Mechanisms and Molecular Regulation. New Phytologist 198:670-684

De Storme N and Geelen D. 2013. Cytokinesis in plant male meiosis. Plant Signaling \& Behavior 8(3): 223394

De Storme N, Copenhaver GP and Geelen D. 2012. Production of diploid male gametes in Arabidopsis by cold-induced destabilization of postmeiotic radial microtubule arrays. Plant Physiology 160:18081826

De Storme N, Zamariola L, Mau M, Sharbel TF and Geelen D. 2013. Volume-based pollen size analysis: an advanced method to assess somatic and gametophytic ploidy in flowering plants. Plant Reproduction 26:65-81 
Dobeš C, Koch M and Sharbel TF. 2006. Embryology, karyology, and modes of reproduction in the north american genus Boechera (Brassicaceae): a compilation of seven decades of research. Annals of the Missouri Botanical Garden 93:517-534

Dobeš C, Mitchell-Olds T and Koch M. 2004. Extensive chloroplast haplotype variation indicates Pleistocene hybridization and radiation of North American Arabis drummondii, A. x divaricarpa, and A. holboellii (Brassicaceae). Molecular Ecology 13:349-370

Dong ZY, Wang YM, Zhang ZJ, Shen Y, Lin XY, Ou XF, Han FP and Liu B. 2006. Extent and pattern of DNA methylation alteration in rice lines derived from introgressive hybridization of rice and $Z i$ zania latifolia Griseb. Theoretical and Applied Genetics 113:196-205

Dowen RH, Pelizzola M, Schmitz RJ, Lister R, Dowen JM, Nery JR and Ecker JR. 2012. Widespread dynamic DNA methylation in response to biotic stress. Proceedings of the National Academy of Sciences of the United States of America. 109:e2183-e2191

Eckardt NA. 2003. Patterns of Gene Expression in Apomixis. The Plant Cell 15:1499-1502

Ecker S, Pancaldi V, Valencia A, Beck S and Paul DS. 2018. Epigenetic and Transcriptional Variability Shape Phenotypic Plasticity. Bioessays 40:1700148

Espinas NA, Saze H and Saijo Y. 2016. Epigenetic Control of Defense Signaling and Priming in Plants. Frontiers in Plant Science 7:1201

Excoffier $L$ and Lischer HE. 2010. Arlequin suite ver 3.5: a new series of programs to perform population genetics analyses under Linux and Windows. Molecular Ecology Resources 10:564-567

Feil R and Fraga MF. 2012. Epigenetics and the environment: emerging patterns and implications. Nature Reviews Genetics 13:97-109

Felitti SA, Seijo JG, González AM, Podio M, Laspina NV, Siena L, Ortiz JPA and Pessino SC. 2011. Expression of LORELEI-like genes in aposporous and sexual Paspalum notatum plants. Plant Molecular Biology 77:337-354

Feng S, Cokus SJ, Zhang X, Chen P-Y, Bostick M, Goll MG, Hetzel J, Jain J, Strauss SH, Halpern ME, Ukomadu C, Sadler KC, Pradhan S, Pellegrini M and Jacobsen SE. 2010. Conservation and divergence of methylation patterning in plants and animals. Proceedings of the National Academy of Sciences of the United States of America. 107:8689-8694

Finnegan EJ. 2010. DNA methylation: a dynamic regulator of genome organization and gene expression in plants. In: Pua E-C, Davey MR (eds) Plant Developmental Biology - Biotechnological Perspectives. Springer, Berlin, pp 295-323

Frankham R, Ballou JD and Briscoe DA. 2010. Introduction to Conservation Genetics. Cambridge University Press, Cambridge

Gao L, Diarso M, Zhang A, Zhang H, Dong Y, Liu L, Lv Z and Liu B. 2016. Heritable alteration of DNA methylation induced by whole-chromosome aneuploidy in wheat. New Phytologist 209:364-375

Gent JI, Ellis NA, Guo L, Harkess AE, Yao Y, Zhang X and Dawe RK. 2013. CHH islands: de novo DNA methylation in near-gene chromatin regulation in maize. Genome Research 23(4):628-637 
Gouil Q and Baulcombe DC. 2016. DNA Methylation Signatures of the Plant Chrmomethyltransferases. PLOS Genetics 12(12):e1006526

Grant-Downton RT and Dickinson HG. 2005. Epigenetics and its implications for plant biology: 1. The epigenetic network in plants. Annals of Botany 96:1143-1164

Gregor T. 2013. Apomicts in the vegetation of central Europe. Tuexenia 33:233-257

Grimanelli D. 2012. Epigenetic regulation of reproductive development and the emergence of apomixis in angiosperms. Current Opinion in Plant Biology 15:57-62

Grossniklaus U, Nogler GA and van Dijk PJ. 2001. How to avoid sex: the genetic control of gametophytic apomixis. The Plant Cell 13:1491-1498

Gruenbaum Y, Navehmany T, Cedar H and Razin A. 1981. Sequence specificity of methylation in higher plant DNA. Nature 292:860-862

Hand ML and Koltunow AM. 2014. The Genetic Control of Apomixis: Asexual Seed Formation. Genetics 197:441-450

Herman JJ and Sultan SE. 2016. DNA methylation mediates genetic variation for adaptive transgenerational plasticity. Proceedings of the Royal Society B: Biological Sciences 283(1838):20160988

Herrera CM and Bazaga P. 2013. Epigenetic correlates of plant phenotypic plasticity: DNA methylation differs between prickly and nonprickly leaves in heterophyllous Ilex aquifolium (Aquifoliaceae) trees. Botanical Journal of the Linnean Society 171:441-452

Hijmans RJ, Cameron SE, Parra JL, Jones PG and Jarvis A. 2005. Very high resolution interpolated climate surfaces for global land areas. International Journal of Climatology 25:1965-1978

Hojsgaard D, Klatt S, Baier R, Carman JG and Hörandl E. 2014. Taxonomy and Biogeography of Apomixis in Angiosperms and Associated Biodiversity Characteristics. Critical Reviews in Plant Sciences $33: 414-427$

Hörandl E. 2006. The complex causality of Geographical Parthenogenesis. New Phytologist 171(3):525-538

Hörandl E. 2011. Evolution and biogeography of alpine apomictic plants. Taxon 60:390-402

Hörandl E and Hadacek F. 2013. The oxidative damage initiation hypothesis of meiosis. Plant Reproduction 26:351-367

Jaenisch R and Bird A. 2003. Epigenetic regulation of gene expression: how the genome intergrates intrinsic and environmental signals. Nature Genetics 33:245-254

Johannes F, Porscher E, Teixeira FK, Saliba-Colombani V, Simon M, Agier N, Bulski A, Albuisson J, Heredia F, Audigier P, Bouchez D, Dillmann C, Guerche P, Hospital F and Colot V. 2009. Assessing the impact of transgenerational epigenetic variation on complex traits. PLOS Genetics 5:e1000530

Jones PA. 2012. Functions of DNA methylation: islands, start sites, gene bodies and beyond. Nature Reviews Genetics 13:484-492 
Kakunati T, Munakata K, Richards EJ and Hirochika H. 1999. Meiotically and mitotically stable inheritance of DNA hypomethylation induced by ddmı mutation of Arabidopsis thaliana. Genetics 151:831-838

Kantama L, Sharbel TF, Schranz ME, Mitchell-Olds T, de Vries SC and de Jong JHSGM. 2007. Diploid apomicts of the Boechera holboelli complex display large-scale chromosome substitutions and aberrant chromosomes. Proceedings of the National Academy of Sciences of the United States of America. 104:14026-14031

Kearney M. 2005. Hybridization, glaciation and Geographical Parthenogenesis. Trends in Ecology \& Evolution 20:495-502

Kim M, Kondo T, Takada I, Youn MY, Yamamoto Y, Takahashi S, Matsumoto T, Fujiyama S, Shirode Y and Yamaoka I. 2009. DNA demethylation in hormone-induced transcriptional derepression. Nature 461:1007-1012

Kinoshita T and Seki M. 2014. Epigenetic memory for stress response and adaptation in plants. Plant \& Cell Physiology 55:1859-1863

Kirchheimer B, Schinkel CCF, Dellinger AS, Klatt S, Moser D, Winkler M, Lenoir J, Caccianiga M, Guisan A, Nieto-Lugilde D, Svenning J-C, Thuiller W, Vittoz P, Willner W, Zimmermann NE, Hörandl E and Dullinger S. 2016. A matter of scale: apparent niche differentiation of diploid and tetraploid plants may depend on extent and grain of analysis. Journal of Biogeography 43:716-726

Kirchheimer B, Wessely J, Gattringer A, Hülber K, Moser D, Schinkel CCF, Appelhans M, Klatt S, Caccianiga M, Dellinger A, Guisan A, Kuttner M, Lenoir J, Maiorano L, Nieto-Lugilde D, Plutzar C, Svenning J-C, Willner W, Hörandl E and Dullinger S. 2018. Reconstructing Geographical Parthenogenesis: effects of niche differentiation and reproductive mode on Holocene range expansion of an alpine plant. Ecology Letters 21:392-401

Klatt S, Hadacek F, Hodač L, Brinkmann G, Eilerts M, Hojsgaard D and Hörandl E. 2016. Photoperiod extension enhances sexual megaspore formation and triggers metabolic reprogramming in facultative apomictic Ranunculus auricomus. Frontiers in Plant Science 7:278-293

Klatt S, Schinkel CCF, Kirchheimer B, Dullinger S and Hörandl E. 2018. Effects of cold treatments on fitness and mode of reproduction in the diploid and polyploid alpine plant Ranunculus kuepferi (Ranunculaceae). Annals of Botany 121(8):1287-1298

Koltunow AM and Grossniklaus U. 2003. Apomixis: a developmental perspective. Annual Review of Plant Biology 54:547-574

Kovařik A, Matyášek R, Leitch A, Gazdová B, Fulneček J and Bezděk M. 1997. Variability in CpNpG methylation in higher plant genomes. Gene 204:25-33

Küpfer P. 1974. Recherches sur les liens de parente entre la flore orophile des Alpes et celle des Pyrenees. Boissiera 23:1-322 
Labra M, Ghiani A, Citterio S, Sgorbati S, Sala F, Vannini C, Ruffini-Castiglione M and Bracale M. 2002. Analysis of cytosine methylation pattern in response to water deficit in pea root tips. Plant Biology 4:694-699

Laspina NV, Vega T, Seijo JG, González AM, Martelotto LG, Stein J, Podio M, Ortiz JPA, Echenique VC, Quarin CL and Pessino SC. 2008. Gene expression analysis at the onset of aposporous apomixis in Paspalum notatum. Plant Molecular Biology 67:615-628

Latzel V and Klimesova J. 2010. Transgenerational plasticity in clonal plants. Evolutionary Ecology 24:1537-1543

Law JA and Jacobsen SE. 2010. Establishing, maintaining and modifying DNA methylation patterns in plants and animals. Nature Reviews Genetics 11:204-220

Levin DA. 1975. Minority cytotype exclusion in local plant populations. Taxon 24:35-43

Levin DA. 2003. Ecological speciation: Lessons from invasive species. Systematic Botany 28:643-650

Li A, Hu B-Q, Xue Z-Y, Chen L, Wang W-X, Song W-Q, Chen C-B and Wang C-G. 2011. DNA methylation in genomes of several annual herbaceous and woody perennial plants of varying ploidy as detected by MSAP. Plant Molecular Biology Reporter 29:784-793

Lippman Z, Gendrel AV, Black M, Vaughn MW, Dedhia N, McCombie WR, Lavine K, Mittal V, May B, Kasschau KD, Carrington JC, Doerge RW, Colot V and Martienssen R. 2004. Role of transposable elements in heterochromatin and epigenetic control. Nature 430:471-476

Liu T, Li Y, Duan W, Huang F and Hou X. 2017. Cold acclimation alters DNA methylation patterns and confers tolerance to heat and increases growth rate in Brassica rapa. Journal of Experimental Botany 68(5):1213-1224

Madlung A and Wendel JF. 2013. Genetic and Epigenetic Aspects of Polyploid Evolution in Plants. Cytogenetic and Genome Research 140:270-285

Massicotte R and Angers B. 2012. General-purpose genotype or how epigenetics extend the flexibility of a genotype. Genetics Research International 2012:1-7

Matzke MA, Kanno T and Matzke AJM. 2015. RNA-Directed DNA Methylation: The Evolution of a Complex Epigenetic Pathway in Flowering Plants. Annual Review of Plant Biology 66:243-267

Merotto A Jr, Goulart ICGR, Nunes AL, Kalsing A, Markus C, Menezes VG and Wander AE. 2016. Evolutionary and social consequences of introgression of non-transgenic herbicide resistance from rice to weedy rice in Brazil. Evolutionary Applications 9:837-846

Messeguer R, Ganal MW, Steffens JC and Tanksley SD. 1991. Characterization of the level, target sites and inheritance of cytosine methylation in tomato nuclear DNA. Plant Molecular Biology 16:753770

Mittelsten SO, Afsar K and Paszkowski J. 2003. Formation of stable epialleles and their paramutationlike interaction in tetraploid Arabidopsis thaliana. Nature Genetics 34:450-454

Miura A, Yonebayashi S, Watanabe K, Toyama T, Shimada H and Kakutani T. 2001. Mobilization of transposons by a mutation abolishing full DNA methylation in Arabidopsis. Nature 411:212-214 
Nagy L and Grabherr G. 2009. The biology of alpine habitats. Oxford University Press, New York

Nogler GA. 1984. Gametophytic apomixis. In: Johri PBM (eds) Embryology of angiosperms. Springer, Berlin, $p p$ 475-518

Oksanen J, Guillaume BF, Friendly M, Kindt R, Legendre P, McGlinn D, Minchin PR, O'Hara RB, Simpson GL, Stevens MHH, Szoecs E and Wagner H. 2017. vegan: Community Ecology Package.

Otto SP and Whitton J. 2000. Polyploid Incidence and Evolution. Annual Review of Genetics 34:401-437

Ozias-Akins P and Van Dijk PJ. 2007. Mendelian genetics of apomixis in plants. Annual Review of Genetics 41:509-537

Paszkowski J and Grossniklaus U. 2011. Selected aspects of transgenerational epigenetic inheritance and resetting in plants. Current Opinion in Plant Biology 14(2):195-203

Paun O, Bateman RM, Fay MF, Hedrén M, Civeyrel L and Chase MW. 2010. Stable Epigenetic Effects Impact Adaptation in Allopolyploid Orchids (Dactylorhiza: Orchidaceae). Molecular Biology and Evolution 27(11):2465-2473

Paun O, Stuetty TF and HörandI E. 2006. The role of hybridization, polyploidization and glaciation in the origin and evolution of the apomictic Ranunculus cassubicus complex. New Phytologist 171:223236

Pellino M, Hojsgaard D, Schmutzer T, Scholz U, Hörandl E, Vogel H and Sharbel TF. 2013. Asexual genome evolution in apomictic Ranunculus auricomus complex: examining the effects of hybridization and mutation accumulation. Molecular Ecology 22:5908-5921

Pikaard CS and Scheid OM. 2014. Epigenetic Regulation in Plants. Cold Spring Harbor Perspectives Biology 6(12):a019315

Podio M, Felitti SA, Siena LA, Delgado L, Mancini M, Seijo JG, González AM, Pessino SC and Ortiz JPA. 2014. Characterization and expression analysis of SOMATIC EMBRYOGENESIS RECEPTOR KINASE (SERK) genes in sexual and apomictic Paspalum notatum. Plant Molecular Biology 84:479495

Polegri L, Calderini O, Arcioni S and Pupilli F. 2010. Specific expression of apomixis-linked alleles revealed by comparative transcriptomic analysis of sexual and apomictic Paspalum simplex Morong flowers. Journal of Experimental Botany 61(6):1869-1883

QGIS Development Team. 2018. QGIS Geographic Information System. Open Source Geospatial Foundation

R Core Team. 2017. R: A language and environment for statistical computing. R Foundation for Statistical Computing

Ramsey J and Schemske DW. 1998. Pathways, mechanisms, and rates of polyploid formation in flowering plants. Annual Review of Ecology and Systematics 29:467-501

Richards EJ. 2006. Inherited epigenetic variation-revisiting soft inheritance. Nature Reviews 7:395-401

Rodrigo JM, Zappacosta DC, Selva JP, Garbus I, Albertini E and Echenique V. 2017. Apomixis frequency under stress conditions in weeping lovegrass (Eragrostis curvula). PLOS ONE 12(4):e0175852 
Ronceret A and Vielle-Calzada JP. 2015. Meiosis, unreduced gametes, and parthenogenesis: implications for engineering clonal seed formation in crops. Plant Reproduction 28:91-102

Santos F, Hendrich B, Reik W and Dean W. 2002. Dynamic reprogramming of DNA methylation in the early mouse embryo. Developmental Biology 241:172-182

Schinkel CCF, Kirchheimer B, Dellinger AS, Klatt S, Winkler M, Dullinger S and Hörandl E. 2016. Correlations of polyploidy and apomixis with elevation and associated environmental gradients in an alpine plant. AoB PLANTS 8:plwo64

Schinkel CCF, Kirchheimer B, Dullinger S, Geelen D, De Storme N and Hörandl E. 2017. Pathways to polyploidy: indications of a female triploid bridge in the alpine species Ranunculus kuepferi (Ranunculaceae). Plant Systematics and Evolution 303:1093-1108

Schulz B, Eckstein RL and Durka W. 2013. Scoring and analysis of methylation-sensitive amplification polymorphisms for epigenetic population studies. Molecular Ecology Resources 13:642-653

Shah JN, Kirioukhova O, Pawar P, Tayyab M, Mateo JL and Johnston AJ. 2016. Depletion of Key Meiotic Genes and Transcriptome-Wide Abiotic Stress Reprogramming Mark Early Preparatory Events Ahead of Apomeiotic Transition. Frontiers in Plant Science 7:1539

Shan X, Wang X, Yang G, Wu Y, Su S, Li S, Liu H and Yuan Y. 2013. Analysis of the DNA methylation of maize (Zea mays L.) in response to cold stress based on methylation-sensitive amplified polymorphisms. Journal of Plant Biology 56:32-38

Sherman JD and Talbert LE. 2002. Vernalization-induced changes of DNA methylation pattern in winter wheat. Genome 45:253-260

Siena LA, Sartor ME, Espinoza F, Quarin CL and Ortiz JPA. 2008. Genetic and embryological evidences of apomixis at the diploid level in Paspalum rufum support recurrent auto-polyploidization in the species. Sexual Plant Reproduction 21:205-215

Slotkin RK, Vaughn M, Borges F, Tanunrdzic M, Becker JD, Feijo JA and Martienssen RA. 2009. Epigenetic reprogramming and small RNA silencing of transposable elements in pollen. Cell 136:561472

Song $\mathrm{Q}$ and Chen ZJ. 2016. Epigenetic and developmental regulation in plant polyploids. Current Opinion in Plant Biology 24:101-109

Song Y, Liu L, Feng Y, Wei Y, Yue X, He W and An L. 2015. Chilling- and Freezing-Induced Alterations in Cytosine Methylation and Its Association with the Cold Tolerance of an Alpine Subnival Plant, Chorispora bungeana. PLOS ONE 10(8):e0135485

Stroud H, Do T, Du J, Zhong X, Feng S, Johnson L, Patel DJ and Jacobsen SE. 2014. Non-CG methylation patterns shape the epigenetic landscape in Arabidopsis. Nature Structural \& Molecular Biology 21:64-72 
Stucki S, Orozco-terWengel P, Forester BR, Duruz S, Colli L, Masembe C, Negrini R, Landguth E, Jones MR, Bruford MW, Taberlet P and Joost S. 2017. High performance computation of landscape genomic models including local indicators of spatial association. Molecular Ecology Resources 17:1072-1089

Takuno S and Gaut BS. 2013. Gene body methylation is conserved between plant orthologs and is of evolutionary consequence. Proceedings of the National Academy of Sciences of the United States of America. 110(5):1797-1802

Takuno S, Ran J-H and Gaut BS. 2016. Evolutionary patterns of genic DNA methylation vary across land plants. Nature Plants 2:15222

Teixeira FK and Colot V. 2009. Gene body DNA methylation in plants: a means to an end or an end to a means?. The EMBO Journal 28(8):997-998

Vanyushin BF and Ashapkin VV. 2011. DNA methylation in higher plants: Past, present and future. Biochimica et Biophysica Acta (BBA) - Gene Regulatory Mechanisms 1809(8):360-368

Vaughn MW, Tanurdzic M, Lippman Z, Jiang H, Carrasquillo R, Rabinowicz PD, Dedhia N, McCombie WR, Agier N, Bulski A, Colot V, Doerge RW and Martienssen RA. 2007. Epigenetic natural variation in Arabidopsis thaliana. PLOS Biology 5:1617-1629

Verhoeven KJF and Preite V. 2013. Epigenetic variation in asexually reproducing organisms. Evolution 68:644-655

Verhoeven KJF, Jansen JJ, van Dijk PJ and Biere A. 2010. Stress-induced DNA methylation changes and their heritability in asexual dandelions. New Phytologist 185:1108-18

Verhoeven KJF, von Holdt MB and Sork VL. 2016. Epigenetics in ecology and evolution: what we know and what we need to know. Molecular Ecology 25:1631-1638

Wickham H. 2009. ggplot2: Elegant Graphics for Data Analysis. Springer, New York

Yan H, Kikuchi S, Neumann P, Zhang W, Wu Y, Chen F and Jiang J. 2010. Genome-wide mapping of cytosine methylation revealed dynamic DNA methylation patterns associated with genes and centromeres in rice. The Plant Journal 63:353-365

Zemach A, Kim MY, Hsieh P-H, Coleman-Derr D, Eshed-Williams L, Thao K, Harmer SL and Zilberman D. 2013. The Arabidopsis Nucleosome Remodeler DDM1 Allows DNA Methyltransferases to Access H1-Containing Heterochromatin. Cell 153:193-205

Zhang J, Liua Y, Xiaa E-H, Yaoa Q-Y, Liud X-D and Gaoa L-Z. 2015. Autotetraploid rice methylome analysis reveals methylation variation of transposable elements and their effects on gene expression. Proceedings of the National Academy of Sciences of the United States of America. 112(50):7022-7029

Zhong S, Fei Z, Chen Y-R, Zheng Y, Huang M, Vrebalov J, McQuinn R, Gapper N, Liu B, Xiang J, Shao $Y$ and Giovannoni JJ. 2013. Single-base resolution methylomes of tomato fruit development reveal epigenome modifications associated with ripening. Nature Biotechnology 31:154-159 
Zilberman D, Gehring M, Tran RK, Ballinger T and Henikoff S. 2007. Genome-wide analysis of Arabidopsis thaliana DNA methylation uncovers an independence between methylation and transcription. Nature Genetics 39:61-69 


\section{Appendix}

A1. List of the 123 individuals used for MSAP analyses with provenances, ploidy level and respective mode of reproduction (after Schinkel et al. 2016).

\begin{tabular}{|c|c|c|c|c|c|}
\hline \multirow[b]{2}{*}{ Individual } & \multicolumn{2}{|c|}{ Geographic Coordinates (WGS84) } & \multirow[b]{2}{*}{ Altitude [m a.s.l.] } & \multirow[b]{2}{*}{ Cytotype } & \multirow[b]{2}{*}{ Reproduction mode } \\
\hline & North & East & & & \\
\hline $14-^{2}{ }^{2}$ & $44 \cdot 35489$ & 6.505583 & 1880 & diploid & mixed* \\
\hline 26_2_1 & 44.90033 & 5.469 & 1456 & diploid & mixed* \\
\hline 31_3_2 & 44.19522 & 7.659222 & 1937 & diploid & mixed* \\
\hline $32-2 \_3$ & 44.20667 & 7.1475 & 2320 & diploid & mixed* \\
\hline 204_1_1 & 44.15725 & 6.729195 & 1640 & diploid & mixed* \\
\hline 207_3_1 & $44 \cdot 30108$ & 6.567389 & 1921 & diploid & mixed* \\
\hline 3_3_1 & 44.20028 & 7.15639 & 2291 & diploid & obligate sexual \\
\hline $3-3{ }^{2} 2$ & 44.20028 & 7.15639 & 2291 & diploid & obligate sexual \\
\hline $23-2 \_1$ & $43 \cdot 74931$ & 6.657444 & 1616 & diploid & obligate sexual \\
\hline $23{ }^{2}{ }^{2} 2$ & $43 \cdot 74931$ & 6.657444 & 1616 & diploid & obligate sexual \\
\hline 24_3_1 & 44.15083 & 6.543611 & 1925 & diploid & obligate sexual \\
\hline $24-4{ }^{2}$ & 44.15083 & 6.543611 & 1925 & diploid & obligate sexual \\
\hline 25_2_1 & 44.90128 & 5.476167 & 1435 & diploid & obligate sexual \\
\hline 25_3_3 & 44.90128 & $5 \cdot 476167$ & 1435 & diploid & obligate sexual \\
\hline 25_4_3 & 44.90128 & 5.476167 & 1435 & diploid & obligate sexual \\
\hline 26_3_2 & 44.90033 & $5 \cdot 469$ & 1456 & diploid & obligate sexual \\
\hline 27_1_1 & 44.83933 & $5 \cdot 424222$ & 1449 & diploid & obligate sexual \\
\hline $27-2 \_1$ & 44.83933 & $5 \cdot 424222$ & 1449 & diploid & obligate sexual \\
\hline 27_2_3 & 44.83933 & 5.424222 & 1449 & diploid & obligate sexual \\
\hline 29_1_1 & 44.23111 & 7.617222 & 2020 & diploid & obligate sexual \\
\hline $29 \_2 \_3$ & 44.23111 & 7.617222 & 2020 & diploid & obligate sexual \\
\hline 31_2_3 & 44.19522 & 7.659222 & 1937 & diploid & obligate sexual \\
\hline 33-3_1 & 44.21305 & 7.146667 & 2328 & diploid & obligate sexual \\
\hline 33_3_3 & 44.21305 & 7.146667 & 2328 & diploid & obligate sexual \\
\hline 112_1_2 & 43.8525 & 6.352778 & 1626 & diploid & obligate sexual \\
\hline $112 \_2 \_3$ & 43.8525 & 6.352778 & 1626 & diploid & obligate sexual \\
\hline 112_4_1 & 43.8525 & 6.352778 & 1626 & diploid & obligate sexual \\
\hline $114 \_4-3$ & 44.72089 & 6.919861 & 2339 & diploid & obligate sexual \\
\hline 115_3_2 & 44.245 & 6.756111 & 1891 & diploid & obligate sexual \\
\hline 115_3_3 & 44.245 & 6.756111 & 1891 & diploid & obligate sexual \\
\hline $117{ }^{2}{ }^{2} 2$ & 43.74556 & 6.655833 & 1632 & diploid & obligate sexual \\
\hline 117_3_3 & 43.74556 & 6.655833 & 1632 & diploid & obligate sexual \\
\hline
\end{tabular}




\begin{tabular}{|c|c|c|c|c|c|}
\hline 118_3_3 & 44.23161 & 7.628333 & 1636 & diploid & obligate sexual \\
\hline 201_2_2 & 44.08044 & 6.6375 & 2025 & diploid & obligate sexual \\
\hline 201_2_3 & 44.08044 & 6.6375 & 2025 & diploid & obligate sexual \\
\hline 202_1_1 & 44.159 & 6.714611 & 1829 & diploid & obligate sexual \\
\hline $202 \_4{ }^{2}$ & 44.159 & 6.714611 & 1829 & diploid & obligate sexual \\
\hline 203_1_3 & 44.16294 & 6.710805 & 1840 & diploid & obligate sexual \\
\hline 203_3_1 & 44.15725 & 6.729195 & 1840 & diploid & obligate sexual \\
\hline $204-4 \_2$ & 44.15725 & 6.729195 & 1640 & diploid & obligate sexual \\
\hline 206_1_2 & 44.2468 & 6.6988 & 2049 & diploid & obligate sexual \\
\hline 206_3_1 & 44.2468 & 6.6988 & 2049 & diploid & obligate sexual \\
\hline 207_1_3 & $44 \cdot 30108$ & 6.567389 & 1921 & diploid & obligate sexual \\
\hline 233_1_1 & 44.12946 & 6.96878 & 2185 & diploid & obligate sexual \\
\hline 233_1_2 & 44.12946 & 6.96878 & 2185 & diploid & obligate sexual \\
\hline 235_1_1 & 44.16689 & 6.70763 & 1930 & diploid & obligate sexual \\
\hline 235_1_3 & 44.16689 & 6.70763 & 1930 & diploid & obligate sexual \\
\hline 17_1_2 & 45.05069 & 6.390778 & 2357 & tetraploid & obligate apomictic \\
\hline $17-3 \_1$ & 45.05069 & 6.390778 & 2357 & tetraploid & obligate apomictic \\
\hline 17_4_3 & 45.05069 & 6.390778 & 2357 & tetraploid & obligate apomictic \\
\hline 20_1_2 & 46.34719 & 7.724111 & 2200 & tetraploid & obligate apomictic \\
\hline 20_2_1 & 46.34719 & 7.724111 & 2200 & tetraploid & obligate apomictic \\
\hline $20 \_4{ }^{2}$ & 46.34719 & $7 \cdot 724111$ & 2200 & tetraploid & obligate apomictic \\
\hline 36_1_3 & $45 \cdot 38542$ & 7.043722 & 2152 & tetraploid & obligate apomictic \\
\hline $36 \_3{ }^{2}$ & $45 \cdot 38542$ & 7.043722 & 2152 & tetraploid & obligate apomictic \\
\hline 48_1_1 & 46.47153 & 9.728889 & 2262 & tetraploid & obligate apomictic \\
\hline 48_1_3 & 46.47153 & 9.728889 & 2262 & tetraploid & obligate apomictic \\
\hline 48_4_1 & 46.47153 & 9.728889 & 2262 & tetraploid & obligate apomictic \\
\hline $54-^{2}-^{2}$ & 46.27239 & 10.57506 & 2303 & tetraploid & obligate apomictic \\
\hline 54_3_1 & 46.27239 & 10.57506 & 2303 & tetraploid & obligate apomictic \\
\hline 54_3_2 & 46.27239 & 10.57506 & 2303 & tetraploid & obligate apomictic \\
\hline $54-4 \_3$ & 46.27239 & 10.57506 & 2303 & tetraploid & obligate apomictic \\
\hline 75_1_2 & 46.52845 & 9.811194 & 2678 & tetraploid & obligate apomictic \\
\hline 79_1_2 & 46.98753 & 10.35919 & 2280 & tetraploid & obligate apomictic \\
\hline 79_2_2 & 46.98753 & 10.35919 & 2280 & tetraploid & obligate apomictic \\
\hline $83-3{ }^{2} 2$ & 47.0407 & 12.69105 & 2271 & tetraploid & obligate apomictic \\
\hline 104_1_3 & 46.45142 & 10.30158 & 2298 & tetraploid & obligate apomictic \\
\hline 104_2_1 & 46.45142 & $10.3015^{8}$ & 2298 & tetraploid & obligate apomictic \\
\hline 111_2_1 & 44.279 & 6.719222 & 2243 & tetraploid & obligate apomictic \\
\hline 111_3_1 & 44.279 & 6.719222 & 2243 & tetraploid & obligate apomictic \\
\hline
\end{tabular}




\begin{tabular}{|c|c|c|c|c|c|}
\hline 114_2_1 & 44.72089 & 6.919861 & 2339 & tetraploid & obligate apomictic \\
\hline 114_2_3 & 44.72089 & 6.919861 & 2339 & tetraploid & obligate apomictic \\
\hline 114_3_1 & 44.72089 & 6.919861 & 2339 & tetraploid & obligate apomictic \\
\hline $200 \_2 \_3$ & 44.18431 & 7.603472 & 1390 & tetraploid & obligate apomictic \\
\hline 205_1_3 & 44.23711 & 6.704778 & 2265 & tetraploid & obligate apomictic \\
\hline 235_2_1 & 44.16689 & 6.70763 & 1930 & tetraploid & obligate apomictic \\
\hline 81_1_10 & 46.98753 & 10.32303 & 2526 & tetraploid & obligate apomictic \\
\hline 81_1_12 & 46.98753 & 10.32303 & 2526 & tetraploid & obligate apomictic \\
\hline 4_4_2 & 44.17528 & 6.89806 & 2050 & tetraploid & mixed* \\
\hline 17_1_3 & 45.05069 & 6.390778 & 2357 & tetraploid & mixed* \\
\hline $17 \_2 \_3$ & 45.05069 & 6.390778 & 2357 & tetraploid & mixed* \\
\hline 17_3_2 & 45.05069 & 6.390778 & 2357 & tetraploid & mixed* \\
\hline $17-4-2$ & 45.05069 & 6.390778 & 2357 & tetraploid & mixed* \\
\hline 20_1_3 & 46.34719 & $7 \cdot 724111$ & 2200 & tetraploid & mixed* \\
\hline $34-^{2}-^{2}$ & $45 \cdot 24244$ & 6.951889 & 2120 & tetraploid & mixed* \\
\hline 36_1_1 & $45 \cdot 38542$ & 7.043722 & 2152 & tetraploid & mixed* \\
\hline 36_1_2 & $45 \cdot 38542$ & 7.043722 & 2152 & tetraploid & mixed* \\
\hline $36 \_4-1$ & $45 \cdot 38542$ & 7.043722 & 2152 & tetraploid & mixed* \\
\hline $36 \_4{ }^{2}$ & $45 \cdot 38542$ & 7.043722 & 2152 & tetraploid & mixed* \\
\hline 37_3_1 & $45 \cdot 61622$ & $7 \cdot 552611$ & 2115 & tetraploid & mixed* \\
\hline 37_3_2 & $45 \cdot 61622$ & $7 \cdot 55^{2611}$ & 2115 & tetraploid & mixed* \\
\hline 40_1_3 & 46.0825 & 7.0125 & 1860 & tetraploid & mixed* \\
\hline 40_2_1 & 46.0825 & 7.0125 & 1860 & tetraploid & mixed* \\
\hline 40_2_2 & 46.0825 & 7.0125 & 1860 & tetraploid & mixed* \\
\hline 45_1_3 & 46.57119 & 8.410556 & 2400 & tetraploid & mixed* \\
\hline 47_1_3 & 46.54628 & 9.211389 & 2211 & tetraploid & mixed* \\
\hline 48_1_2 & 46.47153 & 9.728889 & 2262 & tetraploid & mixed* \\
\hline 53_1_2 & 46.54856 & 10.43431 & 2456 & tetraploid & mixed* \\
\hline 54_1_1 & 46.27239 & 10.57506 & 2303 & tetraploid & mixed* \\
\hline 54_1_2 & 46.27239 & 10.57506 & 2303 & tetraploid & mixed* \\
\hline 54_2_1 & 46.27239 & 10.57506 & 2303 & tetraploid & mixed* \\
\hline 54_2_3 & 46.27239 & 10.57506 & 2303 & tetraploid & mixed* \\
\hline 54_4_1 & 46.27239 & 10.57506 & 2303 & tetraploid & mixed* \\
\hline $54-4{ }^{2}$ & 46.27239 & 10.57506 & 2303 & tetraploid & mixed* \\
\hline 58_1_1 & 46.45667 & 11.88814 & 2117 & tetraploid & mixed* \\
\hline 58_1_2 & 46.45667 & 11.88814 & 2117 & tetraploid & mixed* \\
\hline 58_1_3 & 46.45667 & 11.88814 & 2117 & tetraploid & mixed* \\
\hline $58 \_2 \_2$ & 46.45667 & 11.88814 & 2117 & tetraploid & mixed* \\
\hline
\end{tabular}




\begin{tabular}{llllll} 
58_2_3 & 46.45667 & 11.88814 & 2117 & tetraploid & mixed* \\
59_1_7 & 46.66434 & 12.18316 & 2391 & tetraploid & mixed* \\
73_1_3 & 47.21906 & 10.31961 & 2180 & tetraploid & mixed* \\
75_1_3 & 46.52845 & 9.811194 & 2678 & tetraploid & mixed* \\
77_3_1 & 46.36692 & 7.652778 & 2259 & tetraploid & mixed* \\
79_3_2 & 46.98753 & 10.35919 & 2280 & tetraploid & mixed* \\
79_4_1 & 46.98753 & 10.35919 & 2280 & tetraploid & mixed* \\
81_1_4 & 46.98753 & 10.32303 & 2526 & tetraploid & mixed* \\
81_1_5 & 46.98753 & 10.32303 & 2526 & tetraploid & mixed* \\
83_4_1 & 47.0407 & 12.69105 & 2271 & tetraploid & mixed* \\
114_3_3 & 44.72089 & 6.919861 & 2339 & tetraploid & mixed* \\
114_4_2 & 44.72089 & 6.919861 & 2339 & tetraploid & mixed* \\
204_2_3 & 44.15725 & 6.729195 & 1640 & tetraploid & mixed* \\
204_3_1 & 44.15725 & 6.729195 & 1640 & tetraploid & mixed* \\
205_4_1 & 44.23711 & 6.704778 & 2265 & tetraploid & mixed* \\
\hline
\end{tabular}

A2. DNA extraction, adapter and primer sequences DNA extraction

\begin{tabular}{|c|c|}
\hline Adaptor & Sequence \\
\hline EcoRIF & 5'- CTCGTAGACTGCGTACC \\
\hline EcoRIR & 5'- AATTGGTACGCAGTC \\
\hline Mspl/Hpall F & 5'- GATCATGAGTCCTGCT \\
\hline Mspl/Hpall R & 5'- CGAGCAGGACTCATGA \\
\hline Preselective Primer & Sequence \\
\hline EcoRI & $5^{\prime}-$ GACTGCGTACCAATTCA \\
\hline Mspl/Hpall & $5^{\prime}-\quad$ ATCATGAGTCCTGCTCGG \\
\hline Selective Primer & Sequence \\
\hline EcoRI & $5^{\prime}-$ GACTGCGTACCAATTCAAC \\
\hline Mspl/Hpall 1 & 5'- [FAM] - ATCATGAGTCCTGCTCGGCTCG \\
\hline Mspl/Hpall 2 & 5'- [HEX] - ATCATGAGTCCTGCTCGGCTGA \\
\hline Mspl/Hpall 3 & 5'- [NED] - ATCATGAGTCCTGCTCGGCATA \\
\hline
\end{tabular}


For standardized DNA preparation and high yields $1 \mathrm{~cm}^{2}$ leaf material per sample was disrupted using a OIAGEN TissueLyser II (OIAGEN, Hilden, Germany) with 2 steel beads (diameter: $5 \mathrm{~mm}$ ) in $2 \mathrm{ml}$ Eppendorf tubes, operating at $25 \mathrm{~Hz}$ for 2 mins. Isolation of DNA was realized with OIAGEN DNeasy Plant Mini Kit (QIAGEN, Hilden, Germany) using a slightly modified protocol. During lipid cleansing (step 2) a reduced amount of $360 \mu \mathrm{AP}_{1}$ Buffer was used while $40 \mu \mathrm{l}$ 2.6\% polyvinylpyrrolidone (PVP; Carl Roth GmbH \& Co. KG, Karlsruhe, Germany) solution was added to attenuate detrimental influence of high polyphenolic compound content in our samples (Healey et al. 2014). Incubation times for lipid cleansing and elution (step 3, step 12 and 13) were prolonged to 30 mins with preheated elution buffer. Elution of isolated DNA (step 12 and 13) was performed with reduced amount of elution buffer ( $50 \mu$ l each) to gain higher concentrations. Isolate quality and quantity were checked on $1.5 \%$ agarose gel (Carl Roth GmbH \& Co. KG, Karlsruhe, Germany) and NanoDrop 2000 (Thermo Fisher Scientific, Waltham, MA, USA). Only samples without impurities and a DNA content of at least $20 \mathrm{ng} / \mu \mathrm{l}$ were processed further.

A3. RawGeno input parameters and actuating factors

\begin{tabular}{|c|c|c|c|c|c|c|c|c|}
\hline \multirow[b]{2}{*}{ Dye } & \multicolumn{2}{|c|}{ Bin Size } & \multicolumn{2}{|c|}{ Thresholds } & \multicolumn{4}{|c|}{ Estimation } \\
\hline & Min. & Max. & RFU & Reprod. & $N_{\text {Bins }}$ & Reprod. & $E_{\text {Bonnin }}$ & Polymorph. \\
\hline Blue & 1.5 & 1.7 & 200 & 90 & 100 & 85.15 & $<0.01$ & 0.77 \\
\hline Green & 1.6 & 2 & 250 & 90 & 110 & 77.78 & $<0.00$ & 0.76 \\
\hline Yellow & 1.5 & 2 & 150 & 95 & 108 & 92.21 & $<0.01$ & 0.79 \\
\hline
\end{tabular}

A4. Geary's C values correlated to environmental variables

\begin{tabular}{lllll}
\hline Population & Group & Altitude & Mean Annual Temp. & Annual Precipitation \\
\hline 3 & $2 \times S$ & 2.550 & 2.577 & 2.232 \\
23 & $2 \times S$ & 0.676 & 1.869 & 2.464 \\
24 & $2 \times S$ & 0.081 & 0.055 & 0.021 \\
25 & $2 \times S$ & 2.164 & 0.366 & 0.001 \\
26 & $2 \times S$ & 1.948 & 0.279 & 0.021 \\
27 & $2 \times S$ & 2.019 & 0.464 & 0.010 \\
29 & $2 \times S$ & 0.391 & 0.378 & 0.072 \\
33 & $2 \times S$ & 2.992 & 3.090 & 2.613
\end{tabular}




\begin{tabular}{|c|c|c|c|c|}
\hline 31 & $2 \times S$ & 0.108 & 0.695 & 0.696 \\
\hline 233 & $2 \times S$ & 1.481 & 1.499 & 1.008 \\
\hline 235 & $2 \times S$ & 0.092 & 0.055 & 0.021 \\
\hline 202 & $2 \times S$ & 0.003 & 0.090 & 0.218 \\
\hline 203 & $2 \times S$ & 0.014 & 0.221 & 0.371 \\
\hline 204 & $2 \times S$ & 0.278 & 0.596 & 0.768 \\
\hline 206 & $2 \times S$ & 0.532 & 1.149 & 1.008 \\
\hline 201 & $2 \times S$ & 0.414 & 0.378 & 0.153 \\
\hline 207 & $2 \times S$ & 0.073 & 0.214 & 0.153 \\
\hline 112 & $2 \times S$ & 0.618 & 1.475 & 2.094 \\
\hline 115 & $2 \times S$ & 0.027 & 0.000 & 0.010 \\
\hline 117 & $2 \times S$ & 0.585 & 1.666 & 2.464 \\
\hline 26 & $2 \times M$ & 1.948 & 0.279 & 0.021 \\
\hline 31 & $2 \times M$ & 0.108 & 0.695 & 0.696 \\
\hline 204 & $2 \times M$ & 0.278 & 0.596 & 0.768 \\
\hline 207 & $2 \times M$ & 0.073 & 0.214 & 0.153 \\
\hline 201 & $4 \times M$ & 0.027 & 0.108 & 3.264 \\
\hline 36 & $4 \times M$ & $0.15^{8}$ & 0.404 & 0.288 \\
\hline 37 & $4 \times M$ & 0.332 & 0.336 & 0.342 \\
\hline 40 & $4 \times M$ & 3.285 & 1.067 & 0.609 \\
\hline 48 & $4 \times M$ & 0.018 & 0.108 & 0.010 \\
\hline 54 & $4 \times M$ & 0.112 & 0.108 & 2.012 \\
\hline 58 & $4 \times M$ & 0.321 & 0.057 & 1.876 \\
\hline 75 & $4 \times M$ & 4.632 & 3.252 & 0.860 \\
\hline 17 & $4 \times M$ & 0.356 & 0.016 & 0.535 \\
\hline 17 & $4 \times M$ & 0.356 & 0.016 & 0.535 \\
\hline 79 & $4 \times M$ & 0.050 & 2.140 & 0.116 \\
\hline 83 & $4 \times M$ & 0.032 & 0.612 & 0.060 \\
\hline 81 & $4 \times M$ & 2.004 & 2.140 & 0.152 \\
\hline 204 & $4 \times M$ & 8.287 & $7 \cdot 480$ & 1.618 \\
\hline 205 & $4 \times M$ & 0.023 & 0.562 & 0.022 \\
\hline
\end{tabular}




\begin{tabular}{lllll}
114 & $4 \times \mathrm{M}$ & 0.259 & 0.124 & 0.059 \\
20 & $4 \times A$ & 0.027 & 0.108 & 3.264 \\
36 & $4 \times A$ & 0.158 & 0.404 & 0.288 \\
48 & $4 \times A$ & 0.018 & 0.108 & 0.010 \\
54 & $4 \times A$ & 0.112 & 0.108 & 2.012 \\
75 & $4 \times A$ & 4.632 & 3.252 & 0.860 \\
17 & $4 \times A$ & 0.356 & 0.016 & 0.535 \\
79 & $4 \times A$ & 0.050 & 2.140 & 0.116 \\
83 & $4 \times A$ & 0.032 & 0.612 & 0.060 \\
205 & $4 \times A$ & 0.023 & 0.562 & 0.022 \\
104 & $4 \times A$ & 0.096 & 0.148 & 1.056 \\
114 & $4 \times A$ & 0.259 & 0.124 & 0.059 \\
111 & $4 \times A$ & 0.002 & 0.336 & 0.085 \\
81 & $4 \times A$ & 2.004 & 2.140 & 0.152 \\
\hline
\end{tabular}

A5. Candidate loci and environmental correlations. Non = nonmethylated, int = internally methylated, ext $=$ externally methylated, Temp. = temperature

\begin{tabular}{llllll}
\hline Marker & Environm. Var. & Loglikelihood & G Score & Wald Score & BIC \\
\hline non47 & Altitude & -57.493 & 38.676 & 27.245 & 134.235 \\
& Mean Annual Temp. & -49.710 & 54.242 & 32.306 & 118.669 \\
non129 & Altitude & -65.708 & 37.264 & 24.969 & 150.665 \\
& Mean Annual Temp. & -56.764 & 55.152 & 32.641 & 132.778 \\
non131 & Mean Annual Temp. & -56.839 & 47.873 & 31.013 & 132.927 \\
non157 & Mean Annual Temp. & -51.815 & 33.033 & 23.407 & 122.878 \\
non168 & Altitude & -55.070 & 50.276 & 30.350 & 129.389 \\
& Mean Annual Temp. & -44.356 & 71.704 & 35.403 & 107.961 \\
\multirow{2}{*}{ non171 } & Mean Annual Temp. & -64.642 & 32.267 & 24.342 & 148.533 \\
non173 & Altitude & -57.027 & 41.105 & 28.087 & 133.304 \\
& Mean Annual Temp. & -47.307 & 60.545 & 33.732 & 113.863 \\
\cline { 2 - 4 } non180 & Altitude & -62.894 & 33.423 & 24.666 & 145.036 \\
& Mean Annual Temp. & -54.399 & 50.412 & 31.727 & 128.046
\end{tabular}




\begin{tabular}{|c|c|c|c|c|c|}
\hline \multirow{2}{*}{ non250 } & Altitude & -46.971 & 32.941 & 24.647 & 113.190 \\
\hline & Mean Annual Temp. & -44.612 & 37.659 & 23.936 & 108.472 \\
\hline \multirow[t]{2}{*}{ ext17 } & Altitude & -55.971 & 41.720 & 28.377 & 131.191 \\
\hline & Mean Annual Temp. & -46.162 & 61.338 & 33.658 & 111.573 \\
\hline ext24 & Mean Annual Temp. & -48.821 & 34.316 & 23.471 & 116.890 \\
\hline \multirow[t]{2}{*}{ ext33 } & Altitude & -50.375 & 55.833 & 31.731 & 119.998 \\
\hline & Mean Annual Temp. & -42.905 & 70.772 & 34.984 & 105.060 \\
\hline \multirow[t]{2}{*}{ ext52 } & Altitude & -66.965 & 34.750 & 24.005 & 153.179 \\
\hline & Mean Annual Temp. & -62.764 & 43.152 & 29.102 & 144.777 \\
\hline ext56 & Mean Annual Temp. & -66.457 & 33.275 & 24.867 & 152.162 \\
\hline \multirow[t]{2}{*}{ ext74 } & Altitude & -55.339 & 41.413 & 28.331 & 129.926 \\
\hline & Mean Annual Temp. & $-45 \cdot 653$ & 60.783 & $33 \cdot 338$ & 110.555 \\
\hline ext76 & Mean Annual Temp. & -52.246 & 40.537 & 27.218 & 123.740 \\
\hline \multirow[t]{2}{*}{ ext86 } & Altitude & -55.145 & 51.261 & 30.437 & 129.538 \\
\hline & Mean Annual Temp. & $-43 \cdot 512$ & $74 \cdot 527$ & $35 \cdot 525$ & 106.273 \\
\hline \multirow[t]{2}{*}{ ext95 } & Altitude & $-53 \cdot 405$ & $49 \cdot 772$ & 30.557 & 126.059 \\
\hline & Mean Annual Temp. & -44.856 & 66.870 & $34 \cdot 715$ & 108.962 \\
\hline ext137 & Mean Annual Temp. & -49.271 & $33 \cdot 416$ & 23.118 & 117.790 \\
\hline \multirow[t]{2}{*}{ ext154 } & Altitude & -57.493 & $45 \cdot 430$ & 29.094 & 134.235 \\
\hline & Mean Annual Temp. & $-44 \cdot 554$ & 71.307 & $35 \cdot 385$ & 108.358 \\
\hline ext159 & Mean Annual Temp. & -48.665 & 37.026 & 24.752 & 116.578 \\
\hline ext186 & Mean Annual Temp. & -52.844 & 43.028 & 28.485 & 124.936 \\
\hline \multirow[t]{2}{*}{ ext197 } & Altitude & -52.083 & 36.847 & 26.700 & 123.415 \\
\hline & Mean Annual Temp. & -46.092 & 48.829 & 29.175 & 111.434 \\
\hline \multirow[t]{2}{*}{ ext199 } & Altitude & -65.634 & 35.643 & 24.769 & 150.517 \\
\hline & Mean Annual Temp. & -58.851 & 49.209 & 31.336 & 136.951 \\
\hline \multirow[t]{2}{*}{ ext210 } & Altitude & -45.748 & $35 \cdot 387$ & 25.682 & 110.744 \\
\hline & Mean Annual Temp. & -42.494 & 41.895 & 24.998 & 104.237 \\
\hline $\operatorname{ext} 216$ & Mean Annual Temp. & $-53 \cdot 510$ & 39.891 & 27.152 & 126.269 \\
\hline ext227 & Mean Annual Temp. & $-49 \cdot 330$ & 35.694 & 24.262 & $117 \cdot 910$ \\
\hline ext235 & Altitude & $-65 \cdot 402$ & $35 \cdot 384$ & 24.791 & 150.053 \\
\hline
\end{tabular}




\begin{tabular}{llllll} 
& Mean Annual Temp. & -56.363 & 53.462 & 32.581 & 131.974 \\
ext241 & Altitude & -55.319 & 36.274 & 26.438 & 129.887 \\
& Mean Annual Temp. & -47.142 & 52.627 & 31.015 & 113.534 \\
ext248 & Altitude & -55.858 & 31.345 & 24.159 & 130.965 \\
& Mean Annual Temp. & -49.344 & 44.373 & 28.291 & 117.938 \\
ext351 & Altitude & -54.694 & 56.009 & 30.682 & 128.637 \\
& Mean Annual Temp. & -40.373 & 84.652 & 34.728 & 99.994 \\
ext358 & Altitude & -59.037 & 34.016 & 25.298 & 137.323 \\
& Mean Annual Temp. & -52.835 & 46.420 & 29.958 & 124.919 \\
int32 & Mean Annual Temp. & -56.806 & 55.526 & 30.159 & 132.861 \\
int92 & Altitude & -67.984 & 34.538 & 22.872 & 155.216 \\
& Mean Annual Temp. & -60.708 & 49.091 & 30.271 & 140.664 \\
int228 & Mean Annual Temp. & -60.558 & 40.436 & 25.154 & 140.364 \\
int290 & Mean Annual Temp. & -64.360 & 34.891 & 23.684 & 147.970 \\
\hline
\end{tabular}




\section{Discussion}

In this thesis, I dealt various issues of plant evolution related to polyploidy, apomixis as well as Geographical Parthenogenesis. The studied plant species Ranunculus kuepferi Greuter \& Burdet resembles an ideal model system consisting of different cytotypes that expresses diverging distribution ranges. Diploids are mainly restricted to the Southwestern Alps, while tetraploids are widespread over the whole central to Eastern Alps (Cosendai and Hörandl 2010). Other cytotypes are rare and occur only in the sympatric contact zone between both dominating ploidy levels. Although I would like to emphasize at this point that this work has not answered all research questions nor in any way claims to be complete, the herein presented results are able to expand the understanding of plant evolution and shed some light on specific issues that are linked to the variation and distribution of reproductive modes among different cytotypes, environmental influences, as well as the genetic and epigenetic background.

\section{Cytotype composition, reproductive modes and fitness}

Flow Cytometric Seed Screening (FCSS, Chapter 1) was used to assess variance and geographical patterns of the reproduction mode among different cytotypes and populations of 551 R. kuepferi individuals from 81 sampling sites, presenting the first quantitative and comprehensive analysis of seed formation in this model species. Most literature neglect the process of disturbed female gametogenesis (Mirzaghaderi and Hörandl 2016), although it is a prerequisite for apomixis and maybe polyploidization.

Results revealed a high diversity in the reproductive pathways on individual as well as population level. In the tetraploid cytotype facultative to obligate apomixis is the predominant mode of reproduction. Although facultative sexuality is quite common for aposporous plants, as apomictic and sexual meiotic development initially run in parallel (Asker and Jerling 1992, Krahulcová et al. 2004, Dobeš et al. 2013, Klatt et al. 2016), the observed high degree of sexual seed formation among tetraploids is remarkably high. The previously common assumption that apomixis and polyploidy are almost exclusively correlated (Gustafsson 1946, Bierzychudek 1985, Carman 1997, Koltunow and Grossniklaus 2003) is contradicted by our results. We found a small portion of six individuals from three different diploid populations that expressed a mixed mode of reproduction with some apomictically formed seeds. Apomixis in diploids is so far only known from diploid hybrids of the genus Boechera (Dobeš et al. 
2006, Aliyu et al. 2010), as well as in Paspalum (Siena et al. 2008). Geographic isolation of those populations suggests multiple emergence of apomixis in unrelated individuals and rules out introgression of neighboring tetraploids as apparent explanation (Hörandl and Temsch 2009). Due to the low occurrence of apomictic seed production in natural diploids of $R$. kuepferi, large scale seed screenings are needed to detect such rare events.

Ramsey and Schemske (1998) suggested temperature shocks as trigger for a switch to apomixis, which would apply so for cold stress. The mixed reproducing diploid individuals in fact originate from populations that on average occur at higher elevations. However, this cannot be accurately associated with any possible cold shocks, due to the fact that the BIOCLIM dataset we used to assess the influence of environmental factors covers only a $1 \mathrm{~km} \times 1 \mathrm{~km}$ grid at minimum and is averaged over 30 years (Hijmans et al. 2005). Neither microclimatic effects nor influences of climatic fluctuations of the sampling years can therefore be included.

On the other hand, the initial hypothesis that cold stress may trigger apomixis is supported by experimental work with the same R. kuepferi plants in climate chambers. Klatt et al. (2018) found formerly obligate sexual diploids spontaneously producing apomictic seeds $(4.4 \%)$ in cold treatments, while in tetraploids facultative sexuality was reduced to nearly zero. Temperature was also the most decisive environmental factor between cytotypes in in an ecological study of Kirchheimer et al. (2016). Strikingly, cold treatments directly caused fully functional apomixis, where apomeiosis is immediately coupled to parthenogenesis. Only a tiny fraction (1.1\%) of seeds among tetraploids were formed by partial apomixis ( $B_{\text {III }}$ hybrids). These findings are comparable with results of spontaneous apospory in diploid hybrids of Ranunculus auricomus (Hojsgaard et al. 2014). But there is no plausible indication of a hybrid origin in R. kuepferi, as they are assumed to be of autopolyploid origin (Burnier et al. 2009, Cosendai et al. 2011, 2013). Hence, hybridity as well as polyploidy cannot be the sole causes of unreduced egg cell formation in $R$. kuepferi.

Seed set is significantly lower in tetraploids, which is maybe caused by difficulties in chromosome segregation due to multivalent formation at meiosis in autotetraploids (Cosendai et al. 2011, Cohen et al. 2013, Lloyd and Bomblies 2016). Seed set among diploids shows strong negative correlation to altitude, which is maybe influenced by pollinator limitation and a generally worse adaptation of their reproductive tissues to cold temperatures (e.g. Körner 2003, Körner 2006, Orsenigo et al. 2014). 


\section{Geographical patterns and environmental influences}

Although overall reproductive fitness in tetraploid apomicts is worse compared to diploids, they represent the most successful cytotype referring to their observed range expansion (Cosendai and Hörandl 2010, Cosendai et al. 2013, Schinkel et al. 2016). Possibly, this is linked to advantages of polyploidy itself and whole genome doubling (WGD) in particular (Ohno 1970, Comai 2005, Otto 2007, Soltis and Soltis 2009). Performance of polyploids is often increased due to the heterosis effect (Birchler et al. 2010), which is even more pronounced in autopolyploids (Bingham et al. 1994, Birchler et al. 2010). Contrary to expectations regarding heterosis, tetraploids of $R$. kuepferi exhibit a generally smaller growth. Low temperatures at high altitudes shorten the growing season and slow down cell metabolism and differentiation (Stitt and Hurry 2002, Körner 2006, 2016). Alpine plants mostly produce fewer but larger cells with a beneficial surface to volume ratio as adaptation to the harsh environment, which is known as alpine dwarfism (Körner 2003, 2016). This serves as avoidance of frost damage when plants and especially their high metabolic reproductive tissues are exposed and not covered by snow, especially during the growth period (Ladinig et al. 2013). Further advantages of polyploidy affect both the carbon intake ability under lower $\mathrm{CO}_{2}$ partial pressure on high altitudes, and the electron transport capability under high UV radiation (Körner 2003).

Higher rates of heterozygosity, helping polyploids to prevent detrimental effects of inbreeding, especially when genetically bottlenecked, like in small founder populations (Comai 2005). Autopolyploids maintain heterozygosity even better and WGD further provides the ability for diversification of gene functions (Prince and Pickett 2002, Adams and Wendel 2005, Moore and Purugganan 2005). It is also known that sub- and neofunctionalization of redundant genes are related to niche shifts (Lynch and Walsh 2007). However, recent studies suggest that formation and persistence of polyploid complexes do not necessarily depend on either shifts of niche optima or a broadening of the niche (Theodoridis et al. 2013, Glennon et al. 2014, Kirchheimer et al. 2016, 2018). Calculated niche divergence in the sympatric and allopatric range of diploid and polyploid cytotypes of $R$. kuepferi revealed that tetraploid populations showed a significant niche shift in the allopatric range towards colder temperatures, higher precipitation and slightly more acid soils, while niche breadth decreased compared to diploids (Kirchheimer et al. 2016). In the sympatric area, tetraploids were neither able to establish in the warmer niche range of diploids, nor to ingress it subsequently (Kirchheimer et 
al. 2016). Avoidance of Minority Cytotype Exclusion (MCE) might be more important for polyploid individuals than differentiation of abiotic niches to withstand larger densities of diploids (Levin 1975, Baack 2005). Hence, the observed niche differentiation in tetraploid $R$. kuepferi probably developed during or even after their geographical range expansion, indicating that it is not a prerequisite of tetraploid establishment and the change in the reproductive system might be more influential (Kirchheimer et al. 2016). In contrast, simulations of range dynamics with assessment of postglacial migration rates suggest a dominant effect of niche differentiation on the current distribution characterized by Geographical Parthenogenesis, despite niche restriction in tetraploids (Kirchheimer et al. 2018). A switch to asexual reproduction and polyploidization may induce a higher tolerance of cooler and more extreme conditions, which in turn provides the ability to overcome high elevation barriers and migrate into higher altitudes (Kirchheimer et al. 2018). Priority effects then promote an additional competitive advantage and act inhibitory on the progression of sexual diploids (Kirchheimer et al. 2018). However, these considerations remain largely speculative. Hörandl (2006) concluded that the causalities for distributional advantage of asexuality are probably complex and that determinants may interact with each other and with the abiotic as well as biotic environment. Differences in performance of diploid and tetraploid cytotypes of $R$. kuepferi in direct competition with the surrounding vegetation were not significant. $R$. kuepferi is one of the first plants to sprout after snow melting and exhibit a very fast growth and development phase until it is ready for fruiting (personal observations). Neighboring plant species develop significantly slower and therefore hardly compete for resources in the early vegetation period. Biotic interactions with other plants seem to play a minor role for the geographical patterns (Kirchheimer et al. 2016).

According to Baker's Law, uniparental reproduction provides the substantial advantage that a single propagule is theoretically sufficient for founding a new population (Stebbins 1950, Baker 1967). Especially after long-distance dispersal events Allee effects would otherwise slow down expansion rates of sexually reproducing populations, due to limitation of mating partners (Courchamp et al. 2008). Most apomictic plant species are self-fertile avoiding the need for mates and pollinators but originate from self-incompatible outcrossing diploids (Hörandl 2010). Self-fertility combined with an accelerated seed production due to apomixis would make Baker's Law a reasonable cause for the occurrence of Geographical Parthenogenesis. 
On the other hand, no apparent correlation of reproduction mode to geographical patterns was found in tetraploid populations of $R$. kuepferi. The degree of facultative sexuality is scattered across tetraploid populations over the whole distribution area in the Alps. Additionally, even apomictic tetraploids lack a distinct population genetic structure as shown before (Cosendai et al. 2013), which supports the premises of the FNV model and consequently rejects the GPG model of a widespread general genotype. But the lack of clonality opposes the idea of particular ecological niches stabilized by different clones. Genetic diversity within and between populations occurs due to the relatively high degree of facultative sexuality that causes allelic mixing of regional gene pools, as it is further supported by Kirchheimer et al. (2018). Prevalence of long-distance dispersal as founder event of apomictic populations and sole reason for their establishment seems to be unlikely. Rather a stepwise dispersal promoted by self-fertility and apomixis can be considered as explanation for the apparent migration success.

However, apomictic tetraploids were on average found at higher elevations indicating a positive correlation of apomictic reproduction with altitude and related climatic factors (cold temperatures), although the mode of reproduction cannot be entirely separated from ploidy effects. The observed niche shift to colder temperatures (Kirchheimer et al. 2016, 2018) is therefore most likely an effect of elevation, not latitude. Otherwise northernmost populations should occur at lower altitudes, as they would try to keep their niche constant (Körner 2003). This is not the case in $R$. kuepferi and in accordance with the general trend of apomictic plants to occupy more extreme and marginal habitats (Bierzychudek 1985, Hörandl 2006, Vrijenhoek and Parker 2009).

\section{Origins of polyploidy and apomixis}

Based on the comprehensive FCSS dataset, we focused on the previously neglected triploid cytotype and the few but all the more interesting cases of $B_{\text {III }}$ hybrids, that were found in among all cytotypes. $B_{\text {III }}$ hybrids are neopolyploids formed via unreduced egg cells that are fertilized, as a pathway of polyploidization based on ploidy shifts in the seed (Comai 2005, Hörandl and Hojsgaard 2012). We examined seeds with a changed ploidy compared to their mother plants regarding possible pathways of their emergence.

One of our key findings is the absence of paternal triploidization. We found no hints for a male triploid bridge, due to the absence of triploid seeds resulting from $2 \mathrm{n}$ pollen. The origin 
of triploids by backcrossing of diploid mother plants with unreduced tetraploid pollen can therefore be considered as highly unlikely. Seeds with ploidy shifts in the embryo predominantly consisted of unreduced female gametes with various different male contributions. Tetraploid individuals exhibit no obligate sexual reproduction as it would be expected in a male-dependent sexual polyploidization process by pollen. As apospory is supposed to be a heritable trait (Nogler 1984, Ozias-Akins and Van Dijk 2007), tetraploids should inherit this reproductive trait from the female variant of unilateral sexual polyploidization. Indeed, there was evidence found that $2 \mathrm{n}$ pollen caused biparental tetraploidization in some examples, but this could also be the result of aneuploid pollen of recently established triploids. Absence of triploid seeds resulting from $2 \mathrm{n}$ pollen and predominant obligate apomixis among tetraploids suggest that polyploidization in $R$. kuepferi occurs through a two-step pathway of unilateral female sexual tetraploidization, that we call female triploid bridge. The probability for direct one-step tetraploidization via unreduced female and male gametes is meanwhile lower. Anyway, shifts in ploidy and reproduction mode to asexual seed formation seems to occur in diploids at first. This represents a novel aspect in the evolution of apomixis.

Previous studies on triploids assumed their fertility to be low (Ramsey and Schemske 1998, Hörandl and Temsch 2009). However, more recent studies suggest that triploids are able to produce euploid gametes to some degree and are therefore semi-fertile (Ramsey and Schemske 1998). Although the reproductive fitness is lower in triploids of $R$. kuepferi, the observed incidence of many mature seeds affirms that assumption. Most analyzed triploid seeds are of apomictic origin, which is a common strategy of polyploids to prevent $\mathrm{F}_{1}$-sterility (e.g. Hojsgaard et al. 2014) and could help to prevent complications, which would occur during meiotic segregation. Another difficulty in interploidy crosses affects the endosperm balance in seeds (Scott 2007, Köhler et al. 2010). Deviations from the optimal two maternal to one paternal genome contribution of the endosperm and associated aberrations in gene expression often cause seed abortion, especially when paternal genome excess occurs (Haig and Westoby 1991, Vinkenoog et al. 2003, Köhler et al. 2010). In R. kuepferi only triploid seeds were found that are formed by unreduced female gametes fertilized by a reduced pollen. The accompanied endosperm is then in fact unbalanced (4m:1p), but $R$. kuepferi seems to tolerate such lower paternal genome contributions better than paternal excess and forms viable seeds. This is further supported by the identification of some cases of autonomous endosperm development (Cosendai and Hörandl 2010, Schinkel et al. 2016). 
According to Ramsey and Schemske (1998), the observed number of well-developed seeds among triploids of $R$. kuepferi seems to be sufficient as factor for establishment of triploid groups, that would allow for further polyploidization steps. By fertilization of unreduced female gametes, triploid $B_{\| I}$ hybrids are easily generated in sufficient numbers to serve as triploid bridge, producing triploid offspring or again $B_{\text {III }}$ hybrids of higher ploidy.

\section{Determination of pollen ploidy}

Some specific endosperm to embryo ratios in our FCSS data indicate the possibility that unreduced pollen might be involved in the genesis of several asexually formed tetraploid seeds. On the other hand, it is impossible to distinguish between contribution of either one unreduced or two reduced sperm nuclei, as it is also conceivable (Talent and Dickinson 2007, Šarhanová et al. 2012, Ludwig et al. 2013, Burgess et al. 2014). In many plant taxa, pollen ploidy can be directly inferred from pollen size (Ramsey and Schemske 1998), as 2n pollen grains are usually 30-40\% larger and distribution between reduced and unreduced pollen is mostly bimodal (Róis et al. 2012, Cohen et al. 2013, De Storme et al. 2013; Marinho et al. 2014).

A high-throughput method for volumetric pollen size determination (De Storme et al. 2013) revealed a generally high variation in pollen size for both cytotypes in $R$. kuepferi, forming four distinctive size classes. We found tetraploid pollen to be generally larger than in diploids, which is in accordance with Huber (1988). Pollen grains in the very small as well as very large size classes was mostly malformed. Microscopic viability staining confirmed a high amount of aborted pollen grains, which is typical for facultative apomictic plants (Izmailow 1996, Hörandl et al. 1997, Voigt et al. 2007). This most likely results from developmental disturbances during microgametogenesis (Hojsgaard et al. 2014). Since the sizes between diploid and tetraploid pollen overlap significantly, it is impossible to estimate the ploidy solely on the basis of size.

\section{Epigenetic patterns dependent on cytotype and reproduction mode}

As mentioned before, tetraploids have high levels of individual genetic diversity and exhibit a lack of private alleles (AFLP) that suggests low genetic differentiation. Diploids express isolation-by-distance profiles, but no such geographical structure among tetraploids is found. Changes in growth (tetraploid dwarfism), reproduction (apomixis) and niche shifts (towards colder climates and higher altitudes) must be rather due to epigenetic modifications. 
We explored epigenetic patterns in regard with preassigned differences in the respective reproduction modes among both the di- and tetraploid cytotype (Chapter 3 ). In plants epigenetic mechanisms such as methylation seem to be highly pronounced and promote gene regulation (e.g. Pikaard and Scheid 2014). In R. kuepferi, a significant differentiation was observed between di- and tetraploids. Additionally, tetraploids showed a striking difference between apomictic and mixed reproduction, while such divergence could not be determined among diploids, but maybe caused by the limited sampling in mixed reproducing individuals. Methylation patterns exhibit a random distribution with no significant spatial auto-correlation among di- and tetraploid populations on global scale. On the other hand, correlations to altitude and adjunctive environmental factors (temperature, precipitation) was found, though mostly reflects regional distribution patterns and linked climatic niches. In diploids this is maybe caused by a generally warmer and dryer niche, physical proximity of populations and a smaller altitudinal range. Rapid colonization and a high degree of facultative sexuality could have facilitated the unstructured patterns among tetraploids.

In accordance with the findings of Cosendai et al. (2013) we found differentiation between diand tetraploids in the methylated, but not in the unmethylated epiloci. Interestingly, mixed reproducing diploids seem to be more similar to mixed reproducing tetraploids. These diploids may have an epigenetic background for transition, as we hypothesized that tetraploids formed by a female triploid bridge from facultative apomictic diploid individuals. Preservation of facultative sexuality may demand similar methylation patterns that represent similar genetic regulation networks for the meiotic pathway, while in contrast asexual reproduction is possibly connected to demethylation and overexpression of regulating gene networks which are under epigenetic control, leading to aberrations in the precisely coordinated meiotic process (Laspina et al. 2008, Polegri et al. 2010, Feletti et al. 2011, Podio et al. 2014, Rodrigo et al. 2017). Resulting sterility could then be circumvented by apomictic reproduction and coherent methylation patterns would be transmitted to subsequent generations.

In this regard, relative contributions of $\mathrm{CG}$ or $\mathrm{CHG}$ and $\mathrm{CHH}$ sequence methylation could be important (Gruenbaum et al. 1981, Belanger and Hepburn 1990, Kovarik et al. 1997, Alonso et al. 2016, Gouil and Baulcombe 2016). Higher variation of internally holomethylated epiloci (CG context) among tetraploids represent heritable and more conserved methylation patterns, while external methylation (CHG context) is less stable and only partly heritable 
(Becker et al. 2011, Paszkowski and Grossniklaus 2011, Takuno and Gaut 2013). Ploidy alterations up to polyploidy being related to the different expression of CG and CHG methylation, which can have important influence on transposable elements, as well as genetic expression and inheritance (Zhang et al. 2015; Gao et al. 2016; Baidouri et al. 2018). Therefore, polyploidization maybe increased physiological tolerance to withstand harsher environmental conditions like colder climates, due to altered gene expression patterns by doubled alleles (Wang et al. 2004, Hegarty et al. 2006, 2011). Reproduction in R. kuepferi with regard to different environmental influences is indeed variable, expressing phenotypic plasticity in same individuals over a single flowering season (Klatt et al. 2018).

Although methylation patterns were investigated in leave material, our findings support the hypothesis that epigenetic response to physiological stress in the whole plant induces global DNA demethylation and hence influences the mode of reproduction (Hörandl and Hadacek 2013, Klatt et al. 2016), as seen in other examples e.g. Boechera (Shah et al. 2016) or Eragrostis curvula (Rodrigo et al. 2017). Indeed, obligate tetraploid apomicts of $R$. kuepferi exhibit a lower amount of epiloci than diploids and might represent a lineage that experienced cold stress resulting in demethylation and the establishment of apomixis, that in turn preserves methylation patterns over generations, as meiotic recombination is bypassed. 


\section{References}

Adams KL and Wendel JF. 2005. Polyploidy and genome evolution in plants. Current Opinion in Plant Biology 8:135-141

Aliyu OM, Schranz ME and Sharbel TF. 2010. Quantitative variation for apomictic reproduction in the genus Boechera (Brassicaceae). American Journal of Botany 97:1719-1731

Alonso C, Pérez R, Bazaga P, Medrano M and Herrera CM. 2016. MSAP markers and global cytosine methylation in plants: a literature survey and comparative analysis for a wild-growing species. Molecular Ecology Resources 16:80-90

Asker SE and Jerling L. 1992. Apomixis in Plants. CRC Press, Boca Raton

Baack EJ. 2005. Ecological factors influencing tetraploid, establishment in snow buttercups (Ranunculus adoneus, Ranunculaceae): Minority cytotype exclusion and barriers to triploid formation. American Journal of Botany 92:1827-1835

Baidouri ME, Kim KD, Abernathy B, Li Y-H, Qiu L-J and Jackson SA. 2018. Genic C-Methylation in Soybean Is Associated with Gene Paralogs Relocated to Transposable Element-Rich Pericentromeres. Molecular Plant 11(3):485-495

Baker HG. 1967. Support for Baker's Law-as a rule. Evolution 21:853-856

Becker C, Hagmann J, Müller J, Koenig D, Stegle O, Bogwardt K and Weigel D. 2011. Spontaneous epigenetic variation in the Arabidopsis thaliana methylome. Nature 480:245-250

Belanger FC and Hepburn AG. 1990. The evolution of CpNpG methylation in plants. Molecular Ecology 30:26-35

Bierzychudek P. 1985. Patterns in plant parthenogenesis. Experientia 41:1255-1264

Bingham ET, Groose RW, Woodfield DR and Kidwell KK. 1994. Complementary gene interactions in alfalfa are greater in autotetraploids than diploids. Crop Science 34:823-829

Birchler JA, Yao H, Chudalayandi S, Vaiman D and Veitia RA. 2010. Heterosis. The Plant Cell 22:21052112

Burgess MB, Cushman KR, Doucette ET, Talent N, Frye CT and Campbell CS. 2014. Effects of apomixis and polyploidy on diversification and geographic distribution in Amelanchier (Rosaceae). American Journal of Botany 101:1375-1387

Burnier J, Buerki S, Arrigo N, Küpfer P and Alvarez N. 2009. Genetic structure and evolution of Alpine polyploid complexes: Ranunculus kuepferi (Ranunculaceae) as a case study. Molecular Ecology 18:3730-3744

Carman JG. 1997. Asynchronous expression of duplicate genes in angiosperms may cause apomixis, bispory, tetraspory, and polyembryony. Biological Journal of the Linnean Society 61:51-94

Cohen H, Fait A and Tel-Zur N. 2013. Morphological, cytological and metabolic consequences of autopolyploidization in Hylocereus (Cactaceae) species. BioMed Central Plant Biology 13:173-186

Comai L. 2005. The advantages and disadvantages of being polyploid. Nature Reviews Genetics 6:836-846 
Cosendai A-C and Hörandl E. 2010. Cytotype stability, facultative apomixis and Geographical Parthenogenesis in Ranunculus kuepferi (Ranunculaceae). Annals of Botany 105:457-470

Cosendai A-C, Rodewald J and Hörandl E. 2011. Origin and distribution of autopolyploids via apomixis in the alpine species Ranunculus kuepferi (Ranunculaceae). Taxon 60:355-364

Cosendai A-C, Wagner J, Ladinig U, Rosche C and Hörandl E. 2013. Geographical parthenogenesis and population genetic structure in the alpine species Ranunculus kuepferi (Ranunculaceae). Heredity 110:560-569

Courchamp F, Berec J and Gascoigne J. 2008. Allee effects in ecology and conservation. Oxford University Press, Oxford

De Storme N, Zamariola L, Mau M, Sharbel TF and Geelen D. 2013. Volume-based pollen size analysis: an advanced method to assess somatic and gametophytic ploidy in flowering plants. Plant Reproduction 26:65-81

Dobeš C, Koch M and Sharbel TF. 2006. Embryology, karyology, and modes of reproduction in the north american genus Boechera (Brassicaceae): a compilation of seven decades of research. Annals of the Missouri Botanical Garden 93:517-534

Dobeš C, Lückl A, Hülber K and Paule J. 2013. Prospects and limits of the flow cytometric seed screen insights from Potentilla sensu lato (Potentilleae, Rosaceae). New Phytologist 198:605-616

Felitti SA, Seijo JG, González AM, Podio M, Laspina NV, Siena L, Ortiz JPA and Pessino SC. 2011. Expression of LORELEI-like genes in aposporous and sexual Paspalum notatum plants. Plant Molecular Biology 77:337-354

Gao L, Diarso M, Zhang A, Zhang H, Dong Y, Liu L, Lv Z and Liu B. 2016. Heritable alteration of DNA methylation induced by whole-chromosome aneuploidy in wheat. New Phytologist 209:364-375

Gouil Q and Baulcombe DC. 2016. DNA Methylation Signatures of the Plant Chrmomethyltransferases. PLOS Genetics 12(12):e1006526

Gruenbaum Y, Navehmany T, Cedar H and Razin A. 1981. Sequence specificity of methylation in higher plant DNA. Nature 292:860-862

Gustafsson A. 1946. Apomixis in higher plants. Lunds Universitets Årsskrift NF /I 42(3):1-67

Haig D and Westoby M. 1991. Genomic imprinting in the endosperm: its effect on seed development in crosses between species, and between different ploidies of the same species, and its implications for the evolution of apomixis. Philosophical Transactions of The Royal Society B: Biological Sciences 333:1-13

Hegarty MJ, Barker GL, Wilson ID, Abbott RJ, Edwards KJ and Hiscock SJ. 2006. Transcriptome shock after interspecific hybridization in Senecio is ameliorated by genome duplication. Current Biology $16: 1652-1659$

Hegarty MJ, Batstone T, Barker GL, Edwards KJ, Abbott RJ and Hiscock SJ. 2011. Nonadditive changes to cytosine methylation as a consequence of hybridization and genome duplication in Senecio (Asteraceae). Molecular Ecology 20:105-113 
Hijmans RJ, Cameron SE, Parra JL, Jones PG and Jarvis A. 2005. Very high resolution interpolated climate surfaces for global land areas. International Journal of Climatology 25:1965-1978

Hojsgaard D, Greilhuber J, Pellino M, Paun O, Sharbel TF and Hörandl E. 2014. Emergence of apospory and bypass of meiosis via apomixis after sexual hybridisation and polyploidisation. New Phytologist 204:1000-1012

Hörandl E. 2006. The complex causality of Geographical Parthenogenesis. New Phytologist 171(3):525-538

Hörandl E. 2010. The evolution of self-fertility in apomictic plants. Sexual Plant Reproduction 23:73-86

Hörandl E and Hadacek F. 2013. The oxidative damage initiation hypothesis of meiosis. Plant Reproduction $26: 351-367$

Hörandl E and Hojsgaard D. 2012. The evolution of apomixis in angiosperms: a reappraisal. Plant Biosystems 146(3):681-693

Hörandl E and Temsch EM. 2009. Introgression of apomixis into sexual species is inhibited by mentor effects and ploidy barriers in the Ranunculus auricomus complex. Annals of Botany 104:81-89

Hörandl E, Dobeš CH and Lambrou M. 1997. Chromosomen- und Pollenuntersuchungen an österreichischen Sippen des Ranunculus auricomus-Komplexes. Botanica Helvetica 107:195-209

Huber W. 1988. Natürliche Bastardierungen zwischen weissblühenden Ranunculus-Arten in den Alpen [Natural hybridizations between white-flowered species of Ranunculus in the Alps]. Veröffentlichungen des Geobotanischen Institutes der Eidgenössischen Technischen Hochschule, Stiftung Rübel, Zürich 100:1-160

Izmailow R. 1996. Reproductive strategy in the Ranunculus auricomus complex (Ranunculaceae). Acta Societatis Botanicorum Poloniae 65:167-170

Kirchheimer B, Schinkel CCF, Dellinger AS, Klatt S, Moser D, Winkler M, Lenoir J, Caccianiga M, Guisan A, Nieto-Lugilde D, Svenning J-C, Thuiller W, Vittoz P, Willner W, Zimmermann NE, Hörandl E and Dullinger S. 2016. A matter of scale: apparent niche differentiation of diploid and tetraploid plants may depend on extent and grain of analysis. Journal of Biogeography 43:716-726

Kirchheimer B, Wessely J, Gattringer A, Hülber K, Moser D, Schinkel CCF, Appelhans M, Klatt S, Caccianiga M, Dellinger A, Guisan A, Kuttner M, Lenoir J, Maiorano L, Nieto-Lugilde D, Plutzar C, Svenning J-C, Willner W, Hörandl E and Dullinger S. 2018. Reconstructing Geographical Parthenogenesis: effects of niche differentiation and reproductive mode on Holocene range expansion of an alpine plant. Ecology Letters 21:392-401

Klatt S, Hadacek F, Hodač L, Brinkmann G, Eilerts M, Hojsgaard D and Hörandl E. 2016. Photoperiod extension enhances sexual megaspore formation and triggers metabolic reprogramming in facultative apomictic Ranunculus auricomus. Frontiers in Plant Science 7:278-293

Klatt S, Schinkel CCF, Kirchheimer B, Dullinger S and Hörandl E. 2018. Effects of cold treatments on fitness and mode of reproduction in the diploid and polyploid alpine plant Ranunculus kuepferi (Ranunculaceae). Annals of Botany 121(8):1287-1298 
Köhler C, Mittelsten-Scheid O and Erilova A. 2010. The impact of the triploid block on the origin and evolution of polyploid plants. Trends in Genetics 26:142-148

Koltunow AM and Grossniklaus U. 2003. Apomixis: a developmental perspective. Annual Review of Plant Biology 54:547-574

Körner C. 2003. Alpine plant life: functional plant ecology of high mountain ecosystems; with 47 tables. Springer, Berlin

Körner C. 2006. Significance of Temperature in Plant Life. In: Morison Jl, Morecroft MD (eds) Plant Growth and Climate Change. Blackwell Publishing Ltd., Oxford, pp 48-69

Körner C. 2016. Plant adaptation to cold climates. F10ooResearch 5(F1000 Faculty Rev):2769

Kovařik A, Matyášek R, Leitch A, Gazdová B, Fulneček J and Bezděk M. 1997. Variability in CpNpG methylation in higher plant genomes. Gene 204:25-33

Krahulcová A, Papouskuvá S and Krahulec F. 2004. Reproduction mode in the allopolyploid facultatively apomictic hawkweed Hieracium rubrum (Asteraceae, H. subgen. Pilosella). Hereditas 141:19-30

Ladinig U, Hacker J, Neuner G and Wagner J. 2013. How endangered is sexual reproduction of highmountain plants by summer frosts? Frost resistance, frequency of frost events and risk assessment. Oecologia 171:743-76o

Laspina NV, Vega T, Seijo JG, González AM, Martelotto LG, Stein J, Podio M, Ortiz JPA, Echenique VC, Quarin CL and Pessino SC. 2008. Gene expression analysis at the onset of aposporous apomixis in Paspalum notatum. Plant Molecular Biology 67:615-628

Levin DA. 1975. Minority cytotype exclusion in local plant populations. Taxon 24:35-43

Lloyd A and Bomblies K. 2016. Meiosis in autopolyploid and allopolyploid Arabidopsis. Current Opinion in Plant Biology 30:116-122

Ludwig S, Robertson A, Rich TCG, Djordjevic M, Cerovic R, Houston L, Harris SA and Hiscock SJ. 2013. Breeding systems, hybridization and continuing evolution in Avon Gorge Sorbus. Annals of Botany 111:563-575

Lynch M and Walsh B. 2007. The origins of genome architecture. Sinaver Associates, Sunderland

Marinho RC, Mendes-Rodrigues C, Bonetti AM and Oliveira PE. 2014. Pollen and stomata morphometrics and polyploidy in Eriotheca (Malvaceae-Bombacoideae). BMC Plant Biology 16:508-511

Mirzaghaderi G, HörandI E. 2016. The evolution of meiotic sex and its alternatives. Proceedings of the Royal Society B: Biological Sciences 283:20161221

Moore RC and Purugganan MD. 2005. The evolutionary dynamics of plant duplicate genes. Current Opinion in Plant Biology 8(2):122-128

Nogler GA. 1984. Gametophytic apomixis. In: Johri PBM (eds) Embryology of angiosperms. Springer, Berlin, $p p$ 475-518

Ohno S. 1970. Evolution by gene duplication. Springer, Berlin

Orsenigo S, Mondoni A and Rossi G. 2014. Some like it hot and some like it cold, but not too much: plant responses to climate extremes. Plant Ecology 215(7):677-688 
Otto SP. 2007. The evolutionary consequences of polyploidy. Cell 131:452-462

Ozias-Akins P and Van Dijk PJ. 2007. Mendelian genetics of apomixis in plants. Annual Review of Genetics 41:509-537

Paszkowski J and Grossniklaus U. 2011. Selected aspects of transgenerational epigenetic inheritance and resetting in plants. Current Opinion in Plant Biology 14(2):195-203

Pikaard CS and Scheid OM. 2014. Epigenetic Regulation in Plants. Cold Spring Harbor Perspectives Biology 6(12):a019315

Podio M, Felitti SA, Siena LA, Delgado L, Mancini M, Seijo JG, González AM, Pessino SC and Ortiz JPA. 2014. Characterization and expression analysis of SOMATIC EMBRYOGENESIS RECEPTOR KINASE (SERK) genes in sexual and apomictic Paspalum notatum. Plant Molecular Biology 84:479495

Polegri L, Calderini O, Arcioni S and Pupilli F. 2010. Specific expression of apomixis-linked alleles revealed by comparative transcriptomic analysis of sexual and apomictic Paspalum simplex Morong flowers. Journal of Experimental Botany 61(6):1869-1883

Prince VE and Pickett FB. 2002. Splitting pairs: the diverging fates of duplicated genes. Nature Reviews Genetics 3:827-837

Ramsey J and Schemske DW. 1998. Pathways, mechanisms, and rates of polyploid formation in flowering plants. Annual Review of Ecology and Systematics 29:467-501

Rodrigo JM, Zappacosta DC, Selva JP, Garbus I, Albertini E and Echenique V. 2017. Apomixis frequency under stress conditions in weeping lovegrass (Eragrostis curvula). PLOS ONE 12(4):e0175852

Róis AS, Teixeira G, Sharbel TF, Fuchs J, Martins S, Espírito-Santo D and Caperta AD. 2012. Male fertility versus sterility, cytotype, and DNA quantitative variation in seed production in diploid and tetraploid sea lavenders (Limonium sp., Plumbaginaceae) reveal diversity in reproduction modes. Sexual Plant Reproduction 25:305-318

Šarhanová $P$, Vašut RJ, Dančák $M$, Bureš $P$ and Trávníček B. 2012. New insights into the variability of reproduction modes in European populations of Rubus subgen. Rubus: how sexual are polyploid brambles?. Sexual Plant Reproduction 25:319-335

Schinkel CCF, Kirchheimer B, Dellinger AS, Klatt S, Winkler M, Dullinger S and Hörandl E. 2016. Correlations of polyploidy and apomixis with elevation and associated environmental gradients in an alpine plant. AoB PLANTS 8:plwo64

Scott RJ. 2007. Polyspermy in apomictic Crataegus: yes and no. New Phytologist 173:227-230

Shah JN, Kirioukhova O, Pawar P, Tayyab M, Mateo JL and Johnston AJ. 2016. Depletion of Key Meiotic Genes and Transcriptome-Wide Abiotic Stress Reprogramming Mark Early Preparatory Events Ahead of Apomeiotic Transition. Frontiers in Plant Science 7:1539

Siena LA, Sartor ME, Espinoza F, Quarin CL and Ortiz JPA. 2008. Genetic and embryological evidences of apomixis at the diploid level in Paspalum rufum support recurrent auto-polyploidization in the species. Sexual Plant Reproduction 21:205-215 
Soltis DE and Soltis PS. 2009. The role of hybridization in plant speciation. Annual Review of Plant Biology 6o:561-588

Stebbins GL. 1950. Variation and Evolution in Plants. Columbia University Press, New York

Stitt $M$ and Hurry V. 2002. A plant for all seasons: alterations in photosynthetic carbon metabolism during cold acclimation in Arabidopsis. Current Opinion in Plant Biology 5(3):199-206

Takuno S and Gaut BS. 2013. Gene body methylation is conserved between plant orthologs and is of evolutionary consequence. Proceedings of the National Academy of Sciences of the United States of America. 110(5):1797-1802

Talent $\mathbf{N}$ and Dickinson TA. 2007. Endosperm formation in aposporous Crataegus (Rosaceae, Spiraeoideae, tribe Pyraceae): parallels to Ranunculaceae and Poaceae. New Phytologist 173:231-249

Theodoridis S, Randin C, Broennimann O, Patsiou T and Conti E. 2013. Divergent and narrower climatic niches char- acterize polyploid species of European primroses in Primula sect, Aleuritia. Journal of Biogeography 40:1278-1289

Vinkenoog R, Bushell C, Spielman M, Adams S, Dickinson HG and Scott RJ. 2003. Genomic imprinting and endosperm development in flowering plants. Molecular Biotechnology 25:149-184

Voigt ML, Melzer M, Rutten T, Mitchell-Olds T and Sharbel TF. 2007. Gametogenesis in the apomictic Boechera holboellii complex: the male perspective. In: Hörandl E, Grossniklaus U, Van Dijk PJ, Sharbel T (eds) Apomixis: evolution, mechanisms and perspective. Gantner, Ruggell, pp 235-258

Vrijenhoek RC and Parker ED Jr. 2009. Geographical Parthenogenesis: General Purpose Genotypes and Frozen Niche Variation. In: Schön I, Martens K and Van Dijk PJ (eds) Lost Sex. Springer, Dordrecht, pp 99-131

Wang J, Tian L, Madlung A, Lee H-S, Chen M, Lee JJ, Watson B, Kagochi T, Comai L and Chen ZJ. 2004. Stochastic and epigenetic changes of gene expression in Arabidopsis polyploids. Genetics 167:1961-1973

Zhang J, Liua Y, Xiaa E-H, Yaoa Q-Y, Liud X-D and Gaoa L-Z. 2015. Autotetraploid rice methylome analysis reveals methylation variation of transposable elements and their effects on gene expression. Proceedings of the National Academy of Sciences of the United States of America. 112(50):7022-7029 


\section{Conclusion}

The provided findings indicate a natural and random emergence of tetraploid individuals from several facultative diploid mother plants via a triploid bridge. Polyploidy as result of whole genome duplication (autotetraploidy) increased their physiological tolerance, especially to the harsher conditions at higher altitudes, establishing the respective populations in colder climates. A combination of endogenous selection for apomictic seed formation and exogenous selection for cold-adapted polyploid genotypes resulted in the predominance of apomictic polyploids in higher altitudes of the Alps, where diploids have no fitness benefit.

Observed predominance of apomixis among tetraploids may facilitated colonization and rapid range expansion over the higher elevated areas of the central to eastern Alps, without restrictions in mating partners and pollinators, while diploids failed to expand their occurrences after the last glaciation.

Multiple origins of tetraploid apomicts and the regular intermixing due to high degrees of facultative sexuality preserved essential epigenetic structures. Extreme cold conditions at the niche margins then altered methylation patterns and hence differentiated facultative and obligate tetraploid apomicts of $R$. kuepferi. 


\section{Thesis Declarations}

Chapter 1 - Correlations of polyploidy and apomixis with elevation and associated environmental gradients in an alpine plant

CCFS, SD, SK and EH designed and conceived the research. CCFS, BK AD, MW, SD and EH collected plant materials. CCFS and SK performed the laboratory work. CCFS and BK analyzed data. CCFS is the main author of this article and wrote the manuscript with contributions of all co-authors.

Chapter 2 - Pathways to polyploidy: indications of a female triploid bridge in the alpine species Ranunculus kuepferi (Ranunculaceae)

CCFS and EH designed and conceived the research. CCFS, BK and EH collected plant materials. CCFS performed the laboratory work and analyzed data. CCFS is the main author of this article and wrote the manuscript with contributions of all co-authors.

Chapter 3 - Epigenetic patterns and Geographical Parthenogenesis in the alpine species Ranunculus kuepferi (Ranunculaceae)

CCFS, SD and EH designed and conceived the research. CCFS, BK, SK, SD and EH collected plant materials. CCFS and ES performed the laboratory work and CCFS analyzed data. CCFS is the main author of this article and wrote the manuscript with contributions of all co-authors. 


\section{List of Publications}

(in order of appearance)

Schinkel CCF, Kirchheimer B, Dullinger S, Klatt S, Syngelaki E and Hörandl E. 2018. Epigenetic patterns and Geographical Parthenogenesis in the alpine species Ranunculus kuepferi (Ranunculaceae). Manuscript Draft.

Kirchheimer B, Wessely J, Gattringer G, Hülber K, Moser D, Schinkel CCF, Appelhans M., Klatt S, Caccianiga M, Dellinger A, Guisan A, Kuttner M, Lenoir J, Maiorano L, Nieto-Lugilde D, Plutzar C, Svenning JC, Willner W, Hörandl E and Dullinger S. 2018. Reconstructing Geographical Parthenogenesis: effects of niche differentiation and reproductive mode on Holocene range expansion of an alpine plant. Ecology Letters 21:392-401.

Klatt S, Schinkel CCF, Kirchheimer B, Dullinger S and Hörandl E. 2018. Effects of cold treatments on fitness and mode of reproduction in the diploid and polyploid alpine plant Ranunculus kuepferi (Ranunculaceae). Annals of Botany 121(7):1287-1298.

Schinkel CCF, Kirchheimer B, Dullinger S, Geelen D, De Storme N and Hörandl E. 2017. Pathways to polyploidy: indications of a female triploid bridge in the alpine species Ranunculus kuepferi (Ranunculaceae). Plant Systematics and Evolution 303(8):10931108.

Schinkel CCF, Kirchheimer B, Dellinger AS, Klatt S, Winkler M, Dullinger S and Hörandl E. 2016. Correlations of polyploidy and apomixis with elevation and associated environmental gradients in an alpine plant. AoB PLANTS 8:1-16.

Kirchheimer B, Schinkel CCF, Dellinger AS, Klatt S, Moser D, Winkler M, Lenoir J, Caccianiga M, Guisan A, Nieto-Lugilde D, Svenning JC, Wilfried Thuiller W, Vittoz P, Willner W, Zimmermann NE, Hörandl E and Dullinger S. 2016. A matter of scale: Apparent niche differentiation of diploid and tetraploid plants may depend on extent and grain of analysis. Journal of Biogeography 43:716-726. 


\section{Curriculum Vitae}

04/2013-02/2019 Doctor Thesis

University of Goettingen, Germany

DFG-funded project on evolutionary implication of asexuality in a new established alpine model plant (Ranunculus kuepferi), in collaboration with the University of Vienna

04/2013-05/2017 Researcher University of Goettingen, Germany Autonomous research activity; Planning and execution of experiments; Management of financial and laboratory resources; Supervision of students and assistants

10/2009-12/2011 Master of Science Biology

University of Gießen, Germany "Influence of the reproductive mechanism on the different molecular evolution of nuclear and chloroplast markers"

$10 / 2006-10 / 2009$

Bachelor of Science Biology

University of Gießen, Germany "Investigations on the host-parasite system Phrag-midium - Rosa rugosa"

2002-2005 Abitur

Justus-Liebig-Gymnasium, Gießen, Germany

Advanced courses - Biology and Informatics 